\title{
Inter-string Bose-Einstein Correlations in Hadronic Z Decays using the L3 Detector at LEP
}





\title{
Inter-string Bose-Einstein Correlations in Hadronic $\mathrm{Z}$ Decays using the L3 Detector at LEP
}

\author{
Een wetenschappelijke proeve op het gebied van de \\ Natuurwetenschappen, Wiskunde en Informatica
}

Proefschrift

\begin{abstract}
ter verkrijging van de graad van doctor aan de Radboud Universiteit Nijmegen

op gezag van de rector magnificus prof. mr. S.C.J.J. Kortmann, volgens besluit van het College van Decanen in het openbaar te verdedigen op donderdag 4 september 2008

om 13.30 uur precies
\end{abstract}

door

\section{Qin Wang}

geboren op 26 januari 1979

te Nanzhang,

China 
Promotor:

Copromotor:

Manuscriptcommissie:
Prof. dr. E.W. Kittel

Dr. W.J. Metzger

Prof. dr. G. Gustafson, Lund University

Prof. dr. N. de Groot

Prof. dr. T. Csörgő, MTA KFKI RMKI, Budapest and University of São Paulo 


\section{To Aleh \& Nastya}

for your love, patience, support and understanding... 



\section{Contents}

1 Introduction $\quad 1$

1.1 Bose-Einstein Correlations . . . . . . . . . . . . . . . . . . 1

1.2 String Model . . . . . . . . . . . . . . . . . . 5

1.3 Motivation of Inter-string BEC Study _ . . . . . . . . . . . . . . . . 7

1.4 Analysis Methods . . . . . . . . . . . . . . . . . 8

2 Experimental Setup $\quad 11$

2.1 The LEP Collider . . . . . . . . . . . . . . . . . . . . . . . . . . 11

2.2 The L3 Detector . . . . . . . . . . . . . . . . . . . . . . . . . . . . . 14

2.2.1 The Silicon Microvertex Detector . . . . . . . . . . . . 15

2.2.2 The Time Expansion Chamber and Z Chamber . . . . . . . . . . . . . 16

2.2.3 The Electromagnetic Calorimeter . . . . . . . . . . . . 18

2.2 .4 The Hadron Calorimeter . . . . . . . . . . . . . . . . . . 18

2.3 Trigger and Data Acquisition . . . . . . . . . . . . . . . . . . . 19

2.4 Event Reconstruction . . . . . . . . . . . . . . . . . . . . . . . . . 19

2.5 Detector Simulation . . . . . . . . . . . . . . . . . 20

3 Event and Track Selection $\quad 21$

3.1 Calorimeter Based Event Selection . . . . . . . . . . . . . . . . . . . 21

3.2 TEC Based Track Selection . . . . . . . . . . . . . . . . . . . . . 25

3.3 TEC Inefficiencies $\ldots \ldots \ldots \ldots \ldots$

3.4 Further Event Selection Based on Tracks . . . . . . . . . . . . . . . . . . 32

3.5 Light-Quark and b-Quark Event Selection . . . . . . . . . . . . . . . . . 33

3.6 Jet Clustering Algorithm . . . . . . . . . . . . . . . . . . . . . . . 37

3.7 Selection of Gluon Jets and Quark Jets ～. . . . . . . . . . . . . . . . . 38

3.8 Special 3-jet Topologies: Mercedes, Yq, and Yg Events . . . . . . . . . . . . . 40

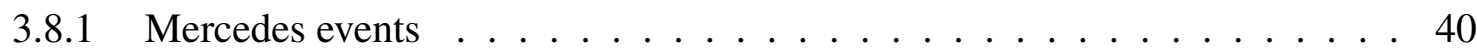

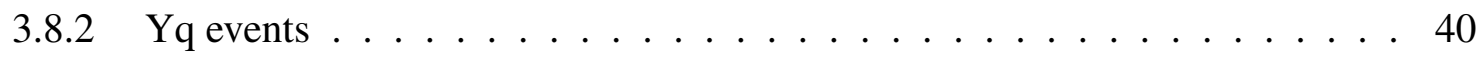

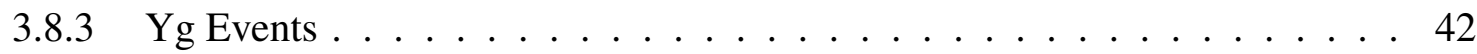


4 Monte Carlo Generators $\quad 45$

4.1 Hadron Production in $\mathrm{e}^{+} \mathrm{e}^{-}$Collisions . . . . . . . . . . . . . . . . . 45

4.2 Four Monte Carlo Generators . . . . . . . . . . . . . . . . . . . . . . . 47

4.3 Bose-Einstein Correlations in Monte Carlo Generators . . . . . . . . . . . . . . 47

4.4 Tuning of Monte Carlo Parameters . . . . . . . . . . . . . . . . . . . . . 48

5 Comparison of BEC in 2-jet and 3-jet Events 49

5.1 Mixed Reference Sample . . . . . . . . . . . . . . . . . . . . . . . 49

5.2 Detector Correction . . . . . . . . . . . . . . . . . 50

5.3 Dependence of $R_{2}$ on Number of Jets and Multiplicity . . . . . . . . . . . 55

5.3 .1 Comparison of $\lambda$ and $R$ in 2- and 3-jet Events . . . . . . . . . . 55

5.3.2 Multiplicity Dependence of $\lambda$ and $R$ in 2- and 3-jet Events . . . . . . . . 55

5.4 Conclusion . . . . . . . . . . . . . . . . . 60

6 Comparison of Quark and Gluon Jets 63

6.1 Hardness Dependence of $\lambda$ and $R$ in Quark and Gluon Jets . . . . . . . . . . 63

6.2 Comparison of Quark and Gluon Jets with Similar Energy . . . . . . . . . . . . 69

6.2 .1 Quark and Gluon Jets in Mercedes Events . . . . . . . . . . . . . . . . 69

6.2.2 Quark and Gluon Jets in Yq events . . . . . . . . . . . . . . . 70

6.2.3 Gluon Jet in the Yg Events and Quark Jet in the 2-jet Events . . . . . . . . 70

6.3 Rapidity and $x$ Dependence of $\lambda$ and $R$ in Quark and Gluon Jets . . . . . . . 72

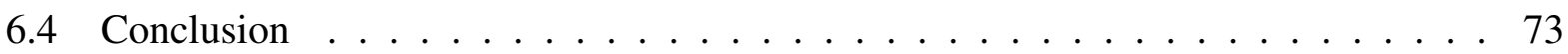

7 Comparison of Same Side and Different Sides of Gluon Jet 77

7.1 Feasibility of Studying Same Side and Different Sides of a Gluon Jet . . . . . . . 78

7.1.1 Check of the Separation of Gluon and Quark Jets at Generator Level . . . . 78

7.1.2 Check of the Deviation of the Jet Direction from the Gluon Direction . . . 79

7.1.3 Feasibility of Using $R_{2}$ to Compare the Same Side and Different Sides of the Gluon Jet . . . . . . . . . . . . . . . . . . . . . 80

7.2 Comparison of the Same Side and Different Sides of the Gluon Jet . . . . . . . . 81

7.2.1 Gluon Jets in All 3-jet Events . . . . . . . . . . . . . . 85

7.2.2 Gluon Jets in Yq events . . . . . . . . . . . . . . . 85

7.2.3 Gluon Jets in Mercedes Events . . . . . . . . . . . . . . . . . . . . . 87

7.2 .4 Gluon Jets in Yg Events . . . . . . . . . . . . . . . . . 88

7.3 Rapidity and $x$ Dependence of the Differences . . . . . . . . . . . . . . 89

7.3.1 Gluon Jets in All 3-jet Events . . . . . . . . . . . . . . . . . 90

7.3.2 Gluon Jets in Yq events . . . . . . . . . . . . . . . . 90

7.3.3 Gluon Jets in Mercedes Events . . . . . . . . . . . . . . . . 90 
7.3.4 Gluon Jets in Yg Events . . . . . . . . . . . . . . . . . 90

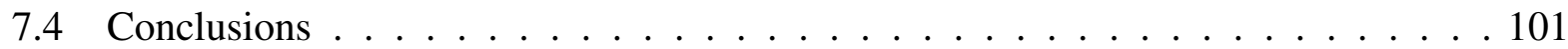

8 Conclusion $\quad 103$

$\begin{array}{ll}\text { References } & 105\end{array}$

$\begin{array}{lr}\text { Summary } & 109\end{array}$

$\begin{array}{ll}\text { Samenvatting } & 111\end{array}$

$\begin{array}{lr}\text { Acknowledgements } & 113\end{array}$

$\begin{array}{ll}\text { Curriculum Vitae } & 115\end{array}$ 



\section{Chapter 1}

\section{Introduction}

In this thesis, Bose-Einstein Correlations (BEC) are studied using data from the L3 experiment at the Large Electron Positron collider (LEP) of CERN. Here, an introduction is given to BoseEinstein Correlations, the string model, and our motivation to study inter-string BEC. Furthermore, the general analysis method for this thesis is presented.

\subsection{Bose-Einstein Correlations}

Pauli showed in 1940 [1] that particles with arbitrary half-integer spin (fermions) obey Fermi-Dirac statistics, while particles with arbitrary integer spin (bosons) follow Bose-Einstein (BE) statistics. This indicates that bosons tend to occupy the same state, while fermions must occupy different states.

In 1957, Hanbury Brown and Twiss (HBT) invented and used intensity interferometry for the measurement of stellar radii [2]. With this method, one detects average products of intensity rather than average products of amplitude (fields). The particle physics equivalent of the Hanbury-BrownTwiss effect is the Goldhaber-Goldhaber-Lee-Pais (GGLP) [3] effect. In 1959, in a bubble chamber study of charged pion production in proton-antiproton collisions at $1.05 \mathrm{GeV}$, the observation was made [4] that the angular distribution of like-charged pion pairs was different from that of unlike-charged pairs. In 1960, GGLP [3] interpreted this observation by introducing Bose-Einstein statistics for the two identical pions, though the relationship of this interpretation with the HBT experiment had not yet been realized at that time.

It has been pointed out $[5,6]$ that not only can the study of pion correlations reveal the spacetime structure of the pion production region, but that it also can provide information on the degree of coherence of the produced pion field. Thus BEC could ideally provide both geometrical and dynamical information on particle production in a given reaction.

Generally, the Bose-Einstein correlation function between two identical bosons is defined as

$$
R_{2}=\frac{\rho_{2}\left(p_{1}, p_{2}\right)}{\rho_{0}\left(p_{1}, p_{2}\right)}
$$

where $\rho_{2}\left(p_{1}, p_{2}\right)$ is the two-particle probability density of two identical bosons with four-momenta 


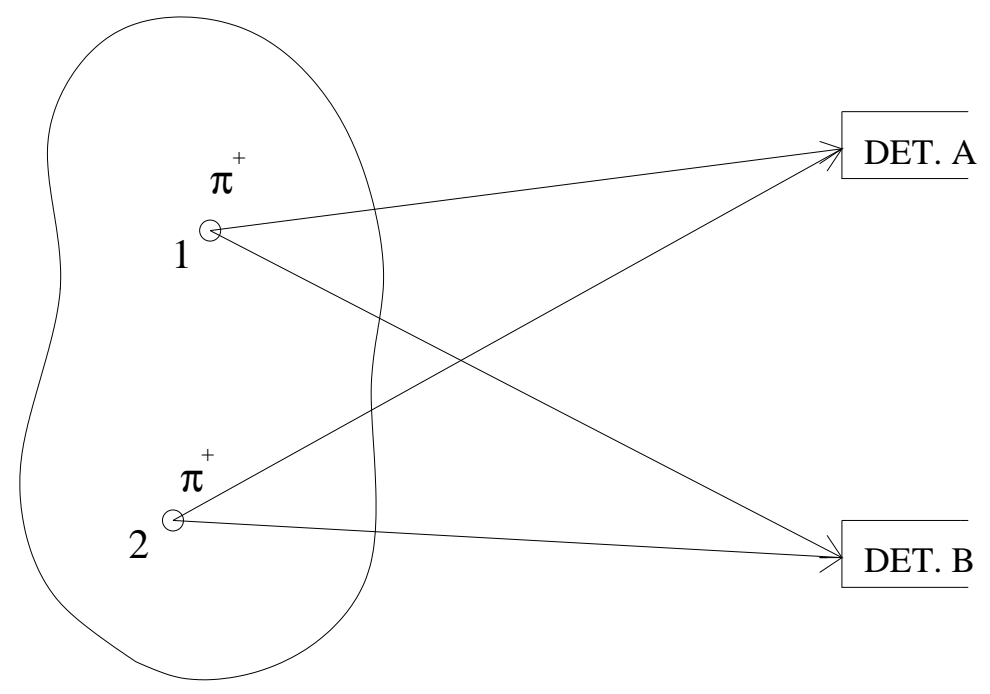

Figure 1.1: Two identical pions emitted from space-time points $x_{1}$ and $x_{2}$ are detected by two detectors at $x_{\mathrm{A}}$ and $x_{\mathrm{B}}$. The trajectories of the two pions are not distinguishable.

$p_{1}$ and $p_{2}$ in the experimental data sample, and $\rho_{0}\left(p_{1}, p_{2}\right)$ is the same density in a reference sample which contains all correlations except BEC.

The usual derivation of the BEC function uses the wave function approach [7]. Let us first consider a source which consists of a number of discrete emission points $i$, each of which is characterized by a probability amplitude of emission

$$
F\left(x_{i}\right)=\int \mathrm{d}^{4} x F(x) \delta\left(x-x_{i}\right),
$$

where $F(x)$ is the probability amplitude distribution.

Let $\psi(x, p)$ be the wave function of a particle emitted at a space-time position $x$ with 4momentum $p$. The total probability $\rho(p)$ of observing the emission of a particle with momentum $p$ from the source is obtained by summing the contributions of all points $i$. This summation can be done coherently (summation of amplitudes) or incoherently (summation of probabilities) depending on whether the phases of the discrete amplitudes $F\left(x_{i}\right)$ are coherent or incoherent. In the first case we get the coherent single-particle probability

$$
\rho_{\mathrm{C}}(p)=\left|\sum_{i} F\left(x_{i}\right) \psi\left(x_{i}, p\right)\right|^{2}
$$

and in the second case the incoherent single-particle probability

$$
\rho_{\mathrm{I}}(p)=\sum_{i}\left|F\left(x_{i}\right) \psi\left(x_{i}, p\right)\right|^{2} .
$$

Similarly, let $\psi\left(x_{i}, x_{j}, p_{1}, p_{2}\right)$ be the wave function of two particles emitted with 4-momentum $p_{1}$ and $p_{2}$ at any combination of two emission points $\left(x_{i}, x_{j}\right)$. The probability of observing two particles with momenta $p_{1}$ and $p_{2}$ for coherent emission is 


$$
\begin{aligned}
\rho_{\mathrm{C}}\left(p_{1}, p_{2}\right)= & \frac{1}{2}\left|\sum_{i, j} \psi\left(x_{i}, x_{j}, p_{1}, p_{2}\right) F\left(x_{i}\right) F\left(x_{j}\right)\right|^{2} \\
= & \frac{1}{2} \sum_{i, j, k, l} \psi\left(x_{i}, x_{j}, p_{1}, p_{2}\right) F\left(x_{i}\right) F\left(x_{j}\right) \psi_{p_{1}, p_{2}}^{*}\left(x_{k}, x_{l}\right) F\left(x_{k}\right)^{*} F\left(x_{l}\right)^{*} \\
= & \frac{1}{2} \sum_{i, j}\left|\psi\left(x_{i}, x_{j}, p_{1}, p_{2}\right)\right|^{2}\left|F\left(x_{i}\right)\right|^{2}\left|F\left(x_{j}\right)\right|^{2} \\
& +\frac{1}{2} \sum_{i, j} \psi\left(x_{i}, x_{j}, p_{1}, p_{2}\right) \psi^{*}\left(x_{j}, x_{i}, p_{1}, p_{2}\right)\left|F\left(x_{i}\right)\right|^{2}\left|F\left(x_{j}\right)\right|^{2} \\
& +\frac{1}{2} \sum_{i, j \neq k, l} \psi\left(x_{i}, x_{j}, p_{1}, p_{2}\right) \psi^{*}\left(x_{k}, x_{l}, p_{1}, p_{2}\right) F\left(x_{i}\right) F\left(x_{j}\right) F\left(x_{k}\right)^{*} F\left(x_{l}\right)^{*} .
\end{aligned}
$$

For the incoherent case, the corresponding two-particle probability is

$$
\rho_{\mathrm{I}}\left(p_{1}, p_{2}\right)=\sum_{i, j}\left|\psi\left(x_{i}, x_{j}, p_{1}, p_{2}\right)\right|^{2}\left|F\left(x_{i}\right)\right|^{2}\left|F\left(x_{j}\right)\right|^{2}
$$

For a symmetric wave function we recognize that the sum of the first two terms in Eq. (1.5) is just the right-hand side of Eq. (1.6). So we get

$$
\rho_{\mathrm{C}}\left(p_{1}, p_{2}\right)=\rho_{\mathrm{I}}\left(p_{1}, p_{2}\right)+\frac{1}{2} \sum_{i, j \neq k, l} \psi\left(x_{i}, x_{j}, p_{1}, p_{2}\right) \psi^{*}\left(x_{k}, x_{l}, p_{1}, p_{2}\right) F\left(x_{i}\right) F\left(x_{j}\right) F\left(x_{k}\right)^{*} F_{l}^{*} .
$$

If the bosons originate from a chaotic source, the second term on the right-hand side of Eq. (1.7) fluctuates randomly and drops out in the expectation value $\left\langle\rho_{\mathrm{C}}\left(p_{1}, p_{2}\right)\right\rangle$. Thus a chaotic source is effectively incoherent.

Instead of a discrete emission source, we consider now a source whose emission is continuously distributed in space-time. Then we obtain the analog of Eq. (1.6):

$$
\rho_{\mathrm{I}}\left(p_{1}, p_{2}\right)=\int\left|\psi\left(x_{1}, x_{2}, p_{1}, p_{2}\right)\right|^{2} f\left(x_{1}\right) f\left(x_{2}\right) \mathrm{d}^{4} x_{1} \mathrm{~d}^{4} x_{2},
$$

where $f(x)=|F(x)|^{2}$ is the density distribution of the source.

If the wave function $\psi\left(x_{1}, x_{2}, p_{1}, p_{2}\right)$ for observing two identical bosons produced at points $x_{1}$ and $x_{2}$ with four-momenta $p_{1}$ and $p_{2}$ in detectors located at $x_{\mathrm{A}}$ and $x_{\mathrm{B}}$ (see Fig. 1.1) is simplified as a plane wave function, we obtain

$$
\psi\left(x_{1}, x_{2}, p_{1}, p_{2}\right)=\frac{1}{\sqrt{2}}\left[\mathrm{e}^{i p_{1}\left(x_{\mathrm{A}}-x_{1}\right)} \mathrm{e}^{i p_{2}\left(x_{\mathrm{B}}-x_{2}\right)}+\mathrm{e}^{i p_{1}\left(x_{\mathrm{A}}-x_{2}\right)} \mathrm{e}^{i p_{2}\left(x_{\mathrm{B}}-x_{1}\right)}\right],
$$

where the second term in the bracket comes from the symmetrization required by BE statistics. The reason is that the two bosons are not distinguishable and we cannot tell from which point $\left(x_{1}\right.$ or $x_{2}$ ) the boson is emitted.

We obtain the probability of observing these two bosons by squaring the wave function:

$$
\left|\psi\left(x_{1}, x_{2}, p_{1}, p_{2}\right)\right|^{2}=1+\frac{1}{2} \mathrm{e}^{-i\left(p_{1}-p_{2}\right)\left(x_{1}-x_{2}\right)}+\frac{1}{2} \mathrm{e}^{i\left(p_{1}-p_{2}\right)\left(x_{1}-x_{2}\right)}=1+\cos (Q \cdot \Delta x)
$$


where $Q=p_{1}-p_{2}$ is the four-momentum difference and $\Delta x=x_{1}-x_{2}$ is the space-time difference.

Assume that the source points $x_{1}$ and $x_{2}$ are randomly distributed in a region of space specified by a normalized density distribution $f(x)$. Then the incoherent two-particle probability density is

$$
\begin{aligned}
\rho_{2}\left(p_{1}, p_{2}\right) & =\iint \mathrm{d}^{4} x_{1} \mathrm{~d}^{4} x_{2}\left|\psi\left(x_{1}, x_{2}, p_{1}, p_{2}\right)\right|^{2} f\left(x_{1}\right) f\left(x_{2}\right) \\
& =\iint \mathrm{d}^{4} x_{1} \mathrm{~d}^{4} x_{2}(1+\cos (Q \cdot \Delta x)) f\left(x_{1}\right) f\left(x_{2}\right) .
\end{aligned}
$$

If the two particles in a reference sample were distinguishable, i.e., BE symmetry were not needed, then the second term of (1.9) would not be present and the probability density of the reference sample would be

$$
\rho_{0}=\iint \mathrm{d}^{4} x_{1} \mathrm{~d}^{4} x_{2} f\left(x_{1}\right) f\left(x_{2}\right)=1
$$

Thus, the BEC function (1.1) in the case of completely incoherent production can be written as

$$
R_{2}\left(p_{1}, p_{2}\right)=1+\iint \mathrm{d}^{4} x_{1} \mathrm{~d}^{4} x_{2} \cos (Q \cdot \Delta x) f\left(x_{1}\right) f\left(x_{2}\right)=1+|G(Q)|^{2},
$$

where $G(Q)$ is the Fourier transform of the source density distribution $f(x)$,

$$
G(Q)=\int \mathrm{d}^{4} x \mathrm{e}^{i Q \cdot x} f(x)
$$

The second term in Eq. (1.13) is the consequence of the Bose-Einstein correlation between the two parts of wave function (1.9). We expect $R_{2}-1$ from Eq. (1.13) to reflect the absolute square of the Fourier transform of the space-time distribution of the source.

The simplest assumption for the space-time density distribution in the rest frame of the source is the static, symmetric Gaussian

$$
f(x)=\frac{1}{4 \pi^{2} R^{4}} \mathrm{e}^{-\left(\vec{x}^{2}\right) / 2 R^{2}} .
$$

Note that the term static here means a lack of time dependence defined by Eq. (1.15) corresponding to source elements being at rest. Substituting this Gaussian in (1.14) yields

$$
R_{2}\left(p_{1}, p_{2}\right)=1+\mathrm{e}^{-Q^{2} R^{2}} \text {. }
$$

From Eq. (1.16) we see that $R_{2} \rightarrow 2$ for $Q \rightarrow 0$, assuming totally incoherent emission. On the other hand, totally coherent emission results in $R_{2}=1$. An extra parameter $\lambda$, which can be interpreted as the degree of incoherence, is introduced [8]:

$$
R_{2}\left(p_{1}, p_{2}\right)=1+\lambda \mathrm{e}^{-Q^{2} R^{2}}
$$

where $\lambda=0$ for the totally coherent case, and $\lambda=1$ for the totally incoherent case.

We need to note that the assumption is made in the above derivation that the bosons are free during propagation. In fact, the Coulomb effect can reduce the value of $R_{2}$ at small $Q$. Fortunately, the influence is small for $\mathrm{e}^{+} \mathrm{e}^{-}$collisions and is only observed in the first bin of $Q$ [9]. Therefore, Coulomb effects are neglected in this analysis.

Other final-state interactions, such as strong interactions between the two pions [10-12] are expected to be negligible [10-12] and are usually not considered. 


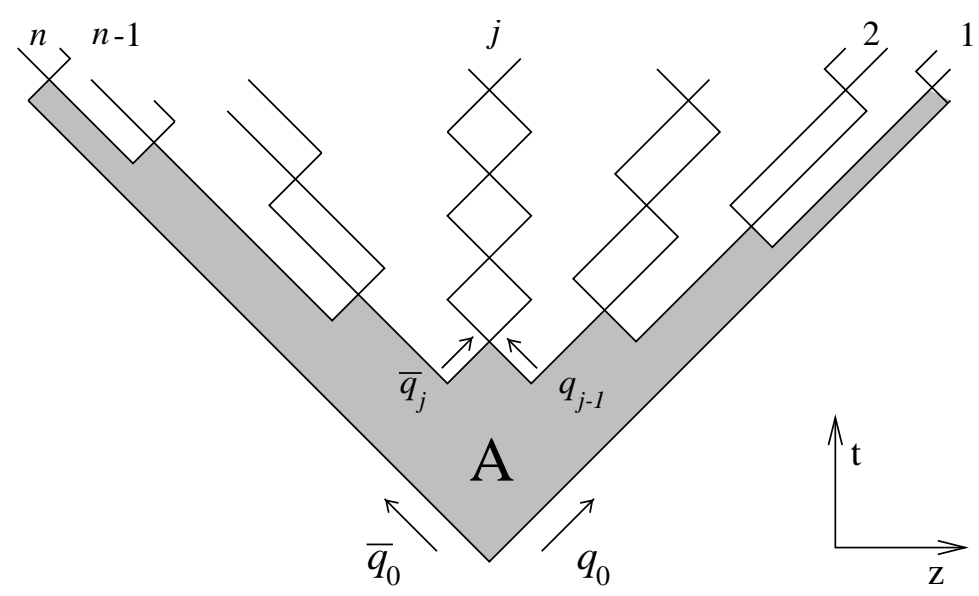

Figure 1.2: Illustration of the decay of a Lund string into a final state $(1,2, \ldots, n)$.

\subsection{String Model}

Quantum Chromodynamics is a theory of the strong interaction. However, accurate calculations are only possible for hard processes. This works well to obtain many useful formulae at parton level, but it does not quantitatively describe how quarks and gluons combine to form the colorless hadrons observed in experiment. This hadronization process happens at a low energy scale where the strong coupling constant $\alpha_{\mathrm{s}}$ is large. To describe hadronization, phenomenological models are used. Among these models, a cluster model [13] and a string model [14] are quite successful. In this section, I describe briefly the string model, especially how it predicts Bose-Einstein correlations on the basis of coherent production. The cluster model is described in Chapter 4, where the different mechanisms of various Monte Carlo generators are discussed.

In $\mathrm{e}^{+} \mathrm{e}^{-}$annihilation, the primary quark $\mathrm{q}$ and anti-quark $\overline{\mathrm{q}}$ separate from each other with high energy, thus stretching a color field. In the string model, the color field is approximated by a massless relativistic string. The endpoints of the string are identified with quark and anti-quark properties, while an emitted gluon appears as a "kink" or transverse excitation of the string. The string can break up into two pieces, a new quark or anti-quark appearing at each new stringend. This process continues and the final-state mesons are formed from a quark and an anti-quark originating from adjacent vertices, as illustrated in Fig. 1.2

The basic input of the string model is the Lund area law [15]. As we can see from Fig. 1.2, there is an area $A$ spanned by the string before the break-up into mesons. The production probability of a set of hadrons is related to this area $A$.

Firstly, if there is a final state containing $n$ identical bosons, there are $n$ ! ways to produce such a state, each corresponding to a different permutation of the particles. For each permutation, $\mathscr{P}$, of particles, the production matrix element (as in Fermi's Golden Rule) is expressed as:

$$
\mathscr{M}_{\mathscr{P}}=\exp (i \kappa-b / 2) A_{\mathscr{P}}
$$

where $\kappa$ is the string tension (phenomenologically $\kappa \approx 1 \mathrm{GeV} / \mathrm{fm}$ ) and $b$ the correlation length in rapidity. According to quantum mechanics, the transition matrix element is to be symmetrized with 


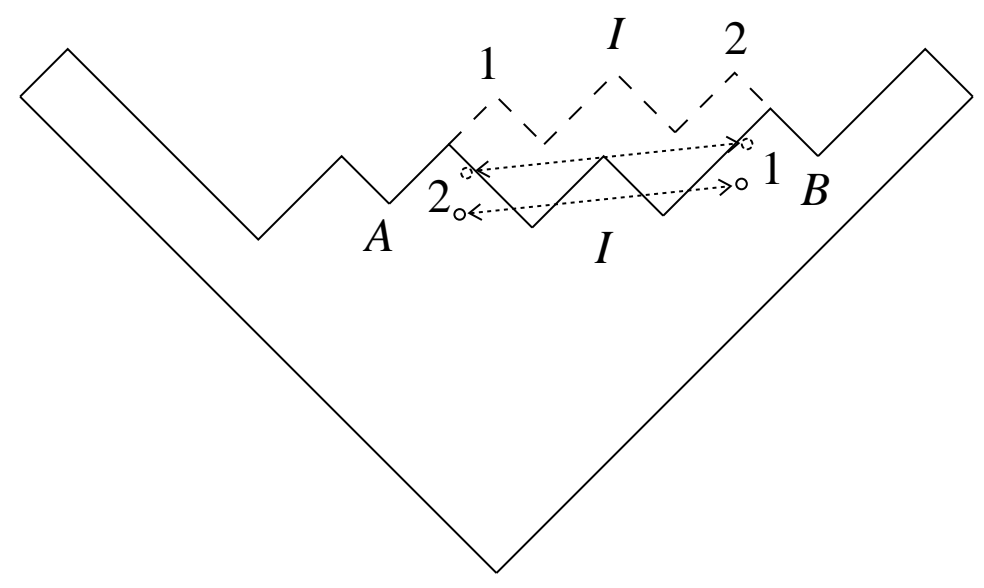

Figure 1.3: Illustration of two possible ways of producing a final state which contains $n$ identical particles.

respect to exchange of identical bosons. This leads to the general expression for the production amplitude,

$$
\mathscr{M}=\sum_{\mathscr{P}} \mathscr{M}_{\mathscr{P}}
$$

where the sum runs over all possible permutations of the identical bosons. The cross section then contains the square of the symmetrized amplitude $\mathscr{M}$

$$
|\mathscr{M}|^{2}=\sum_{\mathscr{P}}\left(\left|\mathscr{M}_{\mathscr{P}}\right|^{2}\left(1+\sum_{\mathscr{P}^{\prime} \neq \mathscr{P}} \frac{2 \operatorname{Re}\left(\mathscr{M}_{\mathscr{P}} \mathscr{M}_{\mathscr{P}^{\prime}}{ }^{*}\right)}{\left|\mathscr{M}_{\mathscr{P}}\right|^{2}+\left|\mathscr{M}_{\mathscr{P}}\right|^{2}}\right)\right)
$$

The second term reflects the interference between different ways to produce identical bosons. In a Monte Carlo program it can be introduced by weighting the produced event with a factor

$$
\omega=1+\sum_{\mathscr{P}^{\prime} \neq \mathscr{P}} \frac{2 \operatorname{Re}\left(\mathscr{M}_{\mathscr{P}} \mathscr{M}_{\mathscr{P}{ }^{\prime}}{ }^{*}\right)}{\left|\mathscr{M}_{\mathscr{P}}\right|^{2}+\left|\mathscr{M}_{\mathscr{P}}\right|^{2}}
$$

The outer sum in Eq. (1.20) is taken care of by generating many events.

Let us examine two different ways of producing this final state (Fig. 1.3). Two identical bosons are denoted $(1,2)$ and the state between them is denoted by $I$. By exchanging bosons 1 and 2 , the two permutations $(\ldots, 1, I, 2, \ldots)$ and $(\ldots, 2, I, 1, \ldots)$ reflect the same final state, but the two production configurations, in general, correspond to different areas. Therefore, the area difference $\Delta A=\left|A_{12}-A_{21}\right|$ appears and enters into the weight while calculating the production probability. The weight, in terms of the area difference due to the interchange of the two bosons and after introduction of a transverse momentum $k_{\perp}$ term, then reads 


$$
\omega=1+\sum_{\mathscr{P}^{\prime} \neq \mathscr{P}} \frac{\cos \frac{\Delta A}{2 \kappa}}{\cosh \left(\frac{b \Delta A}{2}+\frac{\Delta\left(\sum k_{\perp j}^{2}\right)}{2 \sigma_{k_{\perp}}^{2}}\right)},
$$

where $\Delta$ denotes the difference between the configurations $\mathscr{P}$ and $\mathscr{P}^{\prime}$, and the sum of $k_{\perp j}^{2}$ runs over all vertices. $\sigma_{k_{\perp}}$ is the width of the transverse momentum distribution for the generated hadrons.

Finally, the two-particle correlation function $R_{2}$ can be obtained by dividing the two-particle probability density of bosons with and without weights,

$$
R_{2}\left(p_{1}, p_{2}\right)=\frac{\rho_{2 \omega}\left(p_{1}, p_{2}\right)}{\rho_{2}\left(p_{1}, p_{2}\right)} \approx 1+\cos (\kappa \Delta A) / \cosh (b \Delta A / 2)
$$

In the limit of $Q=0$, one has $\Delta A=0$ and $R_{2}=2$. Thus the coherent string model predicts an enhancement of $R_{2}(0)=2$, just like the conventional HBT effect. However, the HBT approach assumed incoherent production, whereas the string model adds the amplitudes coherently.

\subsection{Motivation of Inter-string BEC Study}

It was repeatedly emphasized by the Lund group [15-19] that in a string model, Bose-Einstein correlations among particles produced from a single string only depend on local properties of the string and thus should be independent of the surrounding environment in which the string fragments. The BEC function can be derived through the Lund area law for each string separately, as described in Sect. 1.2. Hence, no BEC are predicted for particles produced by different strings.

Inter-string BEC have been widely studied in the process $\mathrm{e}^{+} \mathrm{e}^{-} \rightarrow \mathrm{W}^{+} \mathrm{W}^{-} \rightarrow$ hadrons at LEP because of their possible influence on the measurement of the $\mathrm{W}$ mass. Present combined results from LEP give no statistically significant evidence for inter-string BEC between the two W's [20]. However, the low statistics of WW events limits the possibility of detecting it.

Another source of two-string events is $Z$ decay to three jets, i.e., $\mathrm{e}^{+} \mathrm{e}^{-} \rightarrow \mathrm{q} \overline{\mathrm{q}} \mathrm{g} \rightarrow$ three jets, where one string is spanned between the radiated gluon and the primary quark and one between the gluon and the anti-quark (Fig. 1.4). This process has the advantage of much higher statistics than the $\mathrm{W}^{+} \mathrm{W}^{-}$process, and of a smaller average distance expected between the two strings than in WW events.

The correlation strength $\lambda$ is expected $[21,22]$ to decrease when the number of independent overlapping sources increases. The source radius $R$ is expected to increase when the two sources are dependent, since color flux runs from one string over the gluon tip to the other string, so that the distance between two points on the two strings is along this longer curve rather than along a straight line. Thus we expect:

(1) If there are no inter-string BEC but the two strings overlap:

$$
\lambda_{2 \text {-string }}<\lambda_{1 \text {-string }} \quad R_{2 \text {-string }} \approx R_{1 \text {-string }}
$$

(2) If there are inter-string BEC and overlap between the two strings:

$$
\lambda_{2 \text {-string }} \approx \lambda_{1 \text {-string }} \quad R_{2 \text {-string }} \gtrsim R_{1 \text {-string }}
$$




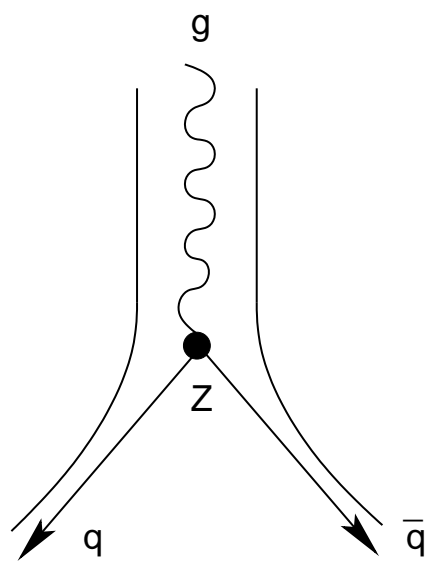

Figure 1.4: String picture of three-jet event.

(3) If there are no BEC between two non-overlapping sources:

$$
\lambda_{2} \approx \lambda_{1} \quad R_{2} \approx R_{1}
$$

(4) If there are BEC between two non-overlapping sources:

$$
\lambda_{2} \approx \lambda_{1} \quad R_{2}>R_{1}
$$

The first preliminary results on 2- and 3-jet events as well as on quark and gluon jets, based on DELPHI data, found no evidence [23] for expectations (1) and (4). Here we shall use L3 data to study 2- and 3-jet events (Chapter 5) and quark and gluon jets (Chapter 6) in more detail and extend the study to a comparison of BEC of pairs of identical pions produced on the same side of the gluon jet to those of identical pions originating from different sides (Chapter 7).

\subsection{Analysis Methods}

In the original definition of the correlation function as in Eq. (1.1), $\rho_{2}$ is a function of the two fourmomenta. However, BEC are large only when $p_{1} \approx p_{2}$, and it has been found that the correlation function can be adequately parametrized as a function of the four-momentum difference $Q$,

$$
Q=\sqrt{-\left(p_{1}-p_{2}\right)^{2}},
$$

which has the clear advantage of reducing $\rho$ from a six-dimensional function to a one-dimensional one. Hence, two-particle BEC are investigated here using the function

$$
R_{2}=\frac{\rho_{2}(Q)}{\rho_{0}(Q)} .
$$

Here, $\rho_{2}(Q)$ is normalized to unity:

$$
\rho_{2}(Q)=\frac{1}{N_{\text {pairs }}} \frac{\mathrm{d} N_{\text {pairs }}}{\mathrm{d} Q},
$$


and $\rho_{0}(Q)$ is the two-particle density of a reference sample which contains all correlations except BEC. The normalization of $\rho$ here differs a bit from the usual case where $\rho$ is normalized to $\langle n(n-1)\rangle$, where $n$ is the number of particles. In fact, these two definitions yield the same $R_{2}$ when the average multiplicities of the data and the reference sample are the same. Otherwise, the multiplicity difference is absorbed in the normalization parameter $\gamma$ introduced in Eq. (1.27) below.

The construction of the reference sample is vital since it does not exist in nature. We use two kinds of reference sample:

1. event mixing, where each particle in a mixed event comes from a different experimental event, and where other correlations which are also removed by the mixing are compensated for by Monte Carlo (see Sect. 5.1 for a description of the procedure);

2. Monte Carlo events generated without BEC by the programs Jetset, PythiA, HeRwig and ARIADNE. These programs are described more fully in Chapt.4.

Formula (1.17) is normally used to parametrize $R_{2}$. However, in many cases (1.17) is found not to provide a good description of the data. There have been many discussions (see e.g. [24]) on alternatives to the Gaussian parametrization. However, many of these require model assumptions. We prefer to take a model-independent approach. Since the Gaussian parametrization provides an approximate description of the data, we expand about the Gaussian using the Edgeworth expansion $[25,26]$. Keeping only the lowest-order non-Gaussian term, we obtain

$$
R_{2}(Q)=\gamma\left(1+\delta Q+\varepsilon Q^{2}\right)\left[1+\lambda \mathrm{e}^{-R^{2} Q^{2}}\left(1+\frac{\zeta}{3 !} H_{3}(R Q)\right)\right],
$$

where $H_{3}(R Q)=(\sqrt{2} R Q)^{3}-3 \sqrt{2} R Q$ is the third-order Hermite polynomial, $\gamma$ is an overall normalization and $\left(1+\delta Q+\varepsilon Q^{2}\right)$ is a term to describe long-range momentum correlations not adequately described in the reference sample. As in the Gaussian parametrization, the parameter $\lambda$ measures the strength of the correlation and $R$ reflects the radius of the source, while the parameter $\zeta$ is the third cumulant moment of the distribution. Parametrization (1.27) can give a good fit to the deviation from the Gaussian.

In this thesis, the Edgeworth expansion Eq. (1.27) is used to parametrize $R_{2}$. In fact, which parametrization is used is not so important, as long as it can give a good fit, since we are interested here only in the relative values of the parameters for different samples. 



\section{Chapter 2}

\section{Experimental Setup}

In this chapter, a short description is given of the Large Electron Positron collider (LEP) at CERN and of the L3 detector including its data acquisition and trigger system. Finally, the detector simulation is described.

\subsection{The LEP Collider}

The LEP collider was located at CERN, the European Organization for Nuclear Research near Geneva on the Swiss-French border (see Fig. 2.1). The LEP ring, with a circumference of 26.7 $\mathrm{km}$, was housed in a long tunnel 50-150 m below the ground. It consisted of 8 curved and 8 straight sections. The four experiments, L3, ALEPH, OPAL and DELPHI were located in four of the straight sections.

Operation of the LEP machine was started in 1989. In the first period of LEP running (19891995, LEP1), beams were accelerated to an energy around $45.6 \mathrm{GeV}$. With a center-of-mass energy approximately corresponding to the mass of the $\mathrm{Z}$ boson, this allowed for precise measurements of many parameters of the electroweak model. The second period of LEP running took place from 1995 to 2000 (LEP2). The main goals of this period were to measure properties of the W boson and to search for new particles, such as the Higgs boson. For that, the center-of-mass energy was gradually increased from an intermediate point at $131 \mathrm{GeV}$ to the $\mathrm{W}$ pair production threshold at $161 \mathrm{GeV}$ and, in the end, to the maximum possible energy of $209 \mathrm{GeV}$. LEP finished its task in November 2000, to make place for the Large Hadron Collider.

The complete LEP collider consisted not just of the large LEP ring. The electrons and positrons were stored and accelerated in a number of smaller rings before being injected into the LEP main ring to collide. A schematic overview of the LEP injection scheme is shown in Fig. 2.2.

The acceleration of the beam is done in several stages. First, the electrons are generated by an electron gun and accelerated to $200 \mathrm{MeV}$ by the LEP Injector Linac (LIL) and shot at a tungsten target where positrons are produced. The electrons and positrons are stored in the Electron Positron Accumulator ring (EPA), after which the bunches are accelerated to $3.5 \mathrm{GeV}$ in the Proton Synchrotron (PS). Finally, the Super Proton Synchrotron (SPS) accelerates them to $22 \mathrm{GeV}$ before injection into the LEP ring. There, the electrons and positrons, travelling in opposite directions, 


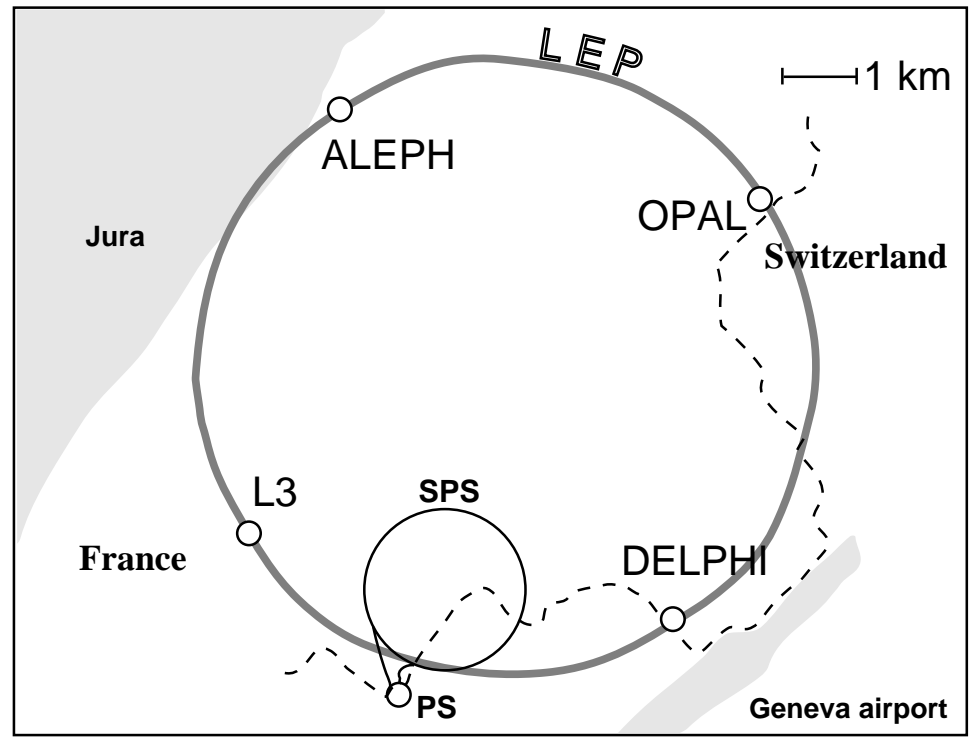

Figure 2.1: Schematic view of the LEP collider.

are accelerated to their final energy and made to collide.

In 1994, when the data used in this thesis were recorded, each LEP beam was composed of four bunches containing on average $3 \times 10^{11}$ particles. The bunches were several centimeters long and a few millimeters high and several millimeter wide over most of the LEP ring. The electrons and positrons were accelerated by radio frequency cavities (RF cavities). These cavities also compensated for the energy loss due to synchrotron radiation. The orbit of the bunches was controlled by 3304 bending magnets. A system of quadrupole and sextupole magnets focused the beams.

The interaction rate at a beam intersection point divided by the interaction cross section is called the luminosity $\mathscr{L}$. It is typically of the order of $10^{31} \mathrm{~cm}^{-2} \mathrm{~s}^{-1}$ and depends on several LEP parameters, such as the beam energy, the number of bunches, the current per bunch, the vertical beam-beam strength parameter, the single-turn frequency, and the focusing strength at the interaction point. The integrated luminosity $\mathscr{L}_{\text {int }}$ is usually expressed in units of $\mathrm{pb}^{-1}\left(1 \mathrm{pb}^{-1}=\right.$ $\left.10^{36} \mathrm{~cm}^{-2}\right)$. It is determined experimentally using small-angle Bhabha scattering $\left(\mathrm{e}^{+} \mathrm{e}^{-} \rightarrow \mathrm{e}^{+} \mathrm{e}^{-}\right)$ via

$$
\mathscr{L}_{\text {int }}=\int_{0}^{T} \mathscr{L} \mathrm{dt}=\frac{N_{\text {Bhabha }}}{\sigma_{\text {Bhabha }}}
$$

where $N_{\text {Bhabha }}$ is the collected number of small-angle Bhabha events in a period of time $T$, and $\sigma_{\text {Bhabha }}$ the Bhabha cross section, which is calculable to high accuracy in Quantum Electrodynamics. Detailed information on the LEP machine and its performance can be found in [27]. Table 2.1 shows an overview of the center-of-mass energies at which LEP operated over years, together with the corresponding integrated luminosity. 


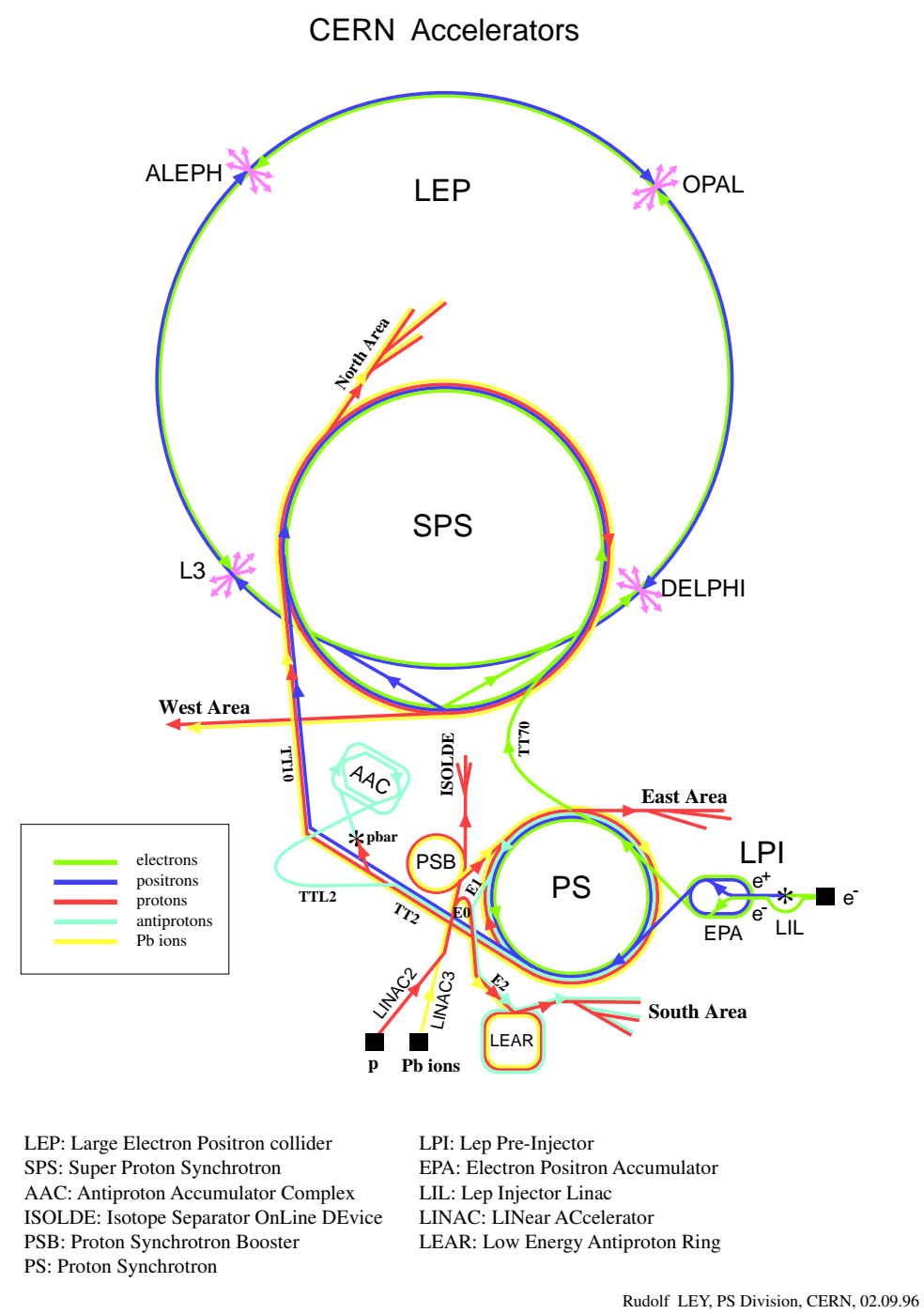

Figure 2.2: Schematic overview of the LEP injection scheme.

\begin{tabular}{|c|c|c|}
\hline year & $\sqrt{s}(\mathrm{GeV})$ & $\mathscr{L}_{\text {int }}\left(\mathrm{pb}^{-1}\right)$ \\
\hline $1989-1995$ & $89-93$ & 155 \\
1995 & $130-136$ & 5 \\
1996 & $161-172$ & 20 \\
1997 & 183 & 55 \\
& $130-136$ & 7 \\
1998 & 189 & 176 \\
1999 & $192-202$ & 230 \\
2000 & $200-209$ & 215 \\
\hline
\end{tabular}

Table 2.1: Center-of-mass energies at which LEP operated and the corresponding integrated luminosity. 


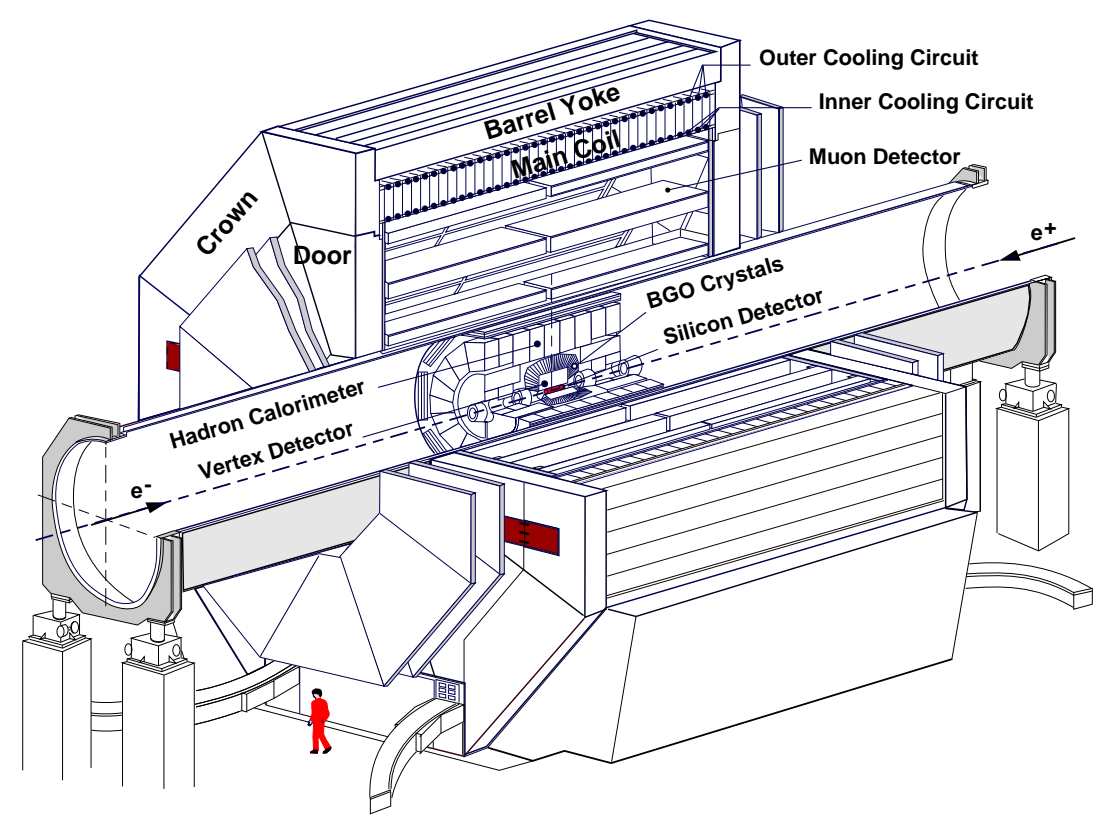

Figure 2.3: Schematic overview of the L3 detector.

\subsection{The L3 Detector}

In this section, the L3 detector is described with emphasis on the central track detector, the most important part of the detector for the analysis reported here.

The design of the L3 detector is optimized for the precise energy measurement of muons, electrons, photons and hadron jets. A perspective view of the detector is shown in Fig. 2.3. The L3 detector was located in a cavern approximately $50 \mathrm{~m}$ underground. The sub-detectors were enclosed in a $16 \mathrm{~m}$ high and $14 \mathrm{~m}$ long octagonally shaped solenoid magnet with a homogeneous magnetic field of $0.5 \mathrm{~T}$ parallel to the beam line. The inner detectors were placed in a $32 \mathrm{~m}$ long and $4.45 \mathrm{~m}$ diameter steel tube within the solenoid.

The L3 detector consisted of various sub-detectors. These sub-detectors were placed cylindrically symmetric along the beam pipe around the interaction point (see Fig. 2.4). From inside out, the main detectors were:

1. the Silicon Microvertex Detector (SMD) to measure accurately the position of a charged particle's trajectory immediately outside the beam pipe;

2. the Central Tracking Chambers consisting of a time expansion chamber (TEC) and the $Z$ chambers to measure the curvature and direction of charged tracks bent by the magnetic field;

3. the Electromagnetic Calorimeter (ECAL) to measure the energy of electrons and photons;

4. the Hadron Calorimeter (HCAL) to measure the energy of hadronic particles; and 


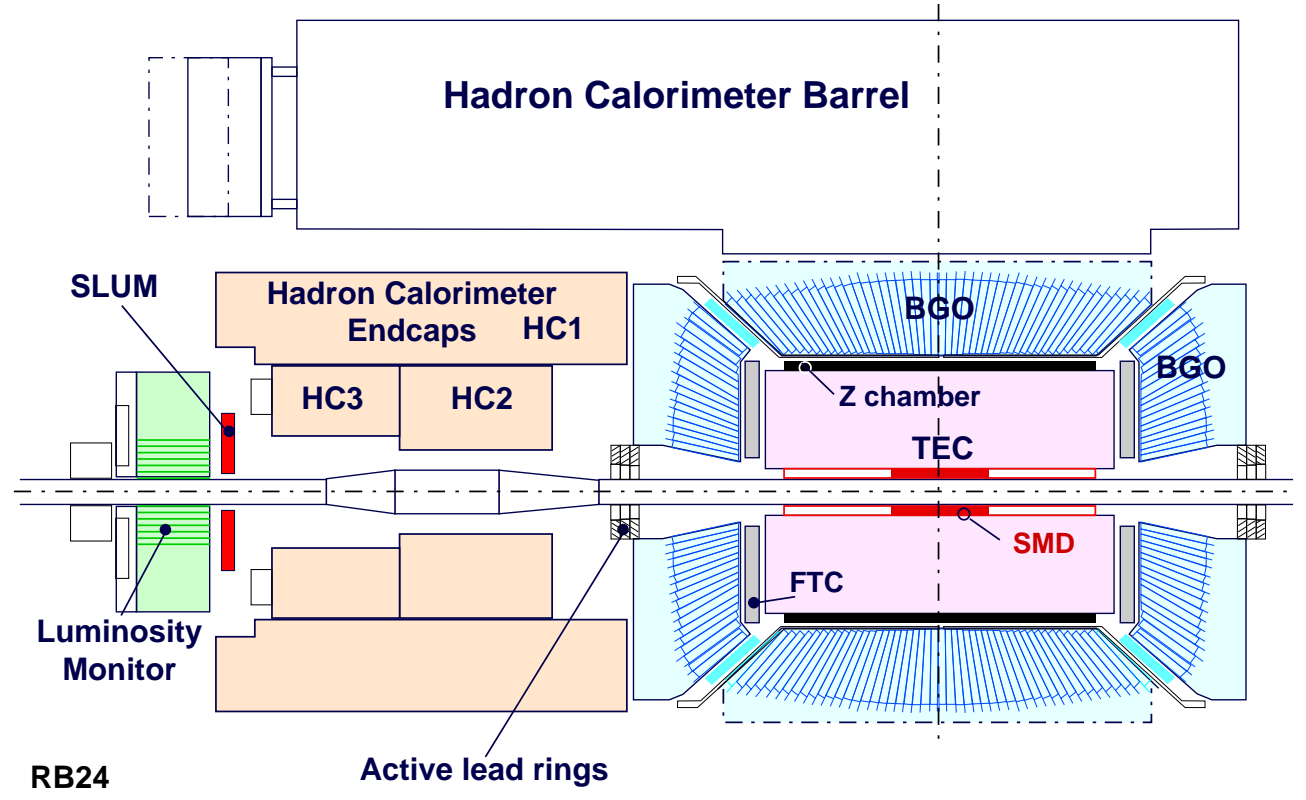

Figure 2.4: A cross section of the L3 detector. Only the left endcap detectors are shown.

5. the Muon Detector (not shown in Fig. 2.4) to measure the momenta of muons.

The coordinate system used is the right-handed L3 coordinate system. The origin is the interaction point of the colliding electron and positron beams. The positive $z$-axis points along the direction of flight of the electron beam, the $x$-axis points towards the center of the LEP ring, and the $y$-axis points vertically upwards. The distance between a point in the $x y$ plane and the interaction point is the radius $r$. The polar angle $\theta \in[0, \pi]$ is the angle between the direction of a particle and the positive direction of the $z$-axis. The azimuthal angle $\phi \in[0,2 \pi]$ is the angle between the radius vector $\vec{r}$ and the positive $x$-axis.

Each of the subdetectors will now be briefly described with exception of the muon detector, which is not used in the analysis of this thesis. A description of the muon detector can be found in $[28,29]$.

\subsubsection{The Silicon Microvertex Detector}

The Silicon Microvertex Detector (SMD) was installed between the beam pipe and the TEC (Fig. 2.4) in 1993 to improve the tracking system and the vertex reconstruction. To accomodate it, the radius of the beam pipe of LEP at the interaction point was reduced from $80 \mathrm{~mm}$ to $55 \mathrm{~mm}$ $[30,31]$. The SMD was used to measure the charged particle trajectory close to the interaction point in order better to resolve possible secondary vertices from the decay of short-lived particles such as hadrons containing $b$ quarks.

The SMD was $30 \mathrm{~cm}$ long and it covered the polar angle range $22^{\circ} \leq \theta \leq 158^{\circ}$. It consisted of two cylindrical layers of double-sided silicon detector ladders, at radius $61.7 \mathrm{~mm}$ and 77.4 mm, respectively (see Fig. 2.5). Each layer consisted of 12 ladders which were made up of two 


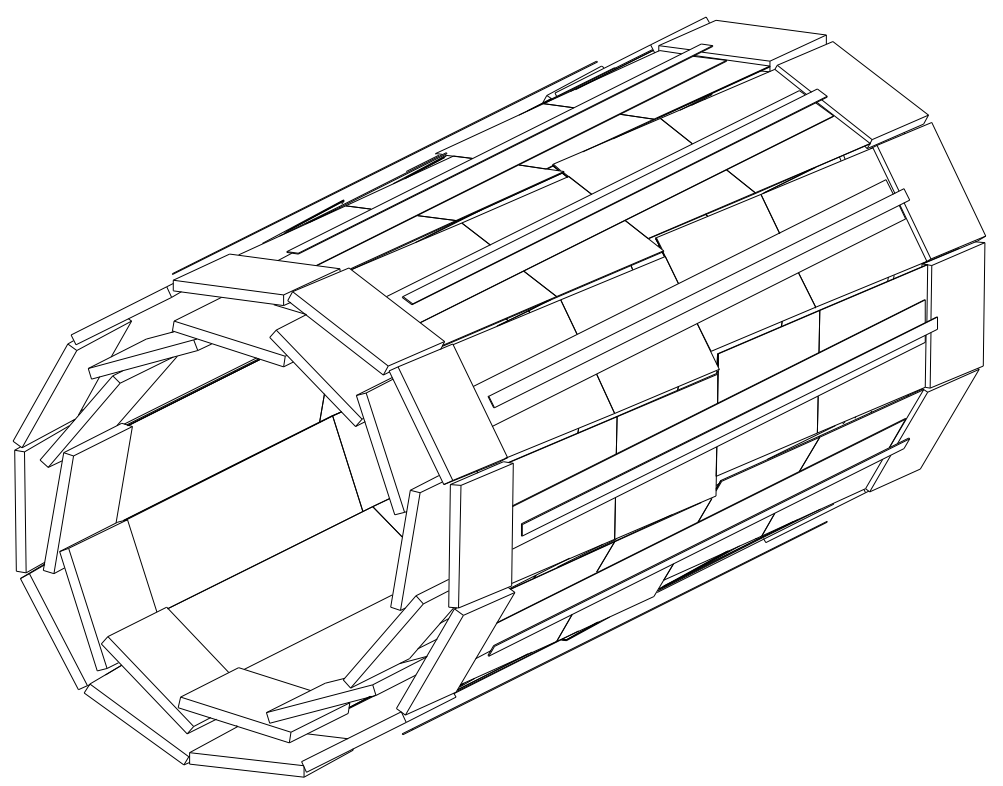

Figure 2.5: Perspective view of SMD ladders.

electrically independent half-ladders. Each half-ladder was made of two $70 \times 40 \mathrm{~mm}^{2}, 300 \mu \mathrm{m}$ thick silicon wafers.

On one side of the silicon wafers, strips were inplanted parallel to the beam line at a $25 \mu \mathrm{m}$ pitch. They provided a high precision $r \phi$ position measurement of a traversing charged particle. There were $36863 r \phi$ channels. On the other side, strips, oriented perpendicular to the $r \phi$ ones with a $50 \mu \mathrm{m}$ pitch gave an $r z$ position. When a charged particle traverses the depleted region of the silicon, it loses energy by ionization causing electron-hole pairs to be created. The electronhole pairs were collected in these strips. The SMD provided high precision 3-dimensional position measurement, close to the interaction region. It could reach a position resolution of $7.5 \mu \mathrm{m}$ in the $r \phi$ direction and $14.3 \mu \mathrm{m}$ in the $z$ direction.

\subsubsection{The Time Expansion Chamber and Z Chamber}

Besides the SMD, the Time Expansion Chamber (TEC) and the Z Chamber provided information for the reconstruction of the trajectory of the charged particles in the $r \phi$ coordinates (TEC) and the $z$ coordinate (Z Chamber). An $x y$ cross section of the SMD, TEC and Z Chamber is shown in Fig. 2.6.

The TEC was a closed gas volume with a gas mixture of $20 \% \mathrm{CO}_{2}$ and $80 \%$ isobutane. Wires, parallel to the $z$ axis, were under high voltage. Charged particles traversing the TEC ionized the gas. In the electric field the freed electrons drifted to the anode wires while the positive ions drifted to the cathode wires. After the electrons passed the drift region, they entered the amplification region where they were accelerated and created an avalanche of ionization, which produced the detectable signals on the anodes. These signals are recorded as "hits". From the arrival time of the drift electrons on the anodes, the distance of the charged particle's trajectory from the anode 

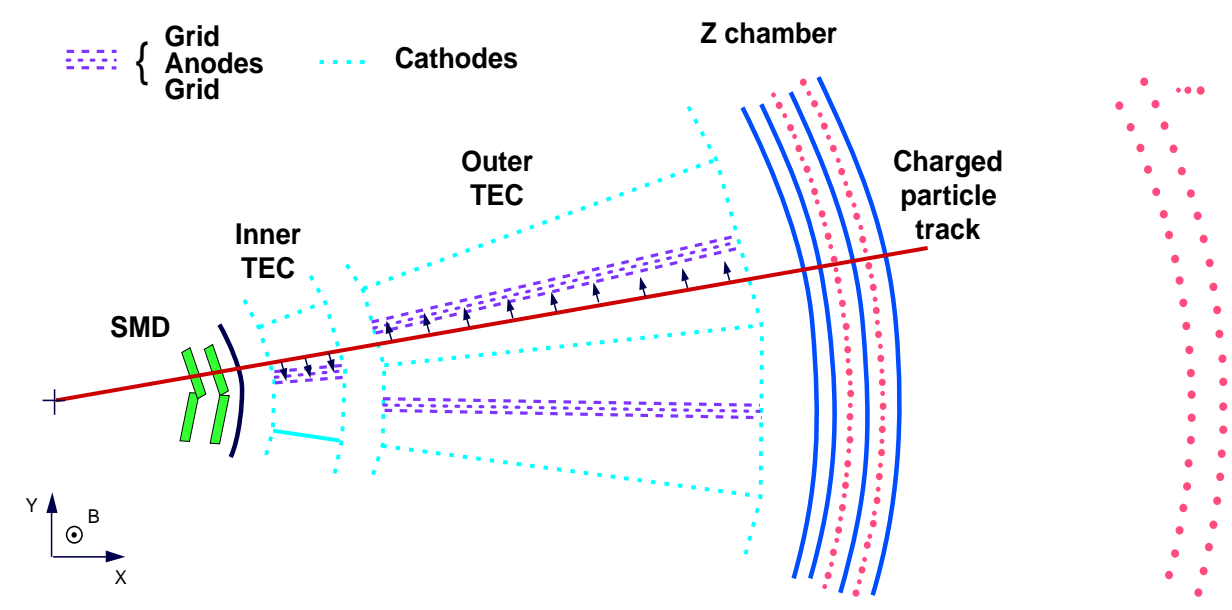

Figure 2.6: An $x y$-view of SMD, TEC and Z Chamber.

can be reconstructed. However, on which side of the anode the particle passed is undetermined, an effect known as left-right ambiguity. The amount of charge detected at the anodes provides a measure of the energy loss of the particle in the TEC.

The TEC consisted of two concentric high precision drift chambers mounted around the beam line: the inner TEC and the outer TEC. The radial distance of the inner TEC was between 8.5 $\mathrm{cm}$ and $14.3 \mathrm{~cm}$ from the beam line, and the outer TEC extended out to $46.9 \mathrm{~cm}$. The inner TEC was subdivided into 12 sectors, each with $30^{\circ}$ coverage in $\phi$ and containing 8 anode wires running parallel to the $z$-axis. The outer TEC was divided into 24 sectors, each with $15^{\circ}$ coverage and containing 54 anode wires, resulting in 62 anode wires in total. This different segmentation of the inner and outer TEC is used to solve the left-right ambiguity. Charge division wires, 2 in the inner TEC and 9 in the outer TEC, were read out on both sides to give approximate $z$-information. The geometry of the TEC was such that only a track with polar angle $44^{\circ} \leq \theta \leq 136^{\circ}$ could reach all 62 wires.

On the outer wall of the TEC, two cylindrical proportional wire chambers ( $Z$ Chamber) with cathode strip readout and anode wires were mounted. A track with a polar angle between $42^{\circ}$ and $138^{\circ}$ passed through the $\mathrm{Z}$ Chamber. This detector provided an additional measurement of the $z$ coordinate at $r=50 \mathrm{~cm}$. The two chambers contained two cathode layers each. The cathode layers were made of 240 strips with a pitch of $4.45 \mathrm{~mm}$. The strips of two of the layers were arranged perpendicular to the $z$ direction and the strips of the other 2 layers ran under a stereo angle of $\pm 60^{\circ}$. The gas mixture consisted of $80 \%$ argon, $16 \% \mathrm{CO}_{2}$, and $4 \%$ isobutane. A charged particle passing through the chamber ionized the gas. The resulting electron avalanche, i.e., the signal measured on the individual cathode strips, was used to determine the coordinate. The $\phi$ component of the stereo layer allowed the matching of the cluster with the TEC track. The $z$ coordinate was measured using the $\mathrm{z}$ layers. The resolution varied with the polar angle and was about $0.2 \mathrm{~mm}$ at $\cos \theta=0$ and 1 $\mathrm{mm}$ at $|\cos \theta|=0.74$. 


\subsubsection{The Electromagnetic Calorimeter}

The Electromagnetic Calorimeter (ECAL) measured the energy of electrons and photons. It contained 10752 BGO (Bismuth Germanate Oxide $\mathrm{Bi}_{4} \mathrm{Ge}_{3} \mathrm{O}_{12}$ ) crystals. Above a certain energy, the electrons and positrons lose their energy mainly through electromagnetic interactions with the nuclei. In the dense matter, electrons with an energy of at least one order of magnitude higher than the electron mass lose energy primarily through Bremsstrahlung, while high energy photons interact through electron-positron pair conversion. In this way, the cascade of Bremsstrahlung emission and electron-positron pair creation from an incoming energetic electron or photon creates a shower. Below the critical energy (about $10 \mathrm{MeV}$ for BGO) the energy loss due to ionization starts to dominate over Bremsstrahlung and pair production and the shower dies out. Ionization and excitation of the crystal becomes important at this point. The excited atoms emit scintillating light, which is detected by photodiodes at the end of the crystal.

The BGO crystals in the ECAL had a length of $24 \mathrm{~cm}$ and were shaped as a truncated pyramid with a front area of $2 \times 2 \mathrm{~cm}^{2}$ and the back face varying from $2.6 \times 2.6 \mathrm{~cm}^{2}$ to $2.9 \times 2.9 \mathrm{~cm}^{2}$. They were mounted with their axis pointing slightly away from the interaction region so that $\gamma$ 's could not pass undetected between the crystals. Two photo-diodes were glued to the rear face of each crystal to detect the BGO scintillation light.

The ECAL was divided into two parts: the barrel and the endcap. The barrel had 7680 crystals and its polar angle coverage was $42^{\circ}<\theta<138^{\circ}$. Each endcap consisted of 1536 BGO crystals, and the coverage was $11^{\circ}<\theta<36^{\circ}$ and $144^{\circ}<\theta<169^{\circ}$.

For electron and photon energies greater than $1 \mathrm{GeV}$ the energy resolution was less than $2 \%$, while a resolution of approximately $1 \%$ is reached at energies of $45 \mathrm{GeV}$.

\subsubsection{The Hadron Calorimeter}

The hadron calorimeter (HCAL) is designed to measure the energy of hadrons emerging from the $\mathrm{e}^{+} \mathrm{e}^{-}$collisions. The HCAL consisted of depleted uranium plates (5-10 mm thick) as an absorber, with a short nuclear interaction length of about $11 \mathrm{~cm}$, and multi-wire proportional chambers $(5.6$ mm thick) as sampling medium. Similar to ECAL, the HCAL also consisted of a barrel part, with a polar angle coverage of $35^{\circ}<\theta<145^{\circ}$, and two endcap parts, with coverage of $6^{\circ}<\theta<35^{\circ}$ and $145^{\circ}<\theta<174^{\circ}$. Both the barrel and endcap parts covered the whole azimuthal angle. The barrel HCAL was made of 144 identical modules grouped into 9 rings and 16 modules. The endcap consisted of three parts, an outer ring and two inner rings. Each of the rings contained 12 modules.

The resolution of a hadronic jet depends on the energy and the direction of the jet. It improves with increasing energy and is better in the central part of the detector than in the outer parts. In back-to-back events $\mathrm{e}^{+} \mathrm{e}^{-} \rightarrow \mathrm{Z} \rightarrow \mathrm{q} \overline{\mathrm{q}}$, the relative energy resolution for a quark jet of $45 \mathrm{GeV}$ in the center of the barrel is about $14 \%$. The direction of the jet axis can be measured with a resolution of about $2.5^{\circ}[32]$. 


\subsection{Trigger and Data Acquisition}

The beam crossing rate at the L3 intersection point was $45 \mathrm{kHz}$, but the interesting physics events did not occur at this rate. A trigger system was designed to make a fast decision on whether a genuine $\mathrm{e}^{+} \mathrm{e}^{-}$event took place and whether or not the event should be recorded for further reconstruction. To avoid dead time during the data taking, this task was performed in three steps.

The level-1 trigger was a logical OR of trigger conditions from 5 independent sub-triggers: the calorimetric trigger, the TEC trigger, the muon trigger, the scintillator trigger and the beamgate trigger. If a positive decision was taken, the data were digitized and recorded in buffer memories; otherwise the electronics were cleared. This was done within $22 \mu \mathrm{s}$, the time between two beam crossings. The trigger rate was $15-25 \mathrm{~Hz}$, depending on trigger settings and beam conditions.

The level-2 trigger received additional information not available in time to be processed at level 1. It correlated signals from different sub-detectors to improve the level-1 decision. Events with more than 1 positive level-1 trigger condition were automatically accepted by the level-2 trigger. The purpose of the level-2 trigger was to reject background events accepted by level-1 but originating from cosmic rays, electronic noise, beam-gas or beam-wall interactions, or synchrotron radiation. The average rejection rate was about $20-30 \%$ at this stage, depending on trigger settings and beam conditions.

The level-3 trigger used the complete digitized raw data available for the event. The selection of good events was based on the correlation of the energy deposited in the ECAL and the HCAL, the reconstruction of muon tracks and the reconstruction of the vertex in the TEC chamber. The trigger rate of level-3 was of the order of $5 \mathrm{~Hz}$.

If the level-3 trigger decision was positive, the information from all sub-detectors was collected and built into one event by a FASTBUS-based data acquisition system and written to tape. A run was defined by the number of events that fit on a $200 \mathrm{MB}$ tape (about 4500 events).

\subsection{Event Reconstruction}

The raw data recorded on tape were processed by the L3 off-line reconstruction program, in order to extract the physical quantities relevant for the physics analysis.

Firstly, the program package REL3 was used to combine the various signals coming from one subdetector into primitive objects (such as energy deposits in the ECAL and hits in TEC). Secondly, the subprogram AXL3 was used to process these objects correlating the information from different subdetectors to obtain objects relevant to physics analysis.

Some of the objects created by the reconstruction program are:

1. Track: The hits in TEC and SMD, together with information from the $Z$ chamber are reconstructed to obtain a track using a pattern recognition and fitting algorithm. The track is also required to be matched to a calorimeter object.

2. ASRC (A Smallest Resolvable Cluster): Information from the calorimeters ECAL/HCAL are combined to form an energy cluster. Adjacent electromagnetic and/or hadronic clusters 
are combined into a single calorimetric cluster to form an ASRC. The ASRC's are used for the hadronic event selection.

3. Jet: Information from different subdetectors are combined to obtain energy (particle) flow or to define hadronic jets.

The data are stored in several formats. The data format used for physics analysis, the DVN, contains only high level detector objects such as energy clusters in ECAL and tracks in TEC.

\subsection{Detector Simulation}

The real detector is not perfect. It is not $100 \%$ efficient, there is always noise present and it has finite resolution. To compare the measurements with theory and to study the effects of the detector response on the event reconstruction, the Monte Carlo generator JETSET (see Chapt. 4) is used to simulate $\mathrm{e}^{+} \mathrm{e}^{-}$events (the generator-level MC event sample).

The generator-level Monte Carlo events are passed through the SIL3 simulation program, which simulates the response of the L3 detector by simulating the interactions of the generated particles with the L3 detector material using the GEANT[33] program. GEANT models the decay of unstable particles, the effect of energy loss, multiple scattering, creation of $\mathrm{e}^{+} \mathrm{e}^{-}$pairs and showers in the detector material. The hadronic interaction processes are simulated by a program package called GHEISHA [34].

There are two levels of detector simulation: One is ideal detector MC simulation in which the time independent detector response, such as acceptance and intrinsic resolution, is simulated. The other is real detector MC simulation where time dependent detector effects are included, such as inefficiencies, malfunctioning of the different subdetectors and beam-gas and beam-wall interactions.

The detector-level MC event samples are stored in the DVN format as used for the data. The effect of the detector on measured physical quantities is studied by comparing the quantities at the generator level of MC to those at the detector level. 


\section{Chapter 3}

\section{Event and Track Selection}

The analysis presented in this thesis is based on the data sample collected by the L3 detector during the 1994 LEP running period at a center-of-mass energy of $\sqrt{\mathrm{s}} \approx 91.2 \mathrm{GeV}$. There are about 3.3 million events in total. In order to obtain a pure sample of $\mathrm{e}^{+} \mathrm{e}^{-} \rightarrow \mathrm{Z} \rightarrow \mathrm{q} \overline{\mathrm{q}}(\mathrm{g})$ events, hadronic event selection is needed. Since in our study of BEC we use the Lorentz invariant four-momentum difference $Q$ of two charged particles, high accuracy on the particle momentum, good precision on the reconstruction of two nearby particles and two-particle resolution are needed. Therefore, a precise selection on the charged tracks used in our analysis is required.

In this chapter, the event and track selections are described. Firstly, the energy deposit in the electromagnetic and hadronic calorimeters is used to select the hadronic events. Secondly, good charged particles are selected on the basis of TEC and SMD information. Thirdly, remaining background events $\left(\mathrm{e}^{+} \mathrm{e}^{-} \rightarrow \mathrm{Z} \rightarrow \tau^{+} \tau^{-}\right)$are removed using the information from TEC.

The quantities chosen to determine the goodness of events and tracks are shown in the following subsections. To judge the efficency of the selection criteria, the distributions of these quantities are compared to a Monte Carlo sample generated by JETSET PS with BEC according to the $\mathrm{BE}_{0}$ algorithm (see Chapt. 4) which was passed through the L3 detector simulation program and fully reconstructed.

Light-quark (u, d, s, c) events are used in most of the analysis. Since b-quark events are needed in the selection of pure gluon jets, (anti-)b-tagging is applied to select pure light-quark and bquark events. The b-tagging algorithm and the light-quark and b-quark event selection is described in Sect. 3.5.

\subsection{Calorimeter Based Event Selection}

The selection of hadronic events is based on calorimeter information. The purpose of using calorimeter information is to reject background as much as possible while not influencing the measurement of charged tracks in TEC. There are two kinds of background events: leptonic $\mathrm{Z}$ decay $\left(\mathrm{Z} \rightarrow \mathrm{e}^{+} \mathrm{e}^{-}, \mu^{+} \mu^{-}, \tau^{+} \tau^{-}\right)$and non-resonant background from sources such as two-photon interactions $\left(\mathrm{e}^{+} \mathrm{e}^{-} \rightarrow \mathrm{e}^{+} \mathrm{e}^{-} \mathrm{q} \overline{\mathrm{q}}\right)$, beam-wall (where a beam particle interacts with the beampipe) and beam-gas (where a beam particle interacts with a gas molecule) interactions. 
Firstly, calorimeter clusters having energy smaller than $100 \mathrm{MeV}$ are discarded, in order to remove contamination from electronic noise. After that, events are selected by requiring:

$$
\begin{gathered}
0.5 \leq \frac{E^{\mathrm{cal}}}{\sqrt{\mathrm{s}}} \leq 1.5 ; \\
\frac{E_{\perp}^{\mathrm{cal}}}{E^{\mathrm{cal}}} \leq 0.55 ; \\
\frac{E_{\|}^{\mathrm{cal}}}{E^{\mathrm{cal}}} \leq 0.4 ; \\
N_{\mathrm{cl}} \geq 14 ; \\
\left|\cos \theta_{\mathrm{thr}}^{\mathrm{cal}}\right| \leq 0.743,
\end{gathered}
$$

where $E^{\mathrm{cal}}=\sum_{i} E_{i}$ is the sum of all calorimeter cluster energies in an event, $E_{\|}^{\mathrm{cal}}=\sum_{i} p_{z i}$ is the energy imbalance along the beam direction, $E_{\perp}^{\mathrm{cal}}=\sqrt{\left(\sum_{i} p_{x i}\right)^{2}+\left(\sum_{i} p_{y i}\right)^{2}}$ is the energy imbalance perpendicular to the beam direction, $N_{\mathrm{cl}}$ is the number of calorimeter clusters in the event, and $\theta_{\mathrm{thr}}^{\mathrm{cal}}$ is the polar angle of the thrust axis, where the thrust axis $\vec{t}$ is defined as the axis maximizing the quantity $\sum_{i}\left|\vec{p}_{i} \cdot \vec{t}\right| / \sum_{i}\left|\vec{p}_{i}\right|$, with $\vec{p}_{i}=\left(E_{i} \sin \theta_{i} \cos \phi_{i}, E_{i} \sin \theta_{i} \sin \phi_{i}, E_{i} \cos \theta_{i}\right)$ being the threedimensional components of the energy of cluster $i$, where $\theta_{i}$ and $\phi_{i}$ are the polar and azimuthal angles of cluster $i$ and $E_{i}$ is its energy. The maximum value of this quantity is called the thrust.

\section{Scaled visible energy}

Hadronic $\mathrm{Z}$ events are characterized by a total visible energy $E^{\mathrm{cal}}$ distributed around the center-ofmass energy $\sqrt{s}$. Fig. 3.1 shows the distribution of the scaled visible energy $\frac{E^{\text {cal }}}{\sqrt{s}}$ for data (dots) and JETSET detector level (histogram) after application of cuts (3.2)-(3.5). The small peak for data in the low energy area is due to background events from $\mathrm{e}^{+} \mathrm{e}^{-} \rightarrow \tau^{+} \tau^{-}$and from the twophoton process, which are not included in the JETSET sample. Note that $\mathrm{e}^{+} \mathrm{e}^{-} \rightarrow \mathrm{Z} \rightarrow \mathrm{q} \overline{\mathrm{q}}$ events have a fraction $E^{\mathrm{cal}} / \sqrt{\mathrm{s}}$ close to unity, while background events have a smaller visible energy. For the two-photon process, the electron and positron remaining within the beam pipe are not detected. Beam-gas and beam-wall events have inherently a smaller total energy, and $\mathrm{e}^{+} \mathrm{e}^{-} \rightarrow \tau^{+} \tau^{-}\left(\mu^{+} \mu^{-}\right)$ events have a small visible energy because of undetected neutrinos in $\tau$ decays or only minimun ionization of muons in calorimeters. The lower cut discriminates against these processes. The upper cut is applied to remove Bhabha events, which are shifted to the high-energy region by the use of scaling factors which are only applicable for hadronic events. 


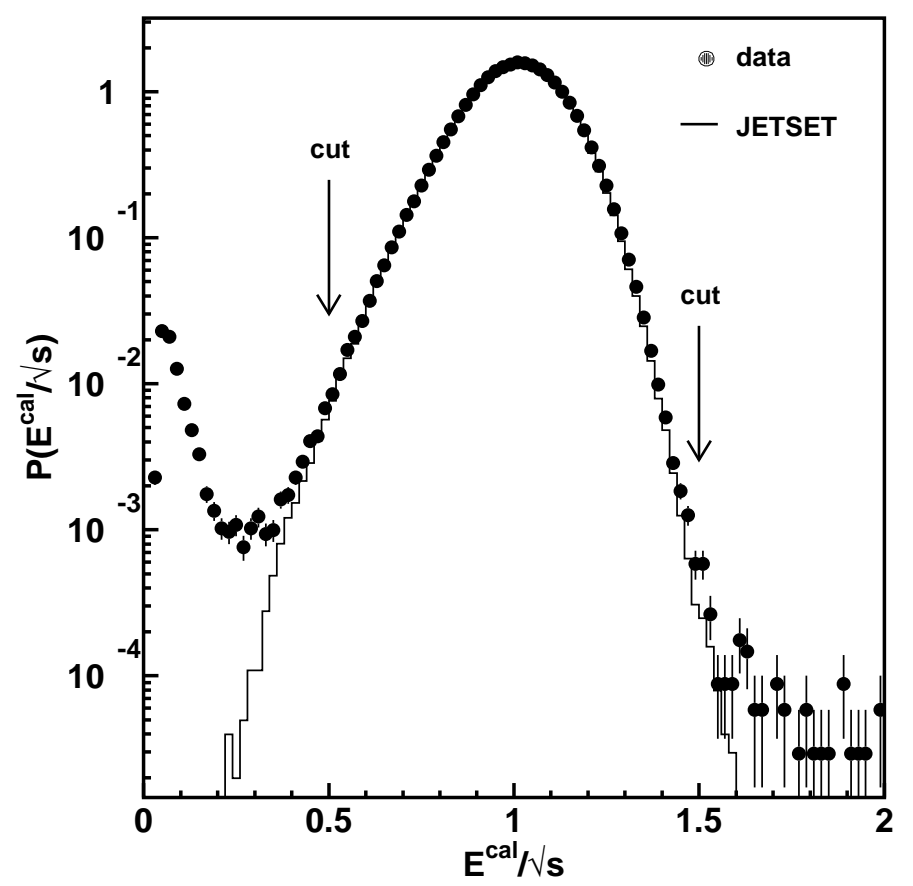

Figure 3.1: Distribution of the total energy of calorimeter clusters normalized by the center-ofmass energy. Data are presented by solid dots and detector-level JETSET by the histogram. Cuts (3.2), (3.3), (3.5) and (3.4) have been applied to both data and MC samples. 


\section{Energy imbalance}

Fig. 3.2 shows the distribution of the energy imbalance along and perpendicular to the beam direction. Since at LEP the laboratory frame is also the center-of-mass frame, the energy flow should be well balanced for hadronic $\mathrm{Z}$ decay events. However, for beam-gas, beam-wall, and two-photon events this is not the case. Eq. (3.2) and Eq. (3.3) are the cuts used to reject these events.
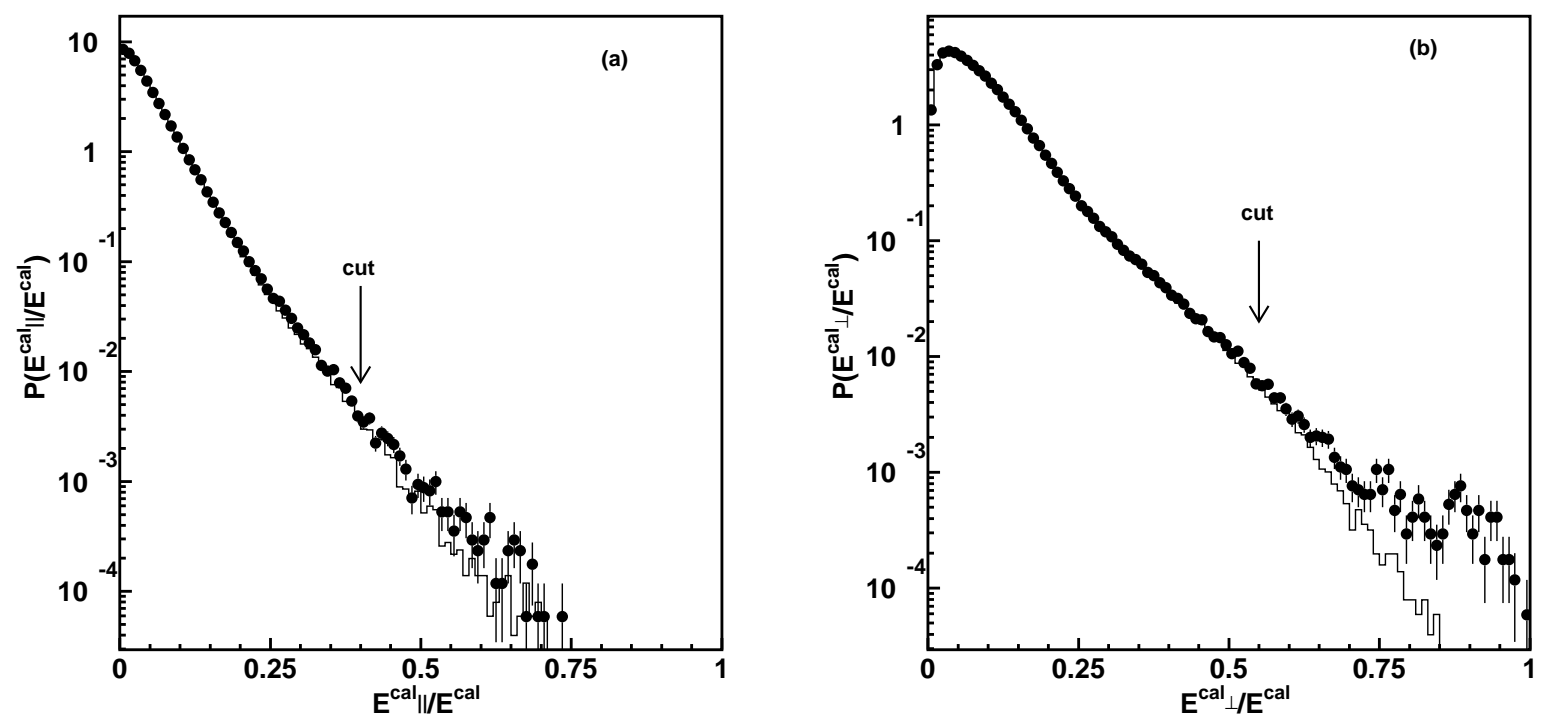

Figure 3.2: (a) Normalized energy imbalance along the beam direction, (b) normalized energy imbalance perpendicular to the beam direction. Data are presented by solid dots and JETSET by the histogram. In addition to the cuts (3.1), (3.5) and (3.4), cut (3.2) has been applied in (a) and cut (3.3) in (b) to both data and MC samples.

\section{Number of ASRC clusters}

Hadronic events normally have a larger particle multiplicity than other processes. Thus a cut rejecting low-multiplicity events reduces the number of background events. By requiring the events to have at least 14 clusters, most $\mathrm{Z} \rightarrow \mathrm{e}^{+} \mathrm{e}^{-}, \mu^{+} \mu^{-}, \tau^{+} \tau^{-}$events, as well as beam-gas and beamwall events are removed.

Fig. 3.3 shows the normalized distribution of the number of clusters. The peak on the left is mainly due to $\tau^{+} \tau^{-}$events. A large disagreement between the data and JETSET is observed in the large-multiplicity region. This is caused by incorrect simulation of hadronic showers in the BGO crystals of the ECAL, and not by background contamination. There is no reason to cut on large cluster multiplicity since it is the charged-particle information from TEC that is used in the analysis.

\section{Theta of thrust axis}

Since the tracks from TEC are used in our analysis, events are chosen which lie within the barrel region of the calorimeter, $35^{\circ}<\theta<145^{\circ}$. This is achieved by requiring the thrust axis to have a 


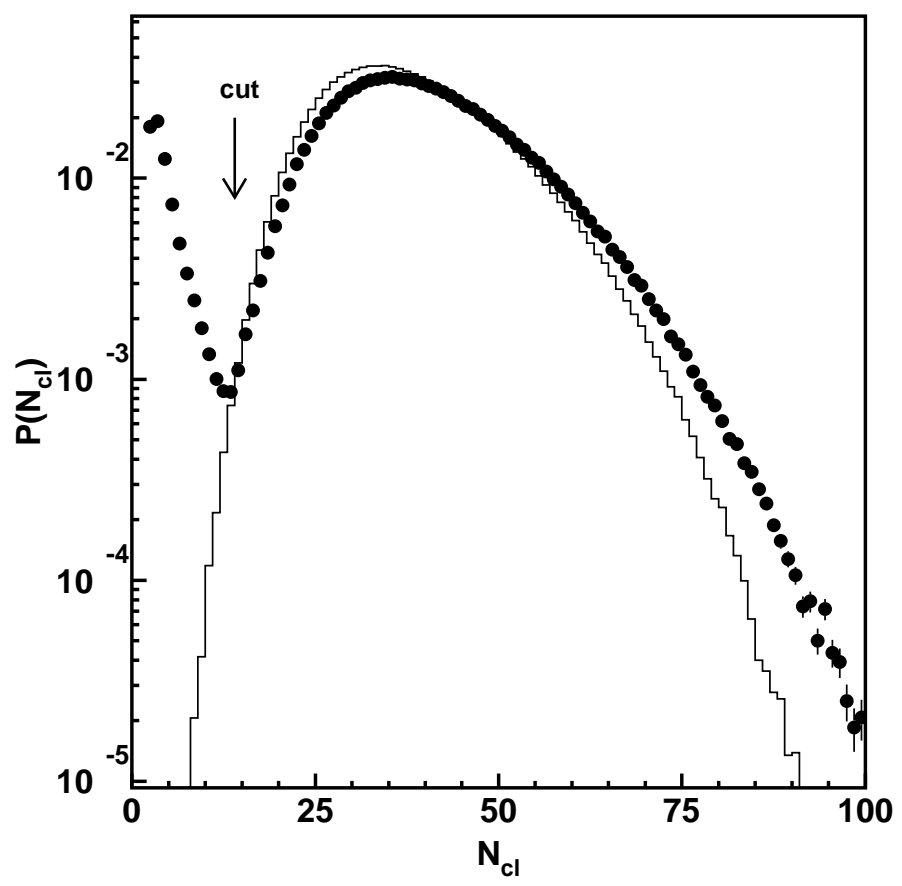

Figure 3.3: Normalized distribution of number of ASRC clusters. Data are presented by solid dots and JETSET by the histogram. Cuts (3.1) to (3.5) have been applied to both data and MC samples.

polar angle satisfying Eq. (3.5) (see Fig. 3.4).

After the above event selection, there are 849,716 events left of the initial 3,373,366 events.

\subsection{TEC Based Track Selection}

After the event selection, we select well measured tracks on the basis of information from the TEC and the SMD by requiring:

$$
\begin{gathered}
|\mathrm{DCA}| \leq 5 \mathrm{~mm} ; \\
\text { inner hits } \geq 1 ; \\
\text { hits } \geq 25 ; \\
\text { span } \geq 40 ; \\
p_{\mathrm{t}} \geq 150 \mathrm{MeV},
\end{gathered}
$$

and by rejecting tracks with 


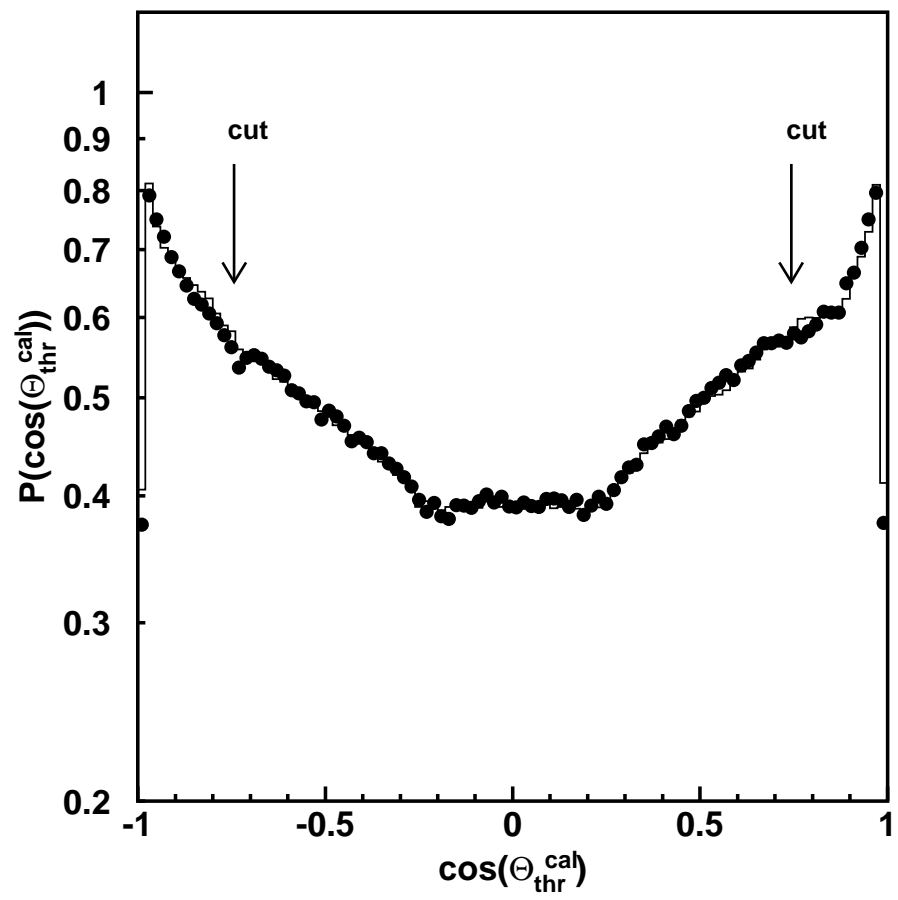

Figure 3.4: Normalized distribution of $\cos \theta_{\mathrm{thr}}^{\mathrm{cal}}$. Data are presented by solid dots, and JETSET by the histogram. Cuts (3.1), (3.2), (3.3) and (3.4) have been applied to both data and MC samples.

$$
45^{\circ}<\phi<52.5^{\circ} \text { or } 225^{\circ}<\phi<232.5^{\circ} \text {. }
$$

\section{Distance of closest approach (DCA)}

To check whether a track originates from the interaction vertex, the track is extrapolated back to the vertex. The distance of closest approach (DCA) is defined as the smallest distance between the track and the interaction point in the plane perpendicular to the beam axis. The DCA is required to be less than or equal to $5 \mathrm{~mm}$ (see Fig. 3.5).

\section{Number of hits}

A track originating at the interaction point passes through the TEC and causes signals ("hits") on a maximum of 62 wires ( 8 wires in the inner TEC and 54 in the outer TEC).

Tracks are required to have at least one inner hit (Fig. 3.6) in order to solve the left-right ambiguity.

The transverse momentum is calculated from the curvature which is obtained from the path formed by subsequent hits. The larger the number of hits, the better is the resolution of the transverse momentum. Misreconstructed tracks usually have a small number of hits. Fig. 3.7 shows the normalized distribution of the number of TEC hits. A discrepancy between data and MC is 


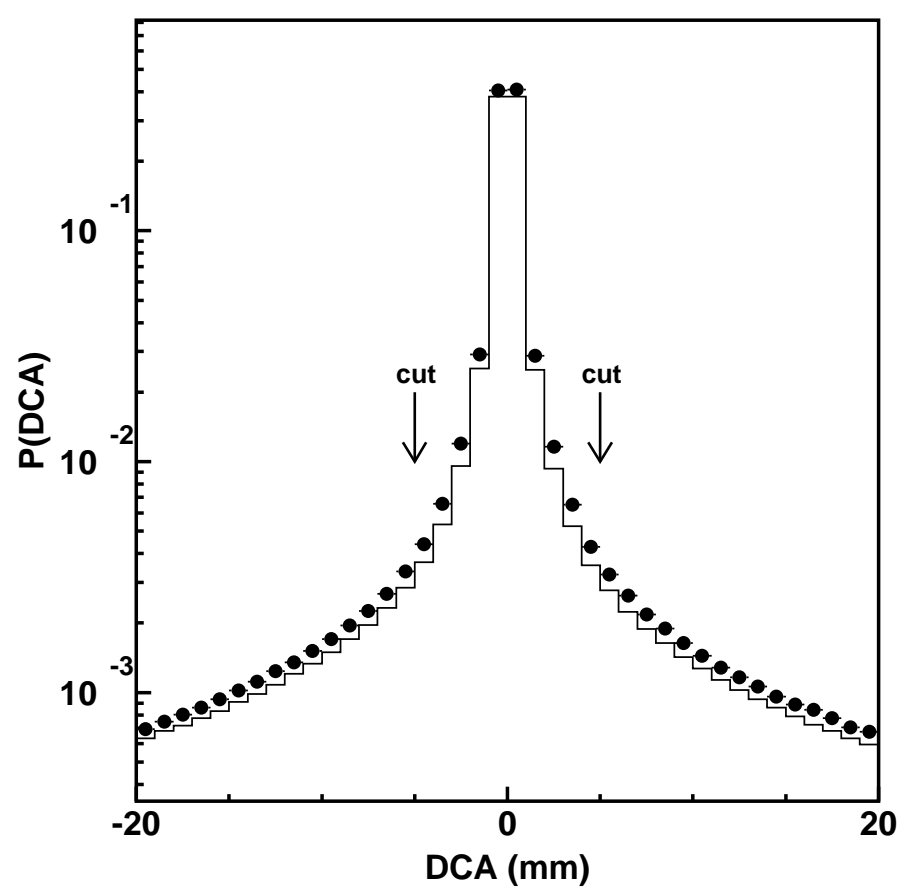

Figure 3.5: Normalized distribution of the distance of closest approach for data (dots) and JETSET (histogram). Cuts (3.7), (3.8), (3.9), (3.10) and (3.11) have been applied.

observed. The cut is chosen to be in the middle of a region where the variation of the disagreement is stable and no big change from bin to bin is expected. A track is required to have at least 25 TEC hits. The loss of track momentum resolution which is caused by the use of such a low requirement on the number of TEC hits is compensated by requiring a large span.

\section{Span of tracks}

The span of a track is defined as the number of wires between the first and the last hit, which is used as a measure of the track length. Misreconstructed tracks generally have a shorter length than well reconstructed tracks. Therefore, we require the track to have a span of at least 40 (Fig. 3.8).

\section{Transverse momentum $p_{\mathrm{t}}$}

Tracks with low transverse momentum experience more multiple scattering, which leads to poor resolution. To avoid such misreconstruction, the transverse momentum is required to be larger than $150 \mathrm{MeV}$ (Fig. 3.9).

\section{Azimuthal angle $\phi$ of tracks}

Due to wrong simulation of the inefficiency of two TEC sectors, large discrepancies between data 


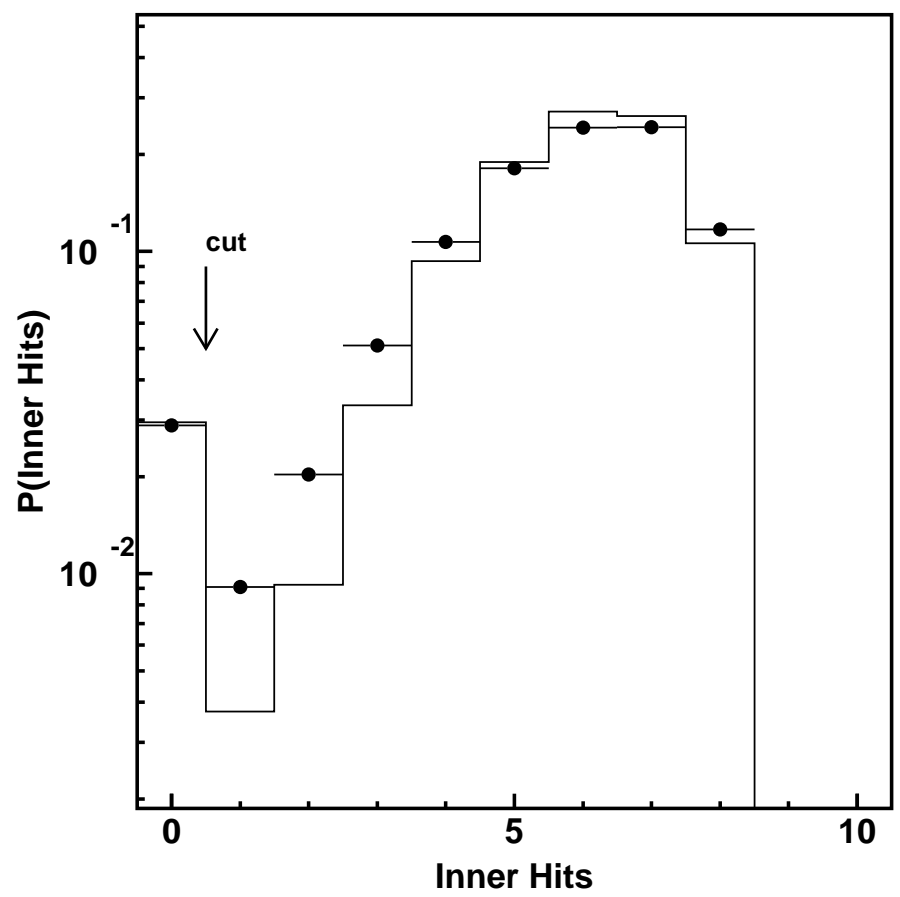

Figure 3.6: Normalized distribution of the number of inner hits for data (dots) and JETSET (histogram). Cuts (3.6), (3.10) and (3.11) have been applied.

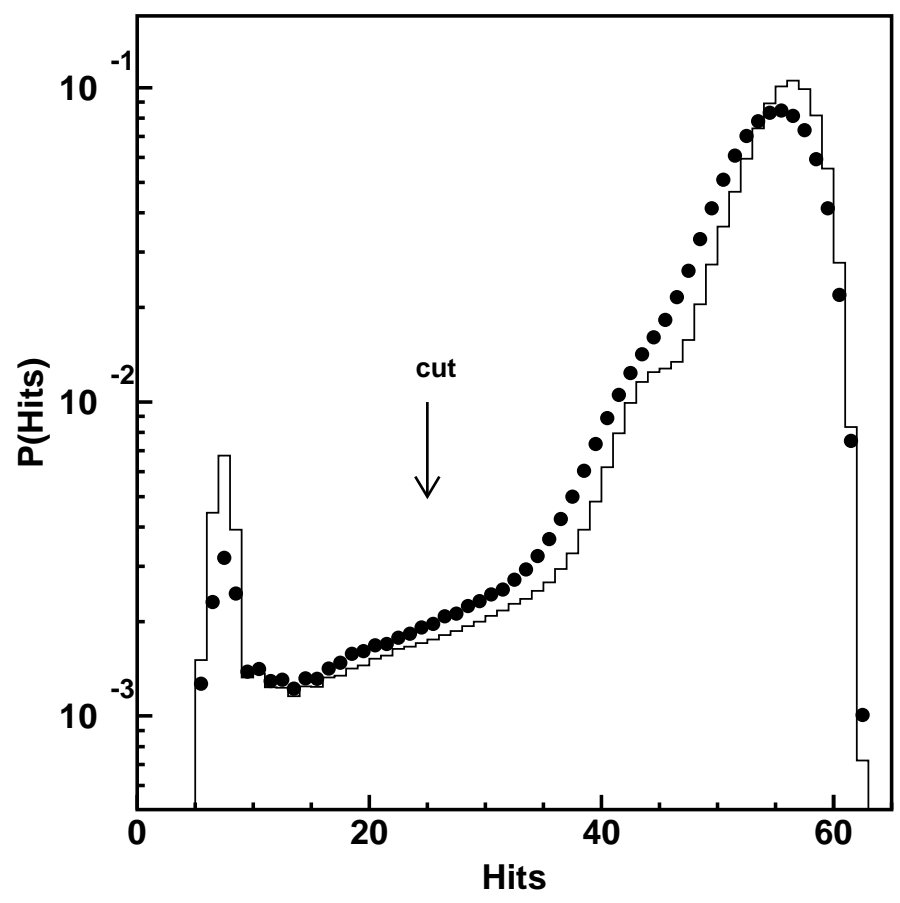

Figure 3.7: Normalized distribution of the number of TEC hits for data (dots) and JETSET (histogram). Cuts (3.6), (3.7), (3.10) and (3.11) have been applied. 


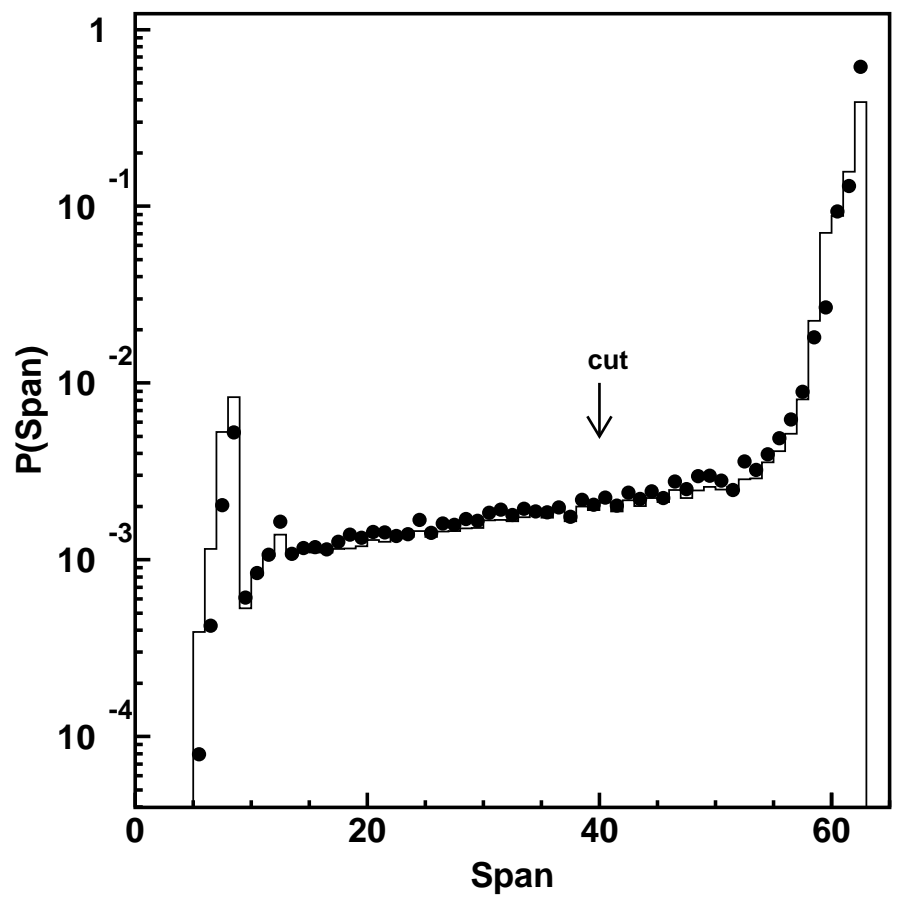

Figure 3.8: Normalized distribution of the span for data (dots) and JETSET (histogram). Cuts (3.6), (3.7), (3.10) and (3.11) have been applied.

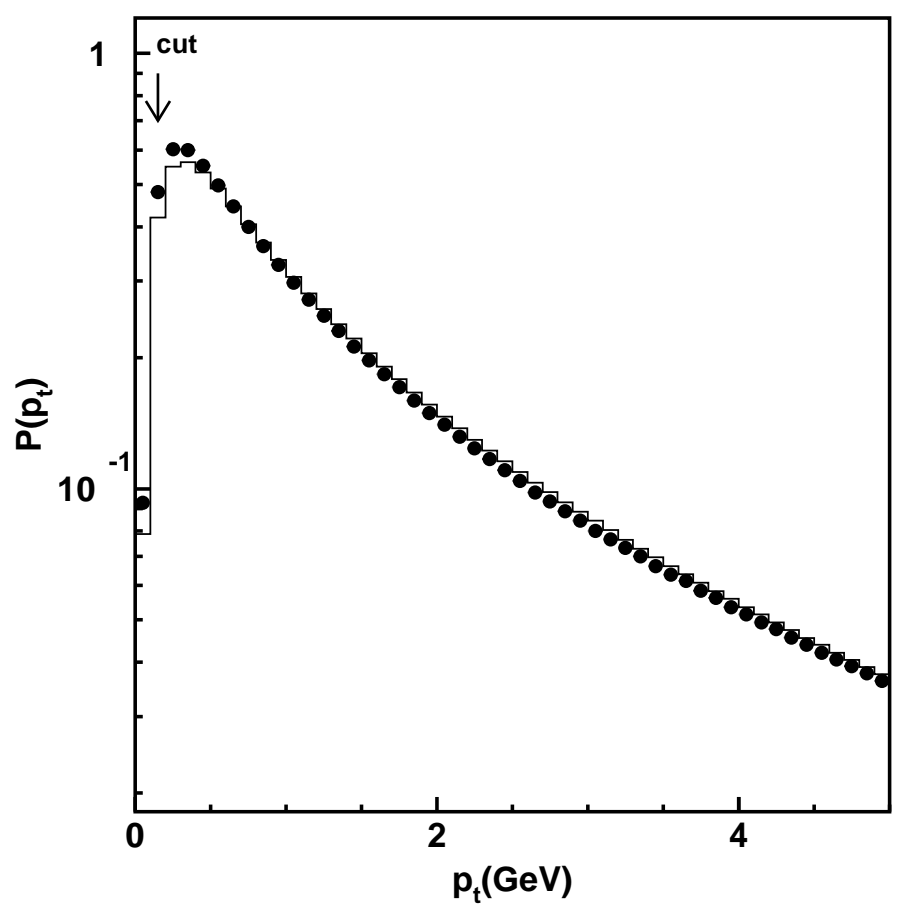

Figure 3.9: Normalized distribution of the transverse momentum of tracks for data (dots) and JETSET (histogram). Cuts (3.6), (3.7), (3.8), (3.9) and (3.11) have been applied. 


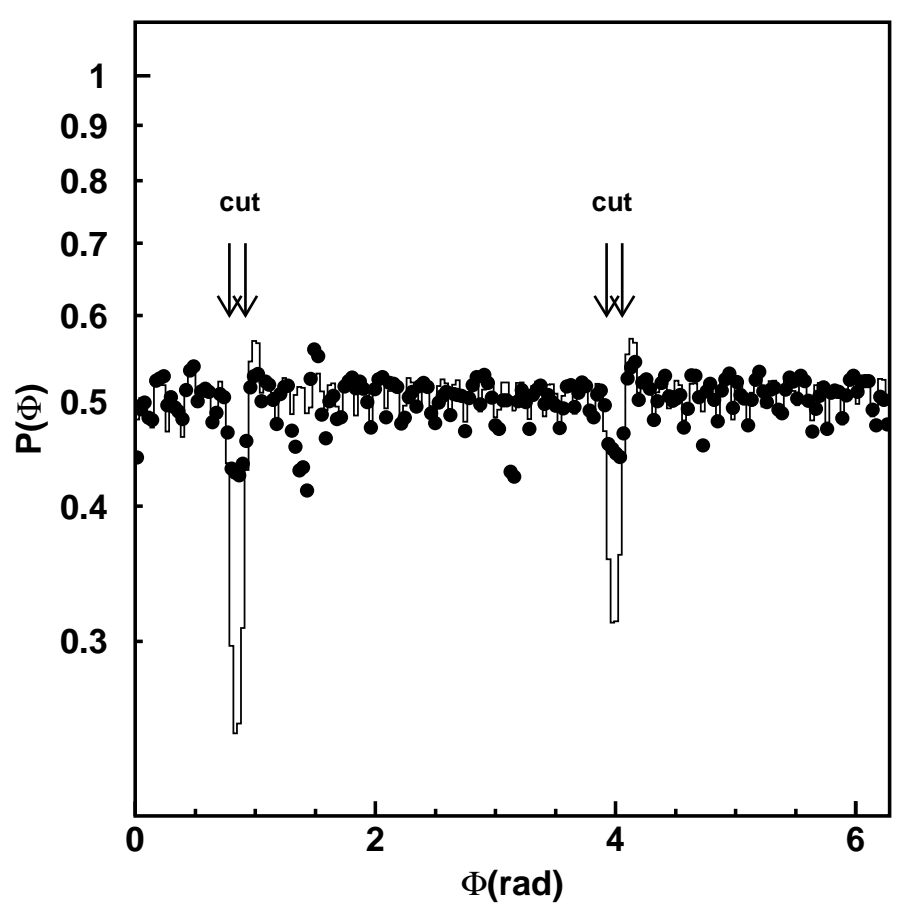

Figure 3.10: Normalized distribution of the azimuthal angle of tracks for data (dots) and JETSET (histogram). Cuts (3.6), (3.7), (3.8), (3.9) and (3.10) have been applied.

and $\mathrm{MC}$ are seen in two azimuthal angular distribution intervals: $45^{\circ}<\phi<52.5^{\circ}$ and $225^{\circ}<$ $\phi<232.5^{\circ}$ (see Fig. 3.10). Therefore, tracks located in these two regions are removed from the analysis in both data and MC samples.

\section{Two-track resolution}

Of particular importance in the study of BEC is the precision of the two-track reconstruction. For the computation of the four-momentum difference $Q$, good measurement of the opening angle between the two tracks is required, especially for low $Q$. However, when the opening angle between two like-sign tracks is less than $3^{\circ}$, the detector has great difficulty to resolve the two tracks. For this reason, an additional cut on tracks is applied to ensure good precision of the reconstruction of variables such as $Q$ and the difference in polar and azimuthal angles between two tracks $\delta \theta$ and $\delta \phi$. The additional cut requires that when no hit in the Z-chamber is found and an energy deposit in the BGO is used to recover this missing hit, the track is removed. After this additional cut, good agreement is obtained between the data and MC simulation for the difference of polar and azimuthal angle with respect to the beam direction (see Fig. 3.11), as well as for the distribution of the inclusive four-momentum difference $Q$ (see Fig. 3.12).

After all the above event and track selections, the average charged-particle multiplicity is approximately 12 out of the original 25 track candidates. 

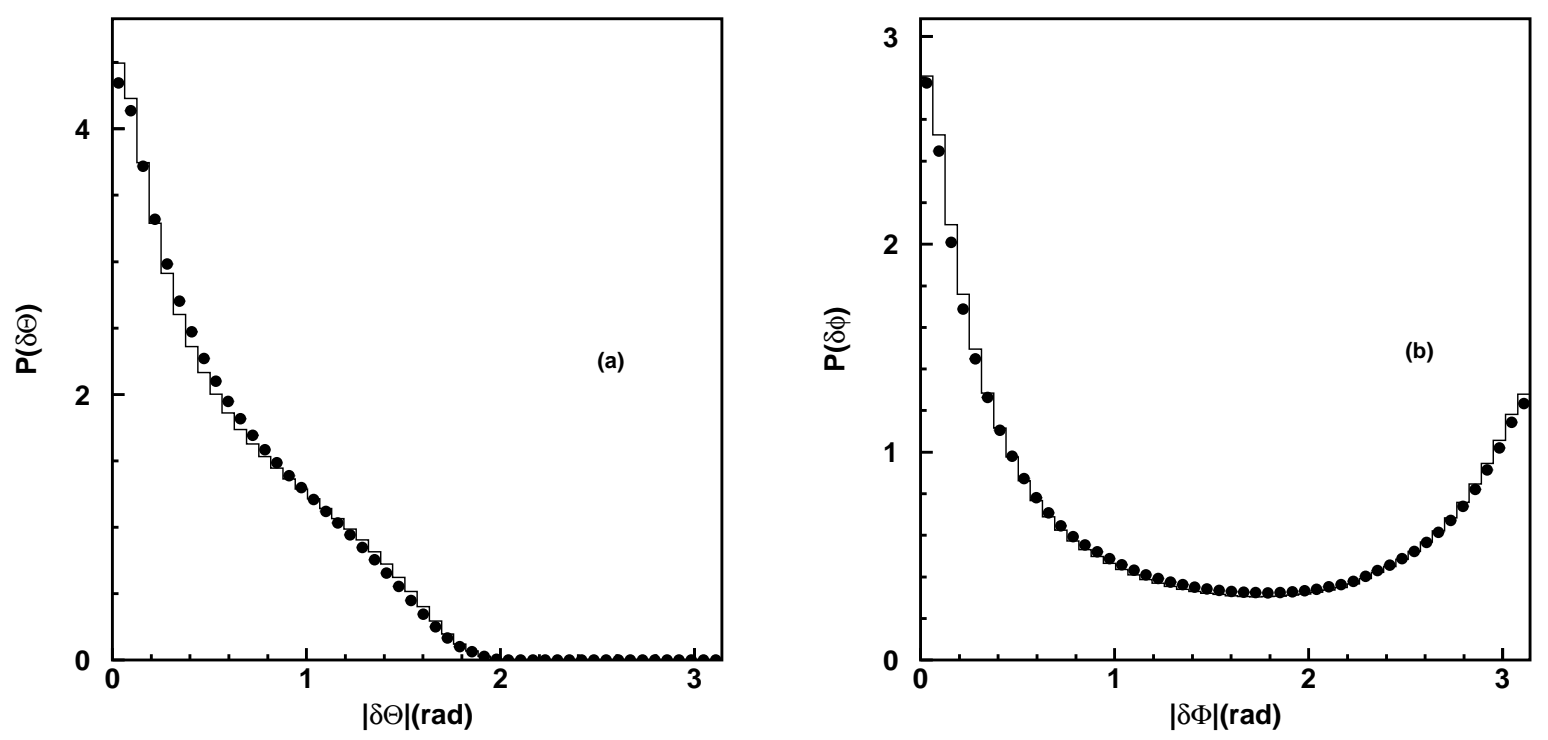

Figure 3.11: (a) The distribution of the difference between two tracks in the (a) polar (b) azimuthal angle with respect to the beam direction. Data are represented by solid dots and JETSET by the histogram. Cuts (3.6), (3.7), (3.8), (3.9), (3.10) and (3.11) have been applied. In addition, tracks with no hits in the Z-chamber are removed.

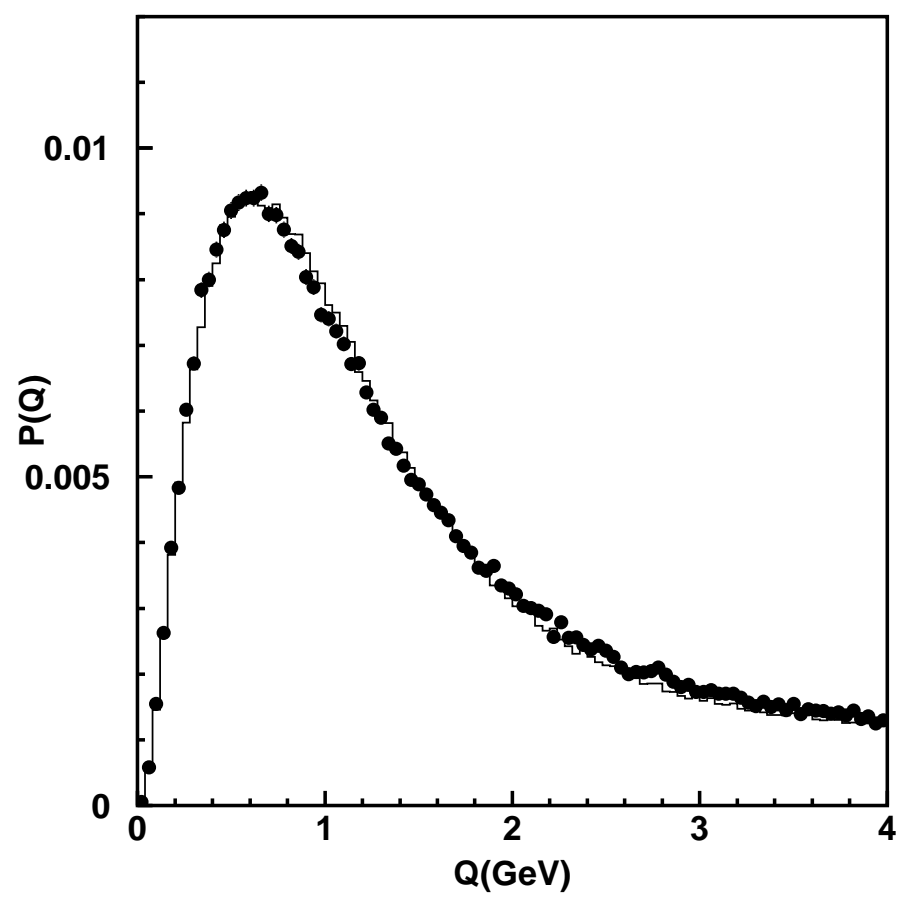

Figure 3.12: Normalized distribution of inclusive four-momentum difference $Q$. Cuts (3.6), (3.7), (3.8), (3.9), (3.10) and (3.11) have been applied. In addition, tracks with no hits in the Z-chamber are removed. 


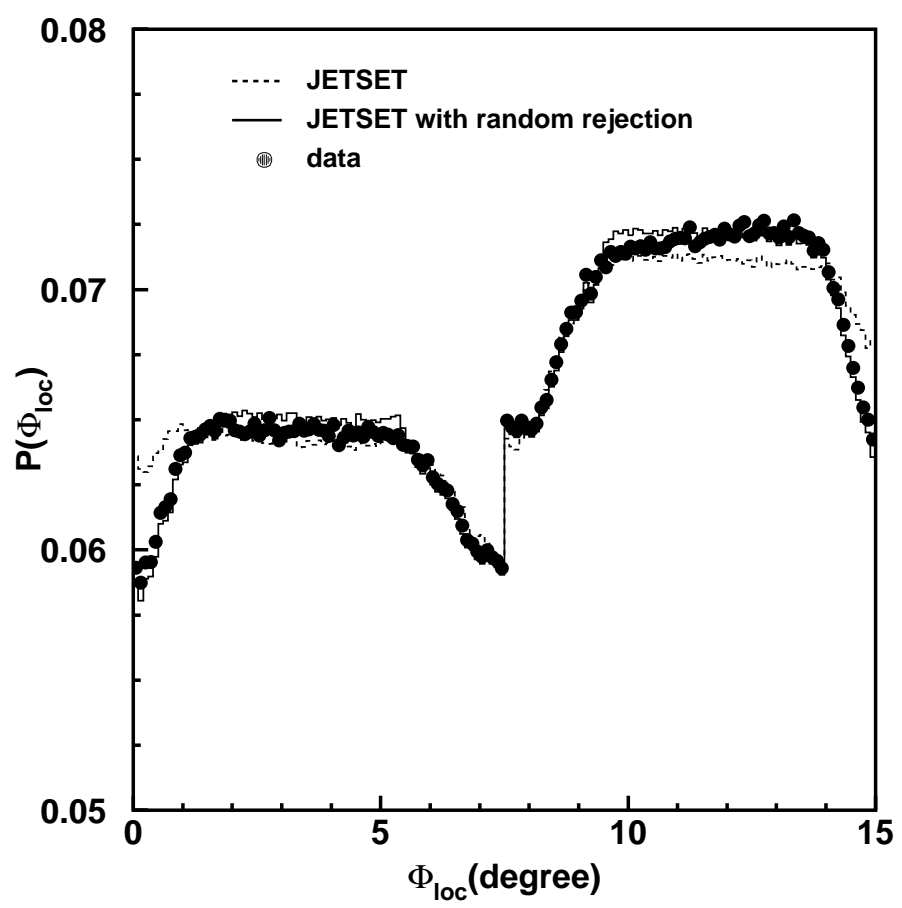

Figure 3.13: Normalized distribution of $\phi_{\text {loc }}$ for data (dots), for the JETSET at detector level (dashed line) and for JETSET at detector level with random rejection (solid line). Cuts (3.6), (3.7), (3.8), (3.9), (3.10) and (3.11) have been applied.

\subsection{TEC Inefficiencies}

During data taking, overcurrent in the anodes and cathodes occured from time to time because of high LEP background. To protect the TEC, the high voltage was then lowered or even turned off. This led to temporary loss of efficiency in some TEC sectors or the whole TEC. The Monte Carlo simulation takes the main part of this problem into account, but it turns out that the MC underestimates the inefficiency, especially the track loss close to the anodes and cathodes. This discrepancy is seen clearly from the distribution of $\phi_{\text {loc }}$, the angle between the track and the closest outer TEC anode (see Fig. 3.13).

To correct this, random rejection is applied on the MC tracks within two degrees of the anodes and cathodes. This results in a better agreement of $\phi_{\text {loc }}$ between the data and MC.

\subsection{Further Event Selection Based on Tracks}

Although event selection is based on the calorimeter clusters, additional event selection based on tracks from TEC and SMD is needed to improve the purity of hadronic events and the precision of the track measurement.

Firstly, in order to reject residual $\tau^{+} \tau^{-}$events, cuts are used on the second largest angle $\phi_{2}$ between any two neighboring tracks in the $r \phi$ plane. Selected events are required to have (see 
Fig. 3.14 (a))

$$
20^{\circ} \leq \phi_{2} \leq 170^{\circ}
$$

Secondly, in order to ensure that the tracks are in the barrel area of the TEC, the polar angle of the thrust axis $\theta_{\mathrm{thr}}^{\mathrm{TEC}}$ calculated from the tracks is required to satisfy (Fig. 3.14 (b))

$$
\left|\cos \theta_{\mathrm{thr}}^{\mathrm{TEC}}\right| \leq 0.7 .
$$

After application of the above selection criteria, 804,137 events remain with an average of about 12 tracks.
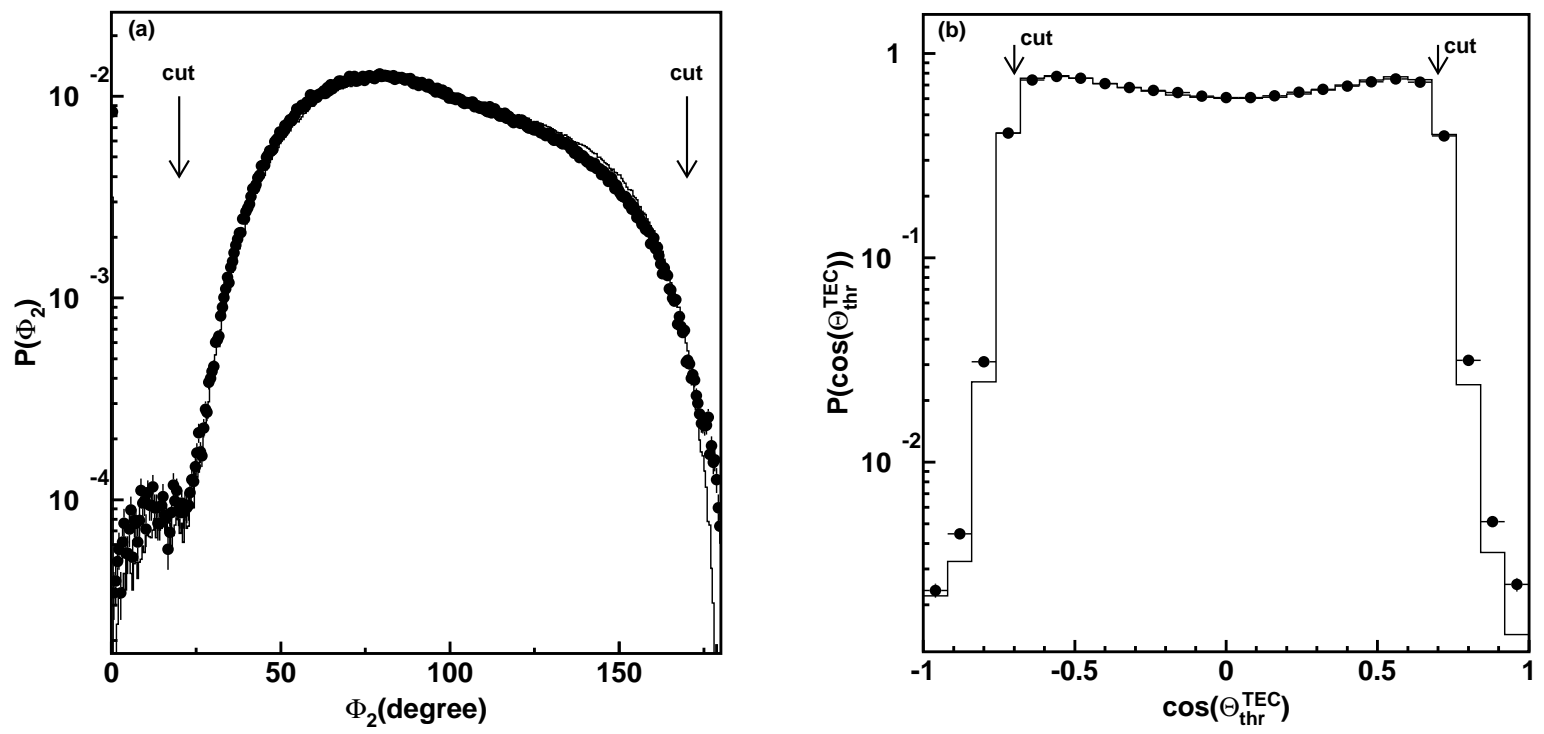

Figure 3.14: Normalized distribution of (a) $\phi_{2}$, and (b) $\cos \theta_{\mathrm{thr}}^{\mathrm{TEC}}$ for data (dots), for the JETSET (histogram). In addition to cuts (3.1), (3.2), (3.3), (3.5) and (3.4), cut (3.13) has been applied in (a) and cut (3.12) in (b).

\subsection{Light-Quark and b-Quark Event Selection}

Because of the long life-time of B mesons, particles originating from the decay of B mesons have little overlap with particles produced directly or via short-lived resonances. Consequently, BEC is greatly suppressed in b-quark events. Therefore, only light-quark (udsc) events are used in the comparison of 2-jet and 3-jet events. Anti-b-tagging is used to select light-quark events. Moreover, in order to select gluon jets with high purity, b-quark 3-jet events are chosen and b-tagging is applied to each jet separately in order to identify the gluon jet.

Anti-b-tagging and b-tagging algorithms are used separately to obtain high-purity samples of light-quark and b-quark events. The b-tagging algorithm $[35,36]$ is now briefly described.

The $\mathrm{b}$-tagging algorithm is based on the decay length and hence the lifetime of the $\mathrm{B}$ hadrons. It starts with a reconstruction of the three-dimensional primary vertex of the event based on the 
information of tracks recorded in the TEC and the SMD. To reconstruct the primary vertex, the following $\chi^{2}$ is minimized:

$$
\chi_{N_{\text {trk }}}^{2}=\sum_{i=1}^{N_{\text {trk }}}\left(\vec{t}_{i}-\vec{f}\left(\vec{v}, \vec{q}_{i}\right)\right)^{T} G_{i}^{-1}\left(\vec{t}_{i}-\vec{f}\left(\vec{v}, \vec{q}_{i}\right)\right)+\left(\vec{v}-\vec{v}_{\text {fill }}\right)^{T} V_{\text {fill }}^{-1}\left(\vec{v}-\vec{v}_{\text {fill }}\right)
$$

where $N_{\text {trk }}$ is the number of tracks, $\vec{t}_{i}$ is the vector of the set of measured parameters for the $i^{\text {th }}$ track, $G_{i}$ is the corresponding covariance matrix, $\vec{f}\left(\vec{v}, \vec{q}_{i}\right)$ is the predicted measurement assuming the track has originated from the vertex $\vec{v}$ with momentum $\vec{q}_{i}, \vec{v}_{\text {fill }}$ is the so-called fill or $N$-event vertex, which is the mean event vertex in $N$ events obtained by minimizing $\chi^{2}=\frac{d_{r \phi}^{2}}{\sigma_{r \phi}^{2}}$, where $d_{r \phi}$ is the DCA in the $x y$ plane and $\sigma_{r \phi}$ the error on the DCA, and $V_{\text {fill }}$ is a diagonal matrix describing the size of the beam spot. The $N_{\text {trk }}$ tracks used in the calculation of $\chi_{N_{\text {trk }}}^{2}$ is required to satisfy the following criteria:

- fitted with a Kalman filter [37], which updates the track trajectory at each measurement plane of SMD using the error propagation package GEANE [38], which is based on the detector simulation program GEANT;

- $\left|d_{r \phi}\right|<\min \left(10 \mathrm{~mm}, 5 \sigma_{r \phi}\right)$;

- $\left|d_{s z}\right|<100 \mathrm{~mm}$, where $d_{s z}$ is the DCA in the $s z$ plane (see Fig. 3.15).

If the confidence level $P\left(\chi_{N_{\text {trk }}}^{2}\right)$ is below $4 \%$ for an event, tracks are removed one by one and $\chi_{N_{\text {trk }}-1, i}^{2}$ is calculated with track $i$ is removed. This results in the confidence level $P_{i}=P\left(\chi_{N_{\text {trk }}}^{2}-\right.$ $\left.\chi_{N_{\text {trk }}-1, i}^{2}\right)$. If either $P\left(\chi_{N_{\text {trk }}}^{2}\right)$ is below $4 \%$ or any $P_{i}$ is below $2 \%$, the track with lowest $P_{i}$ is removed. The iterative procedure continues until no more tracks need to be removed. Only primary vertices with at least 3 tracks are kept.

Once the primary vertex is reconstructed, it can be used to determine the track's DCA in the $r \phi$ plane, $d_{r \phi}$, and $s z$ plane, $d_{s z}$ (see Fig. 3.15). These distances are signed positive if the track crosses the jet axis in the direction of the jet, negative if the backwards extrapolation of the track crosses the jet axis. The decay length of the track, which is the distance between the primary vertex and the crossing point of the track with the jet axis, is determined in both $r \phi\left(L_{r \phi}\right)$ and $s z\left(L_{s z}\right)$ planes and given a sign according to the same criteria as for DCA. These projected decay lengths represent independent measurements of the true decay length of the B hadron and their weighted average can be taken to form the decay length variable $L$. Tracks from the decay of long lifetime particles such as the B hadron usually have a large and positive-signed decay length.

Although the two projections $L_{r \phi}$ and $L_{s z}$ both have good potential to measure the decay length, the $r \phi$ measurements usually dominate because the SMD and the TEC have better resolution in that direction. In the case when the track is at a small angle to the jet direction in the $r \phi$ projection, the $r \phi$ measurement is not useful and the $s z$ decay length measurement becomes important. If the confidence level that both $r \phi$ and $s z$ measurements are compatible with each other is larger than $5 \%$, the two measurements are averaged. Otherwise, only the $r \phi$ projection is used to reduce the effect from the errors in the $s z$ pattern recognition.

For tracks which are assumed to have originated from the decay of particles with no discernible lifetime, the probability that a given track originates from the primary vertex is estimated from the distribution of decay length significances, $S=L / \sigma_{L}$, where $\sigma_{L}$ is the error on decay length 


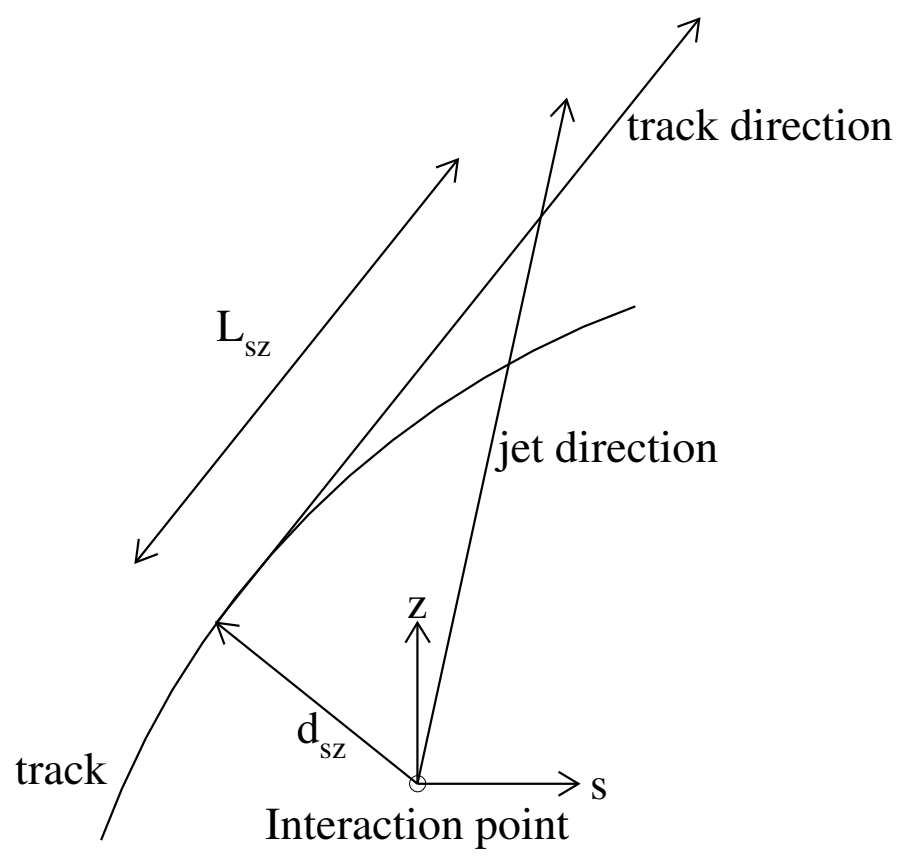

Figure 3.15: DCA of a track, $d_{s z}$, and its decay length, $L_{s z}$, in the $s z$ plane.

$L$. The combination of decay length information from more than one track in the event can be facilitated with the probability. The assumptions are made that the tracks with negative $S$ originate from the primary vertex and that the probability density $f(S)$ is symmetric around zero. Thus the negative side of the significance distribution can be used to determine the probability density function $f(S)$. The probability that a track with significance $S$ and positive decay length originates from the primary vertex is

$$
P(S)=\frac{\int_{S}^{\infty} f\left(S^{\prime}\right) \mathrm{d} S^{\prime}}{\int_{0}^{\infty} f\left(S^{\prime}\right) \mathrm{d} S^{\prime}}
$$

A track originating from a b-quark will have a large significance $S$ and thus a small $P(S)$.

By defining the probability for any negative decay length track to be equal to a track with zero decay length, i.e., $P(S<0) \equiv 1$, the track probabilities can be combined to form the probability of the event, $P_{\text {event }}$, which contains the information on whether an event is a b-quark event,

$$
P_{\text {event }}=\frac{\prod_{j=1}^{N_{+}} P\left(S_{j}\right)}{2^{N}} \sum_{i=0}^{N-1} \sum_{j=i+1}^{N} C_{j}^{N} \frac{\left(-\ln \prod_{j=1}^{N_{+}} P\left(S_{j}\right)\right)^{i}}{i !}
$$

where $N$ is the total number of tracks in the event and $N_{+}$is the number of tracks with positive decay length. $P_{\text {event }}$ is close to 0 for b-quark events, while $P_{\text {event }}$ is larger for other types of events. To emphasize the charateristic of b-quark events, the discriminant $\delta_{\text {event }}$ is defined as

$$
\delta_{\text {event }}=-\log _{10} P_{\text {event }} .
$$




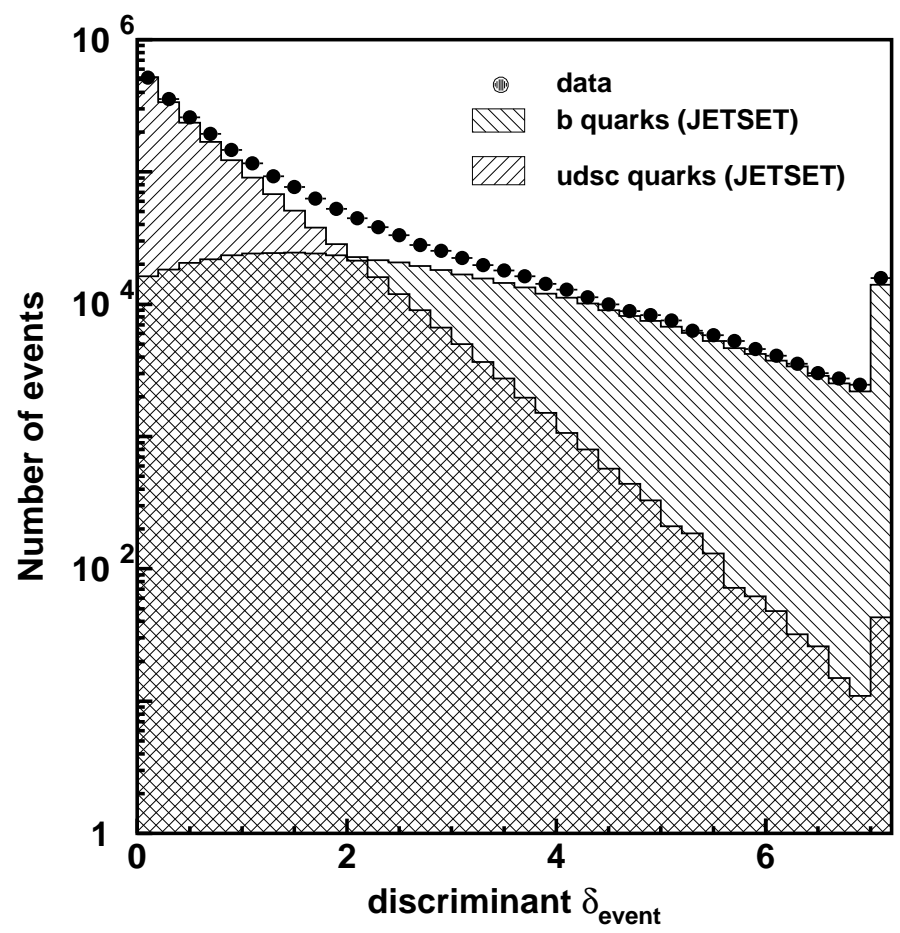

Figure 3.16: Event discriminant distribution for the 1994 data, compared to the discriminant distribution of the Monte Carlo for b-quark events and light-quark events.

The event distribution of this discriminant is shown in Fig. 3.16 for the 1994 data and for JETSET. The discriminant of the data is for all events and that of JETSET is for light-quark and b-quark events, separately. We see that for JETSET b-quark events dominate the large- $\delta_{\text {event }}$ region whereas light-quark events dominate the small- $\delta_{\text {event }}$ region. This gives us confidence in the use of this discriminant to select light-quark and b-quark events.

Events are classified as light-quark events if $\delta_{\text {event }}<\delta_{\text {event }}^{c}$ and as b-quark events if $\delta_{\text {event }}>$ $\delta_{\text {event }}^{\mathrm{c}}$. Fig. 3.17 shows the purity and efficiency distributions as a function of $\delta_{\text {event }}^{\text {c }}$ for JETSET of light-quark events and b-quark events in the light-quark samples (Fig. 3.17(a)) and in the b-quark samples (Fig. 3.17(b)).

We need light-quark and b-quark samples with high purity. Light-quark events are selected with a discriminant value $\delta_{\text {event }}^{\mathrm{c}}=1.3$. For this cut, the purity and efficiency of the light-quark sample are $92.9 \%$ and $84.6 \%$, respectively. The b-quark events are selected with $\delta_{\text {event }}^{\text {c }}=2.5$, resulting in a purity and efficiency of $84.3 \%$ and $51.1 \%$, respectively. Here the purity and efficiency are defined as

$$
\begin{gathered}
\text { purity }=\frac{\text { number of real light-quark (or b-quark) events after } b \text { tag }}{\text { number of light-quark (or b-quark) events selected after b tag }} \\
\text { efficiency }=\frac{\text { number of real light-quark (or b-quark) events after b tag }}{\text { number of real light-quark (or b-quark) events }} .
\end{gathered}
$$



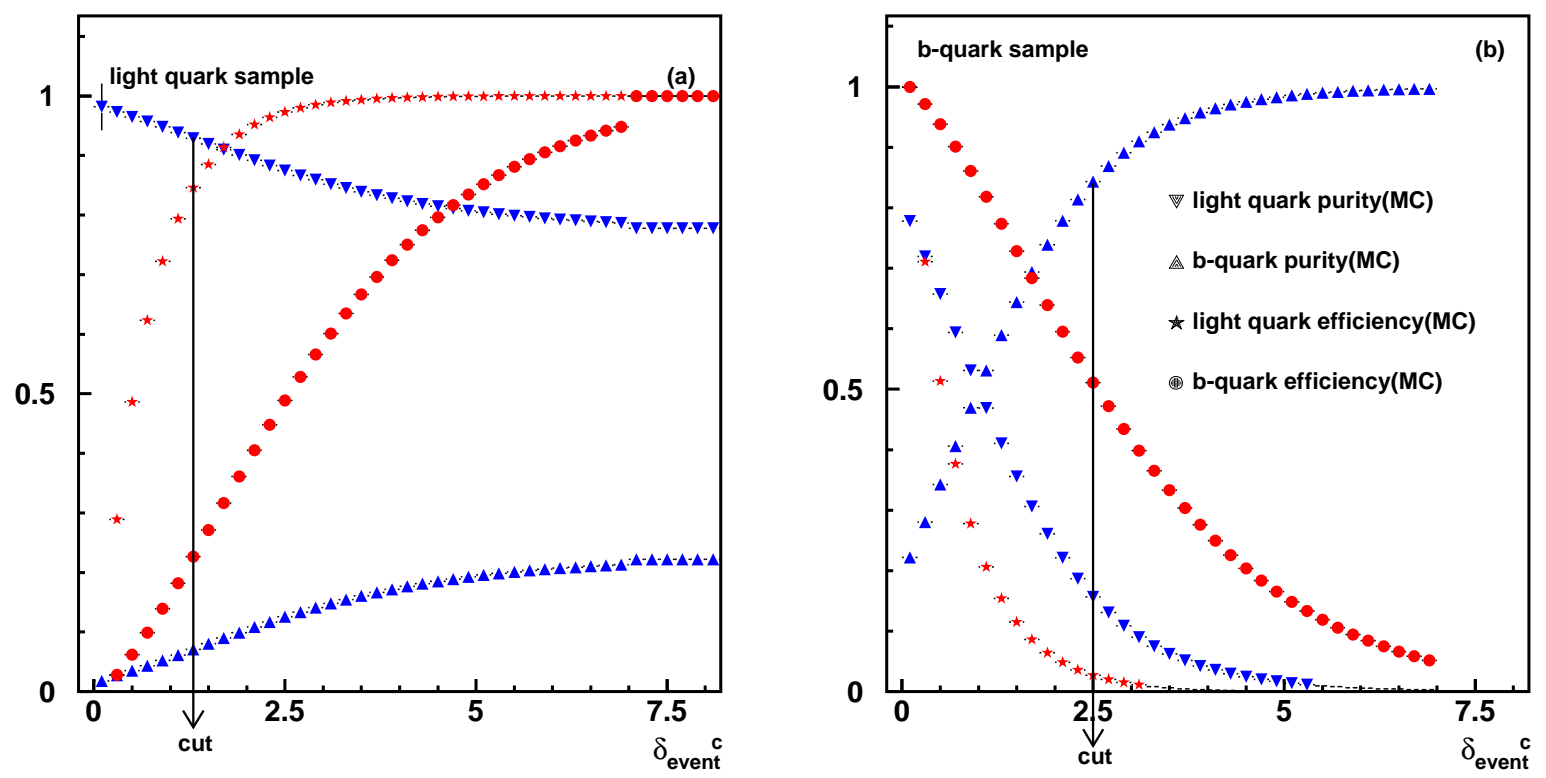

Figure 3.17: The purity and efficiency as a function of $\delta_{\text {event }}^{\mathrm{c}}$ for JETSET of light-quark events and b-quark events in the light-quark sample (a) and in the b-quark sample (b).

\subsection{Jet Clustering Algorithm}

There are several jet clustering algorithms, e.g., the JADE [39, 40] and Durham [41, 42] algorithms, which are widely used in high energy physics studies. The Durham algorithm is used in this thesis. It has the advantage of a better treatment of low energy particles, which are particularly important for BEC.

The Durham algorithm is iterative, beginning with a list of jets that are just the observed particles themselves. At each stage of iteration, the following expression is evaluated for each pair of jets $i$ and $j$ :

$$
y_{i j}=\frac{2 \min \left(E_{i}^{2}, E_{j}^{2}\right)}{\left(\sum_{k} E_{k}\right)^{2}}\left(1-\cos \theta_{i j}\right),
$$

where $E_{i}$ and $E_{j}$ are their energies and $\theta_{i j}$ is the angle between them. The pair $i, j$ which has the smallest value of $y_{i j}$ and has $y_{i j}$ below the jet resolution parameter $y_{\text {cut }}$ is combined into a new jet $l$ with four-momentum

$$
p_{l}^{\mu}=p_{i}^{\mu}+p_{j}^{\mu}
$$

This procedure is repeated until all the $y_{i j}$, calculated using the remaining jets and the combined new jets, exceed the jet resolution parameter $y_{\text {cut }}$. In this way, each event is classified as containing two, three, four, ... jets, where the number of jets depends on the value of $y_{\text {cut }}$.

The jet algorithm is applied using calorimeter clusters, thus including neutral as well as charged particles. The charged particles are then assigned to the nearest (in angle) jet.

In order to see whether BEC in light-quark 2- and 3-jet events are $y_{\text {cut }}$ dependent, $y_{\text {cut }}$ is chosen 


\begin{tabular}{|ccccc|}
\hline$y_{\text {cut }}$ & 2-jet fraction & 3-jet fraction & 4-jet fraction & 5-jet fraction \\
\hline 0.005 & $52 \%$ & $36 \%$ & $10 \%$ & $1.5 \%$ \\
0.006 & $56 \%$ & $34 \%$ & $8 \%$ & $1 \%$ \\
0.01 & $65 \%$ & $29 \%$ & $5 \%$ & $0.3 \%$ \\
0.02 & $76 \%$ & $22 \%$ & $2 \%$ & \\
1 & $100 \%$ & & & \\
\hline
\end{tabular}

Table 3.1: The fraction of light-quark data events classified as 2-jet, 3-jet, 4-jet, 5-jet events as a function of the jet resolution parameter $y_{\text {cut }}$, using the Durham algorithm.

to be $0.005,0.006,0.01,0.02$ and 1 in the following analysis. The fractions of light-quark 2-jet and 3 -jet events (using experimental data) as a function of the jet resolution parameter $y_{\text {cut }}$ are shown in Table 3.1.

\subsection{Selection of Gluon Jets and Quark Jets}

First, events are separated into 2-jet and 3-jet samples. Various values of $y_{\text {cut }}$ are used in this thesis. Here the selection of quark and gluon jets is illustrated using $y_{\text {cut }}=0.02$, which is the value used most often in this thesis.

Since BEC are suppressed in b-quark jets, we use quark jets from light-quark 2-jet and 3-jet events, in the latter case assuming the least energetic jet to be the gluon.

Gluon jets are taken from light-quark or b-quark 3-jet events. Two methods are used to select the gluon jet:

1. Energy rank: the least energetic jet in the light-quark 3-jet event is chosen as the gluon jet. The purity of this gluon jet sample is $68 \%$. The purity decreases with increasing gluon jet energy. The gluon purity is defined here as

$$
\text { purity }=\frac{\text { the number of real gluon jets in the light-quark gluon jet sample }}{\text { the number of light-quark } 3 \text {-jet events }} .
$$

2. Double b-tagging: Firstly, event b-tagging is used to select b-quark events, which results in a purity and efficiency of b-quark events of $84 \%$ and $51 \%$ (see Fig. 3.18(a)).

Secondly, jet b-tagging is used in the 3 -jet b-quark events to identify the gluon jet. A discriminant, $\delta_{\text {jet }}$, is calculated for each jet separately, using the same algorithm as for b-event tagging except that only particles belonging to the jet are used. We have tried two ways for the jet b-tagging:

(a) Jet b-tagging on 3 jets: Two jets are required to have $\delta_{\text {jet }}>\delta_{\text {jet }}^{\text {c }}$ and one jet to have $\delta_{\text {jet }}<\delta_{\text {jet }}^{\mathrm{c}}$. The jet with $\delta_{\text {jet }}<\delta_{\text {jet }}^{\mathrm{c}}$ is selected as the gluon jet.

(b) Jet b-tagging on 2 jets: Jet 1, the most energetic jet, is assumed to be a quark. Of the two least energetic jets one is required to have $\delta_{\text {jet }}>\delta_{\text {jet }}^{\mathrm{c}}$ and one to have $\delta_{\text {jet }}<\delta_{\text {jet }}^{\mathrm{c}}$. The latter is selected as the gluon jet. 

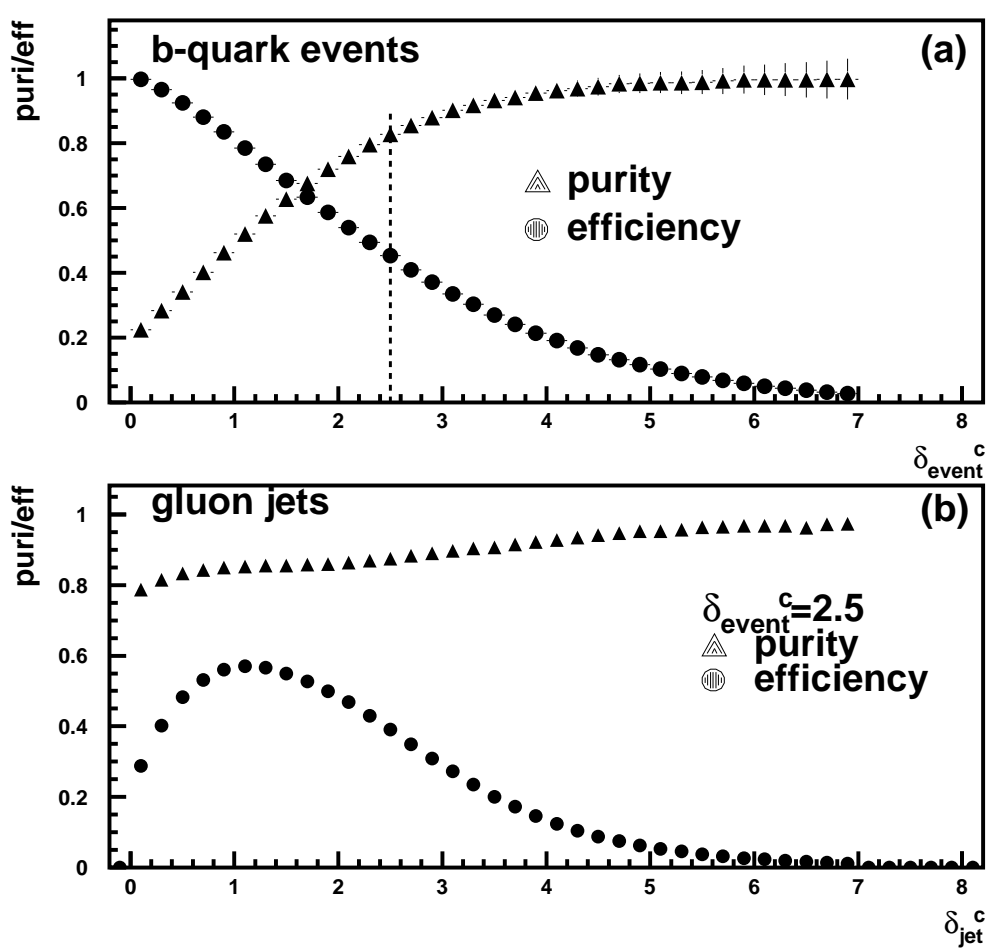

Figure 3.18: (a) Purity and efficiency of b-quark events vs. $\delta_{\text {event }}^{\text {c }}$ (b) Purity and efficiency of gluon jets vs. $\delta_{\text {jet }}^{\mathrm{c}}$ for $\delta_{\text {event }}^{\mathrm{c}}=2.5$. JETSET is used to estimate the purity and efficiency.

Since method (2a) results in slightly higher purity and efficiency, we choose to use that method in almost all b-quark 3-jet samples. However, for Mercedes (Sect. 3.8.1) and Yg (Sect. 3.8.3) events we use method (2b).

With $\delta_{\text {jet }}^{\text {c }}=0.8$, we obtain 10,711 gluon jets with $84 \%$ purity and an efficiency of $53 \%$ (see Fig. 3.18(b)), where the purity and efficiency are defined as

$$
\begin{gathered}
\text { purity }=\frac{\text { the number of real gluon jets in the gluon jet sample }}{\text { the number of jets in the gluon jet sample }} \\
\text { efficiency }=\frac{\text { the number of real gluon jets in the gluon jet sample }}{\text { the number of b-quark events }} .
\end{gathered}
$$

In the light-quark events, the purity and efficiency of gluon jets are estimated using JETSET $\mathrm{ME}^{1}$ at generator level. The real gluon jets are judged using the following method: There are 3 partons and 3 jets (using the Durham algorithm). There are 6 combinations in matching the 3 partons and 3 jets. The sum of the angles between the partons and jets is calculated for each of the 6 combinations. The combination with the smallest sum is that in which the directions of the three jets best match those of the three partons. If the gluon jet (least energetic jet) is along the gluon parton direction in this combination, then this jet corresponds to a real gluon.

\footnotetext{
${ }^{1}$ For a description of Monte Carlo event generators see Chapter 4.
} 
In the heavy-quark events, the purity and efficiency of gluon jets are estimated using JETSET PS on the detector level. Jets are reconstructed using the Durham algorithm and the gluon jet chosen using b-tagging. Whether this jet is really a gluon jet is judged in this way: The information on whether a produced charged particle is the decay product of a $b$ quark can be traced back in the generator. For each jet, the sum of the energies of the charged particles originating from the $b$ quark is calculated. The real gluon jet is taken as the one with the smallest sum of energies.

Since purity and efficiency are only used to help to choose the cuts, it is not unreasonable to do it on the generator level. The main reason the generator level is used to estimate the purity and efficiency of the gluon jet in light-quark events is that the gluon parton directions are not available at detector level.

\subsection{Special 3-jet Topologies: Mercedes, Yq, and Yg Events}

To obtain samples of quark and gluon jets having similar energies, some special 3-jet configurations are used.

Since the three jets of the 3-jet event are confined to a plane by energy-momentum conservation, their configuration can be fully specified by the three inter-jet angles. Events where all three angles are equal are referred to as Mercedes events; those where two of the angles are equal as $\mathrm{Y}$ events.

Heavy-quark data and MC detector level events are first forced to be co-planar by subtracting $1 / 3$ of the transverse momentum imbalance from each of the three jets.

\subsubsection{Mercedes events}

So-called Mercedes events are required to have the angles between each pair of jets equal to $120^{\circ}$ within a certain angular tolerance $\theta$. The gluon jet in Mercedes events is selected using jet btagging on all 3 jets. The purity and efficiency distributions of gluon jets in the Mercedes events as a function of $\theta$ is shown in Fig. 3.19.

In the analysis, Mercedes events are selected as 3-jet events with the angles between each pair of jets being $120^{\circ}$ within $15^{\circ}$, which provides reasonable purity and efficiency while still keeping the energies of the three jets approximately equal.

\subsubsection{Yq events}

So-called $Y$ events are selected by requiring both $\theta_{2}$ and $\theta_{3}$ (Fig. 3.20) to be $150^{\circ}$ within an angular tolerance $\theta$. Yq events are defined as Y events where the most energetic jet (jet 1) is a quark jet. The purity and efficiency distributions of gluon jets in the Yq events as a function of angular tolerance $\theta$ is shown in Fig. 3.21.

In this analysis the tolerance $\theta$ is chosen to be $15^{\circ}$, which provides a sample with reasonable purity and efficiency. 

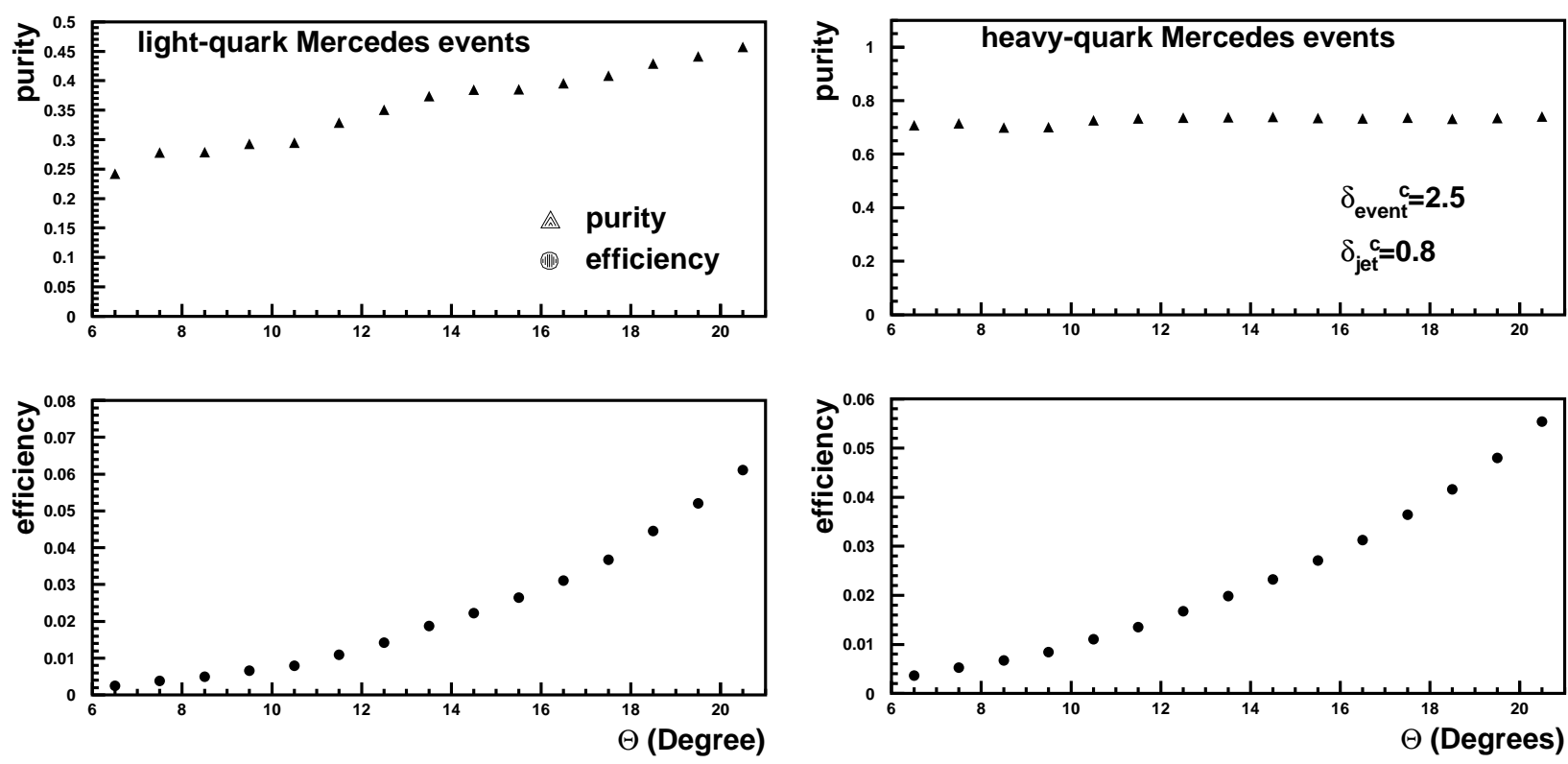

Figure 3.19: The purity (above) and efficiency (below) of gluon jets in the light-quark (left) and heavy-quark (right) Mercedes events as a function of the angular tolerance between jets. The purity and efficiency of the gluon in the light-quark Mercedes events are estimated using JETSET at generator level, and in the heavy-quark Mercedes events using JETSET at detector level.

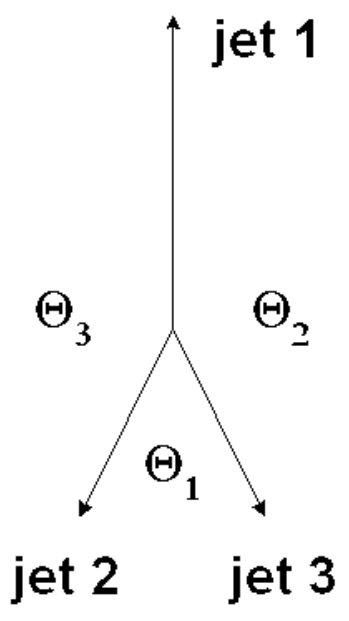

Figure 3.20: Illustration of the angles between the jets in Yq and Yg events. For Yq events jet 1 is a quark and for Yg events jet 1 is a gluon. 

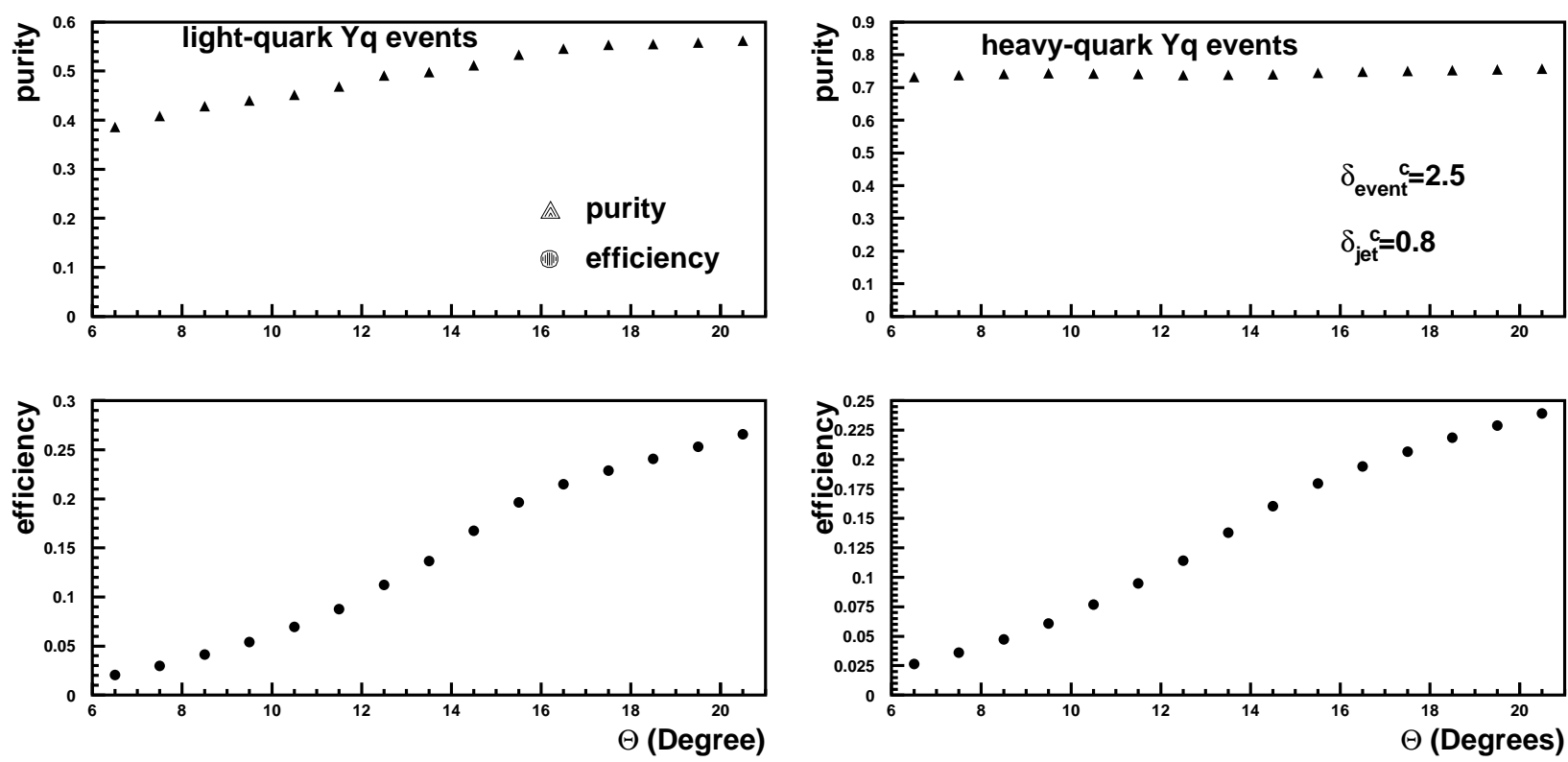

Figure 3.21: The purity (above) and efficiency (below) of gluon jets in the light-quark (left) and heavy-quark (right) Yq events as a function of the angular tolerance between jets. The purity and efficiency of gluon in the light-quark Yq events are estimated using JETSET at generator level, and those in the heavy-quark Yq events are estimated using JETSET at detector level.

\subsubsection{Yg Events}

So-called Yg events are required to have the angles between the gluon jet and the two quark jets $\left(\theta_{2}\right.$ and $\theta_{3}$ in Fig. 3.20) both larger than an angle $\Theta$ [43]. Further, the gluon jet is selected using jet b-tagging on all three jets and its energy is required to be larger than that of the other two jets.

The purity and efficiency of the selected gluon jets and given in Fig. 3.22 as a function of the angle $\Theta$ varying from $90^{\circ}$ to $160^{\circ}$. On the basis of these distributions, $\Theta=100^{\circ}$ is chosen, thus obtaining a reasonable purity and efficiency for the selected gluon jets. 

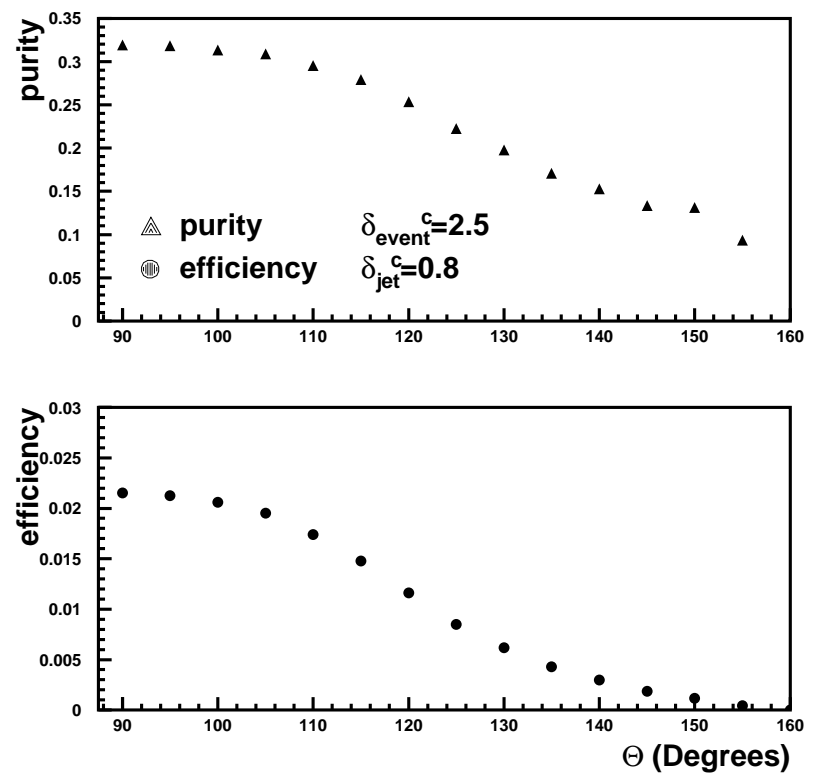

Figure 3.22: The purity and efficiency of gluon jets in Yg events as a function of $\Theta$. 



\section{Chapter 4}

\section{Monte Carlo Generators}

Monte Carlo generators are essential for at several stages of the analysis. Firstly, the correlation function $R_{2}$ is measured using Monte Carlo events without BEC as a reference sample, or using mixed events (see Sect. 5.2). In the latter case, the mixing procedure removes more correlations than just BEC, which is corrected by a factor determined using Monte Carlo without BEC. Secondly, corrections to $R_{2}$ for limited detector resolution, acceptance, efficiency and particle identification are determined using Monte Carlo events passed through the L3 detector simulation (see Sect. 2.5). Thirdly, purity and efficiency of light-quark and heavy-quark events are estimated by comparing Monte Carlo samples at the generator level and at the simulated detector level. Fourthly, the purity of the gluon jet sample is estimated on the Monte Carlo generator level.

In this chapter, the four phases of the hadron production process in $\mathrm{e}^{+} \mathrm{e}^{-}$collisions is briefly described in Sect. 4.1. The implementation of the hadronic process in four Monte Carlo generators is treated in Sect. 4.2. Finally, alternative ways of implementing BEC in Monte Carlo generators are discussed in Sect. 4.3.

\subsection{Hadron Production in $\mathrm{e}^{+} \mathrm{e}^{-}$Collisions}

Electron-positron scattering is one of the basic tools to study the QCD properties of multiparticle processes at high energies. At the center-of-mass energy of $\sqrt{\mathrm{s}} \approx 91.2 \mathrm{GeV}$, the multiparticle production process $\mathrm{e}^{+} \mathrm{e}^{-} \rightarrow \mathrm{q} \overline{\mathrm{q}} \rightarrow$ hadrons is dominated by $s$-channel $\mathrm{Z}$ exchange. Monte Carlo programs simulate the hadronic process by factorizing it into four different phases:

1. The $\mathrm{e}^{+} \mathrm{e}^{-}$pair annihilates into a virtual $\gamma^{*} / \mathrm{Z}$ and this decays into the $\mathrm{q} \overline{\mathrm{q}}$ pair according to electroweak theory.

2. The primary q $\bar{q}$ pair produced in phase 1 may radiate gluons, which in turn may split into $q \bar{q}$ pairs or radiate additional gluons. This phase can be described by perturbative QCD using two alternative approaches.

One approach is the matrix element (ME) method. Here, Feynman diagrams are calculated exactly, order by order, in the strong coupling constant $\alpha_{\mathrm{s}}$. However, the calculation becomes 
increasingly difficult for higher orders. Consequently, the matrix element method is only available for a maximum of four partons in the final state.

The other approach is called the parton shower (PS) method. It is based on an approximation of the full matrix element expression. Each parton produced in the initial hard process splits into two partons according to three possible branchings, $\mathrm{q} \rightarrow \mathrm{qg}, \mathrm{g} \rightarrow \mathrm{gg}$ and $\mathrm{g} \rightarrow \mathrm{q} \overline{\mathrm{q}}$. Also the newly produced partons split. This results in a multi-jet event with no explicit upper limit on the number of partons involved. This method is based on the framework of the Modified Leading Logarithmic Approximation (MLLA) [44, 45]. In the Leading Logarithmic Approximation (LLA) [13,46-49] only the leading logarithmic terms in the perturbative expansion are kept. In the MLLA some aspects of the Next-to-Leading Logarithmic Approximation (NLLA) [50-52] are also included. The Monte Carlo programs also impose energymomentum conservation at each splitting, a feature which goes beyond these approximation schemes.

3. Hadronization of quarks and gluons. The colored partons produced in phase 2 combine to form hadrons. This transition must take place since QCD leads to color confinement and no free parton can be observed. Because perturbative QCD calculations are not valid at low energy scales, the hadronization of partons can not be described by perturbative QCD. So this phase is normally explained by phenomenological models. The separation between the perturbative and hadronization phases is generally characterised by an energy scale of 1-2 GeV. There are three main types of phenomenological model used today: independent fragmentation[53], string fragmentation[54, 55] and cluster fragmentation[56].

The independent fragmentation model assumes that partons fragment independently. In this scheme, high momentum quarks evolve separately, splitting into colorless particles and other quarks. This model is designed to reproduce the limited transverse momenta and has the great advantage of simplicity. However, it fails to describe certain experimental data[57, 58] and is not used in this thesis.

The string model is derived from the QCD inspired idea that a color flux tube (string) is stretched between quark-antiquark pairs, with a gluon corresponding to a kink in the string, as described in Sect. 1.2.

In the cluster model, gluons from the perturbative phase are first split into quark-antiquark pairs. The quarks and antiquarks are then locally grouped into colorless clusters which, depending on their mass, decay into lower mass clusters or directly into hadrons.

4. Decay of unstable particles. The unstable hadrons produced in phase 3 decay according to the experimentally known branching ratios.

These different perturbative QCD approaches and fragmentation models are incorporated into different Monte Carlo programs. Here, I briefly describe the four Monte Carlo programs used in the analysis: JETSET 7.4[59], PYTHIA 6[60], HERWIG 5.9[61], HERWIG 6[62], and ARIADNE 4.06[63]. 


\subsection{Four Monte Carlo Generators}

JETSET 7.4 contains two options for the simulation of a hard process. One is the parton shower (PS) approach, the other the matrix element (ME) approach.

The JETSET 7.4 PS Monte Carlo program and its successor PYTHIA simulate $\mathrm{e}^{+} \mathrm{e}^{-}$annihilation into $q \bar{q}$ pairs and the subsequent quark and gluon branchings using a parton shower based on the MLLA framework. Initial-state radiation (ISR) is included in JETSET using the lowest order calculation. In PYTHIA an 'initial-state shower' is used to simulate ISR. These two programs contain both string and independent fragmentation options. We use string fragmentation with the Peterson fragmentation function [64] for heavy quarks (c,b) and the Lund symmetric function [65] for light quarks (u,d,s).

In JETSET 7.4 ME, the partons are generated according to exact second-order matrix elements, which provide up to four partons. A suitable renormalization scale is chosen according to the "optimal perturbative theory" [66], in order to partially compensate the higher-order terms missing in the second-order calculation. The fragmentation process is the same as in JETSET PS.

The HERWIG Monte Carlo program, like JETSET, uses an MLLA approach to simulate a parton shower. However the implementation is different, particularly in the choice of evolution variable. Fragmentation is performed by a cluster model.

The ARIADNE program also uses a parton shower algorithm based on the MLLA framework. However, the perturbative QCD cascade is formulated in terms of two-parton systems which form color dipoles. When a gluon is radiated from a dipole, the dipole is converted into two independent dipoles. The color dipole model in ARIADNE differs from the other QCD cascade models in that it in a natural way treats most QCD coherence effects by describing the gluon bremsstrahlung in terms of radiation from color dipoles between partons, rather than treating partons as independent emitters. ARIADNE is one of the "Lund family of Monte Carlo programs" and is not a complete event generator. It does not provide fragmentation and decay processes. Instead, it is interfaced to the JETSET and PYTHIA fragmentation and decay routines. In addition, ARIADNE uses the JETSET or PYTHIA routines to generate the initial $q \bar{q}$ system and initial-state radiation.

\subsection{Bose-Einstein Correlations in Monte Carlo Generators}

BEC are implemented in MC models by a 'local reshuffling' method. The 'global reweighting' [67-70] and 'symmetrizing' methods [71] are not used since there is no MC successfully implementing them.

In the local reshuffling method [72], the momenta of particles are shuffled so that the $Q$ distribution is enhanced by either a Gaussian form $1+\lambda \exp \left(-R^{2} Q^{2}\right)$, or an exponential form $1+\lambda \exp (-R Q)$. This simulation is performed by the subroutine LUBOEI in the event generator JETSET [59] and the model is called $\mathrm{BE}_{0}$. The disadvantage of this model is that it has no quantum mechanical basis and energy-momentum conservation is (locally) violated. Also the naive assumption of a spherically symmetric Gaussian or exponential distribution is made for the source region in momentum space. Compensating shifts involving other pairs of particles are introduced to restore energy-momentum conservation. Several improved algorithms were developped, which 
shift the momenta in different ways: $\mathrm{BE}_{3}, \mathrm{BE}_{32}, \mathrm{BE}_{\mathrm{m}}, \mathrm{BE}_{\mathrm{m}}^{\mathrm{L}}$ and $\mathrm{BE}_{\lambda}$ [72]. They are incorporated, in addition to $\mathrm{BE}_{0}$, in the subroutine PYBOEI of PYTHIA.

\subsection{Tuning of Monte Carlo Parameters}

The Monte Carlo models involve several parameters. The distributions of particular event-shape variables are sensitive to certain parameters. The event-shape variables, such as the jet resolution parameter $\left(y_{23}\right)$, the charged-particle multiplicities $\left(n_{\mathrm{ch}}\right)$, etc, are used as tuning variables for the comparison of data and Monte Carlo.

The distribution of the four-momentum difference for like-sign and unlike-sign charged-particle pairs are also used when a model is tuned to describe BEC.

For a set of parameters, $\alpha$, to be tuned, the Monte Carlo distributions of the tuning variables are compared to the data distribution. This is quantified as

$$
\chi^{2}(\alpha)=\sum_{i=\text { tun.var }} \sum_{j=\text { bins }} \frac{[\operatorname{Data}(i, j)-\operatorname{MC}(i, j, \alpha)]^{2}}{\left[\sigma_{\text {Data }}^{\text {stat }}(i, j)\right]^{2}+\left[\sigma_{\text {Data }}^{\text {syst }}(i, j)\right]^{2}+\left[\sigma_{\text {MC }}^{\text {stat }}(i, j, \alpha)\right]^{2}}
$$

where the contributions to $\chi^{2}$ are summed over all bins $(j)$ of the distributions of the tuning variables $(i)$. The optimal parameter set is the one which minimises the above $\chi^{2}$. It is found using the CERN program package MINUIT [73]. More detailed information on tuning can be found in [74].

In the analysis of this thesis, $\mathrm{MC}$ samples are produced with or without $\mathrm{BE}_{0}$ for different purposes. For the detector correction of the correlation function, JETSET with $\mathrm{BE}_{0}$ on and off are used at generator level and at detector level. JETSET with and without $\mathrm{BE}_{0}$ are both tuned to L3 data [74]. When using other MC without BEC as reference samples, the samples are produced using a generator tuned [74] with $\mathrm{BE}_{32}$ on but used with $\mathrm{BE}_{32}$ off. In the comparison of 2- and 3jet events, all four MC's without BEC are used as reference samples. In HERWIG and ARIADNE it is difficult to trace back whether a given particle originates from a b-quark or not. This makes it impossible to select a gluon jet by anti-b tagging. Therefore, in the comparison of quark and gluon jets as well as in the comparison of the same side and different sides of the gluon jets, only JETSET and PYTHIA are used without BEC as reference samples. 


\section{Chapter 5}

\section{Comparison of BEC in 2-jet and 3-jet Events}

In this chapter, BEC in light-quark 2- and 3-jet events are compared. The Durham jet clustering algorithm is used with various values of $y_{\text {cut }}$ to classify the events as 2- or 3-jet events (3-jet events means events with more than 2 jets in this chapter). The BEC function $R_{2}$ is calculated, using different kinds of reference sample: mixed events, JETSET, PYTHIA, HERWIG and ARIADNE, all without BEC. Special attention is paid to the mixing procedure applied to build the reference sample needed for the construction of the BEC function and also to the unfolding of the data for detector effects. Finally, the parametrization of $R_{2}$ is performed and parameters $\lambda$ and $R$ are compared in the 2- and 3-jet events.

\subsection{Mixed Reference Sample}

In both light-quark 2-jet and 3-jet events, the BEC function $R_{2}$ is calculated using Eq. (1.25).

Two kinds of reference sample are used in this chapter: MC without BEC and mixed events. Mixed events are formed by mixing particles from different data events in the following way. Firstly, all events are rotated to a system with the $z$-axis along the thrust axis, the positive direction of which is chosen randomly, and the first 60,000 events are stored in a 'pool'. Then, the mixing process starts with the first event. For each particle in this event, a suitable substitute particle is randomly chosen from the events in the pool requiring:

1. the two particles are from two events which have the same multiplicity class (i.e., the same within 20\%),

2. the polar angle difference between the two particles is less than $5^{\circ}$,

3. the two particles have the same charge.

Once a particle from the pool is selected to replace one particle in the first event, the corresponding 'pool' event is removed from the pool and a following event is read into the pool. Thus 
all particles in the mixed event come from different events. This process continues until all events are mixed. When no more events are available to be used for the replacement of the 'pool' events, the replacement procedure starts over from the beginning of the event sample.

As a check on the method, several distributions before and after the event mixing are compared for 2-jet and 3-jet events, respectively, in Figs. 5.1 and 5.2. Good agreement of these distributions is found.

\subsection{Detector Correction}

There are several effects which must be unfolded from the two-particle correlation function. This is accomplished by two correction factors:

$$
R_{2}=\frac{\rho_{\text {data }}}{\rho_{\text {data,mix }}} \times C_{\text {det }} \times \frac{1}{C_{\text {mix }}} .
$$

The first correction factor, $C_{\text {det }}$, corrects for detector effects such as resolution, acceptance, efficiency, particle identification, while the second one, $C_{\mathrm{mix}}$, corrects for removing all correlations, not just BEC, by the mixing procedure.

Since the L3 detector does not identify hadrons, the detector correction factor, $C_{\text {det }}$, is estimated by a ratio of the two-pion correlation function from JETSET at the generator level to the two-particle correlation function using all particles from JETSET after full detector simulation, reconstruction and selection:

$$
C_{\mathrm{det}}=\frac{\left(\frac{\rho_{\mathrm{gen}}}{\rho_{\mathrm{gen}, \mathrm{mix}}}\right)_{\mathrm{pion}}}{\left(\frac{\rho_{\mathrm{det}}}{\rho_{\mathrm{det}, \mathrm{mix}}}\right)_{\mathrm{all}}} .
$$

The detector correction factor $C_{\text {det }}$ is estimated using JETSET either with or without BEC. In Fig. 5.3 the $C_{\text {det }}$ distribution is shown as a function of $Q$ for both cases, and both for 2-jet and 3 -jet events with $y_{\text {cut }}=0.005$. The ratio $C_{\mathrm{det}}^{2 \text {-jet }} / C_{\mathrm{det}}^{3 \text {-jet }}$ is also shown. It is clear that the detector correction term gives a large contribution to $R_{2}$. The value of $C_{\mathrm{det}}$, found using JETSET with BEC and without BEC, differ from each other in the low $Q$ region. This of course influences the BEC function and the extracted parameters $\lambda$ and $R$. However, as seen in Fig. 5.3(c), the influence is about the same on 2-jet events as on 3-jet events and thus is of little importance in the comparison of 2- and 3-jet events.

The second correction factor, $C_{\text {mix }}$, is estimated by $\mathrm{MC}$ at the generator level without BEC (JETSET, HERWIG, PYTHIA or ARIADNE):

$$
C_{\text {mix }}=\left(\frac{\rho_{\text {gen,noBE }}}{\rho_{\text {gen,noBE,mix }}}\right)_{\text {pion }} .
$$

Fig. 5.4 shows the $C_{\text {mix }}$ distribution as a function of $Q$ using different MC's without BEC at the generator level. It shows that $C_{\mathrm{mix}}$ is not MC dependent. JETSET no BEC is used to calculate $C_{\operatorname{mix}}$ in the following analysis. 

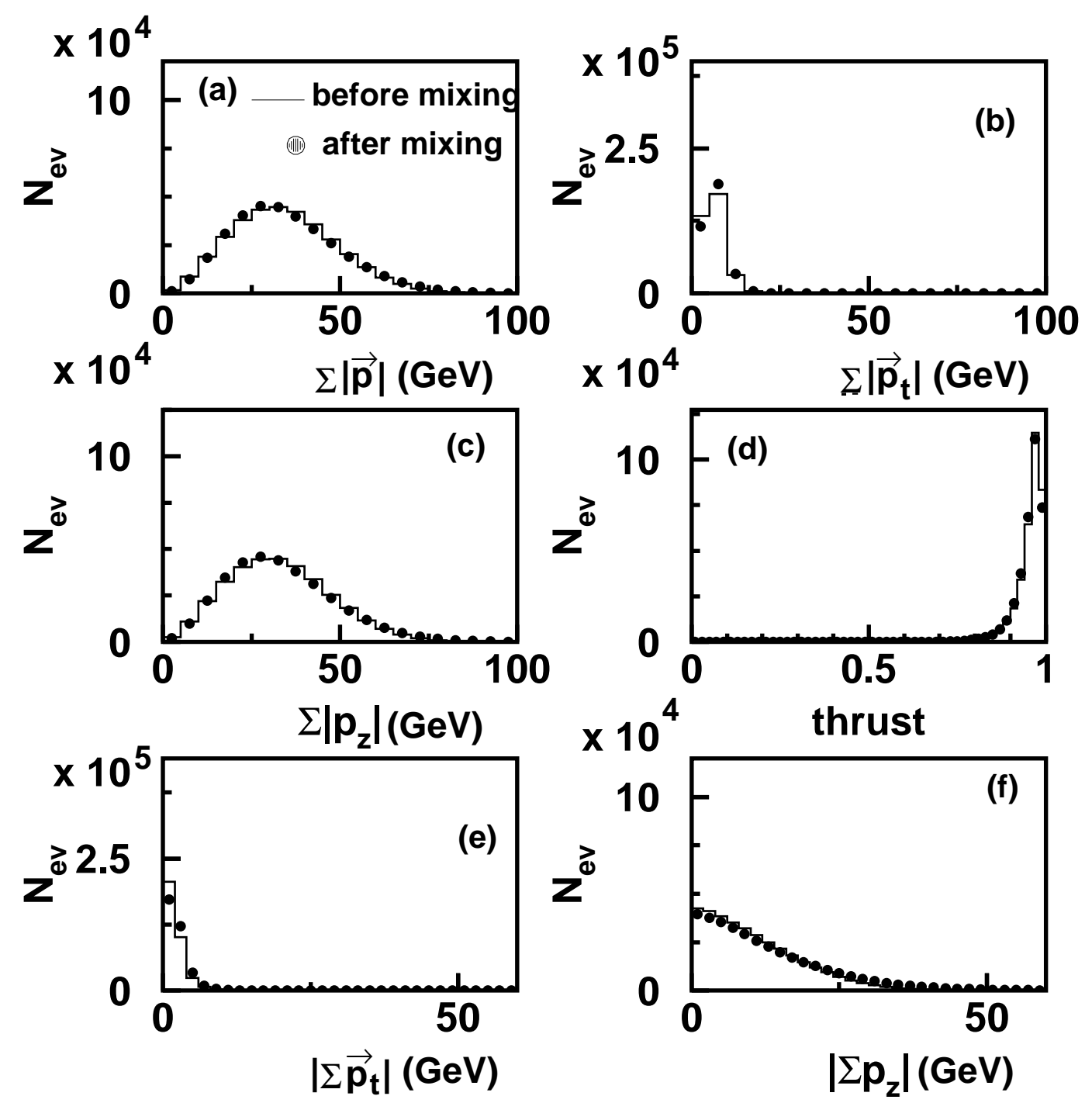

Figure 5.1: Distribution of various event quantities for the light-quark 2-jet $\left(y_{\text {cut }}=0.005\right)$ events before (histograms) and after mixing (dots) in the thrust frame using the raw data sample. Only charged particles are used. (a) sum of momenta $\sum|\vec{p}|$, (b) sum of transverse momenta $\sum\left|\vec{p}_{\mathrm{t}}\right|$, (c) sum of longitudinal momenta $\sum\left|p_{\mathrm{z}}\right|$, (d) thrust, (e) transverse momentum imbalance $\left|\sum \vec{p}_{\mathrm{t}}\right|$, (f) longitudinal momentum imbalance $\left|\sum p_{\mathrm{Z}}\right|$. 

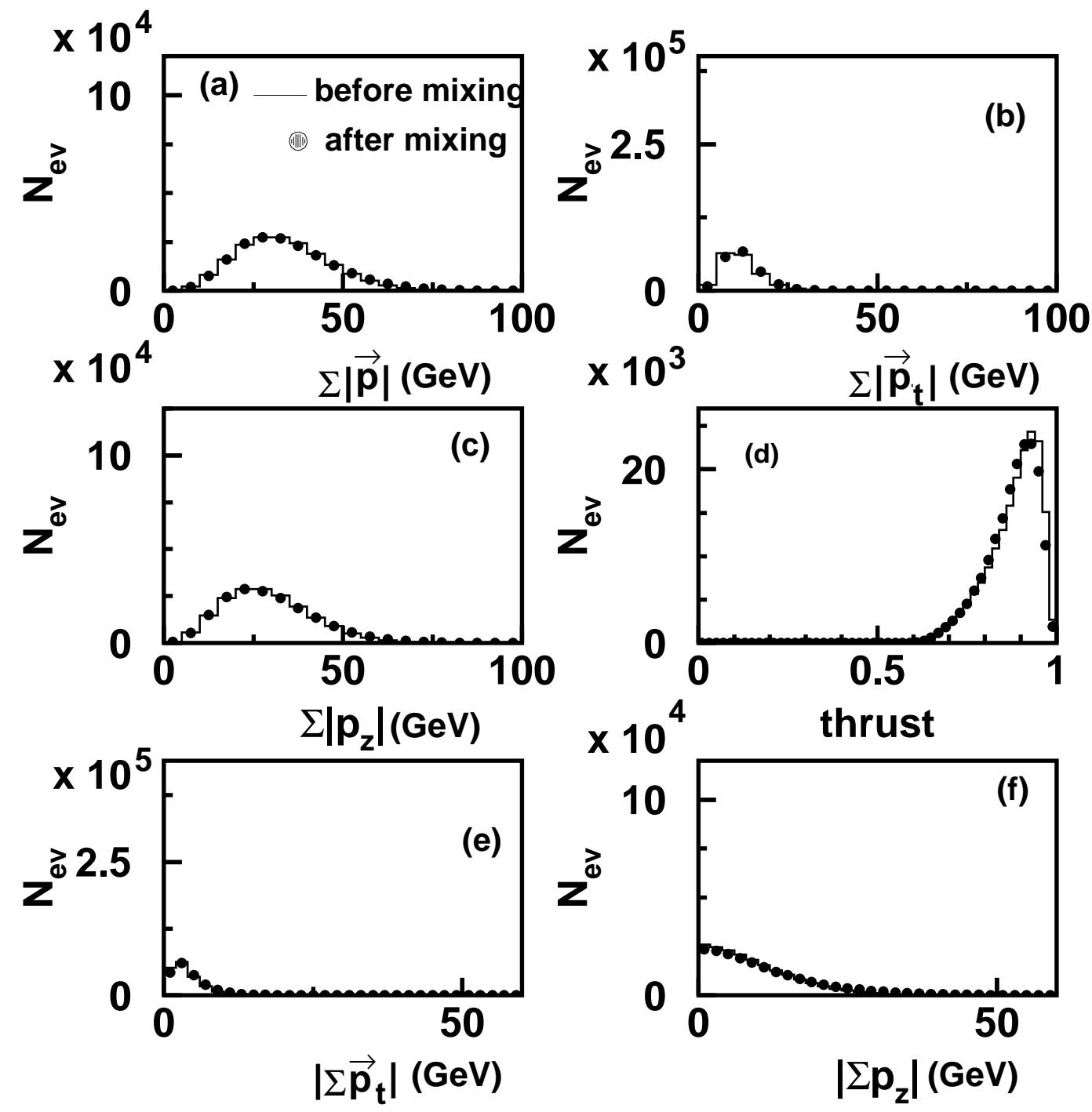

Figure 5.2: Distribution of various event quantities for the light-quark 3-jet events $\left(y_{\text {cut }}=0.005\right)$ before (histograms) and after mixing (dots) in the thrust frame using the raw data sample. Only charged particles are used. (a) sum of momenta $\sum|\vec{p}|$, (b) sum of transverse momenta $\sum\left|\vec{p}_{\mathrm{t}}\right|$, (c) sum of longitudinal momenta $\sum\left|p_{\mathrm{z}}\right|$, (d) thrust, (e) transverse momentum imbalance $\left|\sum \vec{p}_{\mathrm{t}}\right|$, (f) longitudinal momentum imbalance $\left|\sum p_{z}\right|$. 

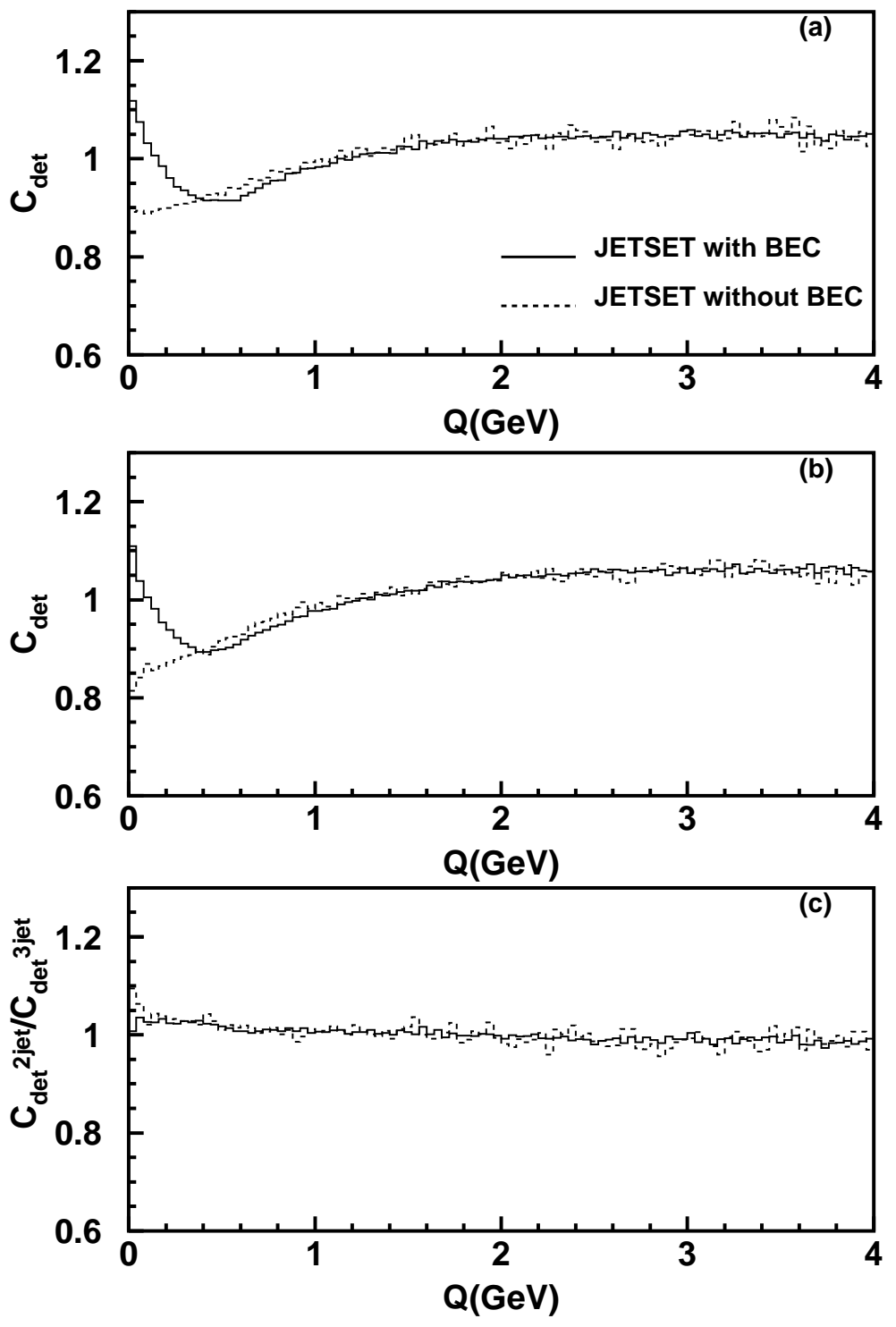

Figure 5.3: The detector correction factor $C_{\mathrm{det}}$ as a function of $Q$ in the light-quark 2-jet events (a), and in the light-quark 3-jet events (b) using JETSET with BEC (full histograms) and without BEC (dashed histograms). The ratio of $C_{\mathrm{det}}$ for 2 -jet events to $C_{\mathrm{det}}$ for 3 -jet events is shown in (c). The Durham algorithm with $y_{\text {cut }}=0.005$ is used to select 2-jet and 3-jet events. 

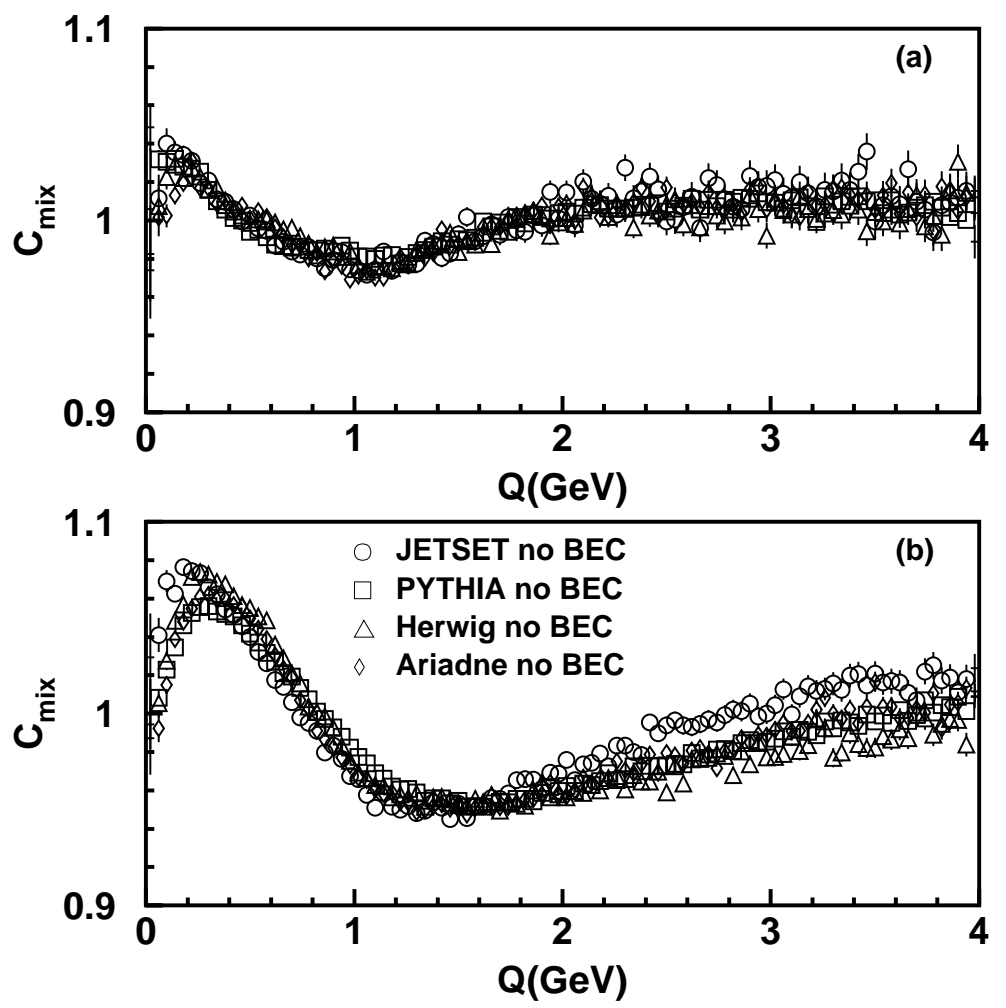

Figure 5.4: The $C_{\mathrm{mix}}$ distribution as a function of $Q$ in the light-quark 2-jet events (a), and in the light-quark 3-jet events (b) using four MC generators JETSET, PYTHIA, HERWIG and ARIADNE without BEC. The Durham algorithm with $y_{\text {cut }}=0.005$ is used to select 2-jet and 3-jet events. 
Instead of mixed events, a Monte Carlo without BEC can be used as a reference sample. With $C_{\text {det }}$ given by $\frac{\left(\rho_{\text {gen }}\right)_{\text {pion }}}{\left(\rho_{\text {det }}\right)_{\text {all }}}, R_{2}$ then becomes

$$
R_{2}=\frac{\rho_{\text {data }} \times \frac{\left(\rho_{\text {gen }}\right)_{\text {pion }}}{\left(\rho_{\text {det }}\right)_{\text {all }}}}{\left(\rho_{\text {gen }, \text { noBE }}\right)_{\text {pion }}},
$$

where $\left(\rho_{\text {gen,noBE}}\right)_{\text {pion }}$ is calculated using the four MC's, and $C_{\text {det }}$ is calculated using JETSET with and without BEC, respectively.

\subsection{Dependence of $\boldsymbol{R}_{\mathbf{2}}$ on Number of Jets and Multiplicity}

As an illustration, Fig. 5.5 shows the $R_{2}$ distribution for 2-jet and 3-jet light-quark events using different reference samples: mixed events, JETSET no BEC, PYTHIA no BEC, HERWIG no BEC and ARIADNE no BEC. Fits of the Edgeworth parametrization Eq. (1.27) in the range 0.04-2.80 $\mathrm{GeV}$ are shown as solid curves. It is seen that this parametrization provides an adequate description of $R_{2}$.

\subsubsection{Comparison of $\lambda$ and $R$ in 2- and 3-jet Events}

Fig. 5.6 shows the parameters $\lambda$ and $R$ for 2- and 3-jet events as a function of $y_{\text {cut }}$ using each of the reference samples. From the plot we see that $\lambda$ does not change significantly with increasing $y_{\text {cut }}$, while $R$ increases with increasing $y_{\text {cut }}$, especially in the 3 -jet events. We also notice that $\lambda$ and $R$ are reference-sample dependent. Using mixed events as the reference sample gives the smallest value for $\lambda$ and a medium value for $R$. Furthermore, by studying Fig. 5.6(c) and (f), i.e., $\lambda_{2 \text {-jet }}-\lambda_{3 \text {-jet }}$ and $R_{2 \text {-jet }}-R_{3 \text {-jet }}$, we find $\lambda$ to be slightly smaller in 3 -jet than in 2 -jet events, while $R$ is slightly bigger in 3 -jet than in 2 -jet events. Similar behavior has been previously observed by OPAL [75] and DELPHI [23].

As we have seen from the $C_{\text {det }}$ distribution (Fig. 5.3), there are some differences between the detector correction factors calculated from JETSET with and without BEC. The effect on the values of $\lambda$ and $R$ is seen by comparing Fig. 5.6, where $C_{\text {det }}$ is calculated using JETSET with BEC, with Fig. 5.7, where $C_{\text {det }}$ is calculated using JETSET without BEC. While the correlation strength $\lambda$ is obviously smaller when JETSET without BEC is used, the tendency of $\lambda$ and $R$ with $y_{\text {cut }}$ is similar. Studying the 2-jet and 3-jet difference on $\lambda$ and $R$, we see $\lambda_{2 \text {-jet }}-\lambda_{3 \text {-jet }}$ and $R_{2 \text {-jet }}-R_{3 \text {-jet }}$ spread in a wider range when using JETSET without BEC. This is also consistent with the result in Fig. 5.3(c). Considering the statistical errors, we conclude that there is no significant evidence for a difference in $\lambda$ or $R$ between 2-jet and 3-jet events or for a dependence of $\lambda$ or $R$ on the value of $y_{\text {cut }}$. This conclusion is independent of the choice of reference sample.

\subsubsection{Multiplicity Dependence of $\boldsymbol{\lambda}$ and $\boldsymbol{R}$ in 2 - and 3-jet Events}

The average charged-particle multiplicity $n_{\mathrm{ch}}$ is larger in 3-jet events than in 2-jet events. Fig. 5.8 shows the charged-particle multiplicity distribution for the data and charged-pion multiplicity dis- 

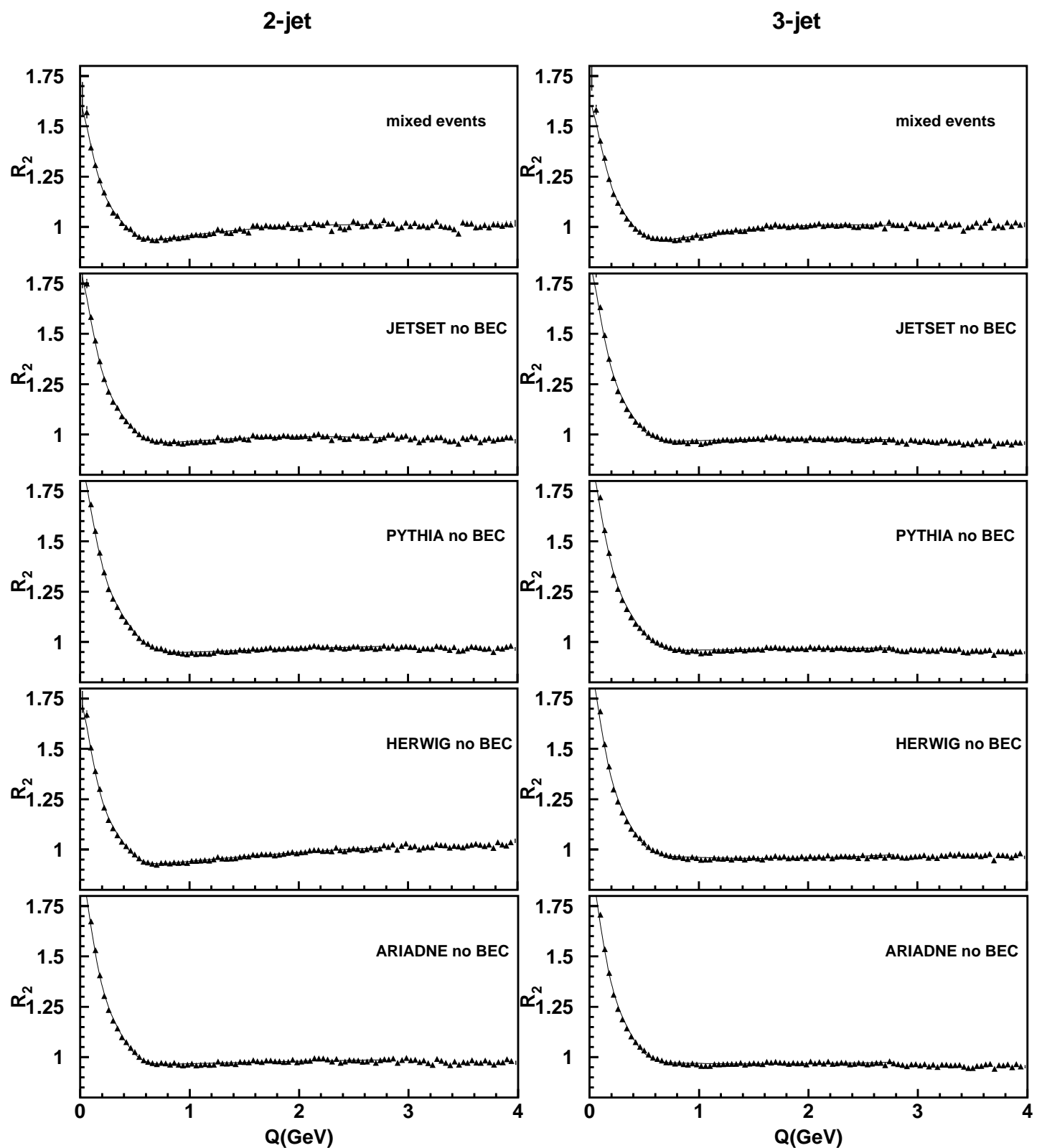

Figure 5.5: The $R_{2}$ distribution for 2-jet and 3-jet light-quark events using different reference samples: mixed events, JETSET no BEC, PYTHIA no BEC, HERWIG no BEC and ARIADNE no BEC. $C_{\text {det }}$ is calculated using JETSET with BEC. 2 -jet and 3-jet events are selected with $y_{\text {cut }}=$ 0.005. The Edgeworth fits Eq. (1.27) are shown as the solid curves (in the range 0-2.8 GeV). 
2-jet
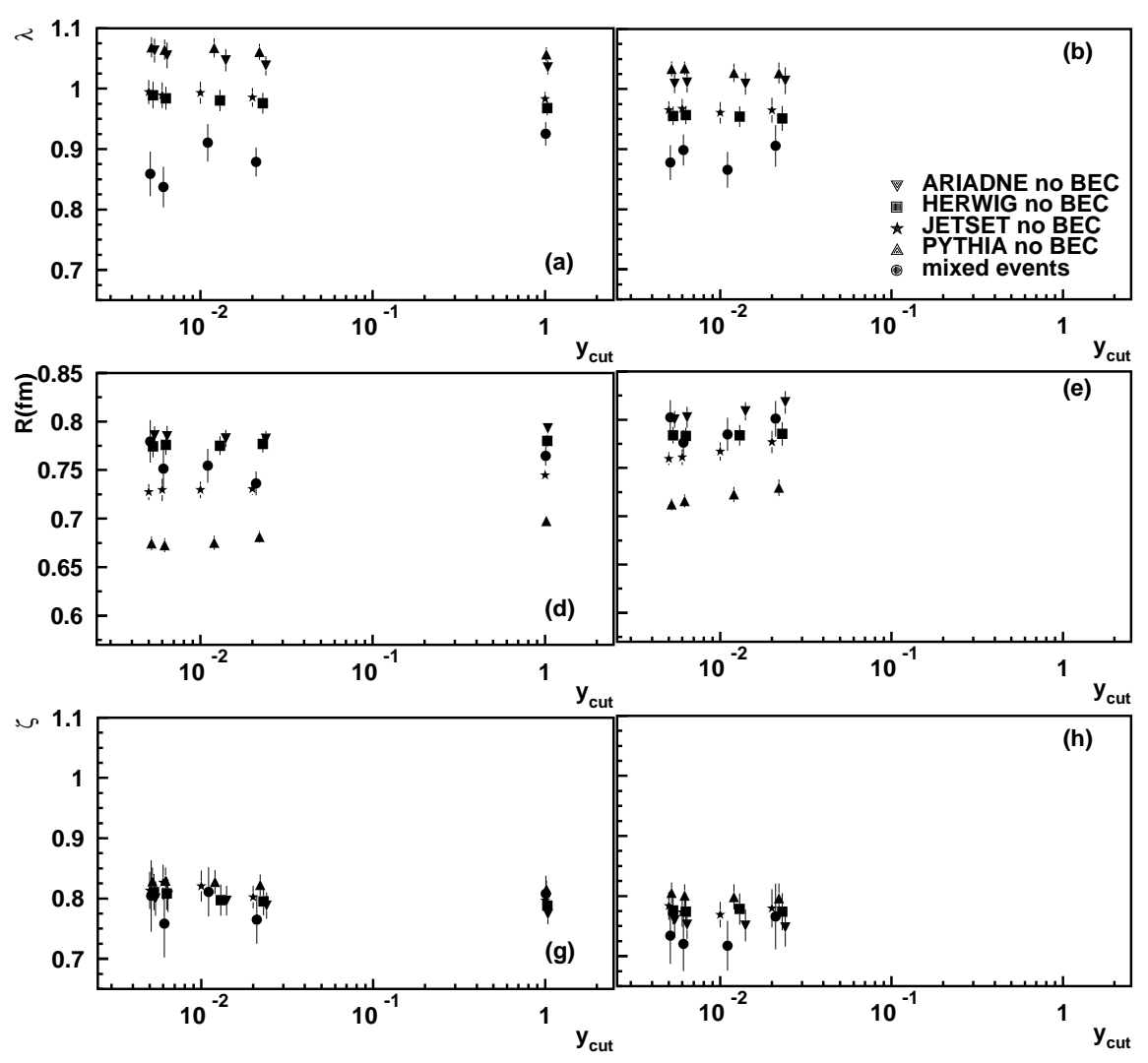

$\Delta$
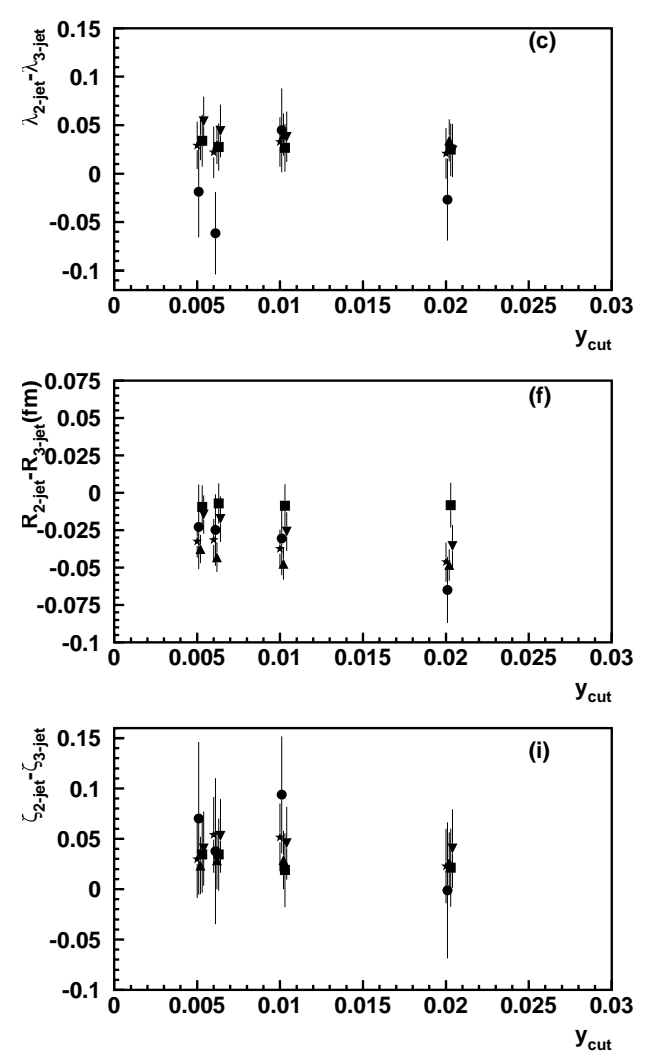

Figure 5.6: $\lambda$, (a) and (b), $R$, (d) and (e), and $\zeta$, (g) and (h) vs. $y_{\text {cut }}$ in 2-jet, (a), (d) and (g), and 3-jet, (b), (e) and (h) events. ARIADNE no BEC (down-triangles), HERWIG no BEC (squares), JETSET no BEC (stars), PYTHIA no BEC (up-triangles), and mixed events (dots) are used as reference sample. $C_{\text {det }}$ is calculated using JETSET with BEC. $\lambda_{2 \text {-jet }}-\lambda_{3 \text {-jet }}, R_{2 \text {-jet }}-R_{3 \text {-jet }}$ and $\zeta_{2 \text {-jet }}-\zeta_{3 \text {-jet }}$ are shown in (c), (f) and (i). Error bars are statistical only. For clarity, the points are slightly displaced in $y_{\text {cut }}$. 
2-jet
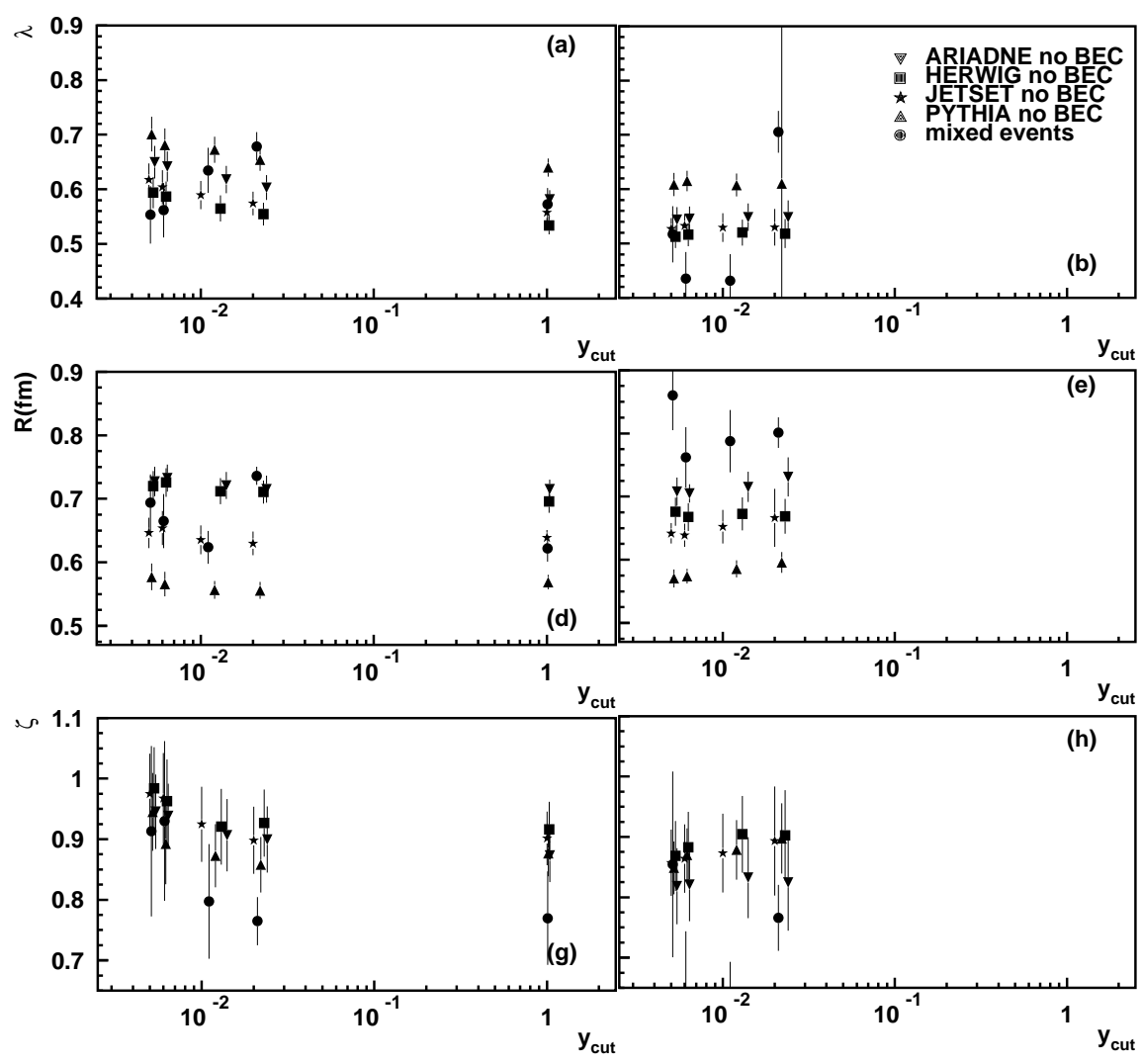

$\Delta$
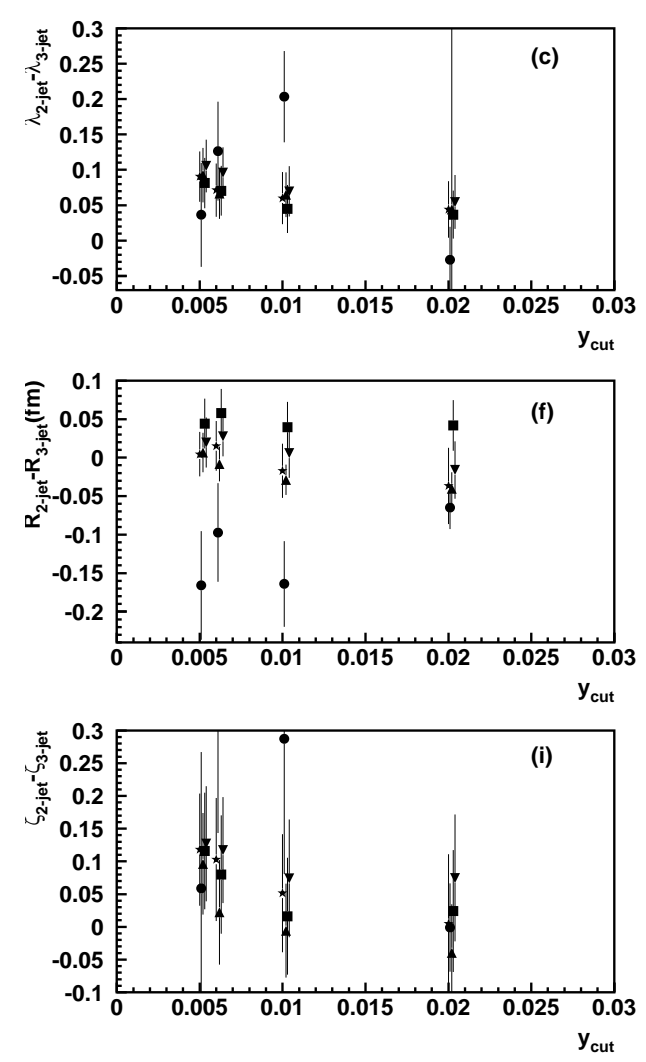

Figure 5.7: $\lambda$, (a) and (b), $R,(\mathrm{~d})$ and (e), and $\zeta$, (g) and (h) vs. $y_{\text {cut }}$ in 2-jet, (a), (d) and (g), and 3-jet, (b), (e) and (h) events. ARIADNE no BEC (down-triangles), HERWIG no BEC (squares), JETSET no BEC (stars), PYTHIA no BEC (up-triangles), and mixed events (dots) are used as reference sample. $C_{\text {det }}$ is calculated using JETSET without BEC. $\lambda_{2 \text {-jet }}-\lambda_{3 \text {-jet }}, R_{2 \text {-jet }}-R_{3 \text {-jet }}$ and $\zeta_{2 \text {-jet }}-\zeta_{3 \text {-jet }}$ are shown in (c), (f) and (i). Error bars are statistical only. For clarity, the points are slightly displaced in $y_{\text {cut }}$. 


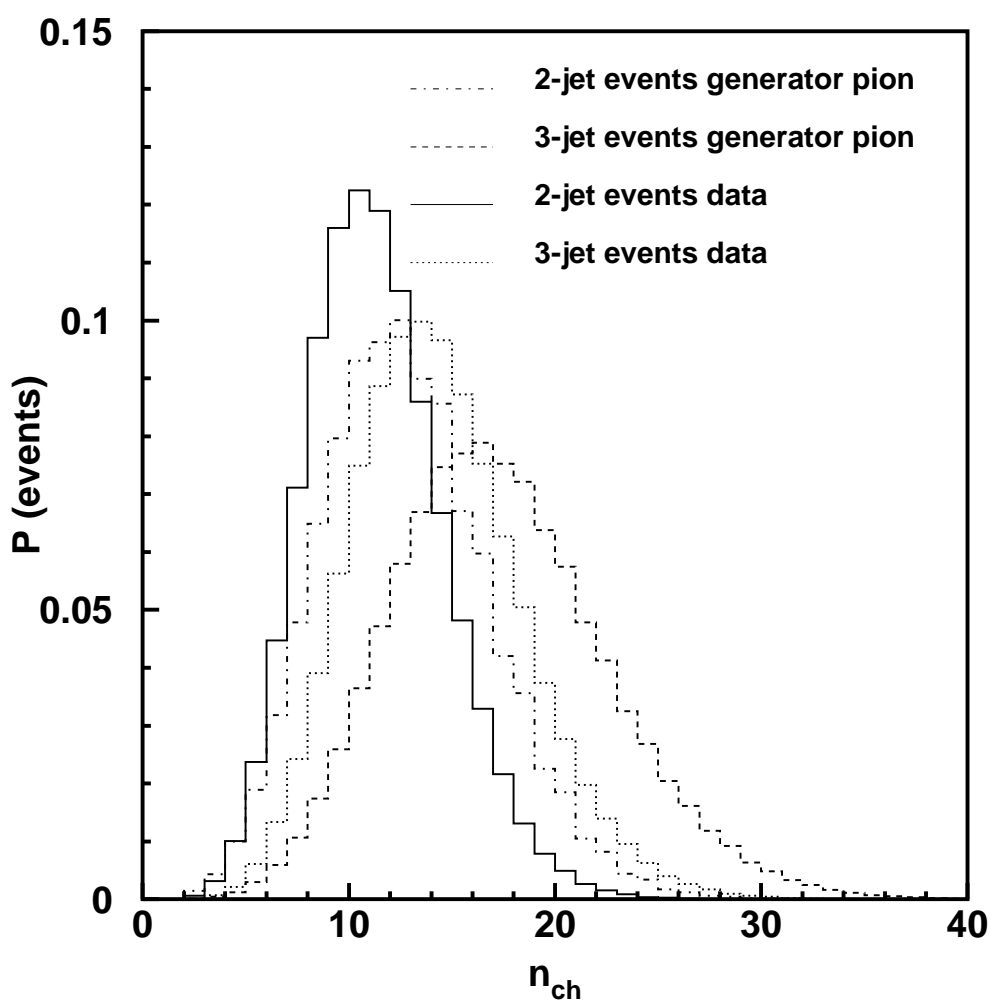

Figure 5.8: The charged-particle multiplicity distribution in the light-quark 2- (solid line) and 3-jet (dotted line) events for the data, and the charged-pion multiplicity distribution in the light-quark 2(dashdotted line) and 3-jet (dashed line) events at generator level (JETSET) with $y_{\text {cut }}=0.005$.

tribution at the generator level in the light-quark 2- and 3-jet events. To remove a possible multiplicity effect on $\lambda$ and $R$ in 2 - and 3-jet events, $\lambda$ and $R$ are studied in different multiplicity regions. Since no significant $y_{\text {cut }}$ dependence is seen in Fig. 5.6 and Fig. 5.7, this multiplicity-dependent analysis is limited to a single value of $y_{\text {cut }}$, namely 0.005 .

Based on the multiplicity distribution of charged particles and charged pions, five multiplicity intervals are chosen for each distribution where each interval contains approximately $20 \%$ of the number of events. The five intervals for data and $\mathrm{MC}$ detector level in light-quark 2-jet events are: 3-9, 10-11, 12-13, 14-15, 16-26, and in light-quark 3-jet events: 3-11, 12-13, 14-15, 16-18, 19-30. The five intervals for generator level in light-quark 2-jet events are: 2-9, 10-11, 12-13, 14-15, 16-30, and in light-quark 3-jet events: 2-13, 14-16, 17-19, 20-23, 24-40.

In each interval, $R_{2}$ is calculated and fitted by the Edgeworth expansion. $R_{2}$ is calculated using corresponding multiplicity intervals of data and generator level, e.g., the first data interval for data and the detector level MC and the first generator level interval for generator level MC. Fig. 5.9 shows the resulting values of $\lambda, R$ and $\zeta$ as a function of $n_{\mathrm{ch}}$ (i.e., the five $n_{\mathrm{ch}}$ intervals at generator level, where horizontal error bars indicate the range of intervals and points are centered at the mean multiplicity of each interval) for the 2-jet and 3-jet events. Here mixed events are used as the reference sample and $C_{\text {det }}$ is calculated using JETSET with BEC. Fig. 5.10 is the same as Fig. 5.9, except that $C_{\text {det }}$ is calculated using JETSET without BEC. Both $\lambda$ and $R$ are shifted to smaller 
values compared with Fig.5.9.

Both Fig. 5.9 and Fig. 5.10 lead to the conclusion that $\lambda$ does not show an obvious $n_{\mathrm{ch}}$ dependence, while $R$ increases with increasing $n_{\mathrm{ch}}$. Further, for a given $n_{\mathrm{ch}}$, the values of $\lambda$ and $R$ are consistent for 2-jet and 3-jet events.

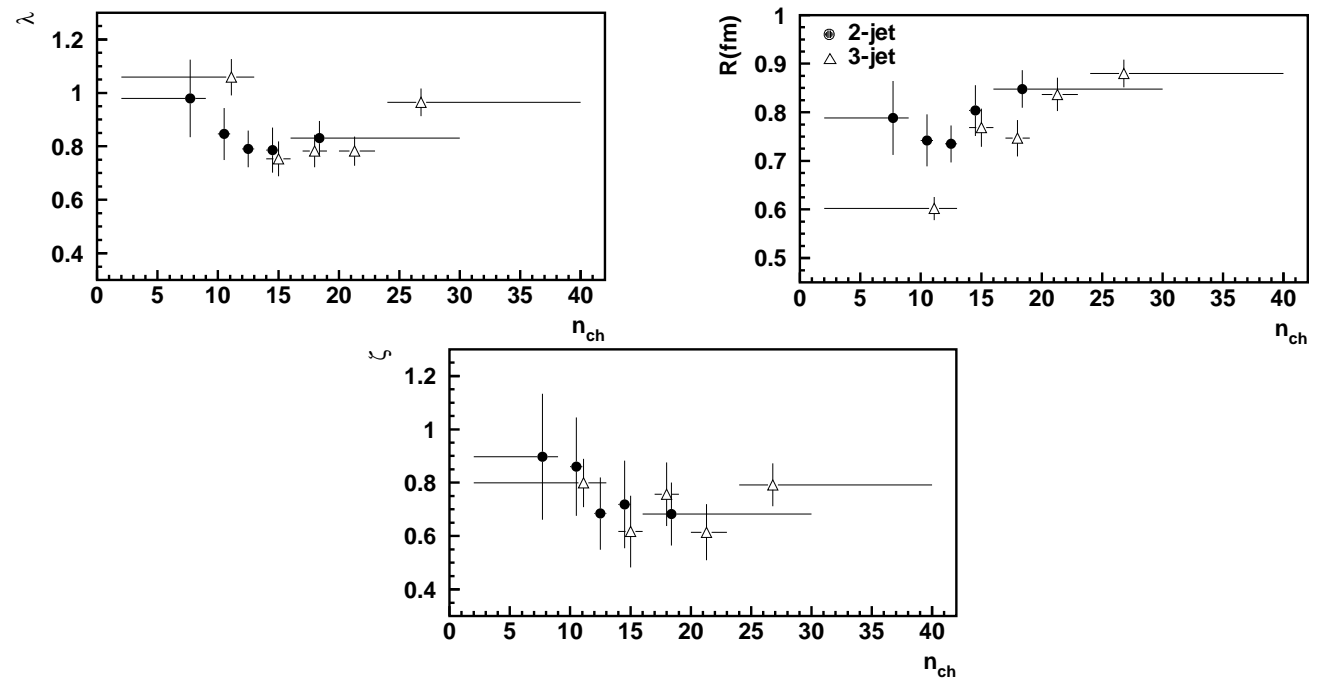

Figure 5.9: $\lambda$ (upper-left), $R$ (upper-right) and $\zeta$ (below) vs. $n_{\mathrm{ch}}$ in the 2 -jet (dots) and the 3 -jet (triangles) events. Mixed events are used as the reference sample. $C_{\text {det }}$ is calculated using JETSET with BEC. The value of $n_{\mathrm{ch}}$ for each point means the mean multiplicity in the interval at generator level. Horizontal error bars indicate the ranges of the 5 intervals. Vertical error bars represent the statistical errors only.

Fig. 5.11 shows $\lambda, R$ and $\zeta$ as a function of $n_{\mathrm{ch}}$ (i.e., the five $n_{\mathrm{ch}}$ intervals at generator level) in the 2-jet and the 3-jet events when MC's without BEC are used as reference samples. PYTHIA no BEC, HERWIG no BEC and ARIADNE no BEC are used as the reference samples. $C_{\text {det }}$ is calculated using JETSET with BEC. $\lambda$ shows no obvious $n_{\mathrm{ch}}$ dependence, while $R$ shows some. No obvious difference between 2-jet and 3-jet events is observed for both $\lambda$ and $R$. This is consistent with the conclusion reached by using mixed events as reference sample.

With a simple Gaussian parametrization and using pairs of unlike-charged particles as a reference sample, OPAL [75] found an $R$ value approximately 6-10\% larger for three-jet events than for two-jet events, for all multiplicities. Considering the large uncertainties due to the use of parametrization and reference sample, we do not consider this a significant difference to our result. More importantly, our result on the similarity of the $\lambda$ values are consistent with the result of OPAL.

\subsection{Conclusion}

The correlation strength $\lambda$ and source radius $R$ are compared in 2-jet and 3-jet events for different values of the Durham resolution parameter $y_{\text {cut }}$. If we consider different reference samples and different detector corrections as part of systematic uncertainties, the conclusion can be drawn that 


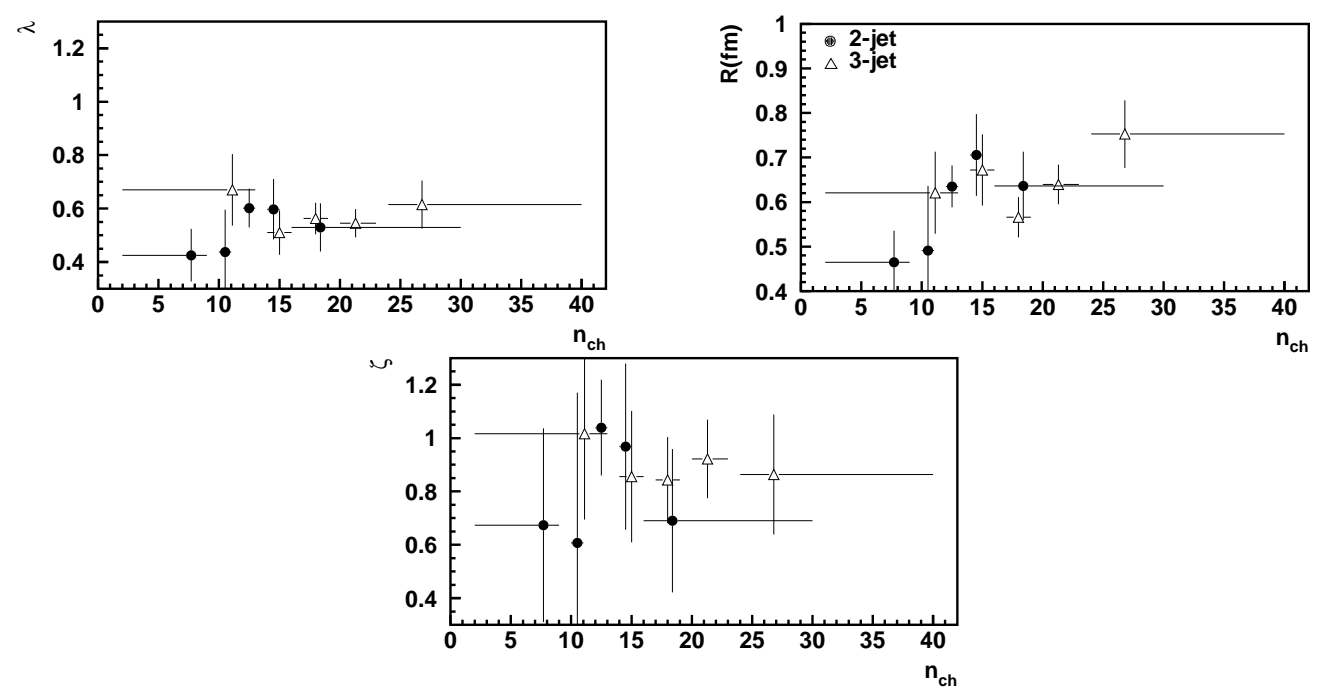

Figure 5.10: $\lambda$ (upper-left), $R$ (upper-right) and $\zeta$ (below) vs. $n_{\mathrm{ch}}$ in the 2 -jet (dots) and the 3 jet (triangles) events. Mixed events are used as the reference sample. $C_{\text {det }}$ is calculated using JETSET with no BEC. The value of $n_{\mathrm{ch}}$ for each point means the mean multiplicity in the interval at generator level. Horizontal error bars indicate the ranges of the 5 intervals. Vertical error bars represent the statistical errors only.

no $y_{\text {cut }}$ dependence is observed for $\lambda$ and $R ; \lambda$ and $R$ do not show any significant difference in 2-jet and in 3-jet events, independent of whether all multiplicities or restricted multiplicity intervals are used. 

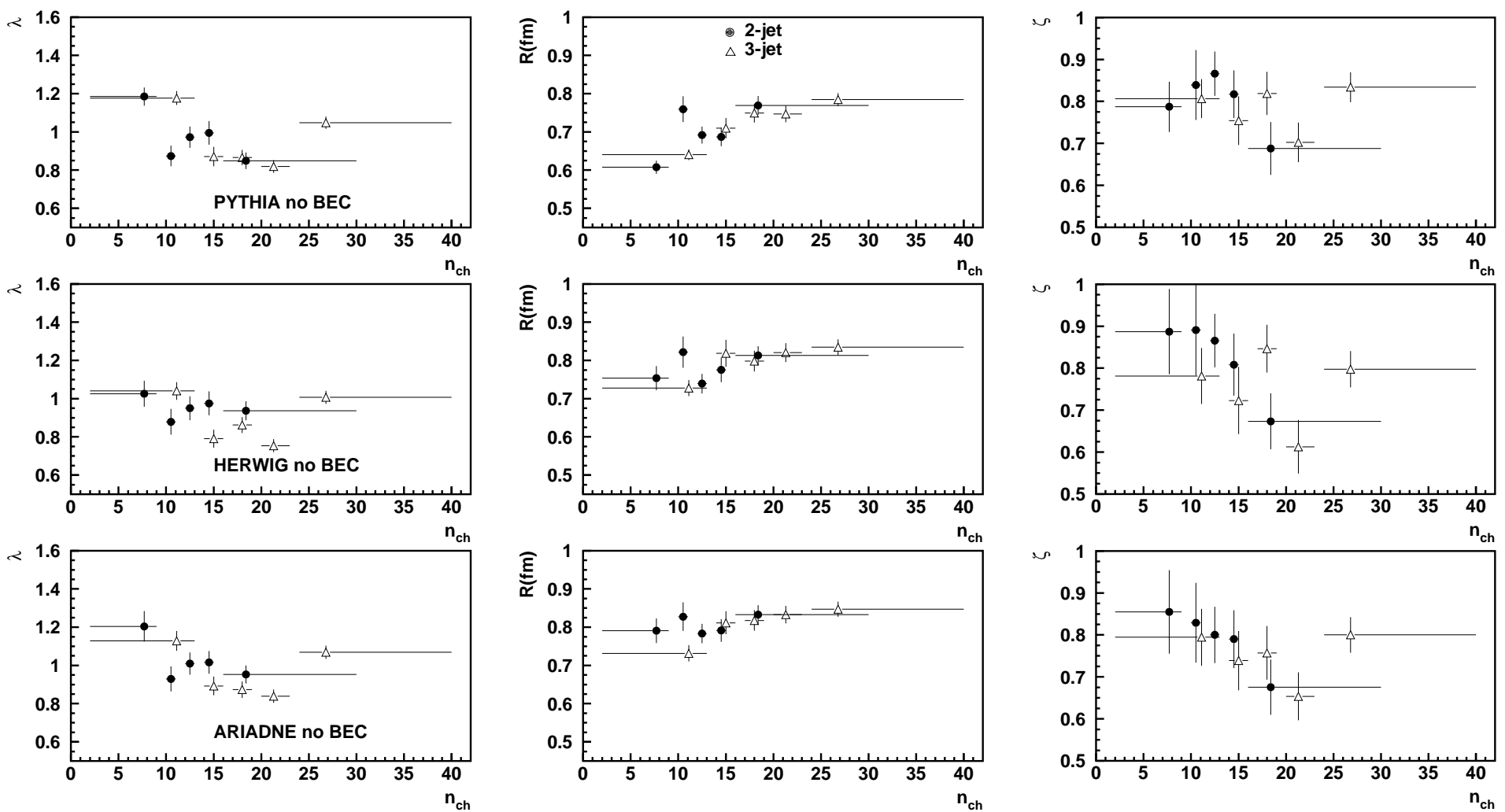

Figure 5.11: $\lambda$ (left), $R$ (middle) and $\zeta$ (right) vs. $n_{\mathrm{ch}}$ in the 2 -jet (dots) and the 3 -jet (triangles) events. PYTHIA no BEC, HERWIG no BEC and ARIADNE no BEC are used as the reference sample. $C_{\mathrm{det}}$ is calculated using JETSET with BEC. The value of $n_{\mathrm{ch}}$ for each point means the mean multiplicity in the interval at generator level. Vertical error bars represent the statistical errors only. 


\section{Chapter 6}

\section{Comparison of Quark and Gluon Jets}

In the previous chapter, we found no significant difference in the values of $\lambda$ and $R$ between the 2 -jet and 3-jet samples. This may be due to incomplete overlap of the two strings in the 3-jet events. The overlap is largest in the gluon jet and smallest in the quark jets. Possible signals of $\lambda$ suppression and $R$ increase in the 3 -jet events can be buried by particles coming from two non-overlapping strings. The difference between quark and gluon jets is, therefore, expected to be more sensitive to inter-string BEC than the difference between 2- and 3-jet events.

In this chapter, a comparison of quark and gluon jets is presented. Quark and gluon jets are selected using the method described in Sect. 3.7. Firstly, a comparison of quark and gluon jets with the same energy is performed. Then, $\lambda$ and $R$ are compared in quark and gluon jets with consideration of hardness, rapidity and scaled momentum, $x$, dependence.

\subsection{Hardness Dependence of $\lambda$ and $R$ in Quark and Gluon Jets}

The gluon jets have, on average, lower energy than the quark jets. Before comparing quark and gluon jets, we check whether the results on $\lambda$ and $R$ really reflect the difference of quark and gluon jets, or merely the energy difference. Instead of using jet energy, we use jet hardness defined as

$$
h=E_{\text {jet }} \sin \left(\frac{\theta_{1,2}}{2}\right),
$$

where $E_{\text {jet }}$ is the energy of the jet and $\theta_{1,2}$ is the smaller of the two angles between this jet and the other two jets. Hardness is equal to the jet energy for 2-jet events.

Fig. 6.1 shows the jet hardness distribution for gluon jets (the least energetic jet in the lightquark events and the jet after performing double b-tagging in the b-quark events), second-most energetic quark jets and most energetic quark jets in the light-quark events, and quark jets in the b-quark events. There is reasonably good agreement between the distributions for the light- and b-quark events.

Three hardness ranges are chosen for the gluon jets $(6-9,9-14.5$ and $14.5-34 \mathrm{GeV})$ and four for the quark jets (10-26 and 30-46 GeV for the two quark jets in the light-quark 3-jet events, 35-48 and 45-58 GeV for the two quark jets in the light-quark 2-jet events). $R_{2}$, Eq. (5.4), is 


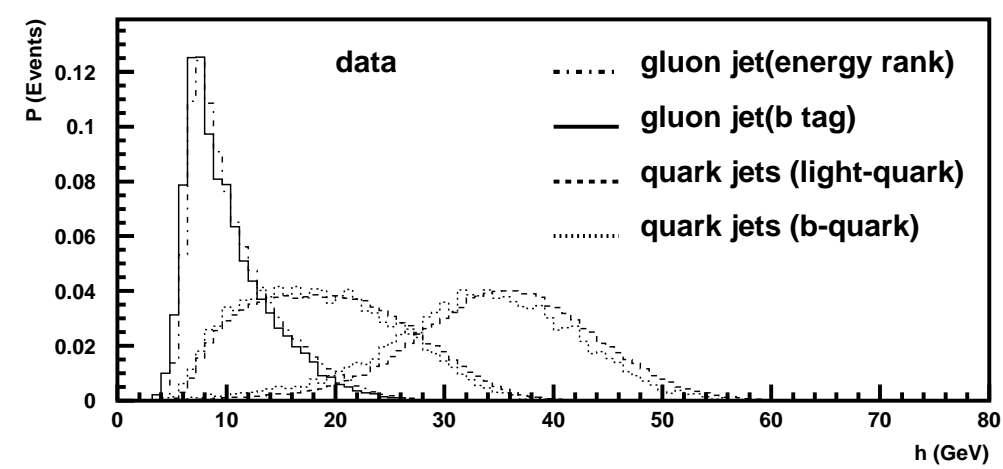

Figure 6.1: Hardness distribution for gluon jets (dashdotted line using energy rank and solid line using double b-tagging), and quark jets (dashed line for the light-quark events and dotted line for the b-quark events) in the data sample.

calculated using Monte Carlo (JETSET and PYTHIA) without BEC as the reference sample, and parametrized using the Edgeworth expansion Eq. (1.27) with parameters $\delta=\varepsilon=0$.

The values of $\lambda$ and $R$ are influenced by the choice of Monte Carlo for determining $C_{\mathrm{det}}$ and for the reference sample.

Fig. 6.2 shows $C_{\text {det }}$ as a function of $Q$ for quark and gluon jet samples using JETSET with and without BEC. The ratio $C_{\mathrm{det}}^{\text {quark }} / C_{\mathrm{det}}^{\text {gluon }}$ is also shown. Here, the gluon jet is selected using energy rank in the light-quark 3-jet events, and the quark jet is the most energetic jet in the light-quark 3 -jet events. The second highest energetic jet is not used. Fig. 6.3 also shows the $C_{\text {det }}$ distribution and the ratio $C_{\mathrm{det}}^{\text {quark }} / C_{\mathrm{det}}^{\text {gluon }}$. The only difference from Fig. 6.2 is that the gluon jet is selected by using double b-tagging in the b-quark events. From Figs. 6.2 and 6.3, it is clear that $C_{\text {det }}$ is different when using JETSET with BEC and without BEC. However, as seen in Figs. 6.2(c) and 6.3(c), the influence on quark and gluon jets is rather similar. Hence, the difference between $R_{2}$ for quark and gluon jets in low-Q area could not be caused by $C_{\mathrm{det}}$.

The fits of the Edgeworth parametrization to the data for the various hardness ranges are in general acceptable. Examples of the fits are shown in Fig. 6.4 and Table 6.1. The resulting values of the parameters $\lambda, R$ and $\zeta$ are shown in Fig. 6.5 for quark and gluon jets in the various hardness ranges. We use JETSET as reference sample in the left two plots and PYTHIA in the right two plots. $C_{\text {det }}$ is calculated using JETSET with BEC. No matter which reference sample we use, $\lambda$, $R$ and $\zeta$ do not have an obvious hardness dependence. Furthermore, $\lambda, R$ and $\zeta$ are the same for quark and gluon jets.

The results using JETSET without BEC to determine $C_{\text {det }}$ are shown in Fig. 6.6. No obvious hardness dependence is seen. However, $R$ in light-quark 2 -jet events is dramatically small. There is little difference in the values of $\lambda, R$ and $\zeta$ between quark and gluon jets.

The purity is shown in Fig. 6.7 as a function of the hardness of the gluon jet in b-quark and light-quark events. It is seen that this purity is smaller in light-quark events than in b-quark events. This could partly explain why $\lambda, R$ and $\zeta$ behave slightly differently for the gluon jet in light-quark and b-quark events. Smaller gluon purity means larger contamination from quark jets. Thus we expect the values of $\lambda, R$ and $\zeta$ for light-quark events to be closer to those for quark jets, which, as can be seen from Figs. 6.5 and 6.6 is usually the case. 

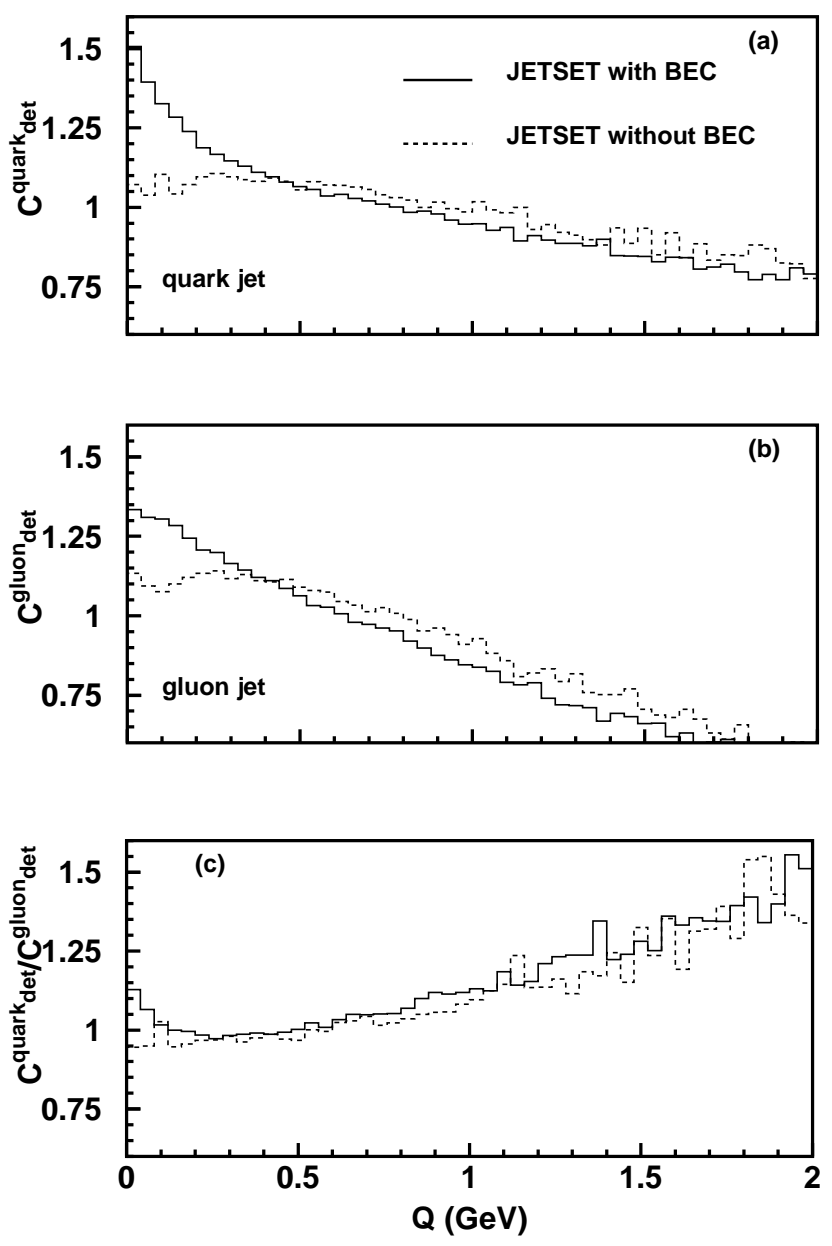

Figure 6.2: The $C_{\text {det }}$ distribution as a function of $Q$ in quark jets (a), and in gluon jets (b) using JETSET with BEC (full histograms) and without BEC (dashed histograms). The ratio of $C_{\text {det }}$ for quark jets to $C_{\text {det }}$ for gluon jets is shown in (c). Gluon jets are selected using energy rank in the light-quark 3-jet events, and quark jets are the most energetic jets in the light-quark 3-jet events.

\begin{tabular}{|c|c|c|c|c|}
\hline samples & $\lambda$ & $R(\mathrm{fm})$ & $\zeta$ & $\chi^{2} / \mathrm{NDOF}$ \\
\hline gluon in light-quark events & $0.75 \pm 0.13$ & $0.93 \pm 0.10$ & $0.93 \pm 0.24$ & $44 / 44$ \\
\hline gluon in heavy-quark events & $0.31 \pm 0.28$ & $0.48 \pm 0.16$ & $-0.01 \pm 2.24$ & $45 / 44$ \\
\hline
\end{tabular}

Table 6.1: The values of the fitted parameters $\lambda, R$ and $\zeta$ as well as the $\chi^{2}$ and number of degrees of freedom for the fits shown in Fig. 6.4 

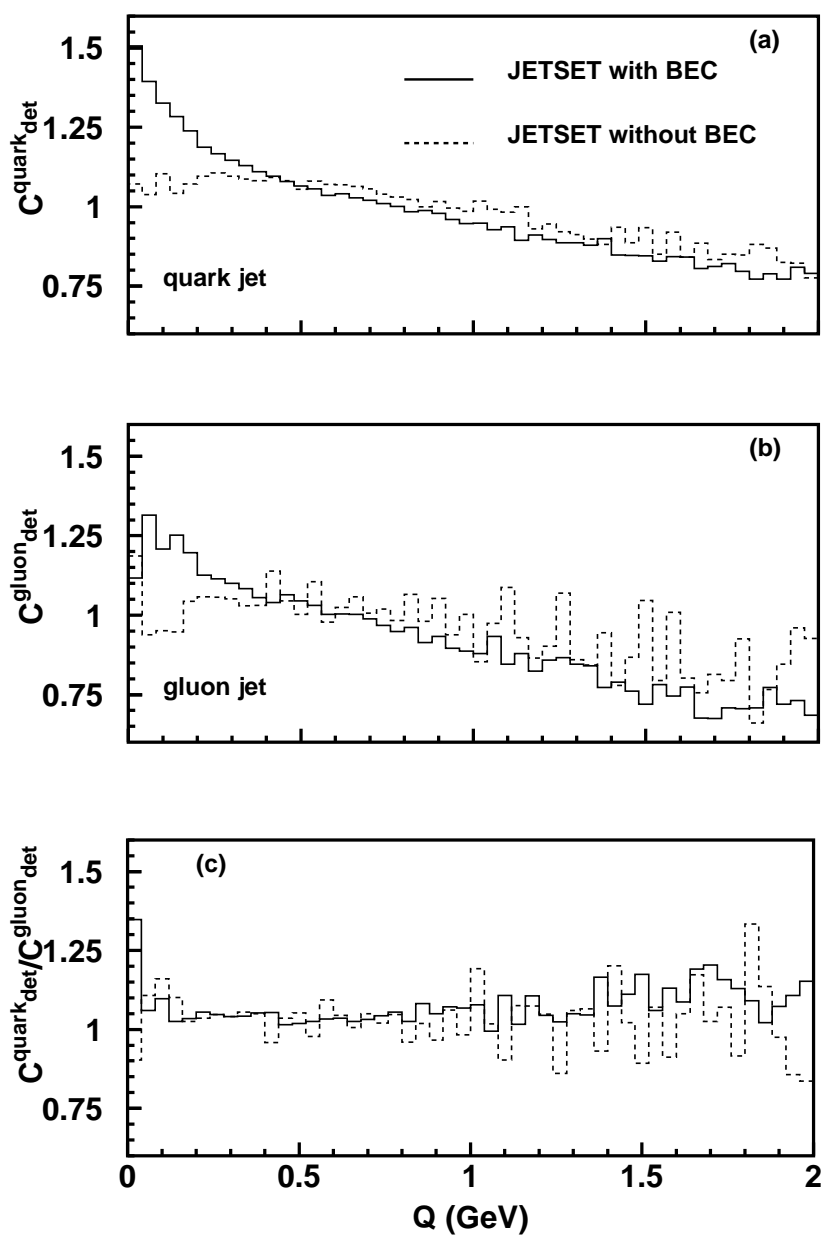

Figure 6.3: The $C_{\text {det }}$ distribution as a function of $Q$ in quark jets (a), and in gluon jets (b) using JETSET with BEC (full histograms) and without BEC (dashed histograms). The ratio of $C_{\mathrm{det}}$ for quark jets to $C_{\text {det }}$ for gluon jets is shown in (c). Gluon jets are selected using double b-tagging in the b-quark events, and quark jets are the most energetic jets in the light-quark 3-jet events.
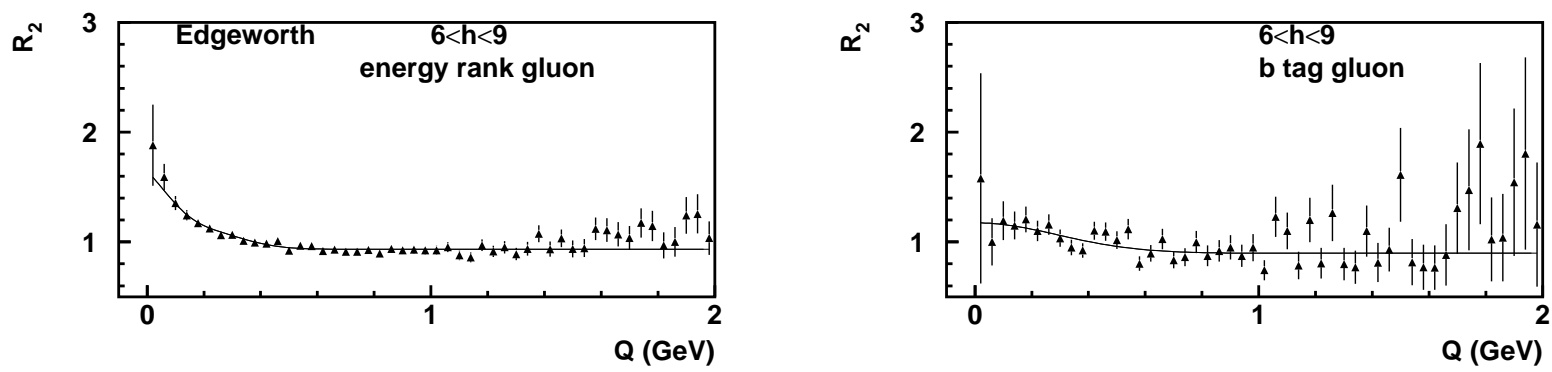

Figure 6.4: $R_{2}$ for gluon jets with hardness $6<h<9$ selected by energy rank (left) and by btagging (right). The line is the result of a fit of the Edgeworth parametrization. 

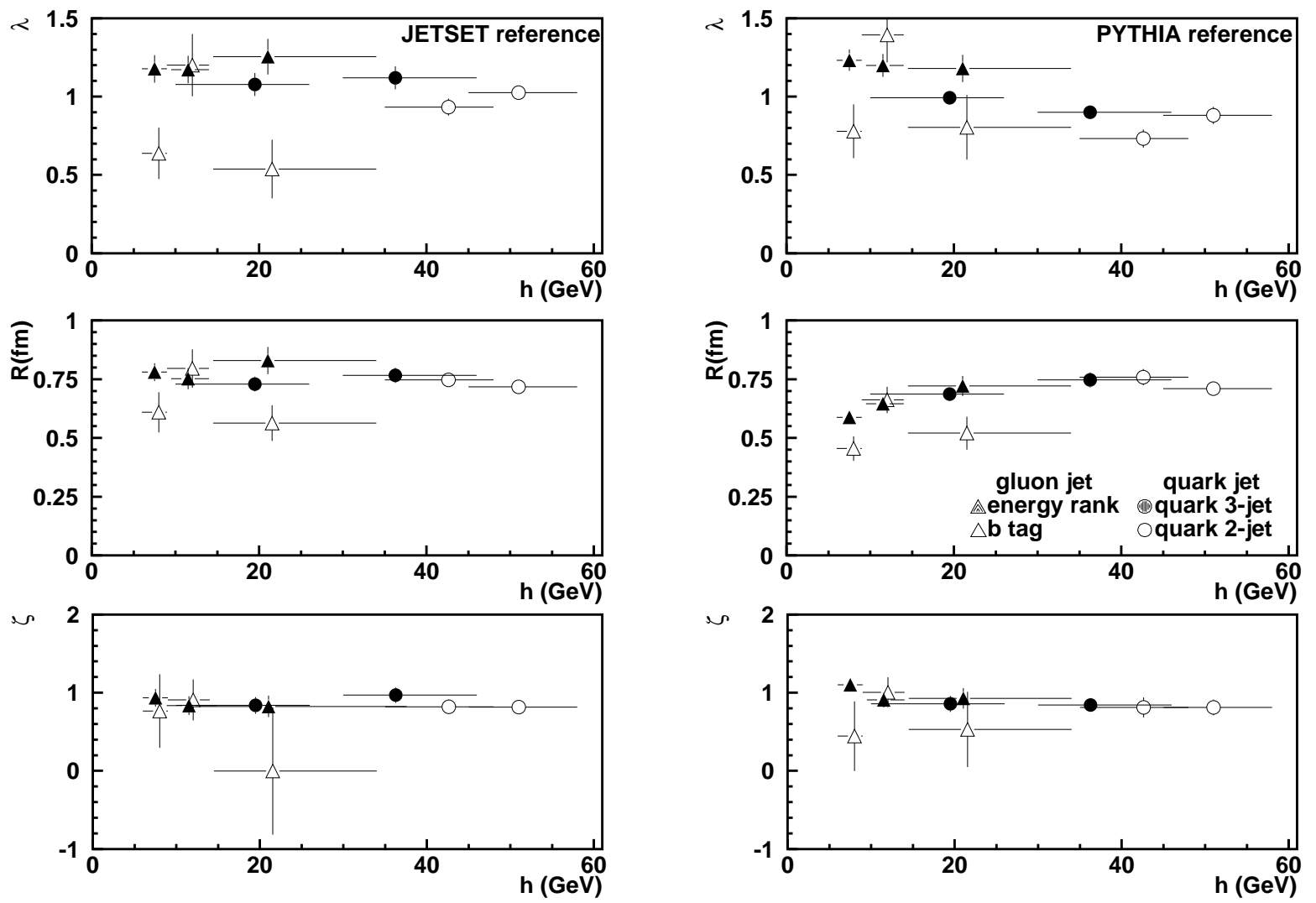

Figure 6.5: $\lambda, R$ and $\zeta$ for gluon and quark jets in different hardness ranges. Vertical error bars represent statistical errors and horizontal error bars indicate the range of hardness. JETSET (left three plots) and PYTHIA (right three plots) are used as the reference sample. Triangles are the results for gluon jets, where full triangles correspond to energy rank and open triangles to jet btagging. Solid circles are the results from quark jets in light-quark 3-jet events. Open circles are for the quark jets in light-quark 2 -jet events. $C_{\text {det }}$ is calculated using JETSET with BEC. 

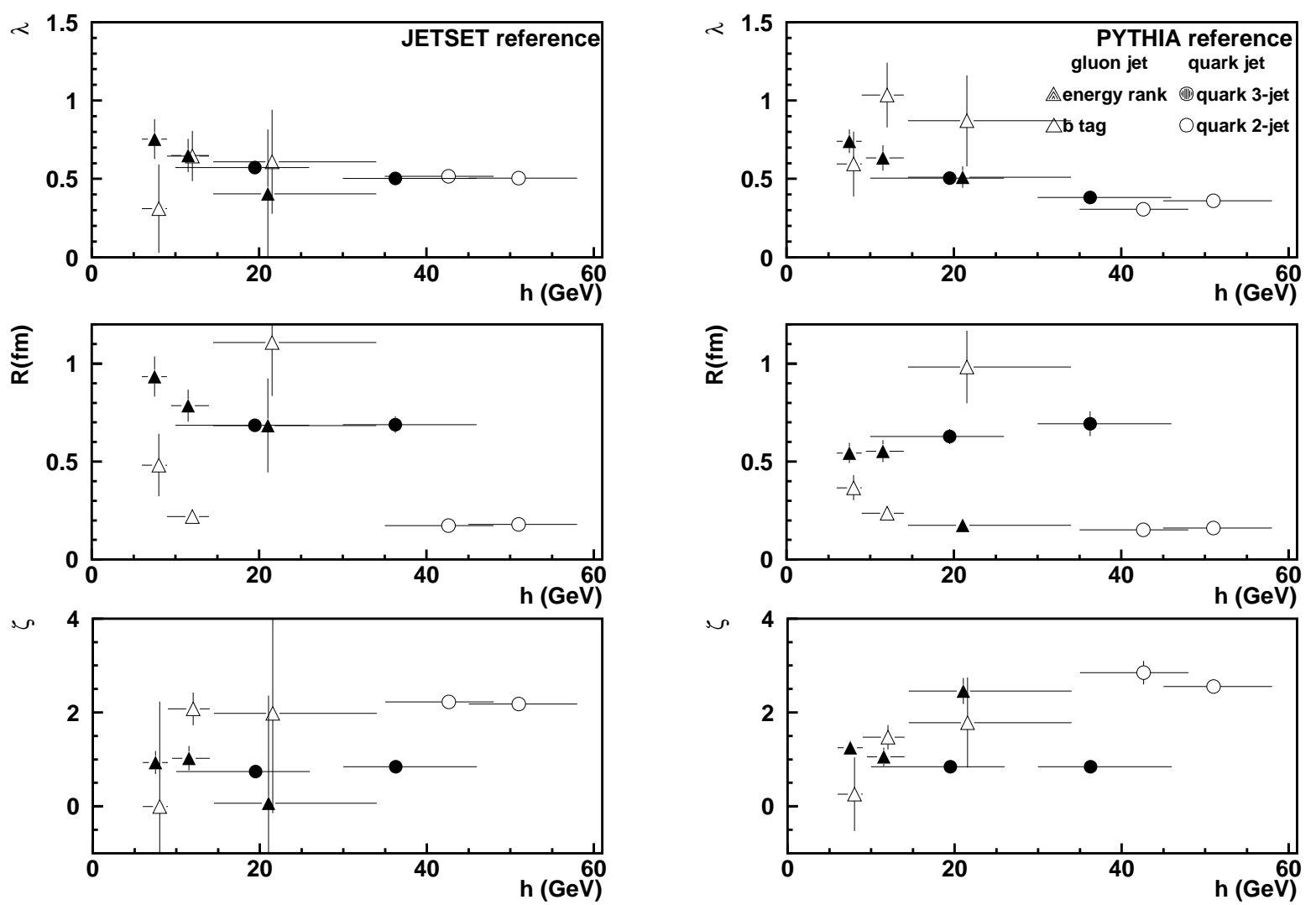

Figure 6.6: $\lambda, R$ and $\zeta$ for gluon and quark jets in different hardness ranges. Vertical error bars represent statistical errors and horizontal error bars indicate the range of hardness. JETSET (left three plots) and PYTHIA (right three plots) are used as the reference sample. Triangles are the results for gluon jets, where full triangles correspond to energy rank and open triangles to jet btagging. Solid circles are the results from quark jets in light-quark 3-jet events. Open circles are for the quark jets in light-quark 2-jet events. $C_{\text {det }}$ is calculated using JETSET without BEC.

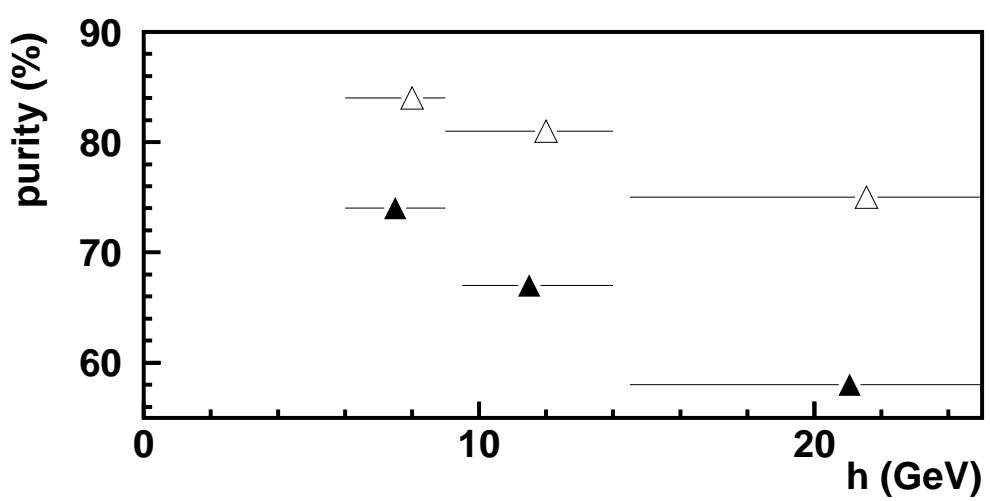

Figure 6.7: Purity of gluon jets in light-quark events (full triangles) and in b-quark events (open triangles) in different hardness ranges. The horizontal error bars indicate the range of hardness. 


\begin{tabular}{|c|c|c|c|c|}
\hline \multirow{2}{*}{ samples } & \multirow{3}{*}{ JETSET without BEC } \\
\cline { 3 - 5 } & average energy $(\mathrm{GeV})$ & $\lambda$ & $R(\mathrm{fm})$ & $\zeta$ \\
\hline 2QG sample (67\% quarks) & 29.3 & $1.25 \pm 0.11$ & $0.78 \pm 0.05$ & $1.06 \pm 0.13$ \\
\hline MG sample (74\% gluons) & 24.7 & $1.31 \pm 0.50$ & $0.96 \pm 0.21$ & $1.02 \pm 0.61$ \\
\hline \hline & & \multicolumn{3}{|c|}{ PYTHIA without BEC } \\
\cline { 3 - 5 } 2QG sample (67\% quarks) & 29.3 & $1.09 \pm 0.09$ & $0.73 \pm 0.04$ & $0.93 \pm 0.12$ \\
\hline MG sample (74\% gluons) & 24.7 & $1.38 \pm 0.44$ & $0.97 \pm 0.17$ & $1.12 \pm 0.53$ \\
\hline
\end{tabular}

Table 6.2: $\lambda, R$ and $\zeta$ for MG sample from b-quark events and 2QG sample from light-quark events. JETSET and PYTHIA without BEC are used as the reference samples.

\subsection{Comparison of Quark and Gluon Jets with Similar Energy}

In this section, $\lambda$ and $R$ are compared for quark and gluon jets of similar energy. Gluon jets are selected from specified topologies of 3-jet b-quark events: Mercedes events, Yq events and Yg events. Quark jets are from Mercedes events, Yq events and 2-jet events in the light-quark sample.

Parametrization of $R_{2}$ is done using the Edgeworth expansion Eq. (1.27) with parameters $\delta=\varepsilon=0$, since the low statistics of the Mercedes events are not enough to fit all 6 parameters. Here JETSET and PYTHIA without BEC are used as reference samples. $C_{\mathrm{det}}$ is calculated using JETSET with BEC. In most of the fits, the $\chi^{2}$ is reasonable. Of the 14 fits, three have $\chi^{2}$ greater than 56 (confidence level less than 10\%), in which case the errors of the parameters are scaled with $\sqrt{\chi^{2} / \mathrm{NDOF}}$. We continue using the Edgeworth expansion with parameters $\delta=\varepsilon=0$ to fit even though it gives a larger $\chi^{2}$ in some cases, since the comparison of $\lambda, R$ and $\zeta$ should be performed using the same fitting function.

\subsubsection{Quark and Gluon Jets in Mercedes Events}

The energy distributions of the gluon and the quark jets in both the light-quark and b-quark Mercedes events are shown in Fig. 6.8. Since the three jets in the light-quark Mercedes events have largely overlapping energy spectra, it is not reasonable to identify the quark jet using energy ranking in this type of event. All three jets of the light-quark Mercedes sample are used to obtain the $Q$ distribution. Thus, we compare this sample (2QG sample) of which $2 / 3$ of the jets are quarks to the gluon jet sample from b-quark Mercedes events (MG sample) having a gluon purity of $74 \%$. If there are differences in BEC between gluon and quark jets, there should be a difference between these two samples.

The extracted $\lambda, R$ and $\zeta$ values are listed in Table 6.2. We see that there is no significant difference between the two samples, no matter whether JETSET or PYTHIA without BEC is used as the reference sample. 

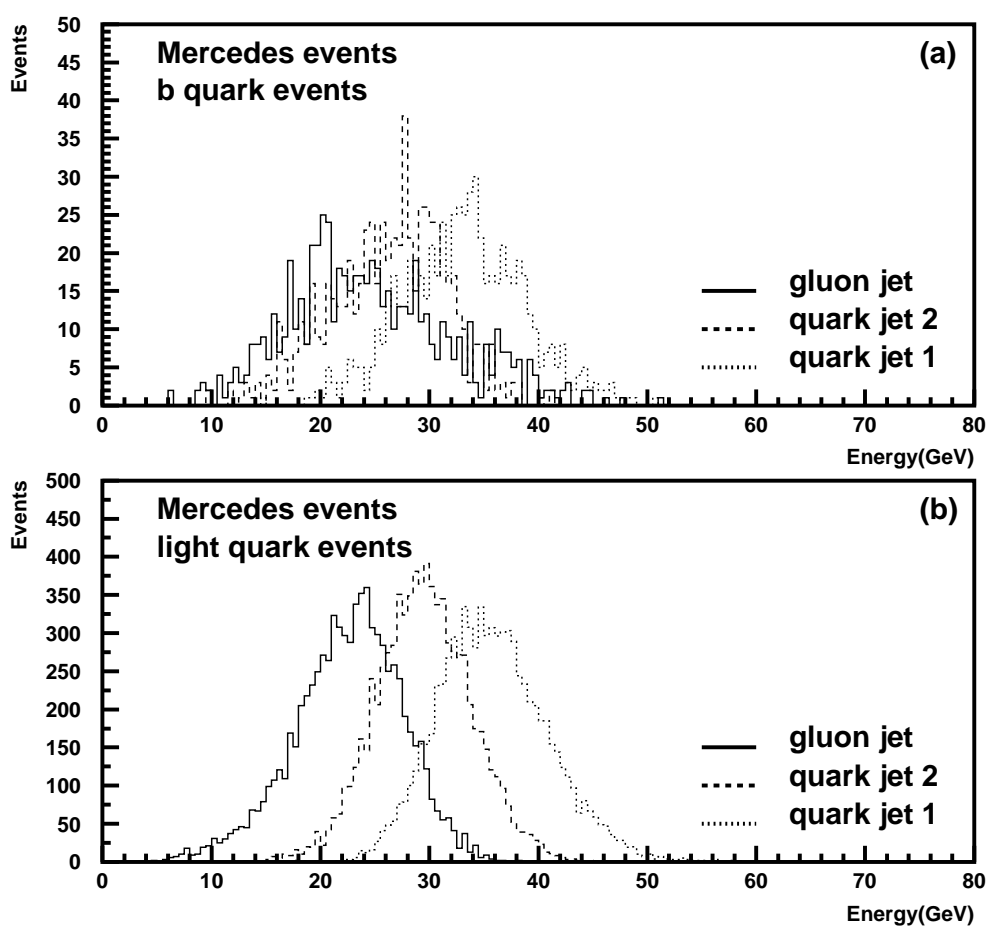

Figure 6.8: The energy distribution of the gluon and the quark jets in the Mercedes events of the data for the b-quark sample (a) and for the light-quark sample (b).

\subsubsection{Quark and Gluon Jets in Yq events}

The energy distributions of the gluon and the quark jets are shown in Fig. 6.9 for both the lightquark and b-quark Yq events. The energy distributions of the two least energetic jets in the lightquark Yq events have a large overlap. So the comparison of quark and gluon jets in the Yq events is performed by comparing the gluon jet from the b-quark Yq events (YqG sample) to the two least energetic (1 quark +1 gluon) jets in the light-quark Yq events (1QG sample).

The extracted $\lambda, R$ and $\zeta$ values are listed in Table 6.3. JETSET and PYTHIA without BEC are used as reference samples. $C_{\text {det }}$ is calculated using JETSET with BEC. Table 6.3 shows that $\lambda$ and $R$ for the two samples are both consistent within statistical errors, no matter whether JETSET or PYTHIA without BEC are used as the reference sample.

\subsubsection{Gluon Jet in the Yg Events and Quark Jet in the 2-jet Events}

In this section, the gluon jet is selected from the Yg events, and quark jets are from the light-quark 2-jet events.

The selection of gluon jets in Yg events is described in Sect. 3.7 and Sect. 3.8.3. After the selection, there are 939 gluon jets from Yg events with purity $32 \%$.

The energy distribution of the gluon and the quark jets in the b-quark Yg events is shown in Fig. 6.10. As can be seen there, enough gluon jets exist with high energy.

Fig. 6.11 compares the energy distribution of gluon jets in the Yg events and the quark jets in 

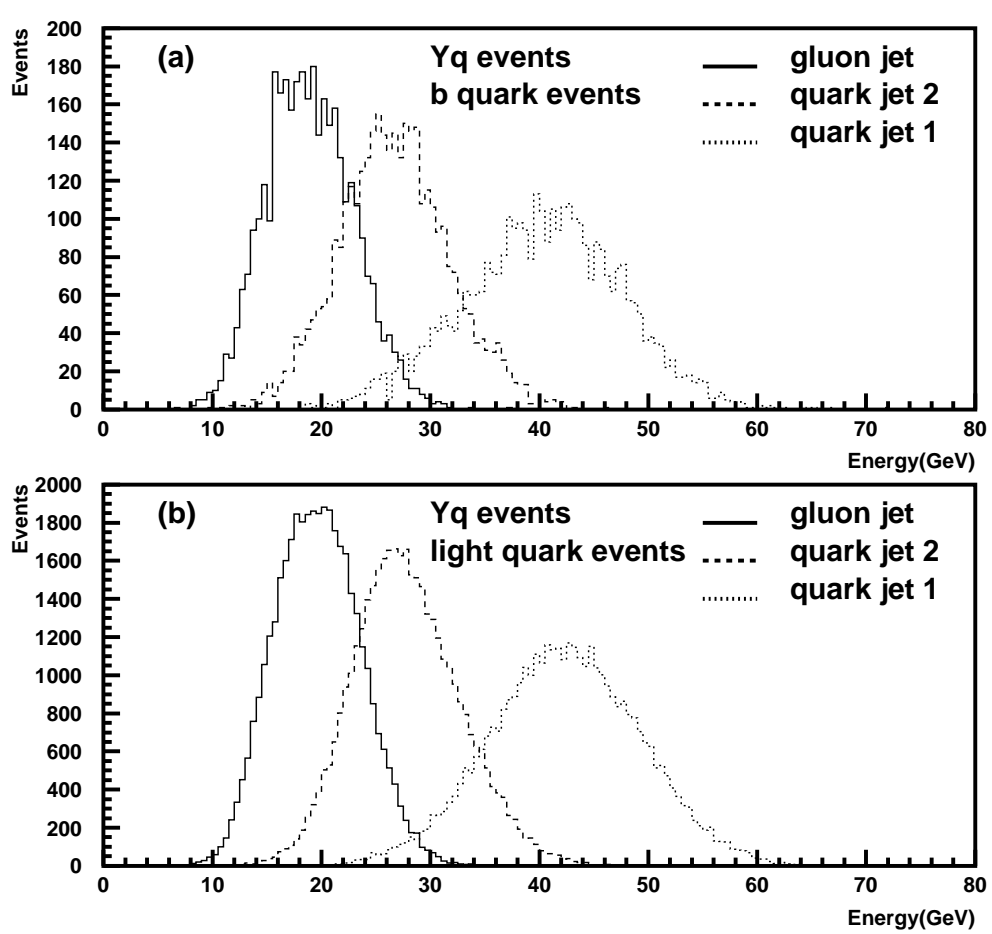

Figure 6.9: The energy distribution of the gluon and the quark jets in the Yq events of the data for the b-quark sample (a) and for the light-quark sample (b).

\begin{tabular}{|c|c|c|c|c|}
\hline \multirow{2}{*}{ sample } & \multirow{3}{|c|}{ JETSET without BEC } \\
\cline { 3 - 5 } & average energy $(\mathrm{GeV})$ & $\lambda$ & $R(\mathrm{fm})$ & $\zeta$ \\
\hline 1QG sample (50\% quarks) & 23.7 & $1.13 \pm 0.09$ & $0.78 \pm 0.04$ & $0.83 \pm 0.12$ \\
\hline YqG sample (75\% gluon) & 18.9 & $0.83 \pm 0.18$ & $0.76 \pm 0.09$ & $0.55 \pm 0.37$ \\
\hline \hline & & \multicolumn{3}{|c|}{ PYTHIA without BEC } \\
\cline { 3 - 5 } 1QG sample (50\% quarks) & 23.7 & $1.09 \pm 0.06$ & $0.68 \pm 0.03$ & $0.91 \pm 0.09$ \\
\hline YqG sample (75\% gluon) & 18.9 & $1.07 \pm 0.17$ & $0.65 \pm 0.06$ & $0.85 \pm 0.26$ \\
\hline
\end{tabular}

Table 6.3: $\lambda, R$ and $\zeta$ for the YqG sample from b-quark Yq events and the 1QG sample from light-quark Yq events. JETSET and PYTHIA without BEC are used as the reference samples. 


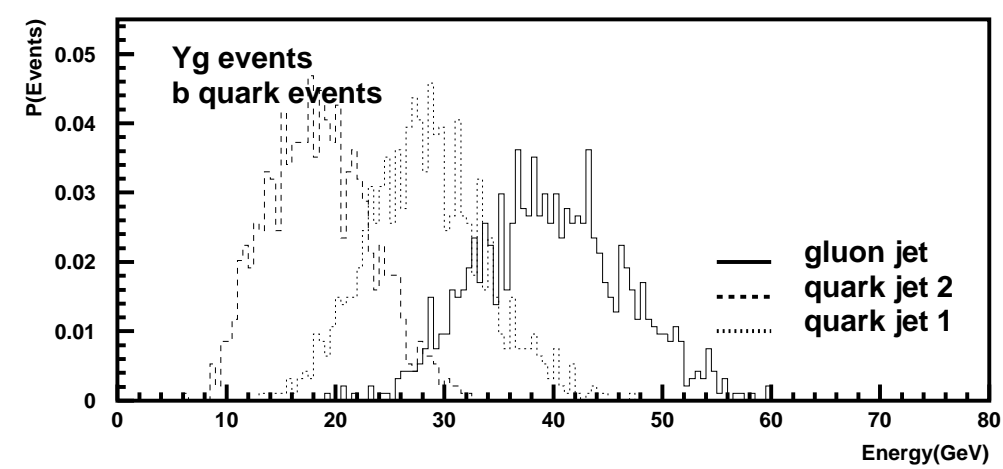

Figure 6.10: The energy distribution of the gluon and the quark jets in the b-quark Yg events of the data.

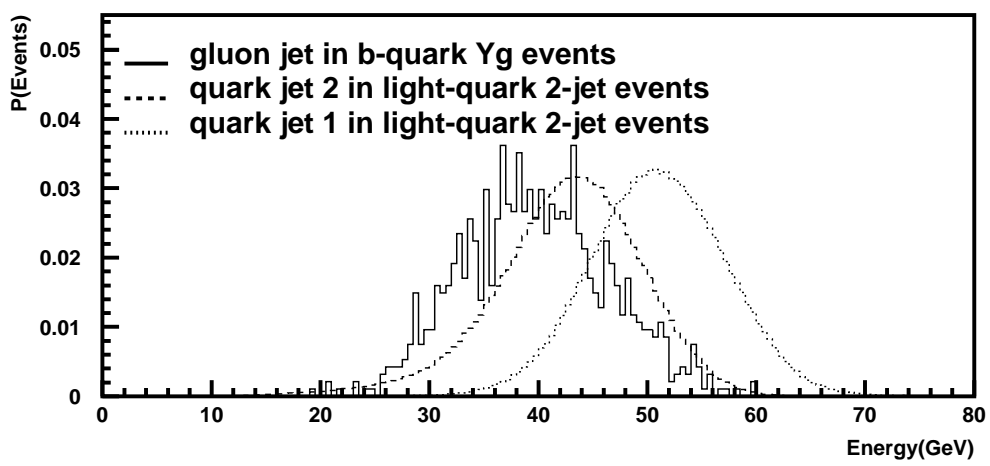

Figure 6.11: The energy distribution of the gluon jets in the b-quark Yg events and the quark jets of the data in the light-quark 2 -jet events.

the light-quark 2-jet events. There is more overlap in the energy distribution of the gluon with that of the second most energetic quark jet than with that of the most energetic quark. Therefore only the second most energetic quark jet is used to compare $\lambda$ and $R$ in Table 6.4. Again, JETSET and PYTHIA without BEC are used as reference samples. $C_{\text {det }}$ is calculated using JETSET with BEC.

From Table 6.4, we can see that $R$ is smaller in the gluon jet than in the quark jet when JETSET without BEC is used as reference sample, but it shows no difference when using PYTHIA without BEC as reference sample. $\lambda$ is smaller in the gluon jet than in the quark jet when using JETSET without BEC as reference sample, while $\lambda$ is larger in the gluon jet when using PYTHIA without $\mathrm{BEC}$ as reference sample. Taken all together, no differences can be claimed to be present.

\subsection{Rapidity and $x$ Dependence of $\lambda$ and $R$ in Quark and Gluon Jets}

Since we do not see any significant dependence on hardness or energy, we continue the comparison of quark and gluon jets ignoring the energy difference. We now study $\lambda$ and $R$ of quark and gluon jets using only particles in a particular window of $x$ and rapidity $y$, where $x$ and $y$ are defined as 


\begin{tabular}{|c|c|c|c|c|}
\hline \multirow{2}{*}{ sample } & \multicolumn{4}{|c|}{ JETSET without BEC } \\
\cline { 3 - 5 } & average energy $(\mathrm{GeV})$ & $\lambda$ & $R(\mathrm{fm})$ & $\zeta$ \\
\hline quark jet (100\% quark) & 42.6 & $0.93 \pm 0.05$ & $0.75 \pm 0.03$ & $0.82 \pm 0.09$ \\
\hline gluon jet (32\% gluon) & 39.7 & $0.96 \pm 0.24$ & $0.56 \pm 0.10$ & $0.65 \pm 0.43$ \\
\hline \hline \multirow{3}{*}{ quark jet (100\% quark) } & 42.6 & \multicolumn{3}{|c|}{ PYTHIA without BEC } \\
\cline { 3 - 5 } gluon jet (32\% gluon) & 39.7 & $0.73 \pm 0.06$ & $0.76 \pm 0.04$ & $0.81 \pm 0.12$ \\
\hline
\end{tabular}

Table 6.4: $\lambda, R$ and $\zeta$ for the gluon jets in the b-quark Yg events and the second most energetic quark jets in the light-quark 2-jet events. JETSET and PYTHIA without BEC are used as the reference samples.

$$
\begin{gathered}
x=p_{z} / E_{\mathrm{jet}}, \\
y=\frac{1}{2} \ln \frac{E+p_{z}}{E-p_{z}},
\end{gathered}
$$

with $p_{z}$ the component of the particle's momentum along the jet axis, $E_{\text {jet }}$ the energy of the jet and $E$ the energy of the particle.

The distributions of $x$ and $y$ are shown in Fig. 6.12. Three regions of $x$ and $y$ are chosen to study the $x$ and $y$ dependence: $x \geq 0.0375,0.018 \leq x \leq 0.075$ and $x \leq 0.0375 ; y \geq 1.5,0.8 \leq y \leq 2.0$ and $y \leq 1.5$.

Since there is more overlap between the strings in the tip of the gluon jet than in the central region, we expect the quark-gluon differences to increase with increasing $x$ and $y$.

Fig. 6.13 shows the values of $\lambda, R$ and $\zeta$ as a function of $x$ for quark and gluon jets. The quark jets are from light-quark 3 -jet events. The gluon jet is identified using energy rank or b-tagging, the latter having a somewhat smaller value of $\lambda$. The two quark jets, which cover different hardness ranges, show consistent results. For both quark and gluon jets, $\lambda$ shows some $x$ dependence, while $R$ does not. Considering the dependence on the gluon jet identification method, neither $\lambda$ nor $R$ shows significant difference between quark and gluon jets.

Fig. 6.14 shows the values of $\lambda, R$ and $\zeta$ as a function of $y$ for quark and gluon jets. $\lambda$ shows some $y$ dependence, while $R$ does not. However, also as a function of $y$, the values of $\lambda$ and $R$ show no significant difference between quark and gluon jets.

\subsection{Conclusion}

In this chapter, firstly the hardness dependence has been studied while comparing quark and gluon jets. No obvious hardness dependence has been observed.

Nevertheless, we, secondly, have restricted the comparison to quark and gluon jets of similar energy. Similar-energy quark and gluon jets show no significant differences in correlation strength $\lambda$ or radius parameter $R$ for Mercedes events, Yq and Yg events. 

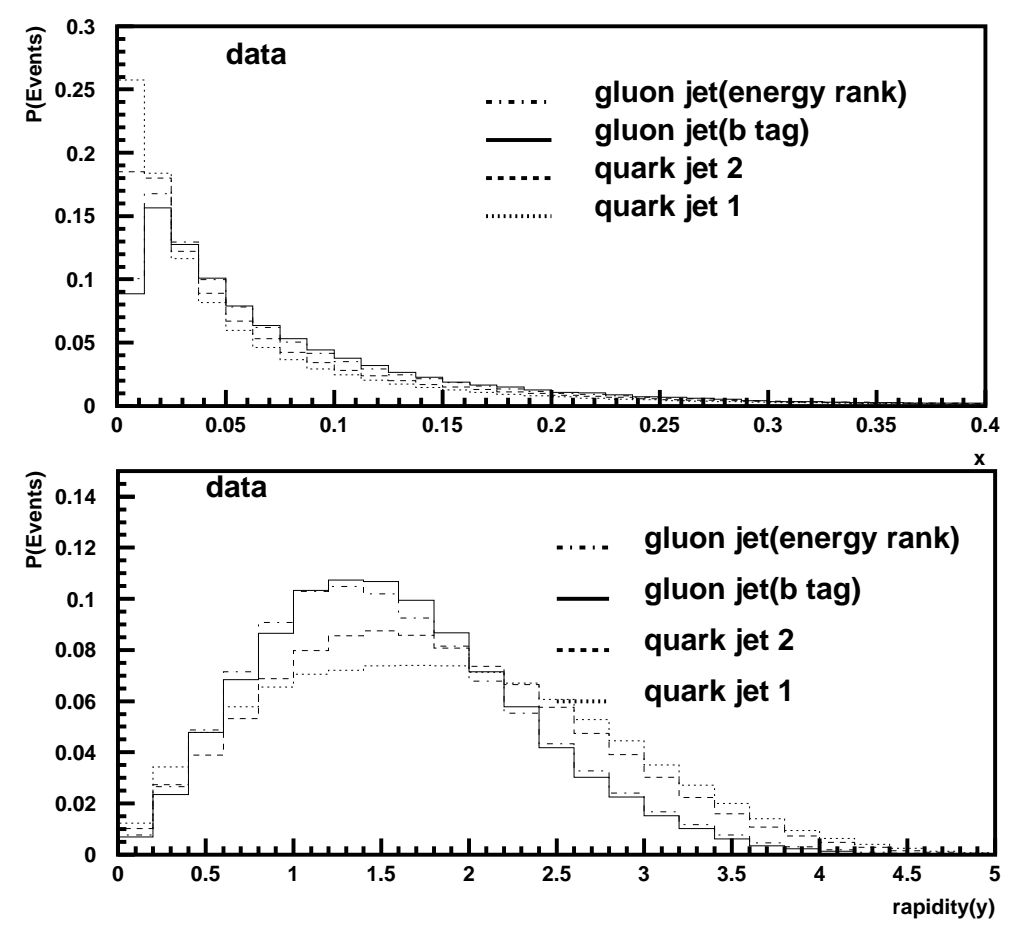

Figure 6.12: $x$ (above) and $y$ (below) distribution for gluon jets (dash-dotted line using energy rank and solid line using double b-tagging), second-most energetic quark jets (dashed line) and most energetic quark jets (dotted line) in the data sample.

Thirdly, now ignoring the energy difference of jets, $\lambda$ and $R$ have been studied as a function of rapidity $y$ and of the variable $x$ of particles within the jets. While $R$ does not depend on these variables, $\lambda$ shows some $x$ and $y$ dependence, but neither $\lambda$ nor $R$ shows any significant difference between quark and gluon jets.

We conclude that no significant difference between quark and gluon jets is observed in our Bose-Einstein Correlation study. 

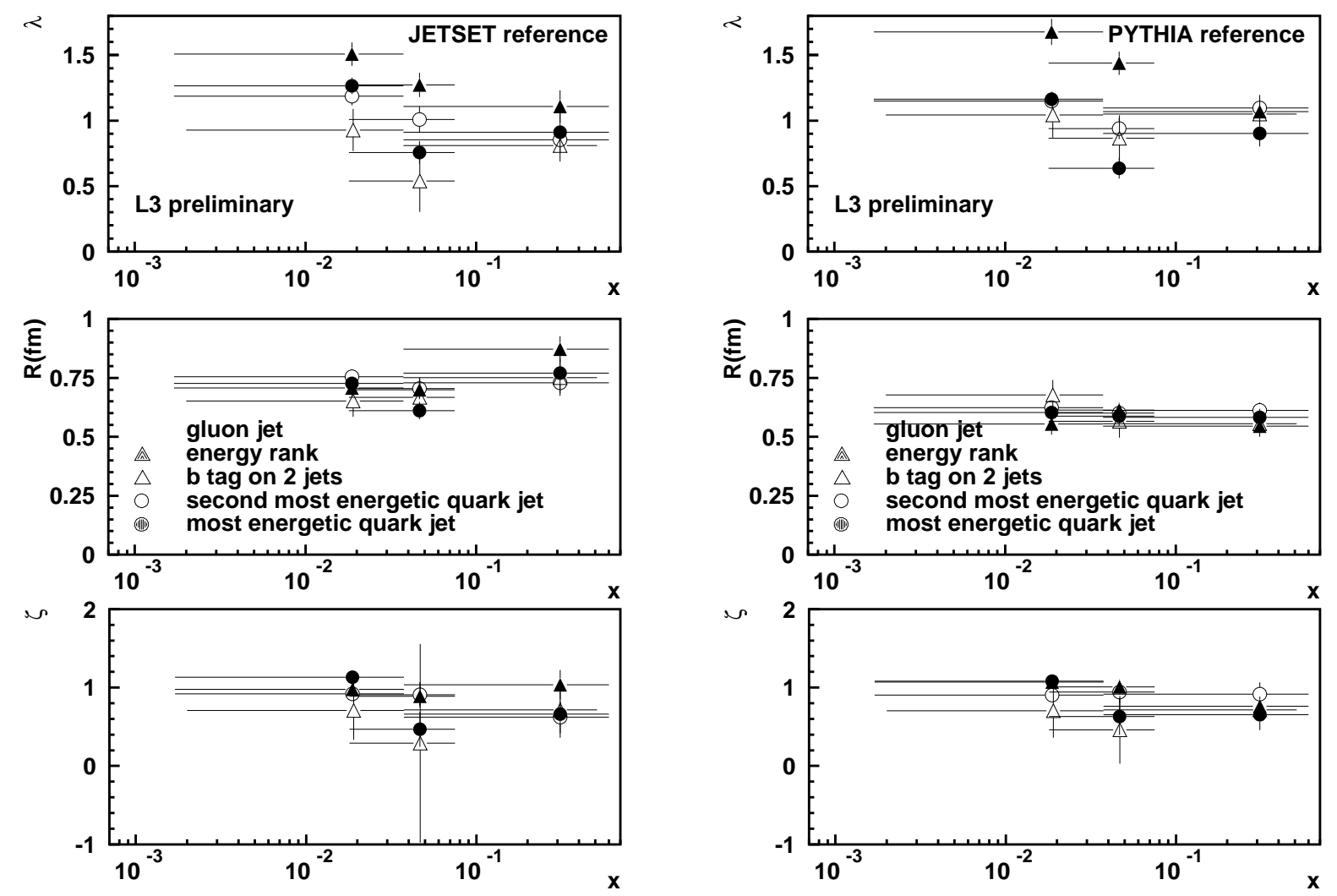

Figure 6.13: $\lambda, R$ and $\zeta$ vs. $x$ for the gluon jet (triangles) and quark jet (circles) using JETSET (left two plots) and PYTHIA (right two plots) as the reference sample. Vertical error bars represent statistical errors and horizontal error bars indicate the range of $x$. The quark jets are the second most energetic quark jet (open circles) and most energetic quark jet (solid circles) in the lightquark 3 -jet events. The gluon jet is identified by energy rank (solid triangles) and b-tagging (open triangles). 

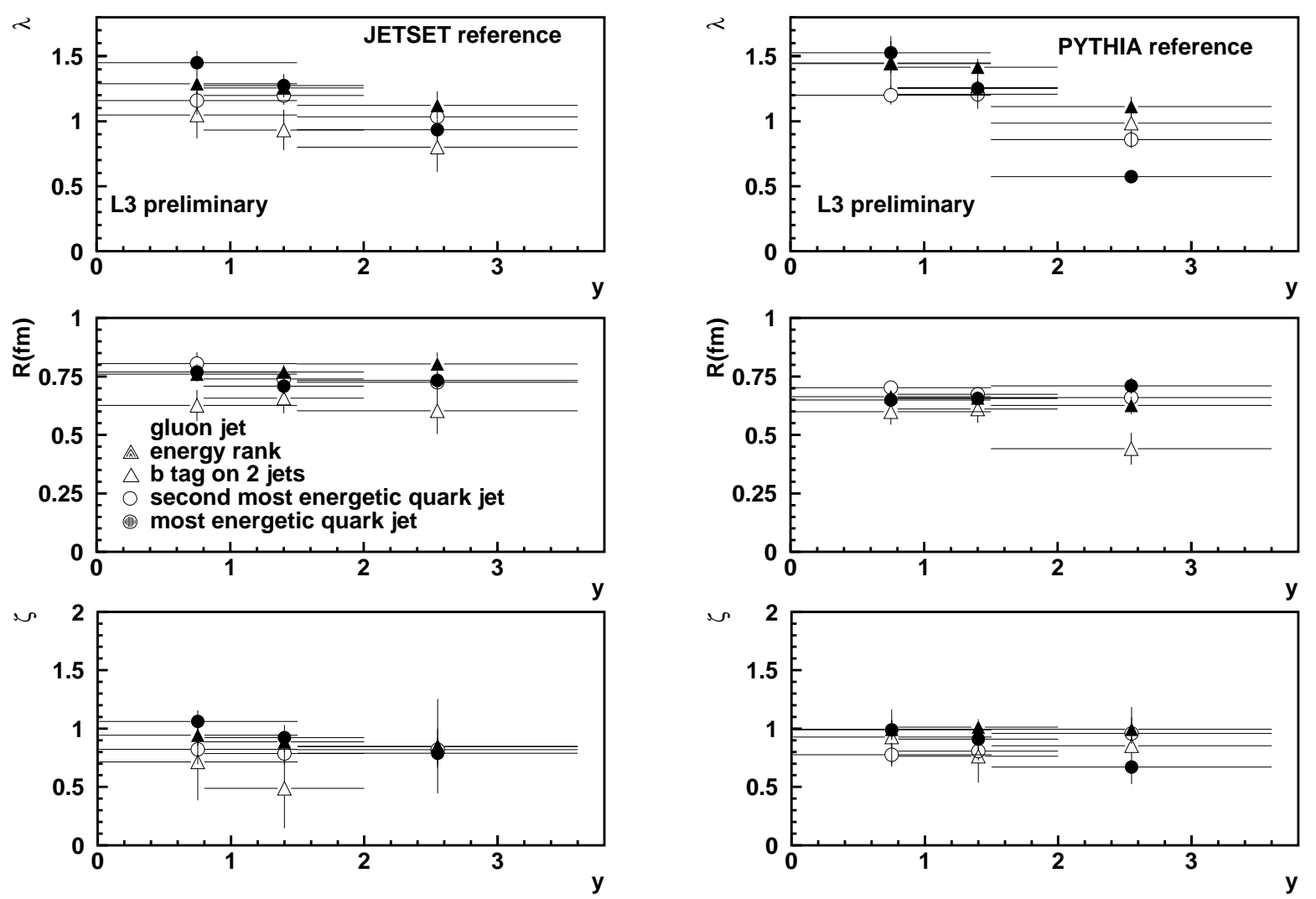

Figure 6.14: $\lambda, R$ and $\zeta$ vs. $y$ for the gluon jet (triangles) and quark jet (circles) using JETSET (left two plots) and PYTHIA (right two plots) as the reference sample. Vertical error bars represent statistical errors and horizontal error bars indicate the range of $y$. The quark jets are the second most energetic quark jet (open circles) and most energetic quark jet (solid circles) in the lightquark 3-jet events. The gluon jet is identified by energy rank (solid triangles) and b-tagging (open triangles). 


\section{Chapter 7}

\section{Comparison of Same Side and Different Sides of Gluon Jet}

In this chapter, the correlation function $R_{2}$ is measured using pairs of particles from the same side of a gluon jet and particles from different sides of a gluon jet. Particles from the same side of the gluon jet are expected to originate from the same string, while particles from different sides are expected to originate from different strings. Inter-string BEC is thus studied in a more direct way by comparing pairs of particles originating from the same side with those where one particle originates from each side of the gluon jet.

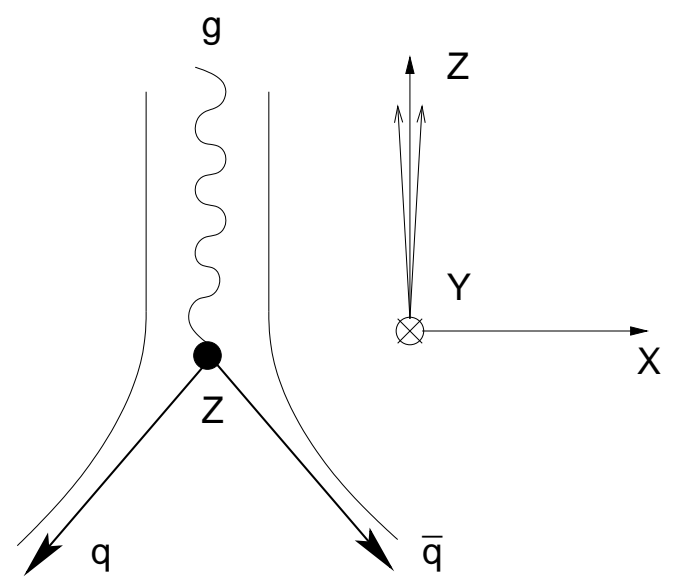

Figure 7.1: Illustration of the new frame in the event plane of 3-jet events.

To decide whether particles originate from the same or from different sides of a gluon jet, particles are required to be in the event plane, i.e., the plane in which the 3 jets are emitted. A new frame $(X, Y, Z)$ is defined as shown in Fig. 7.1. The $Z$ axis is in the event plane and along the gluon jet direction. The $Y$ axis points out of the event plane and the $X$ axis is the cross product of the $Y$ and $Z$ axes. The four-momenta of jets and particles are transformed into this new frame. Particles are judged whether they originate from the same or from different sides of the gluon by the sign of their $X$ component of momentum. Particles with the same sign are considered to originate from 
the same side, i.e., from the same string, whereas particles with different sign from different sides, i.e., from different strings.

Because of the limited average multiplicity at LEP, the multiplicity in the gluon jets is quite small and the number of pairs from the same and from different sides of gluon jets even smaller. The low statistics can cause difficulty in observing the possible BEC signal. Thus, firstly, the feasibility of this study is checked. This is done at MC generator level. Secondly, the correlation strength $\lambda$ from particle pairs from the same side and from different sides of the gluon jet are compared, in all 3-jet events and in three special types of 3-jet event: the Yq events, Mercedes events and Yg events. Finally, rapidity and $x$ (as defined in Eq. (6.2)) dependence are studied.

\subsection{Feasibility of Studying Same Side and Different Sides of a Gluon Jet}

We first perform a feasibility study at Monte Carlo generator level. The Durham jet algorithm with $y_{\text {cut }}=0.02$ is applied using all particles to select 3 -jet events and to determine jet directions and energies. Which jet is the gluon jet is decided by energy rank for light-quark events and by double b-tagging for heavy-quark events. The charged particles are then reassigned to the jets using the angles between the particle and the jets, the particle being assigned to the closest jet, as is done for data (see Sect. 3.6). To guarantee that the particles assigned to the gluon jet really originate from the gluon, the gluon must be sufficiently separated from the quarks. This separation is studied in Sect. 7.1.1.

Because of the jet algorithm, the reconstructed gluon jet direction and the original gluon direction are not identical. This difference causes some particles to be assigned to the wrong side of the gluon jet. This is studied in Sect. 7.1.2.

The feasibility of using $R_{2}$ to compare the same side and different sides of the gluon jet is studied by using pairs of particles originating from the same side and pairs of particles originating from different sides of the gluon jet in Sect. 7.1.3.

\subsubsection{Check of the Separation of Gluon and Quark Jets at Generator Level}

The distribution of the angle in the event plane between the gluon direction and the projected momentum of the particles originating from the two strings (solid and dashed lines) for $\mathrm{q} \overline{\mathrm{q}} \mathrm{g}$ events is shown in Figs. 7.2(a)-(f). The matrix element Monte Carlo JETSET ME is used to generate the particles. The two strings are between the gluon and the higher-energy (string 1) and lower-energy (string 2) quarks. The original gluon direction is taken as the $0^{\circ}$ axis.

Fig. 7.2(a) is for the entire gluon energy range $0-45.6 \mathrm{GeV}$, and Figs. 7.2(b)-(f) are for different gluon energy ranges: $0-4,4-8.5,8.5-15,15-25$ and $25-45.6 \mathrm{GeV}$, respectively. The figures show how well the gluon jet is separated from the quark jets. The seperation is seen to improve as the energy of the gluon jet increases. The width of the gluon jet is $\pm 10^{\circ}$ at half height. The figures also show that particles originating from different strings can appear on the same side of the gluon jet. The fraction of particles on the same side of the gluon as the string from which they originate 
is seen to vary with the energy of the gluon and the angle between the particles and the gluon.
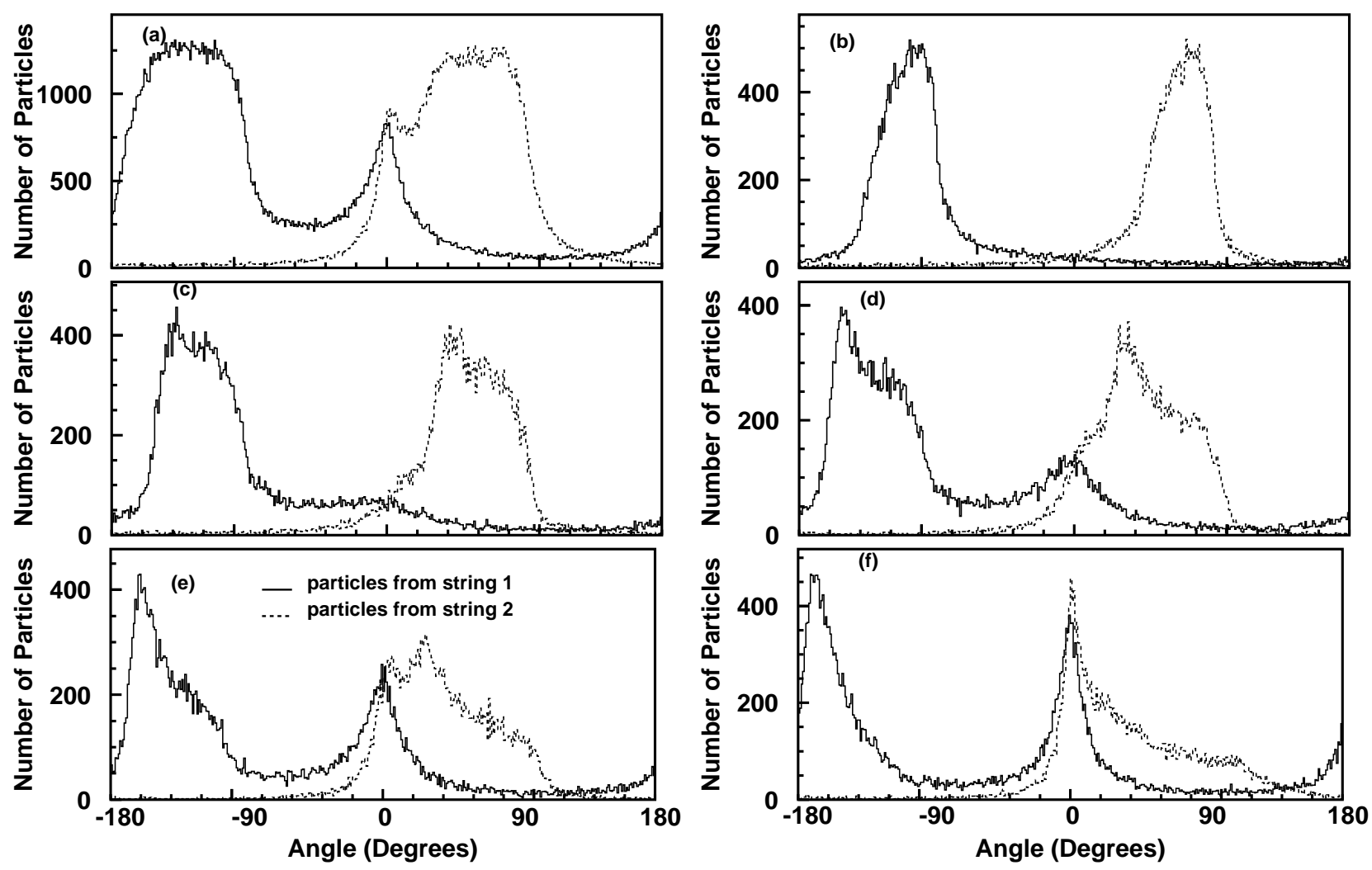

Figure 7.2: Particle flow with respect to the angle in the event plane between the projection of the particle's momentum and the gluon direction for q $\bar{q} g$ events generated by JETSET ME. The solid (dashed) line is for particles originating from the string between the gluon and the higher (lower) energy quark. The gluon has energy $0-45.6 \mathrm{GeV}$ in (a), $0-4 \mathrm{GeV}$ in (b), 4-8.5 GeV in (c), $8.5-15 \mathrm{GeV}$ in (d), $15-25 \mathrm{GeV}$ in (e), $25-45.6 \mathrm{GeV}$ in (f).

\subsubsection{Check of the Deviation of the Jet Direction from the Gluon Direction}

In order to estimate the deviation of the reconstructed jet direction from the original gluon direction, JETSET ME without BEC is used to generate a sample at generator level. The Durham algorithm is used to reconstruct the jets using all (also neutral) particles. The deviation between the jet direction and the gluon direction is calculated by the following method:

There are 6 combinations of the 3 partons and 3 jets. The sum of the angles between the partons and the jets is calculated for each of the 6 combinations. The best match is taken as the combination with the smallest sum. The angle between the gluon direction and the closest jet in this combination, $\delta \sigma_{\text {gluon-jet }}$, is plotted in Fig. 7.3 , for all gluons as well as for gluons in various energy ranges. The average deviation is around $3.4^{\circ}$ for all gluons, decreasing from $4.8^{\circ}$, to $3.0^{\circ}$ to $2.0^{\circ}$ with gluon energy ranges increasing from $8.5-15 \mathrm{GeV}, 15-25 \mathrm{GeV}$ to $25-45.6 \mathrm{GeV}$. This means that those particles with an angle to the gluon jet less than $4.8^{\circ}, 3.0^{\circ}$ or $2.0^{\circ}$, respectively, 
have ambiguity as to the side of the jet from which they originate. This will be taken into account in the analysis. In data the deviation will be somewhat larger due to the experimental jet angular resolution of about $2.5^{\circ}$ [76].
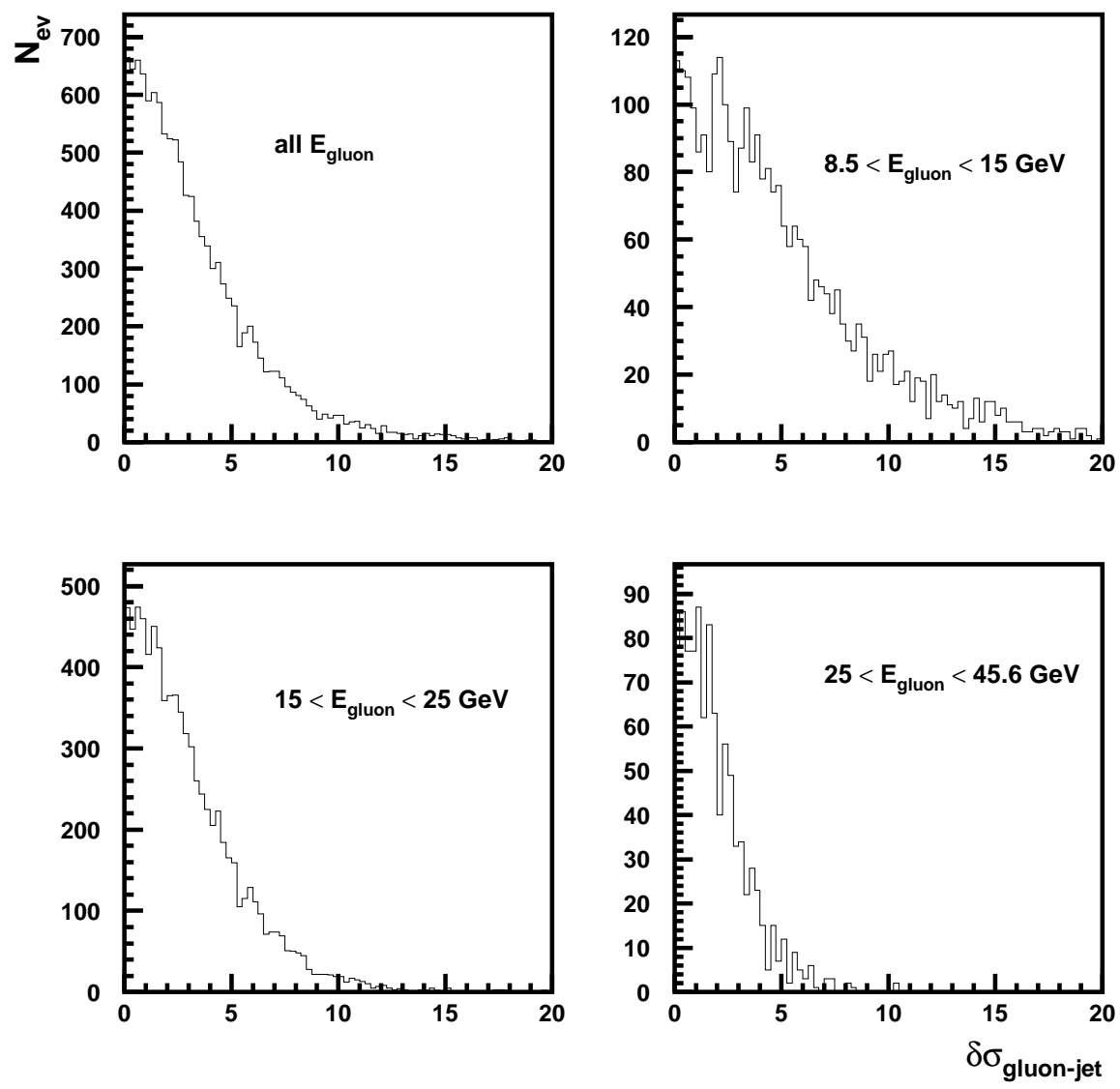

Figure 7.3: The absolute value of the deviation of the jet direction from the gluon direction $\delta \sigma_{\text {gluon-jet }}$, for all gluons (upper left), and for gluons in the energy range $8.5-15 \mathrm{GeV}$ (upper right), $15-25 \mathrm{GeV}$ (lower left) and 25-45.6 GeV (lower right).

\subsubsection{Feasibility of Using $\boldsymbol{R}_{2}$ to Compare the Same Side and Different Sides of the Gluon Jet}

PYTHIA at generator level is used to check the feasibility of seeing differences in BEC by comparing $R_{2}$ for pairs of pions from the same side of the gluon to $R_{2}$ for pairs of pions from different sides. Fig. 7.4 shows the $Q$ distribution using particle pairs originating from the same side and from different sides of the gluon jet. Both PYTHIA with and without BEC are shown. $R_{2}$ is calculated using PYTHIA with BEC divided by PYTHIA without BEC. Indeed BEC show up in the low- $Q$ area both for the same side and for different sides of the gluon jet. However, at low- $Q$ the number of particle pairs for different sides of the gluon is much lower than that for the same side, which is reflected in the much larger statistical uncertainty of $R_{2}$. This is the case for both PYTHIA with 
and without BEC. In principle, no difference in PYTHIA without BEC might be expected between the pair density distibution of same-side pairs and different-side pairs. In practice, however, the angle between tracks from different sides is larger than that from the same side. An extreme situation is that $0^{\circ}$ is impossible for different sides. This means that pairs from the same side dominate in the low- $Q$ region, while pairs from different sides dominate in the high- $Q$ area.
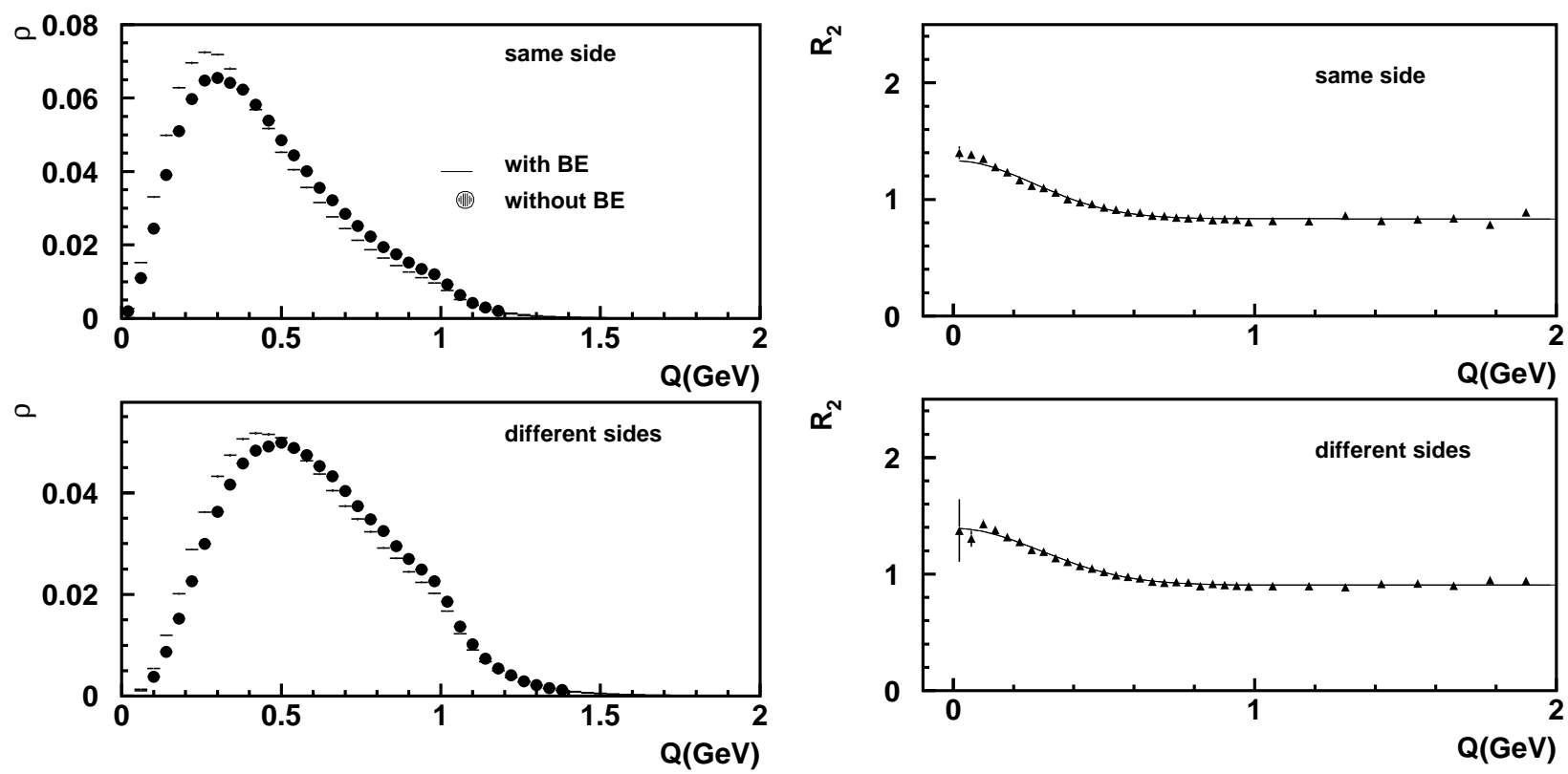

Figure 7.4: Normalized $Q$ distribution (left two plots) using particle pairs from the same side (above) and different sides (below) of the gluon jet. PYTHIA with BEC (solid lines) and without BEC (solid circles) are shown. $R_{2}=\rho_{\text {with BE} /} \rho_{\text {without BE }}$ is shown in the right two plots, with the results of a fit of Eq. (1.27) with parameters $\delta=\varepsilon=\zeta=0$. The fitted line is shown to guide the eye.

Figs. 7.5-7.7 and Figs. 7.8-7.10 show the $Q$ and $R_{2}$ distributions in different $x$ and $y$ regions, respectively. BEC of same-side and different-side particle pairs can be seen under all these conditions. We also see that the number of different-side particle pairs decreases with increasing $x$ and $y$.

From the MC study of Sect. 7.1, we conclude that it is possible to use $R_{2}$ to compare same-side and different-side particle pairs.

\subsection{Comparison of the Same Side and Different Sides of the Gluon Jet}

We turn now to the experimental data. Because of the limited efficiency of detecting particles and the resolution of the calorimeters, there is energy imbalance and the 3 jets found by the jet algorithm are not in the event plane. Therefore, the 3 jets found by the Durham jet algorithm with $y_{\text {cut }}=0.02$ are forced into the same plane using the momentum imbalance. One third of this imbalance is subtracted from each jet. Then the new direction of the gluon jet is taken as the new 

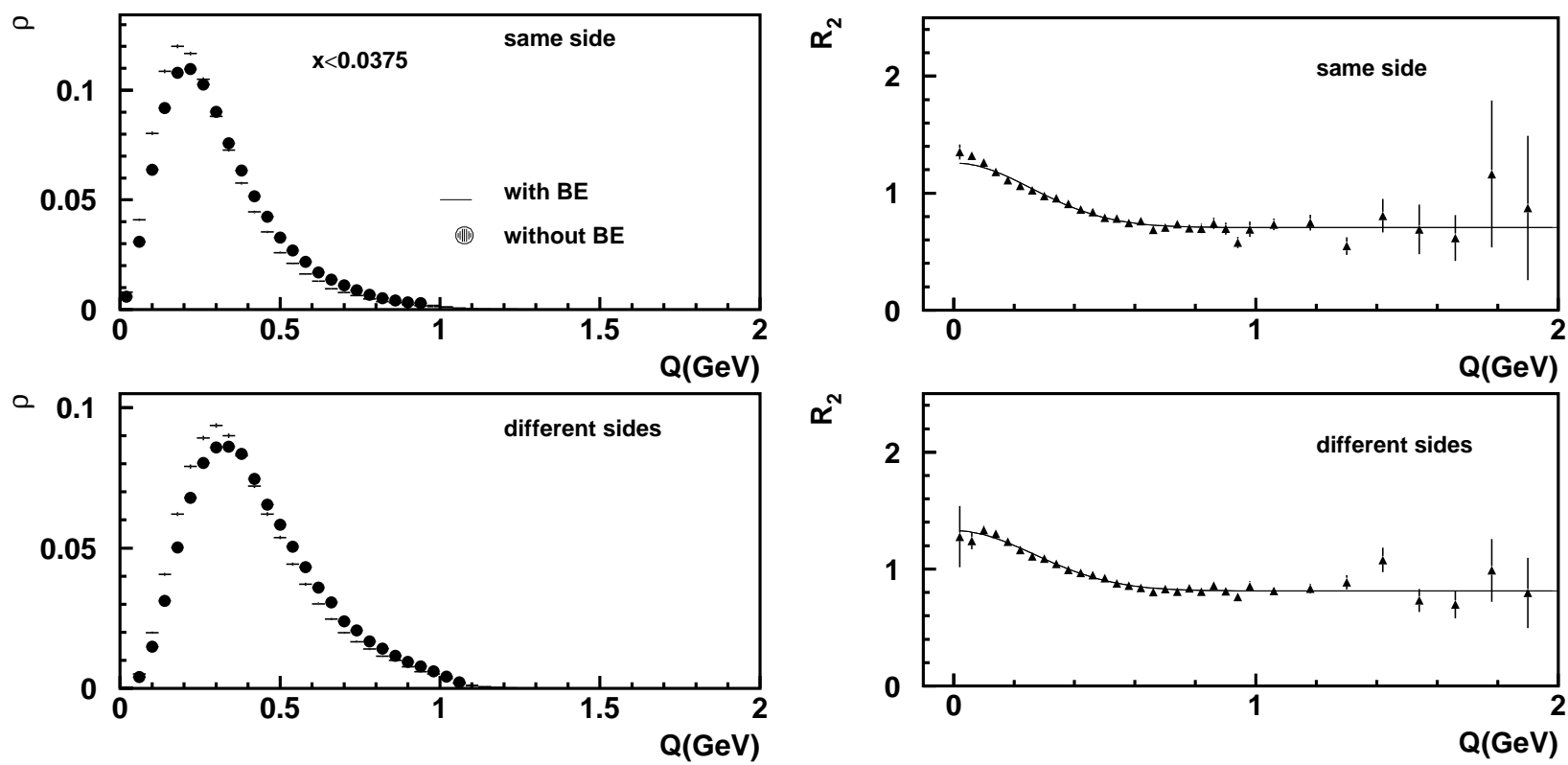

Figure 7.5: Normalized $Q$ distribution (left two plots) using particle pairs from the same side (above) and different sides (below) of the gluon jet. PYTHIA with BEC (solid lines) and without $\mathrm{BEC}$ (solid circles) are shown. $R_{2}=\rho_{\text {with BE}} / \rho_{\text {without BE }}$ is shown in the right two plots, with the results of a fit of Eq. (1.27) with parameters $\delta=\varepsilon=\zeta=0$. Only particles with $x$ in the region of $x<0.0375$ are used. The fitted line is shown to guide the eye.
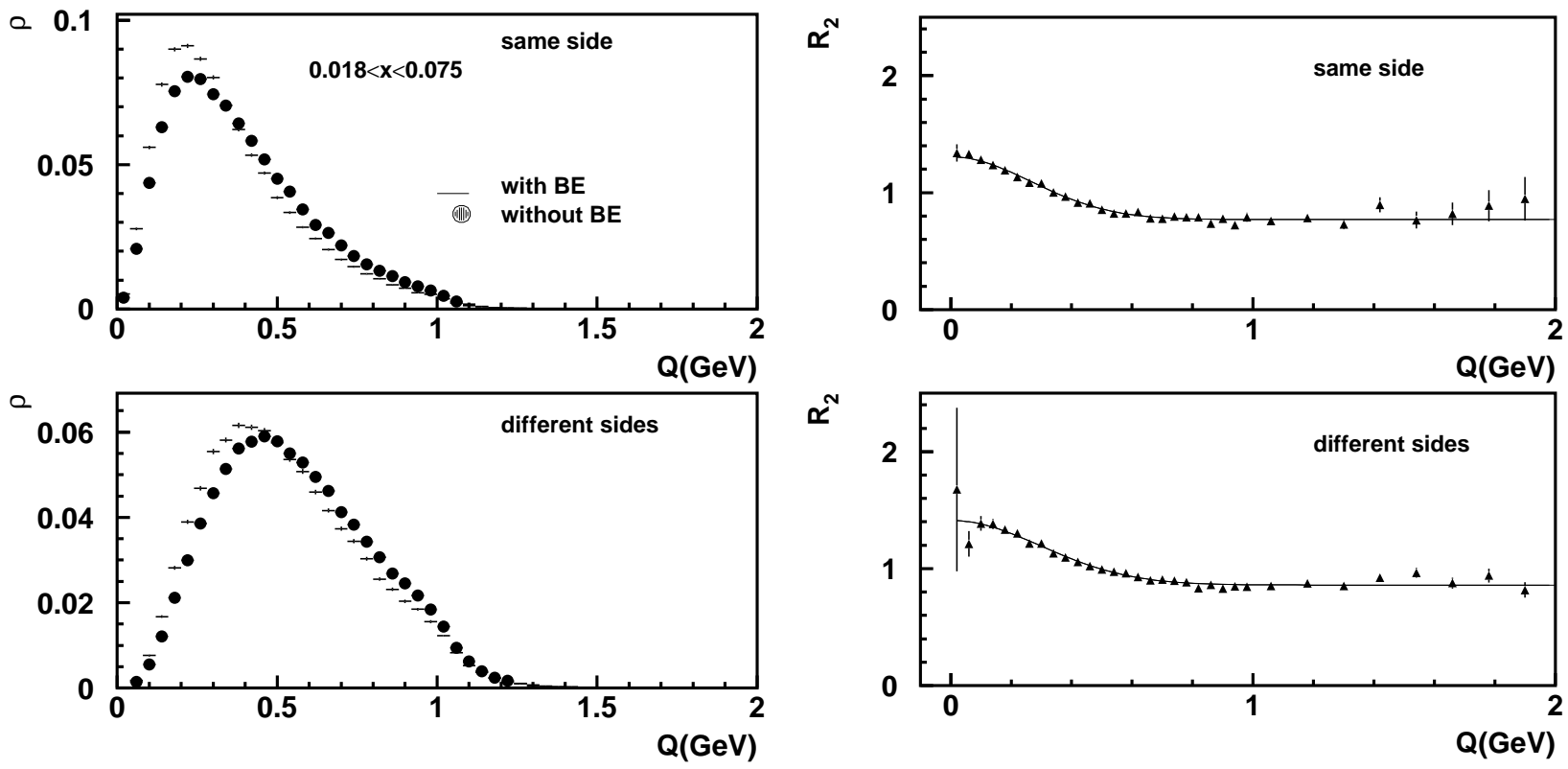

Figure 7.6: Normalized $Q$ and $R_{2}$ distributions as in Fig. 7.5 except only particles in the region of $0.018<x<0.075$ are used. The fitted line is shown to guide the eye. 

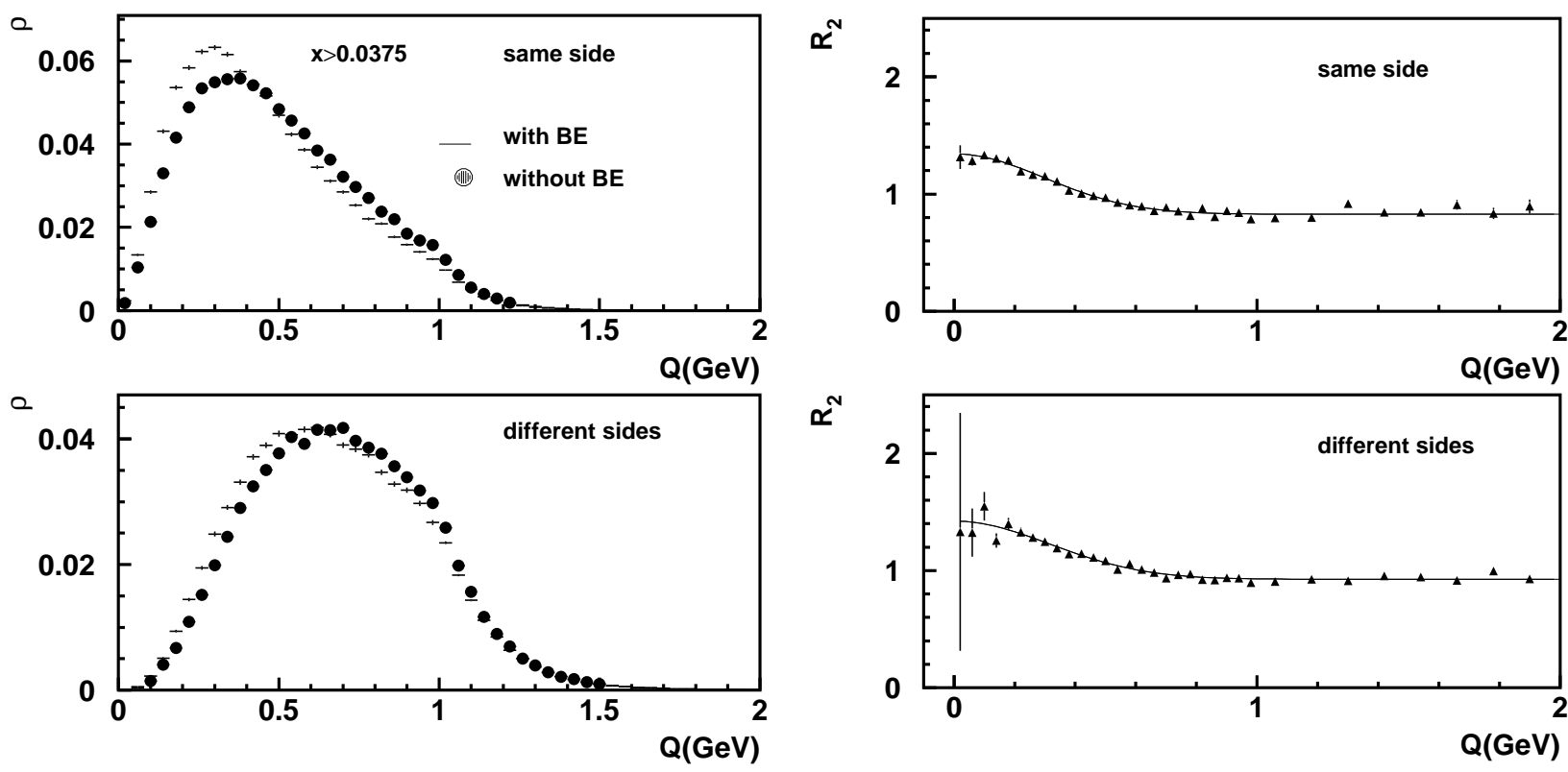

Figure 7.7: Normalized $Q$ and $R_{2}$ distributions as in Fig. 7.5 except only particles in the region of $0.018<x<0.075$ are used. The fitted line is shown to guide the eye.
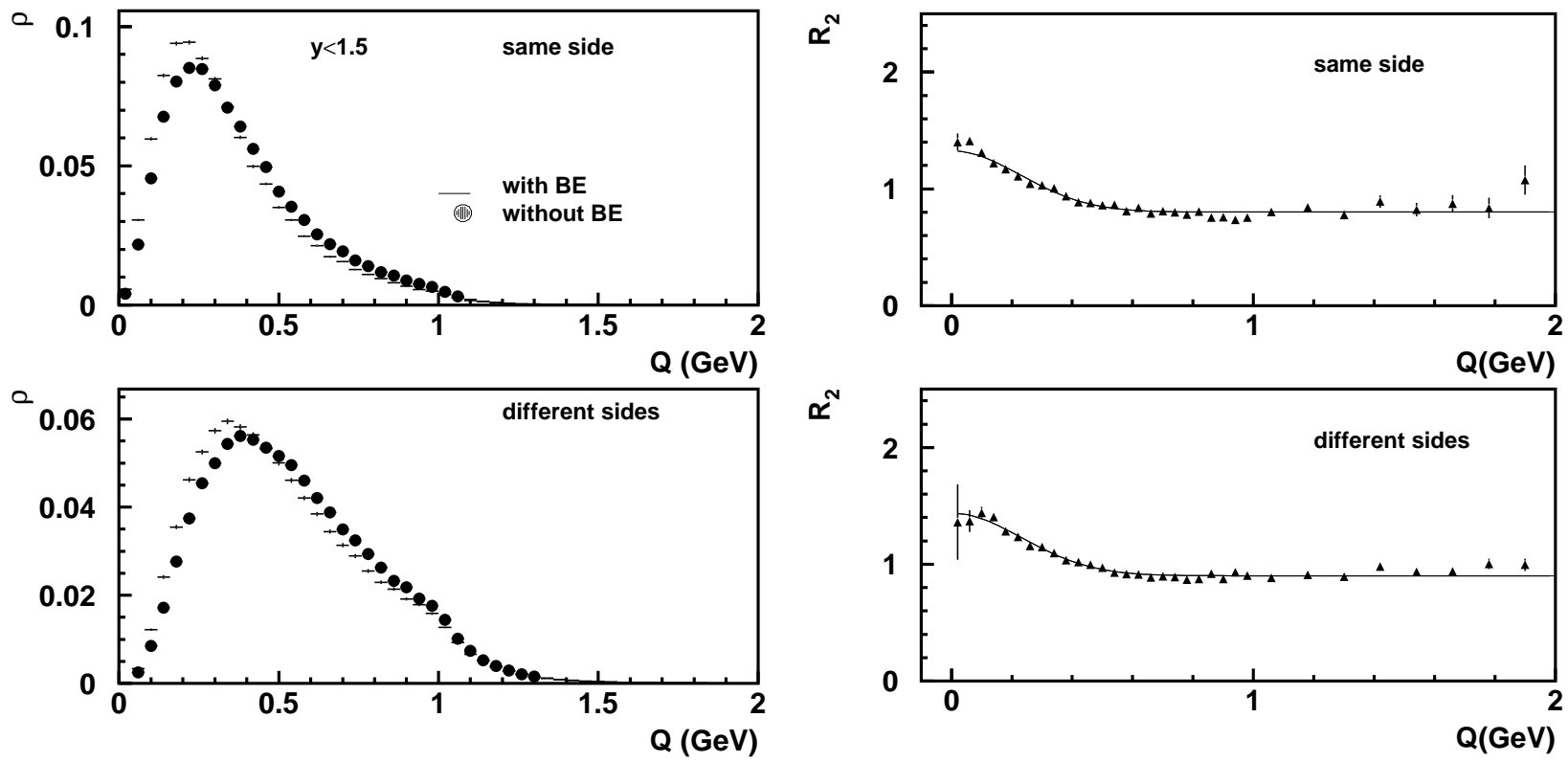

Figure 7.8: Normalized $Q$ and $R_{2}$ distributions as in Fig. 7.5 except only particles in the region of $0.018<x<0.075$ are used. The fitted line is shown to guide the eye. 

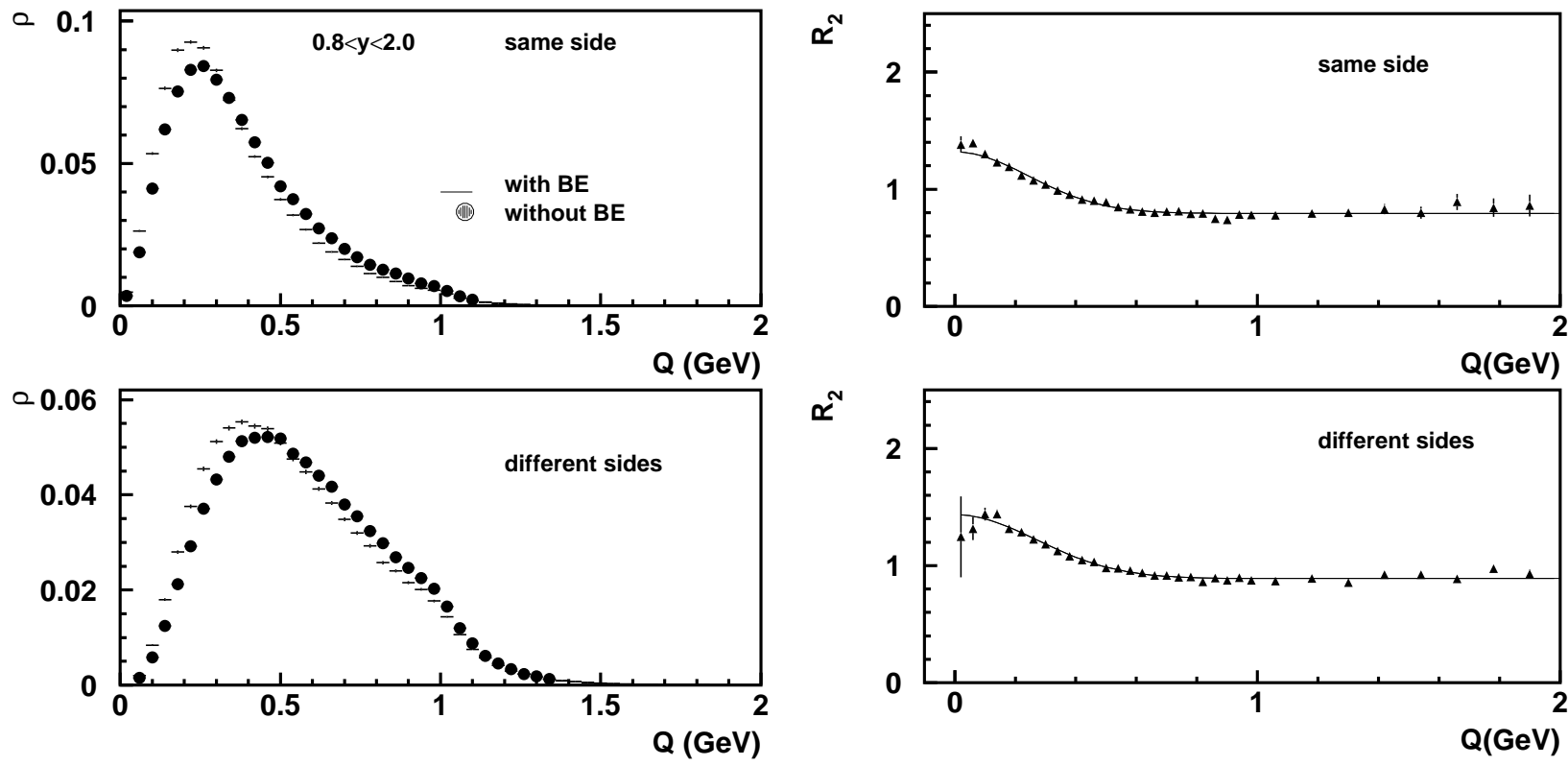

Figure 7.9: Normalized $Q$ and $R_{2}$ distributions as in Fig. 7.5 except only particles in the region of $0.018<x<0.075$ are used. The fitted line is shown to guide the eye.
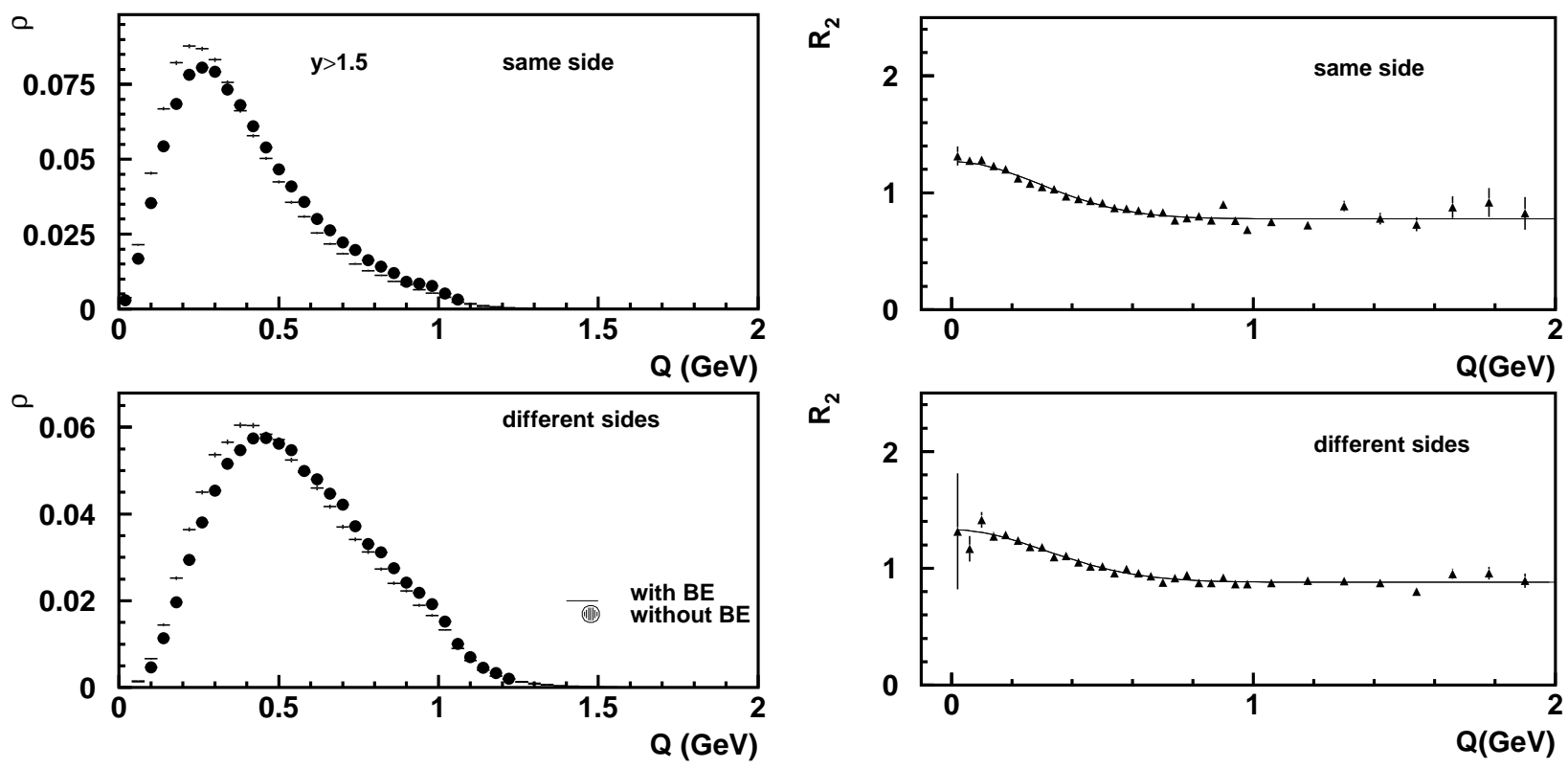

Figure 7.10: Normalized $Q$ and $R_{2}$ distributions as in Fig. 7.5 except only particles in the region of $0.018<x<0.075$ are used. The fitted line is shown to guide the eye. 
$Z$ axis. A new frame is set up as described at the beginning of this chapter. The particles are reassigned to the new jet directions.

The particles are required to have an angle to the gluon jet larger than $4.8^{\circ}, 3.0^{\circ}$ or $2.0^{\circ}$, respectively, according to the energy of the gluon jet from which the particles originate: 8.5$15 \mathrm{GeV}, 15-25 \mathrm{GeV}$ and $25-45.6 \mathrm{GeV}$, thus taking into account the angular resolution of the jet directions as studied in Sect. 7.1.2.

Particles are required to have an angle to the $Z$ axis in the event plane less than $10^{\circ}$ to take into account the width of the gluon jet as studied in Sect. 7.1.1.

If the particle is far away from the event plane, or even perpendicular to the plane, the ambiguity as to which side of gluon it belongs is large. Therefore, the angle between the particle and the event plane is required to be less than $45^{\circ}$.

$R_{2}$ is calculated using Eq. (5.4). The comparison is done for all 3-jet events, and in special configurations of 3-jet events: Yq events, Mercedes events and Yg events.

\subsubsection{Gluon Jets in All 3-jet Events}

Gluon jets are studied in light-quark 3-jet events with the event discriminant $\delta_{\text {event }}<1.3$ and in the heavy-quark 3-jet events with $\delta_{\text {event }}>2.5$ and jet discriminant $\delta_{\text {jet }}=0.8$. The purity of the gluon jets is $68 \%$ in light-quark events and $84 \%$ in heavy-quark events.

The $R_{2}$ distributions are shown in Fig. 7.11. Because of low statistics of particle pairs from the same and from different sides of the gluon, the fitted $\lambda$ and $R$ values have large statistical errors. Instead of comparing $\lambda$ or $R$ from same side with different sides, we therefore study the $R_{2}$ difference $\Delta R_{2}=R_{2}$ (same) $-R_{2}$ (diff). It is shown in the lower row of Fig. 7.11 for lightquark events (left) and heavy-quark events (right). $\Delta R_{2}$ is close to zero and has no significant $Q$ dependence, either for light-quark or for heavy-quark events.

A further check is performed on whether $R_{2}$ of pairs from the same side and different sides is influenced by the detector correction term $C_{\mathrm{det}}$.

Fig. 7.12 shows the detector correction term $C_{\text {det }}$ as a function of $Q$ in the light-quark and heavy-quark events. It can be seen that $C_{\mathrm{det}}$ has no particular structure at low $Q$, but varies smoothly and approximately linearly with $Q$ for both same side and different sides. Hence, the difference in $R_{2}$ for same-side and different-side pairs in the low- $Q$ area can not be caused by $C_{\text {det }}$.

\subsubsection{Gluon Jets in Yq events}

In the light-quark Yq events gluon jets are selected using energy ranking, in the heavy-quark Yq events using double b-tagging. After the selection, 42541 gluon jets from the light-quark events are selected with purity $54 \%$ and efficiency $18 \%$, and 4191 gluon jets from the heavy-quark events are selected with purity $75 \%$ and efficiency $17 \%$.

Fig. 7.13 shows the $R_{2}$ distribution using particle pairs from the same side and different sides of gluon jets in the Yq events. In order to see more clearly the correlation strength difference between $R_{2}$ by using pairs from the same side and different sides of the gluon jet, $\Delta R_{2}$ is shown in Fig. 7.13 as well. 

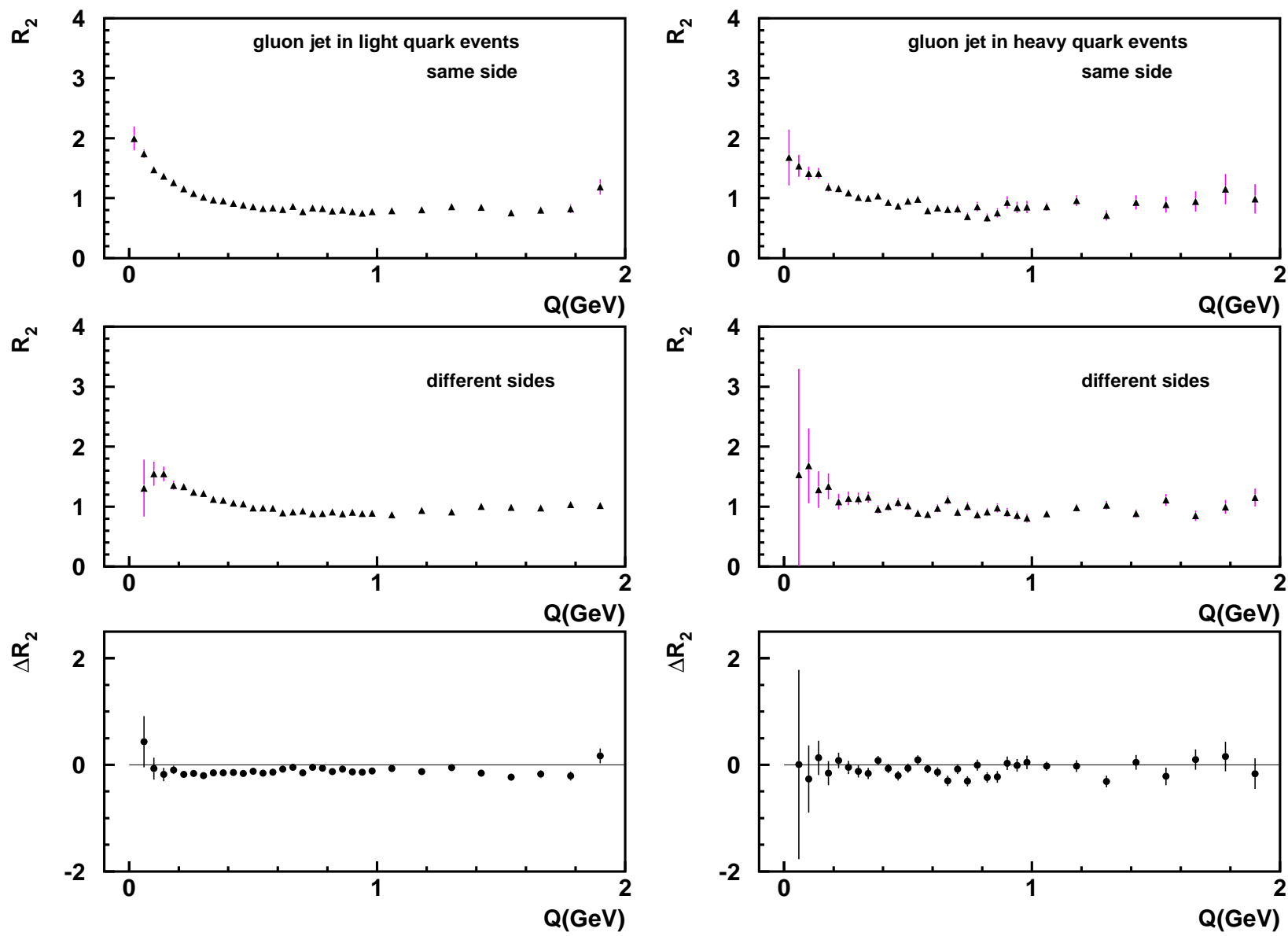

Figure 7.11: $R_{2}$ distribution using particle pairs from the same side (upper plots) and different sides (middle) of the gluon jet. The $\Delta R_{2}$ distribution in the light-quark events (lower left) and in the heavy-quark events (lower right). The left three plots are for gluon jets in the light-quark events and the right three plots are for heavy-quark events. JETSET without BEC is used as the reference sample.
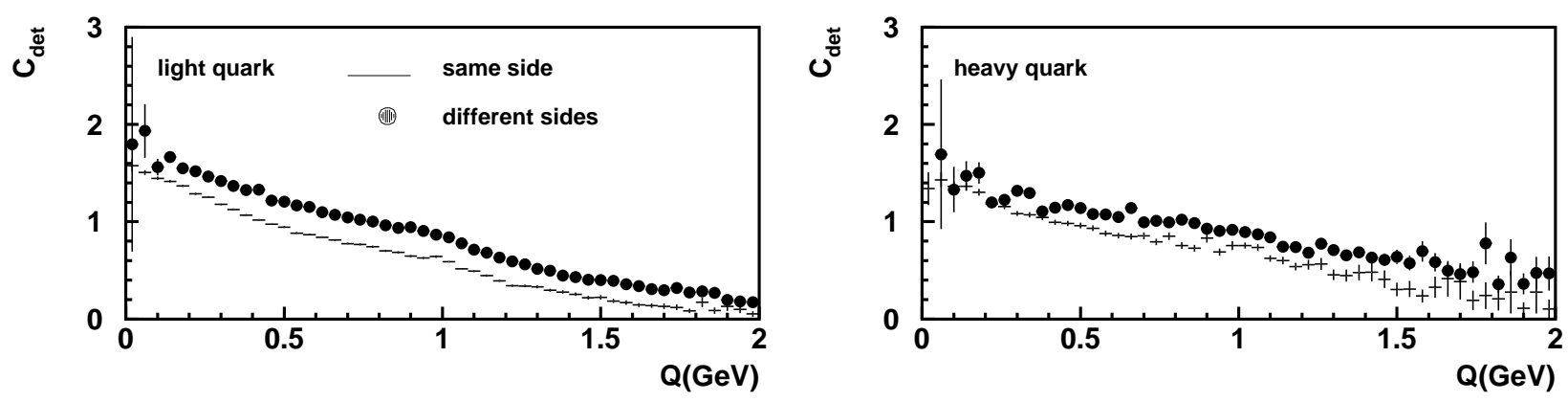

Figure 7.12: The detector correction factor $C_{\mathrm{det}}$ as a function of $Q$ in the light-quark events (left), and in the heavy-quark events (right) using JETSET with BEC. Particle pairs from the same side (histograms) and different sides (dots) of the gluon are used. 
It can be seen from Fig. 7.13 that for both light-quark and heavy-quark events, $\Delta R_{2}$ is close to zero and has no significant dependence on $Q$, which means there is no significant difference between the same side and different sides of the gluon jet.
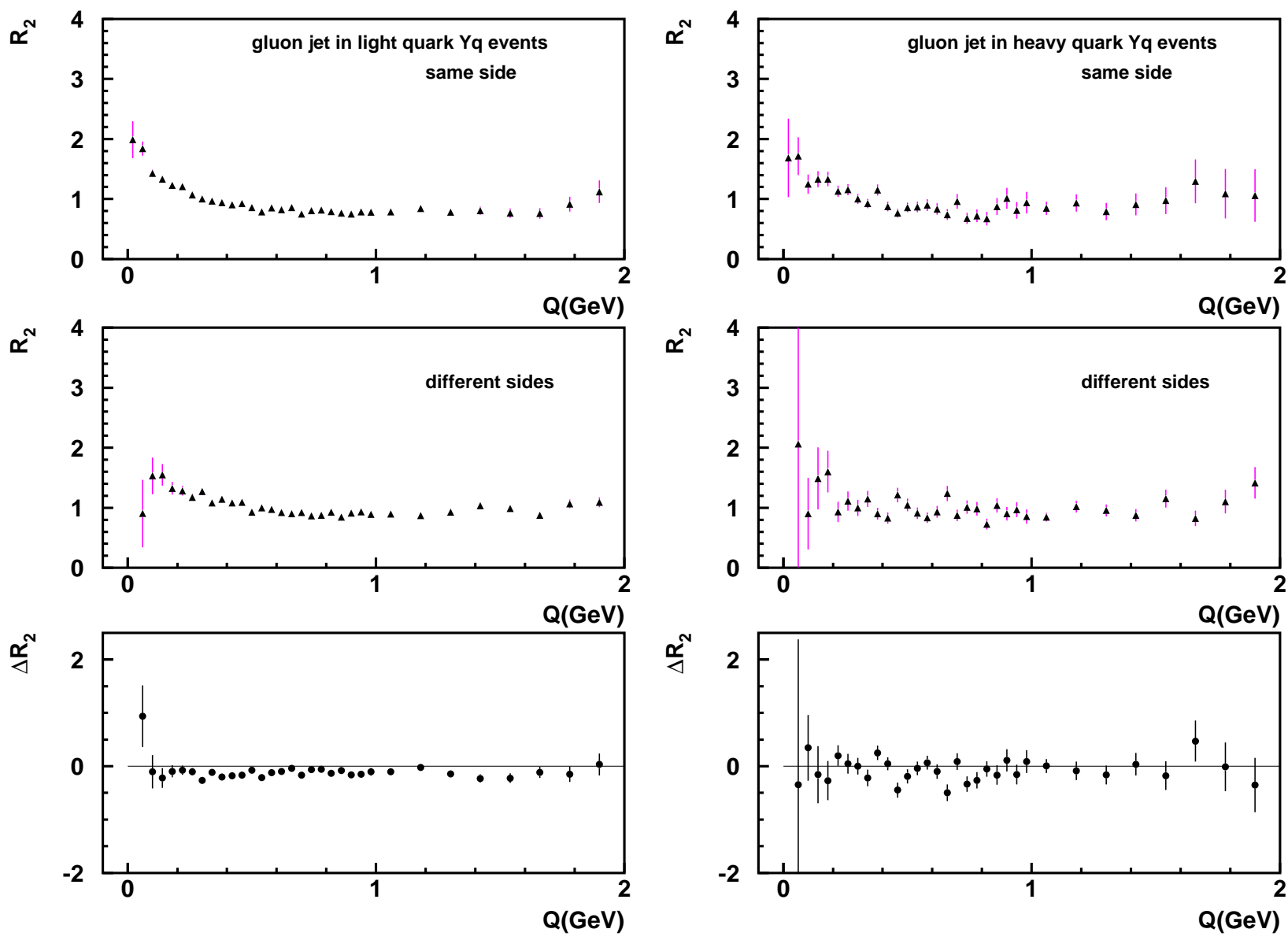

Figure 7.13: $R_{2}$ and $\Delta R_{2}$ distributions using particle pairs from the same side (upper plots) and different sides (middle) of gluon jets in Yq events. The left three plots are for gluon jets in lightquark events and the right three plots are for heavy-quark events. JETSET without BEC is used as the reference sample.

\subsubsection{Gluon Jets in Mercedes Events}

In the light-quark Mercedes events, gluon jets are obtained according to energy ranking, in the heavy-quark Mercedes events as based on double b-tagging. After the selection, 6496 gluon jets make up the light-quark Mercedes sample with purity $39 \%$ and efficiency $2.5 \%$, while 552 gluon jets are found in the heavy-quark Mercedes sample with purity $74 \%$ and efficiency $2.5 \%$. Even though the purity is low for the light-quark sample, we show the result for completeness.

Fig. 7.14 shows the $R_{2}$ and $\Delta R_{2}$ distributions using particle pairs from the same side and from different sides of the gluon jet. Several points in the low- $Q$ area of the $R_{2}$ distributions using particle pairs from different sides of the gluon jet are zero. As seen at generator level in Sect. 7.1.3, 
the number of pairs at low $Q$ is lower for different-side pairs than for same-side pairs. This effect is more pronounced here than at generator level (Sect. 7.1.3) due to the extra requirement that particles have an angle greater than $3.4^{\circ}$ on average with the gluon jet axis. Therefore $\Delta R_{2}$ can not be calculated for these points.

It can be seen that for both light-quark and heavy-quark Mercedes events $\Delta R_{2}$ is close to zero and has no significant $Q$ dependence. There is no significant difference between particle pairs from the same side and from different sides of the gluon.
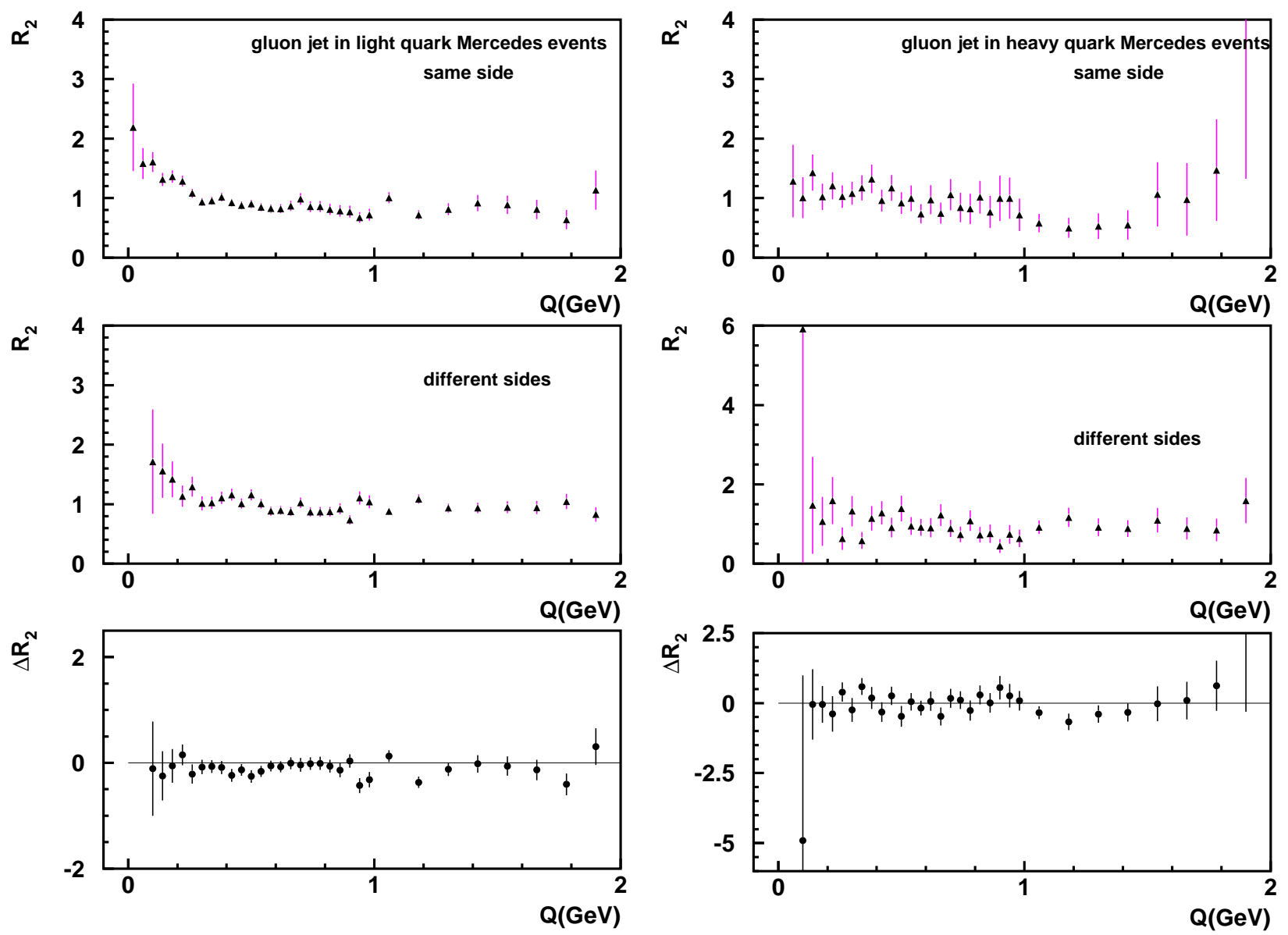

Figure 7.14: $R_{2}$ and $\Delta R_{2}$ distributions using particle pairs from the same side (upper plots) and different sides (middle) of gluon jets in Mercedes events. The left three plots are for gluon jets in the light-quark events and the right three plots are for heavy-quark events. JETSET without BEC is used as the reference sample.

\subsubsection{Gluon Jets in Yg Events}

Double b-tagging is used to select the gluon jet in heavy-quark events, with the event and jet discriminant $\delta_{\text {event }}=2.5$ and $\delta_{\text {jet }}=0.8$. After the selection, 939 gluon jets remain with a purity and efficiency of $32 \%$ and $2 \%$, respectively.

Fig. 7.15 shows the $R_{2}$ and $\Delta R_{2}$ distributions for pairs of particles from the same side and 

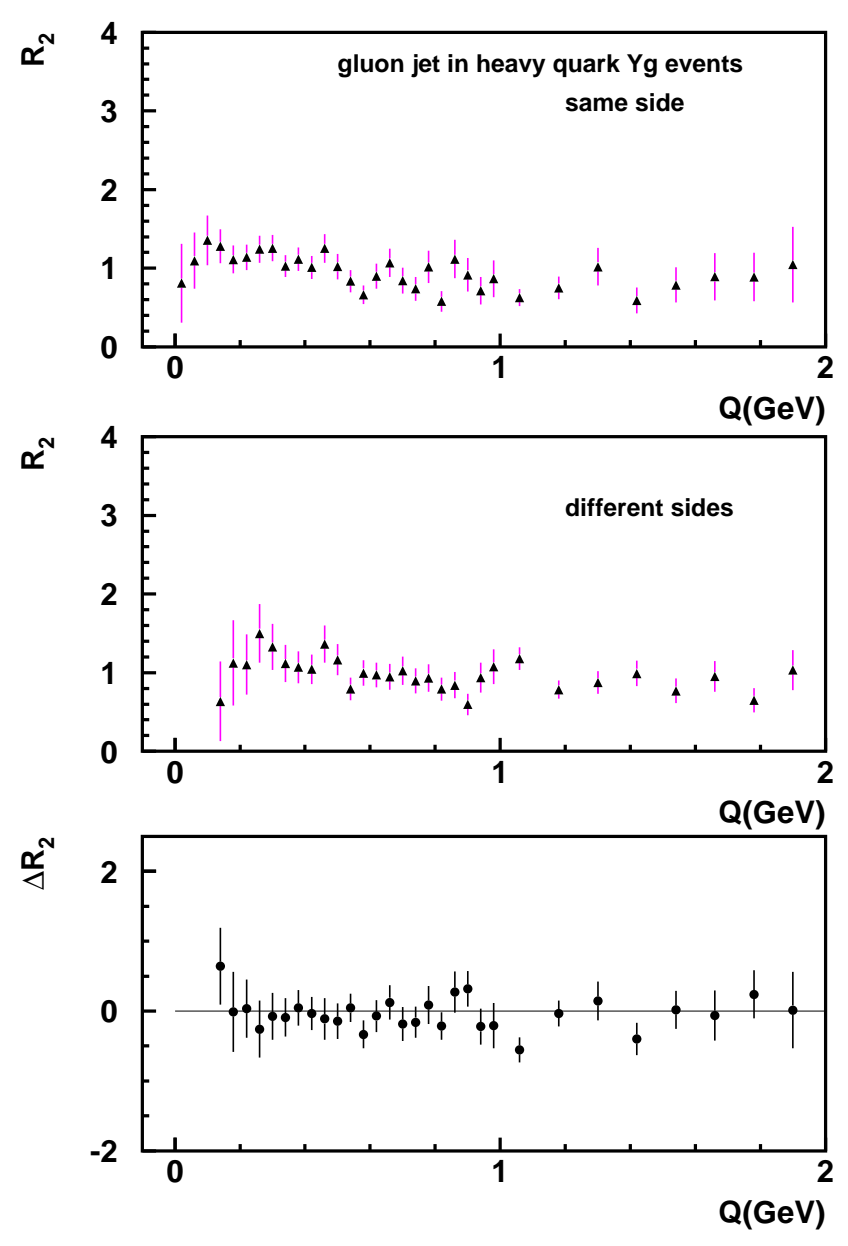

Figure 7.15: $R_{2}$ and $\Delta R_{2}$ distributions using particle pairs from the same side (upper) and different sides (middle) of the gluon jet in Yg events. JETSET without BEC is used as the reference sample.

from different sides of the gluon jet in the heavy-quark Yg events. Just as in the Mercedes events, several points in the low- $Q$ area of the $\Delta R_{2}$ distribution are zero for different-side pairs, and those points are not included in the $\Delta R_{2}$ plot. It can be seen that $\Delta R_{2}$ is consistent with zero and has no significant $Q$ dependence.

\subsection{Rapidity and $x$ Dependence of the Differences}

When comparing $R_{2}$ for particle pairs from the same side and from different sides of gluon jets in all 3-jet events, Yq events, Mercedes events and Yg events, we noticed that $\Delta R_{2}$ is zero most of the time. Since there is more overlap in the tip of the gluon jets than in the central region, we now compare same-side pairs and different-side pairs in different windows of $x$ and rapidity $y$, where $x$ and $y$ are as defined in (6.2) and (6.3). We expect a difference, if present, to increase with increasing $x$ and $y$. 


\subsubsection{Gluon Jets in All 3-jet Events}

The particle pairs used to calculate $R_{2}$ are from the gluon jets in the light-quark and heavy-quark 3 -jet events.

Fig. 7.16 gives the $R_{2}$ and $\Delta R_{2}$ distributions for same-side and different-side pairs in light-quark 3 -jet events for three $x$ windows. In all three cases no significant difference in correlation strength is seen between the same side and different sides of the gluon.

The corresponding distributions in heavy-quark 3-jet events are shown in Fig. 7.17. The first several points in the low- $Q$ area of the $R_{2}$ distributions using particle pairs from different sides of the gluon jet are zero, and the number of points with $R_{2}$ to be zero increases with increasing $x$, which has already been seen at generator level in Sect. 7.1.3. These points with $R_{2}$ zero are not included in the calculation of $\Delta R_{2}$.

The correlation strength shows no difference when using same side and different sides of the gluon, no matter which part of the gluon jet is used.

Figs. 7.18 and 7.19 show the $R_{2}$ and $\Delta R_{2}$ distributions for light-quark and heavy-quark 3-jet events, respectively, in three $y$ windows. The conclusion is similar to that for the three $x$ windows. The correlation strength shows no significant dependence on $y$ both for light-quark events and heavy-quark events.

The studies on the different $x$ and $y$ windows of gluon jets in all 3-jet events show similar results in light-quark and heavy-quark events. Moreover, the gluon jet in the heavy-quark events has a higher purity than in light-quark events. Therefore, in the following analysis on Yq events, Mercedes events and Yg events, only heavy-quark events are used.

\subsubsection{Gluon Jets in Yq events}

The $R_{2}$ and $\Delta R_{2}$ distributions for same-side and different-side pairs in heavy-quark Yq events are shown in Fig. 7.20 for three $x$ windows and in Fig. 7.21 for three $y$ windows. In both cases no difference in correlation strength is shown between the same-side and different-side of the gluon jet.

\subsubsection{Gluon Jets in Mercedes Events}

The $R_{2}$ and $\Delta R_{2}$ distributions for same-side and different-side pairs in heavy-quark Mercedes events are shown in Fig. 7.22 for three $x$ windows, and in Fig. 7.23 for three $y$ windows. In all cases no difference in correlation strength is seen between same-side and different-side pairs.

\subsubsection{Gluon Jets in Yg Events}

The $R_{2}$ and $\Delta R_{2}$ distributions for same-side and different-side pairs in heavy-quark Yg events are shown in Fig. 7.24 for three $x$ windows and in Fig. 7.25 for three $y$ windows. The correlation strength of the same-side pairs and different-side pairs is the same within statistical errors. 

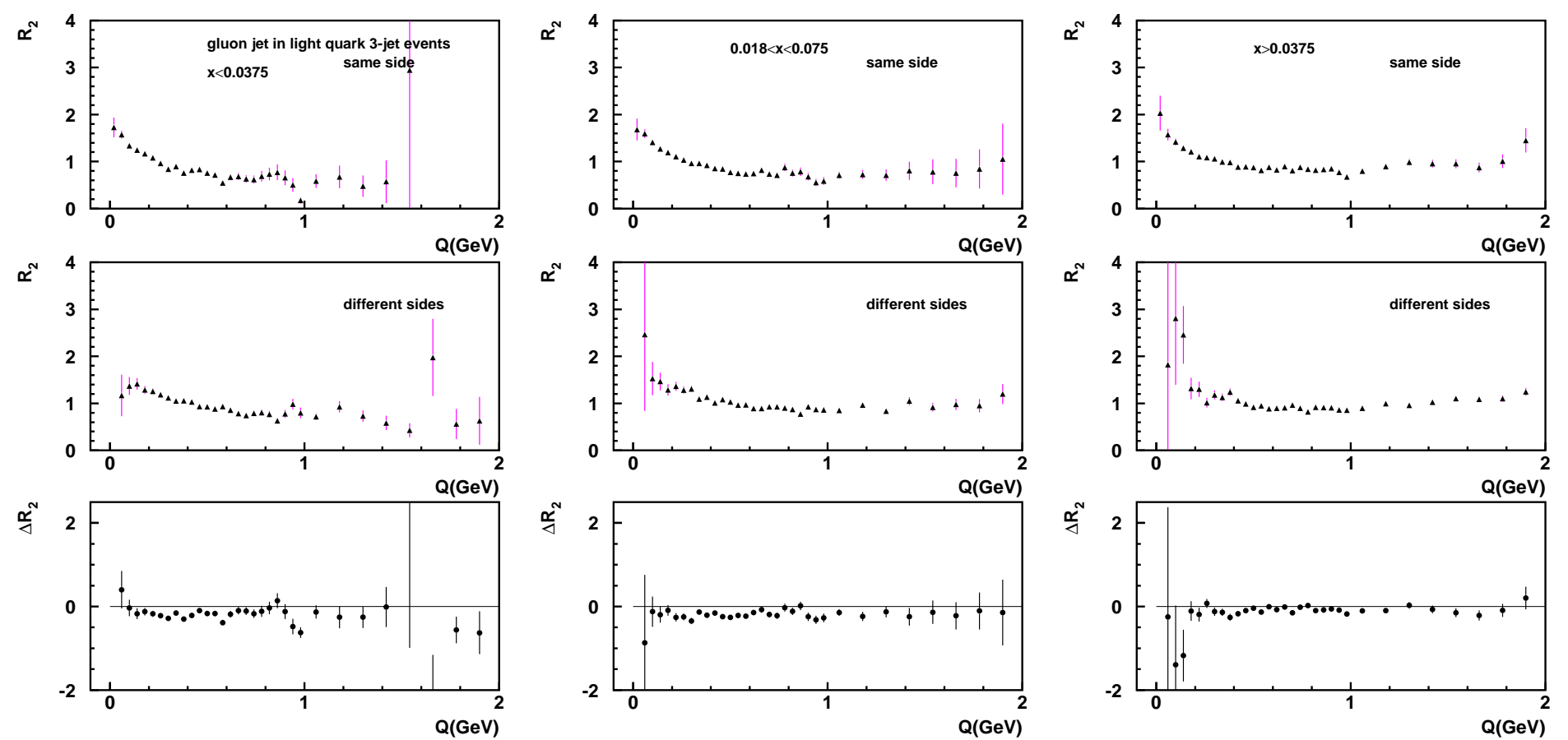

Figure 7.16: $R_{2}$ and $\Delta R_{2}$ distributions using particle pairs from the same side and different sides of gluon jets in the light-quark events for three different $x$ ranges: $x<0.0375,0.018<x<0.075$ and $x>0.0375$. JETSET without BEC is used as the reference sample. 

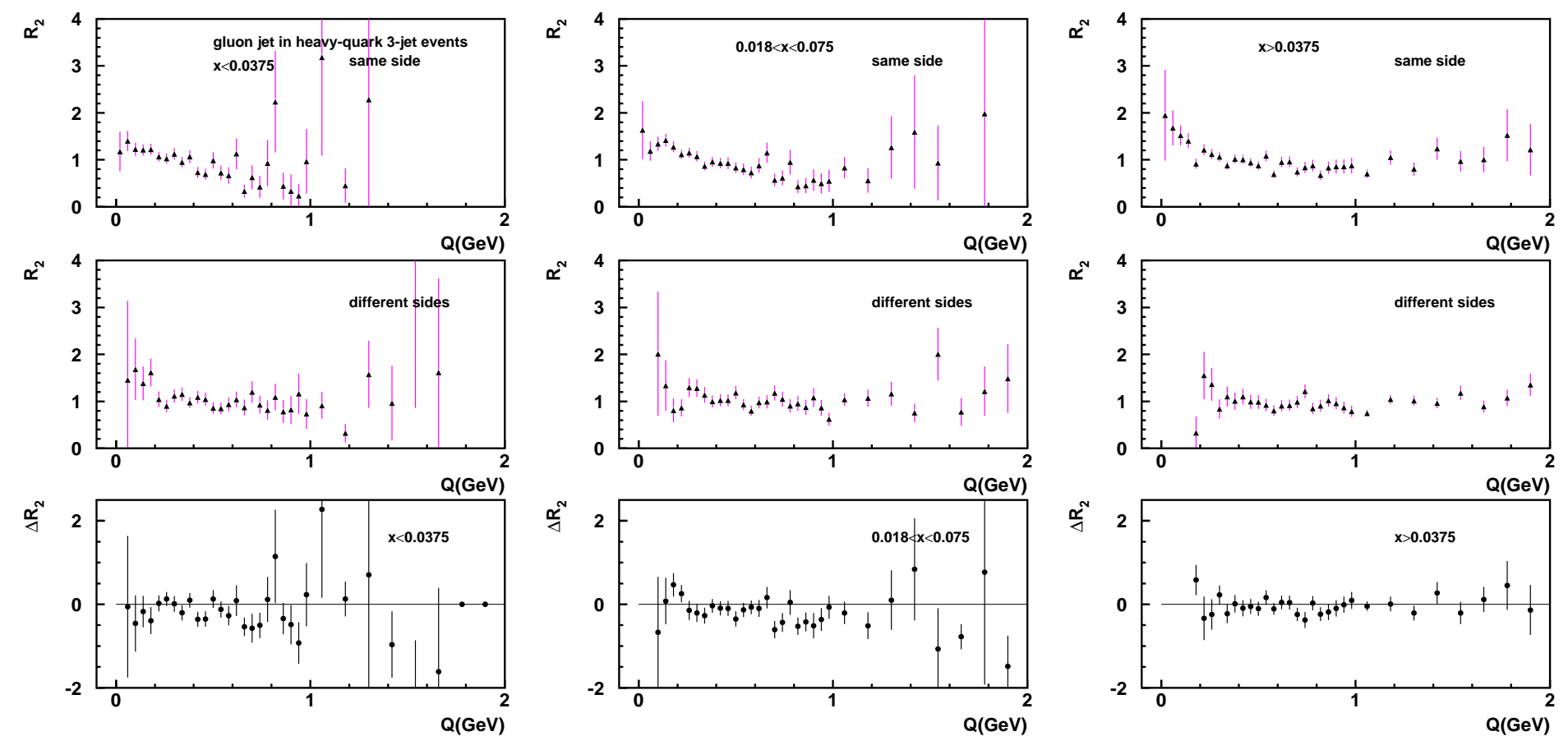

Figure 7.17: $R_{2}$ and $\Delta R_{2}$ distributions using particle pairs from the same side and different sides of gluon jets in the heavy-quark events for three different $x$ ranges: $x<0.0375,0.018<x<0.075$ and $x>0.0375$. JETSET without BEC is used as the reference sample. 

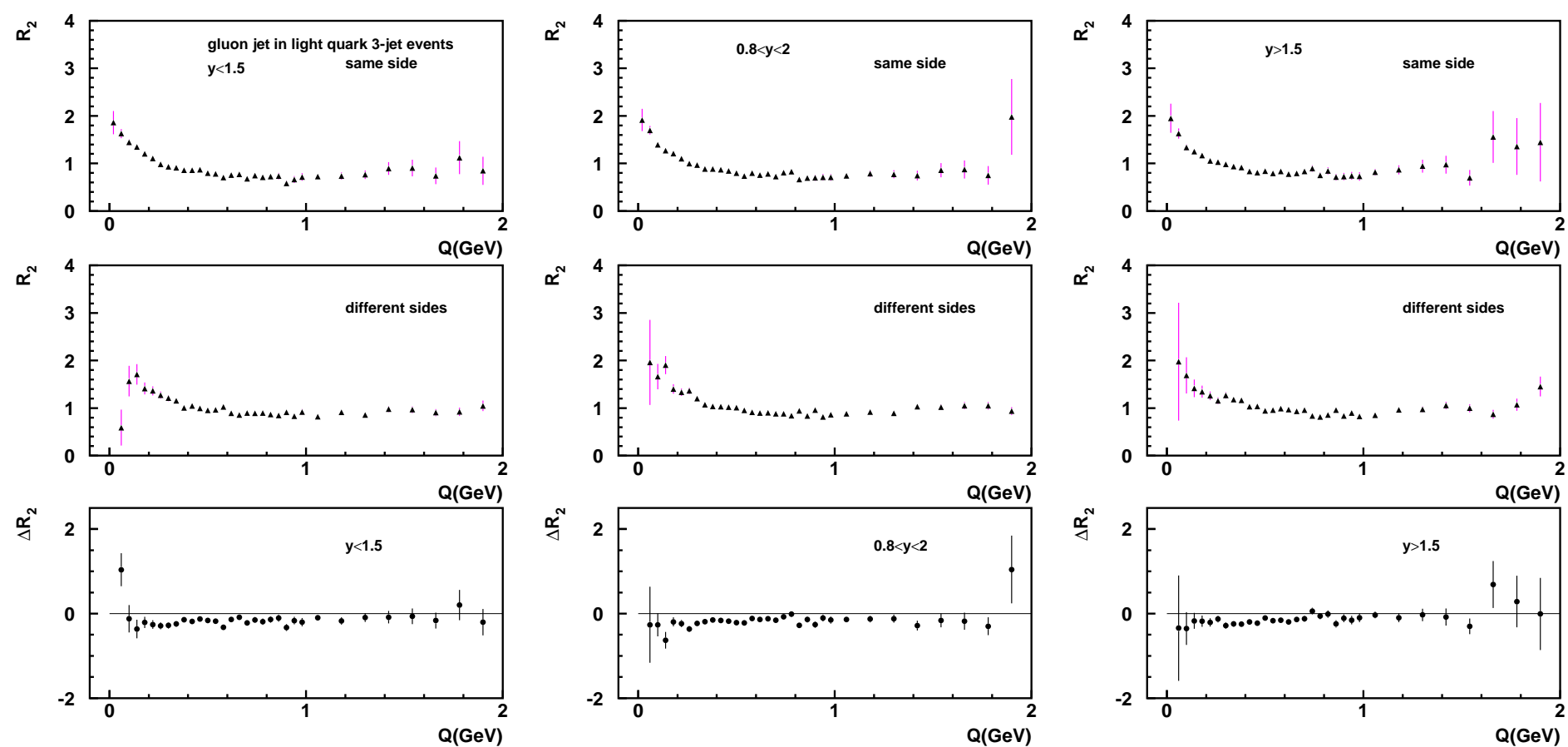

Figure 7.18: $R_{2}$ and $\Delta R_{2}$ distributions using particle pairs from the same side and different sides of gluon jets in the light-quark events for three different $y$ ranges: $y<1.5,0.8<y<2$ and $y>1.5$. JETSET without BEC is used as the reference sample. 

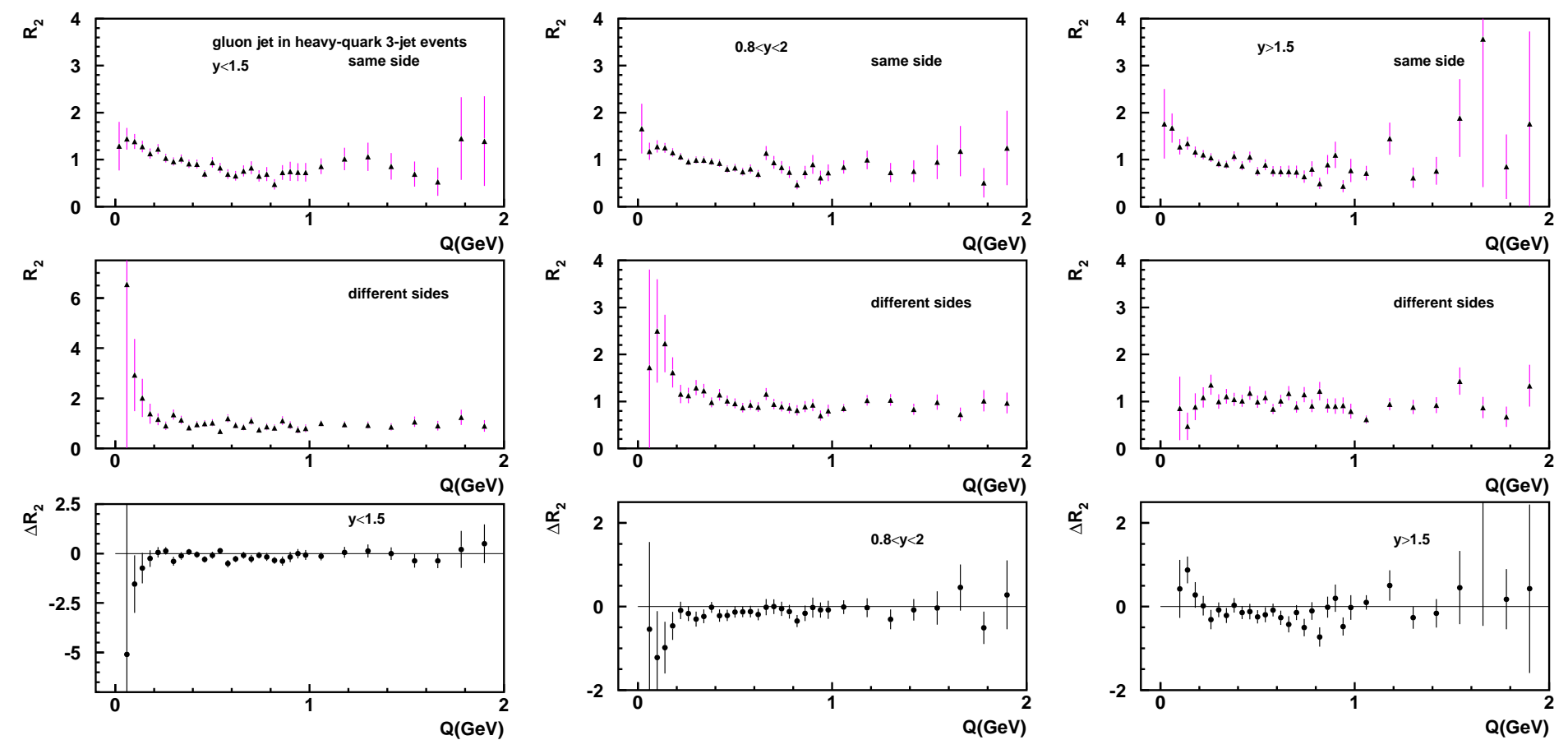

Figure 7.19: $R_{2}$ and $\Delta R_{2}$ distributions using particle pairs from the same side and different sides of gluon jets in the heavy-quark events for three different $y$ ranges: $y<1.5,0.8<y<2$ and $y>1.5$. JETSET without BEC is used as the reference sample. 

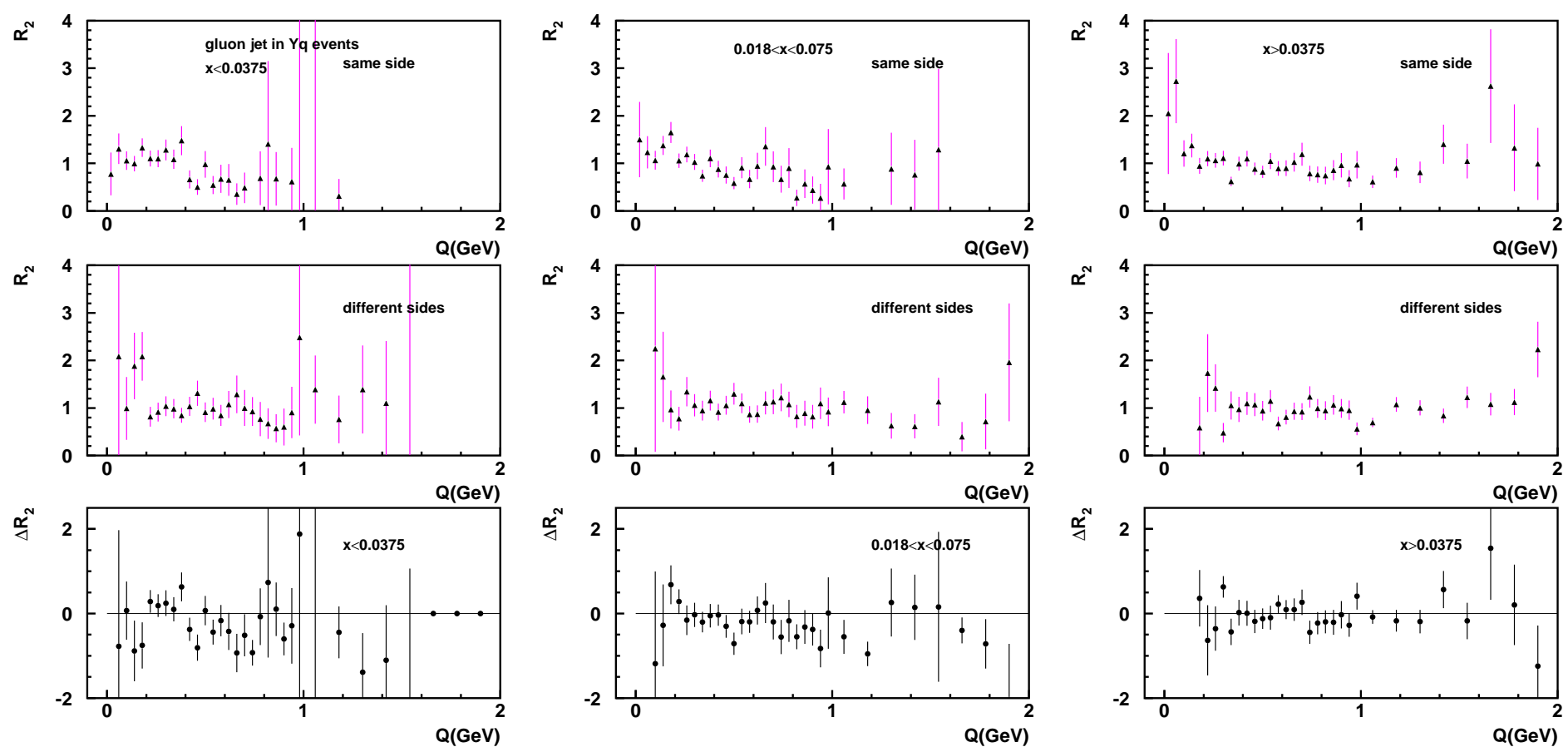

Figure 7.20: $R_{2}$ and $\Delta R_{2}$ distributions using particle pairs from the same side and different sides of gluon jets in the heavy-quark Yq events for three different $x$ ranges: $x<0.0375,0.018<x<0.075$ and $x>0.0375$. JETSET without BEC is used as the reference sample. 

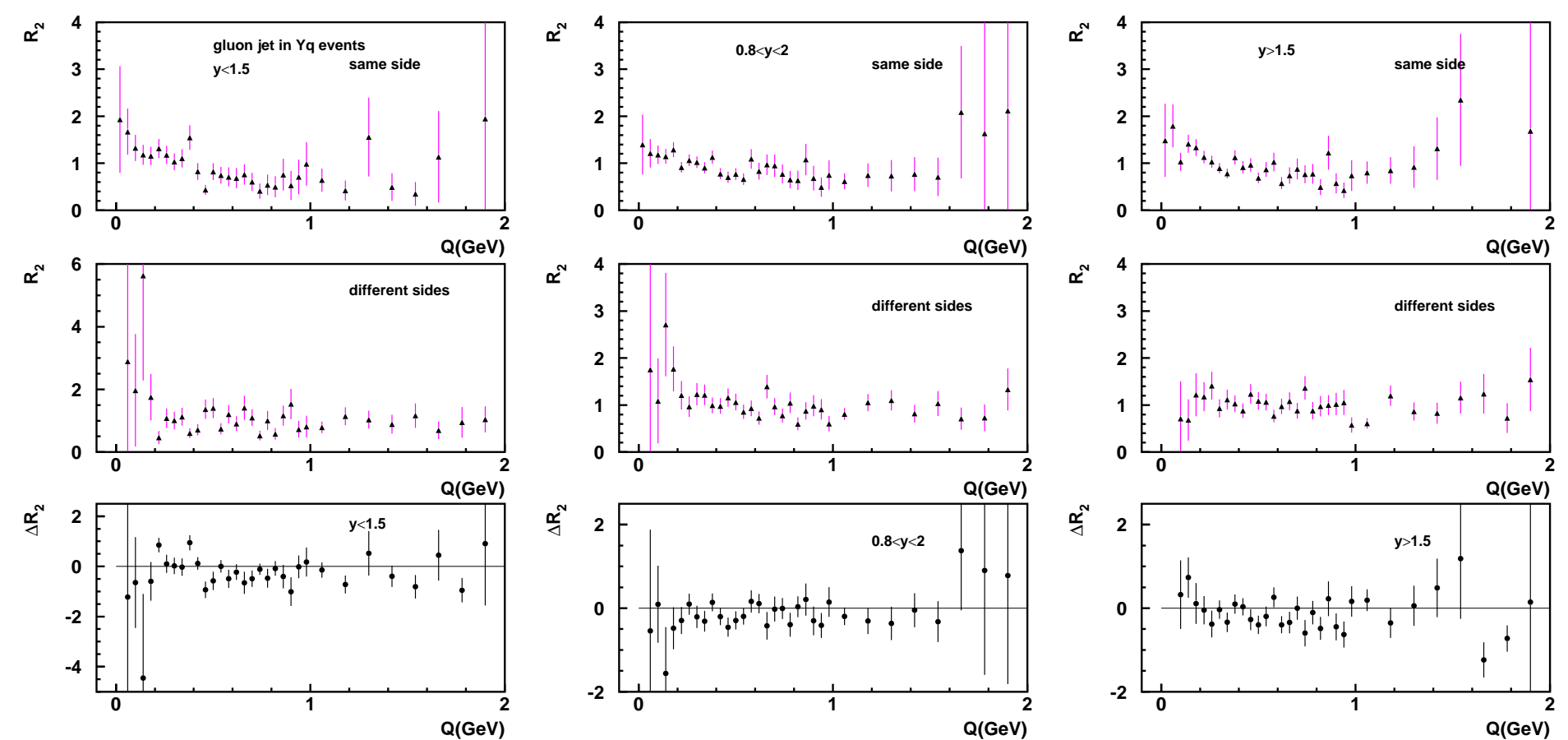

Figure 7.21: $R_{2}$ and $\Delta R_{2}$ distributions using particle pairs from the same side and different sides of gluon jets in the heavy-quark Yq events for three different $y$ ranges: $y<1.5,0.8<y<2$ and $y>1.5$. JETSET without BEC is used as the reference sample. 

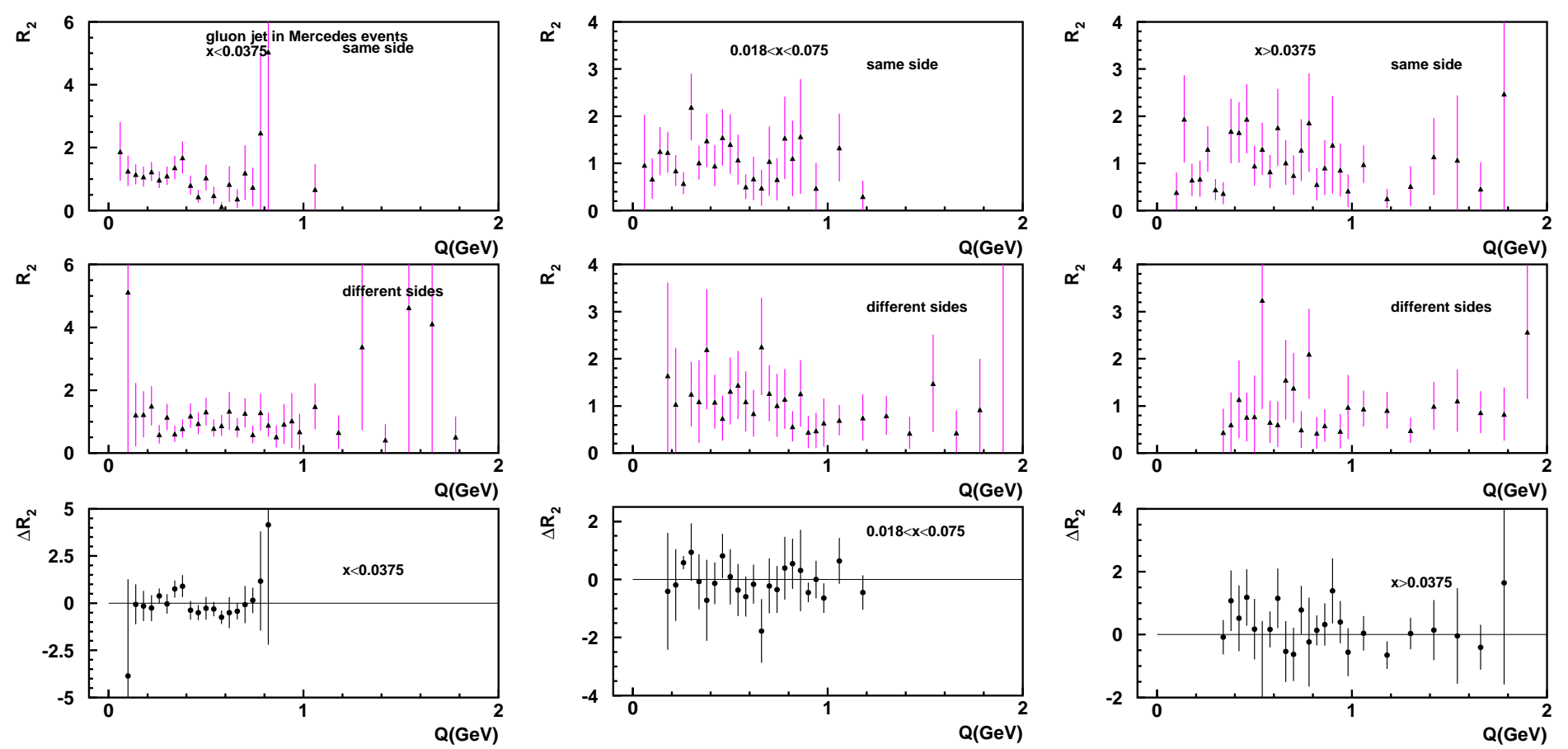

Figure 7.22: $R_{2}$ and $\Delta R_{2}$ distributions using particle pairs from the same side and different sides of gluon jets in the heavy-quark Mercedes events for three different $x$ ranges: $x<0.0375,0.018<x<0.075$ and $x>0.0375$. JETSET without BEC is used as the reference sample. 

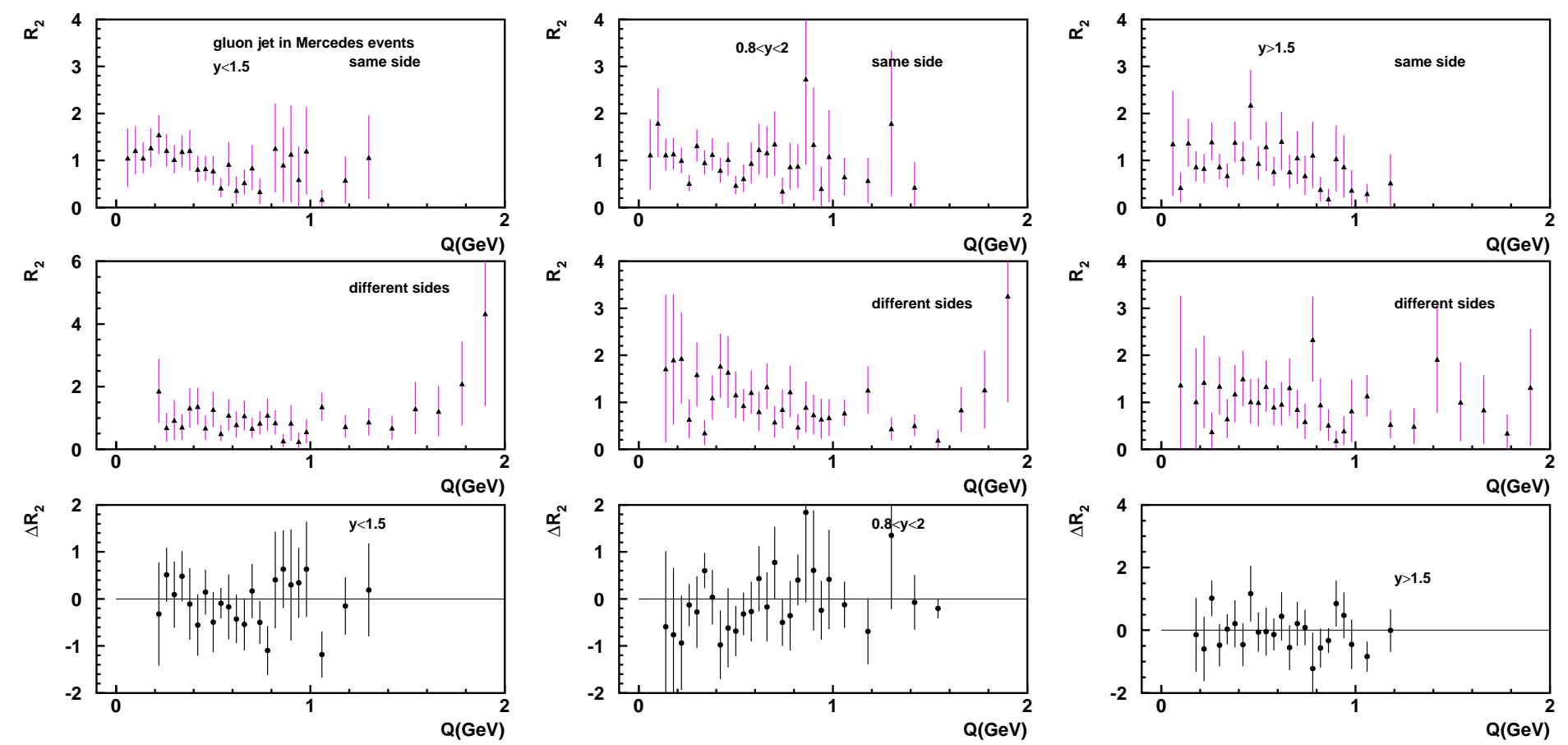

Figure 7.23: $R_{2}$ and $\Delta R_{2}$ distributions using particle pairs from the same side and different sides of gluon jets in the heavy-quark Mercedes events for three different $y$ ranges: $y<1.5,0.8<y<2$ and $y>1.5$. JETSET without BEC is used as the reference sample. 

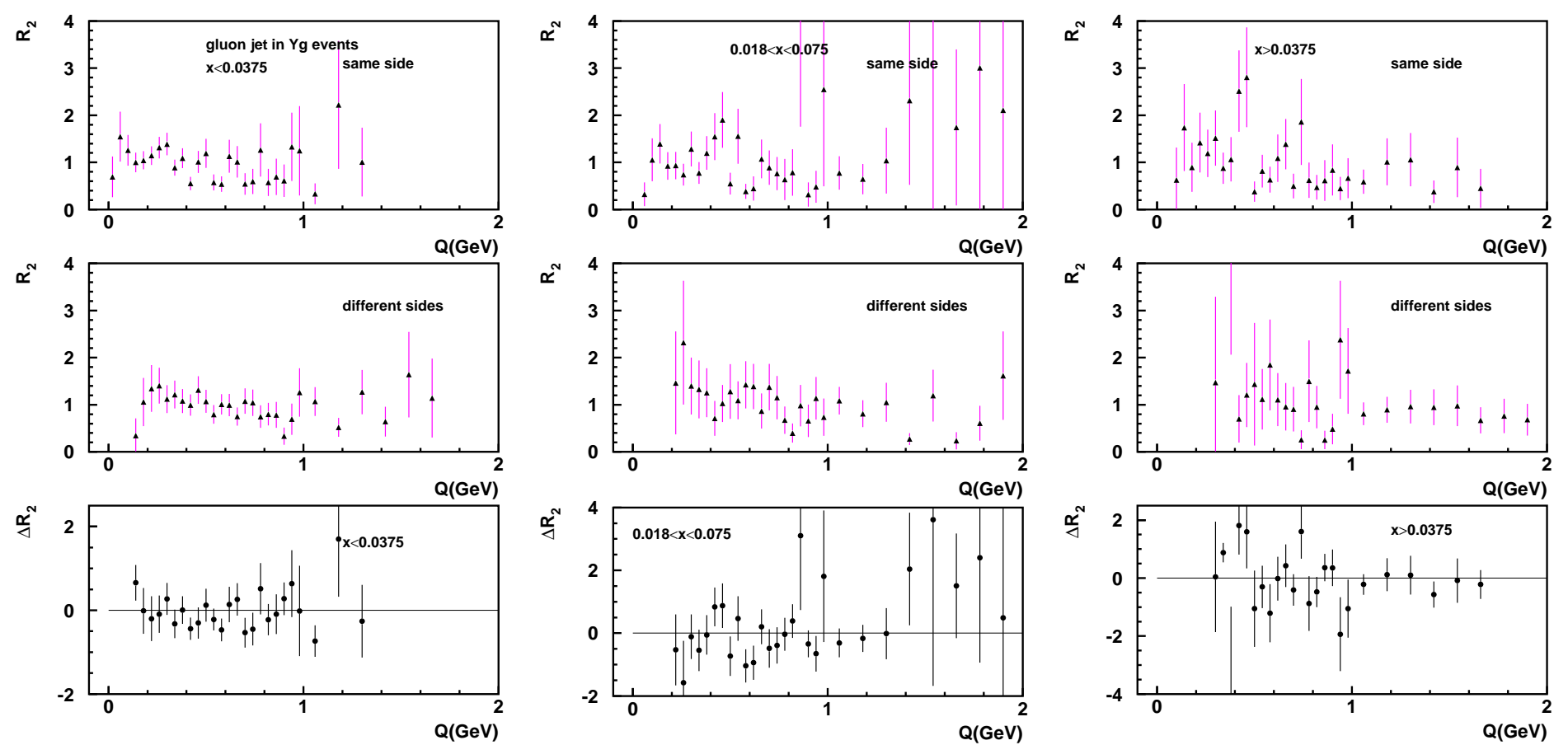

Figure 7.24: $R_{2}$ and $\Delta R_{2}$ distributions using particle pairs from the same side and different sides of gluon jets in the heavy-quark Yg events for three different $x$ ranges: $x<0.0375,0.018<x<0.075$ and $x>0.0375$. JETSET without BEC is used as the reference sample. 

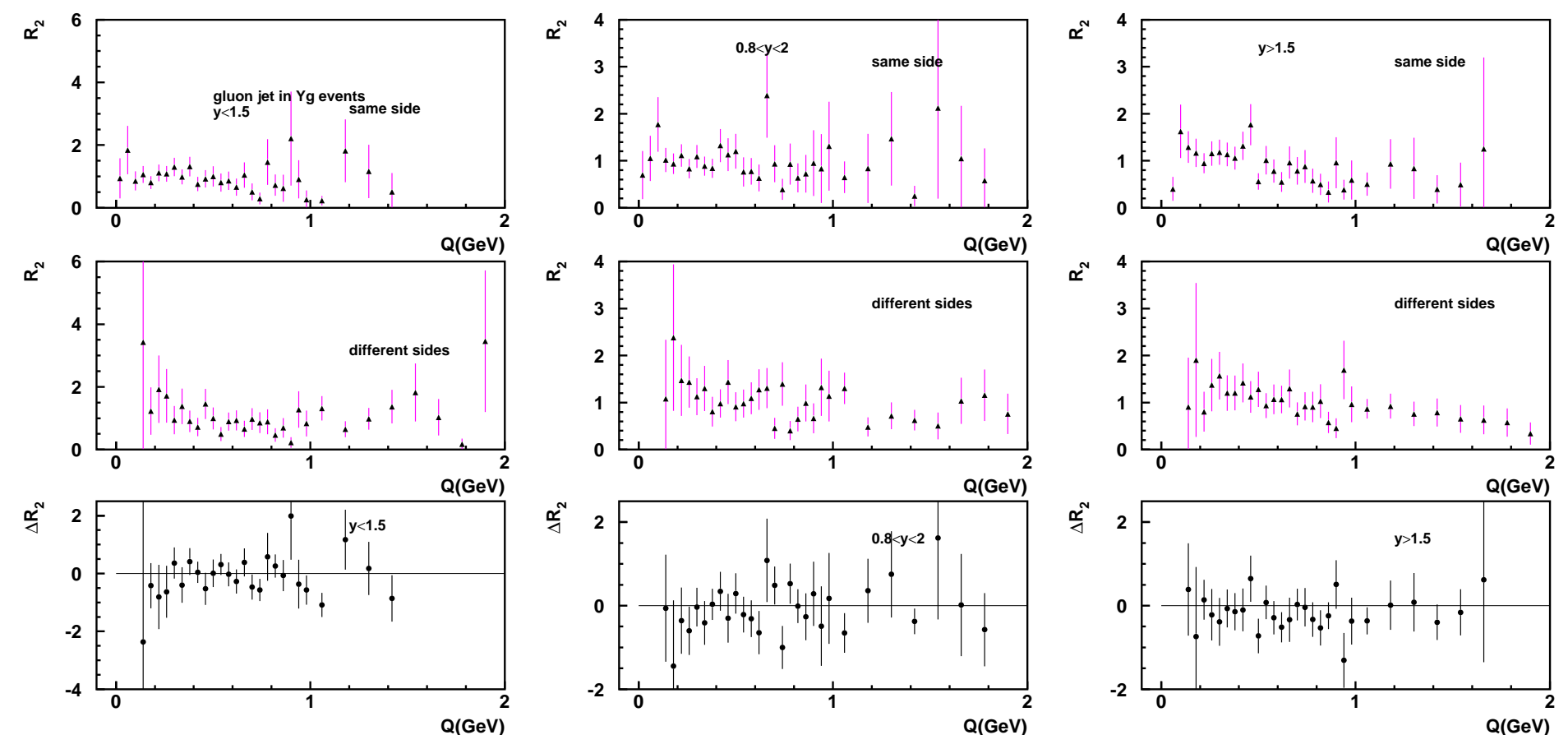

Figure 7.25: $R_{2}$ and $\Delta R_{2}$ distributions using particle pairs from the same side and different sides of gluon jets in the heavy-quark $\mathrm{Yg}$ events for three different $y$ ranges: $y<1.5,0.8<y<2$ and $y>1.5$. JETSET without BEC is used as the reference sample. 


\subsection{Conclusions}

In this chapter, the correlation function $R_{2}$ has been studied by separately using pairs of particles originating from the same side and from different sides of the gluon jet in all three-jet events, $\mathrm{Yq}$ events, Mercedes events and Yg events.

By checking the difference in $R_{2}$, i.e., $\Delta R_{2}$, in the samples of all three-jet events, we see that same-side and different-side pairs do not show much difference, neither in light-quark nor in heavyquark events. Restricting the analysis to Yq events, Mercedes events and Yg events, we do not see difference either from the same-side and different-side pairs with increasing gluon jet energy.

The study of rapidity and $x$ dependence supplies more detailed information on different parts of the gluon jets. However, the tip part of the gluon jet which has more overlap of the strings does not show much difference between the same-side and different-side pairs. 



\section{Chapter 8}

\section{Conclusion}

In this thesis, we have searched for differences in Bose-Einstein correlations between event configurations involving two color strings such as gluon jets and those involving single strings such as quark jets. Comparisons have been made of two-jet and three-jet events, of quark and gluon jets, and of pairs of identical pions originating from the same and from different sides of a gluon jet.

The strength parameter $\lambda$ and the size parameter $R$ of the Bose-Einstein correlation function $R_{2}$ are determined and compared for various configurations. The comparison of two-jet and three-jet events is done for various values of the Durham resolution parameter $y_{\text {cut }}$ and in different multiplicity intervals. Quark and gluon jets are compared for various energy ranges and in various windows of Feynman- $x$ and rapidity $y$. No significant difference is observed between two-jet and three-jet events, or between quark and gluon jets.

Furthermore, $R_{2}$ is compared for pions originating from the same side and from different sides of the gluon jet. Studies are performed in the gluon jet of so-called Yq events, Mercedes events and Yg events, and in different $x$ and $y$ windows, both for light-quark and heavy-quark events. Again, no obvious difference is found between the same-side and the different-side pairs.

Referring back to our original motivation quoted in Sect. 1.3, at the given level of statistical significance, our results support our options (2) of the presence of Bose-Einstein correlations in pairs of pions originating from two different sources when these two sources are overlapping, or (3) of the lack of any overlap. 



\section{References}

[1] W. Pauli, Phys. Rev. 58 (1940) 716.

[2] R. Hanbury Brown and R. Q. Twiss, Nature 58 (1957) 716.

[3] G. Goldhaber, S. Goldhaber, W. Lee and A. Pais, Phys. Rev. 120 (1960) 300.

[4] G. Goldhaber et al., Phys. Rev. Lett. 3 (1959) 181.

[5] G. N. Fowler and R. M. Weiner, Phys. Lett. 70B (1977) 201.

[6] G. N. Fowler, N. Stelte and R. M. Weiner, Nucl. Phys. A319 (1979) 349.

[7] R. M. Weiner, Introduction to Bose-Einstein Correlations and Subatomic Interferometry, John Wiley \& Sons Ltd, England. (2000).

[8] M. Deutschmann et al., Nucl. Phys. B204 (1982) 333.

[9] Jorn van Dalen, Ph.D. thesis, University of Nijmegen (2002).

[10] M. G. Bowler, Z. Phys. C46 (1990) 305.

[11] M. Suzuki, Phys. Rev. D35 (1987) 3359.

[12] M. G. Bowler, Z. Phys. C39 (1988) 81.

[13] G. Marchesini and B. R. Webber, Nucl. Phys. B238 (1984) 1.

[14] B. Andersson, G. Gustafson, G. Ingelman and T. Sjöstrand, Phys. Rep. 97 (1983) 31.

[15] B. Andersson and W. Hofmann, Phys. Lett. B169 (1986) 364.

[16] B. Andersson, The Lund Model, Cambridge University Press, Cambridge (1998).

[17] B. Andersson, B. and M. Ringnér, Nucl. Phys. B513 (1998) 627.

[18] B. Andersson, B. and M. Ringnér, Phys. Lett. B421 (1998) 283.

[19] J. Häkkinen and M. Ringnér, Eur. Phys. J. C5 (1998) 275.

[20] LEP Electroweak Working Group, A Combination of Preliminary Electroweak Measurements and Constraints on the Standard Model, LEPEWWG/2005-01, Eprint hep-ex/0511027. 
[21] P. Lipa and B. Buschbeck, Phys. Lett. B223 (1989) 465.

[22] B. Buschbeck, H. C. Eggers and P. Lipa, Phys. Lett. B481 (2000) 187.

[23] N. van Remortel, Ph.D. thesis, University of Antwerpen (2003).

[24] T. Csörgő, S. Hegyi and W. A. Zajc, Eur. Phys. J. C36 (2004) 67.

[25] F. Y. Edgeworth, Trans. Cambridge Phil. Soc. 20 (1905) 36.

[26] S. Hegyi and T. Csörgő, Proc. Budapest Workshop on Relativistic Heavy Ion Collisions, eds. T. Csörgő et al. (KFKI-1993-11/A, Budapest, 1991) p.47; T. Csörgő, Proc. Cracow Workshop on Multiparticle Production, eds. A. Białas et al. (World Scientific, Singapore, 1994) p.175; T. Csörgő, S. Hegyi, Phys. Lett. B489 (2000) 15.

[27] LEP design report volume I, CERN-LEP/TH/83-29 (1983);LEP design report volume II, CERN-LEP 84-01 (1984); LEP design report volume III, CERN-AC 96-01.

[28] L3 Collaboration, B. Adeva et al., Nucl. Instr. Meth. A323 (1992) 109.

[29] L3 Collaboration, A. Adam et al., Nucl. Instr. Meth. A383 (1996) 342.

[30] L3 SMD Collaboration, M. Acciarri et al., Nucl. Instrum. Meth. A351 (1994) 300.

[31] L3 SMD Collaboration, M. Acciarri et al., Nucl. Instrum. Meth. A360 (1995) 103.

[32] L3 Collaboration, M. Adriani et al., Nucl. Instrum. Meth. A302 (1991) 53.

[33] R. Brun et al., CERN/DD/EE/84-1 (Revised) 1987.

[34] H. Fesefeldt, RWTH Aachen Report PITHA 85/02 (1985).

[35] L3 Collaboration, M. Acciarri et al., Phys. Lett. B411 (1997) 373.

[36] A. Dominguez, Ph.D. thesis, University of California at San Diego (1998).

[37] R. E. Kalman, J. Basic. Eng. 83 (1961) 95.

[38] V. Innocente, M. Maire and E. Nagy, CERN Program Library W5013-E, 1991.

[39] JADE Collaboration, W. Bartel et al., Z. Phys. C 33 (1986) 23.

[40] JADE Collaboration, S. Bethke et al., Phys. Lett. B 213 (1988) 235.

[41] S. Catani et al., Phys. Lett. B 269 (1991) 432.

[42] S. Bethke et al., Nucl. Phys. B 370 (1992) 310.

[43] J. W. Gary, Phys. Rev. D49 (1994) 4503.

[44] A. H. Mueller, Nucl. Phys. B213 (1983) 85. 
[45] Yu. L. Dokshitzer et al., Rev. Mod. Phys. 60 (1988) 373.

[46] K. Konishi, A. Ukawa and G. Veneziano, Nucl. Phys. B157 (1979) 45.

[47] R. Odorico, Nucl. Phys. B172 (1980) 157.

[48] G. C. Fox and S. Wolfram, Nucl. Phys. B168 (1980) 285.

[49] T. D. Gottschalk, Nucl. Phys. B214 (1983) 201.

[50] J. Kalinowski, K. Konishi and T. R. Taylor, Nucl. Phys. B181 (1981) 221.

[51] J. F. Gunion and J. Kalinowski, Phys. Rev. D29 (1984) 1545.

[52] J. F. Gunion, J. Kalinowski and L. Szymanowski, Phys. Rev. D32 (1985) 2303.

[53] R. D. Field and R. P. Feynman, Nucl. Phys. B136 (1978) 1.

[54] B. Andersson et al., Phys. Rep. 97 (1983) 31.

[55] X. Artru, Phys. Rep. 97 (1983) 147.

[56] R. D. Field and S. Wolfram, Nucl. Phys. B213 (1983) 65.

[57] W. Bartel et al., JADE Collaboration, Phys. Lett. B101 (1981) 129.

[58] W. Bartel et al., JADE Collaboration, Z. Phys. C21 (1983) 37.

[59] T. Sjöstrand, Comput. Phys. Commun. 82 (1994) 74.

[60] T. Sjöstrand, Comput. Phys. Commun. 135 (2001) 135.

[61] G. Marchesini et al., Comput. Phys. Commun. 67 (1992) 465.

[62] G. Corcella et al., J. High Energy Phys. 01 (2001) 10.

[63] L. Lönnblad, Comput. Phys. Commun. 71 (1992) 15.

[64] C. Peterson et al., Phys. Rev. D27 (1983) 105.

[65] B. Andersson, G. Gustafson and B. Söderberg, Z. Phys. C20 (1983) 317.

[66] P. M, Stevenson, Phys. Rev. D23 (1981) 2916.

[67] S. Jadach and K. Zalewski, Acta. Phys. Pol. B28 (1997) 1363.

[68] K. Fiałkowski and R. Wit, Acta Phys. Pol. B28 (1997) 2039.

[69] K. Fiałkowski and R. Wit, Eur. Phys. J. C2 (1998) 691.

[70] K. Fiałkowski, R. Wit and J. Wosiek, Phys. Rep. D58 (1998) 09413.

[71] B. Andersson and W. Hofmann, Phys. Lett. B169 (1986) 364. 
[72] L. Lönnblad and T. Sjöstrand, Eur. Phys. J. C2 (1998) 165.

[73] F. James et al., Comput. Phys. Commun. 10 (1975) 343.

[74] P. Achard et al., Phys. Rep. 399 (2004) 71.

[75] OPAL Collaboration, G. Alexander et al., Z. Phys. C 72 (1996) 389.

[76] P. Achard et al., Phys. Rep. 399 (2004) 71. 


\section{Summary}

Bose-Einstein Correlations (BEC) of identical bosons can be used for the femtoscopy of the production properties of bosons in high energy particle collisions. This quantum mechanical BEC effect is a direct consequence of the symmetrization of the wave function of a boson system and is frequently used on photons in Astophysics to measure the angular size and other properties of distant stars. In particle collisions, the effect can be observed experimentally as an enhancement of the production of identical bosons with small four-momentum difference $Q$ relative to a production that would occur in a world without Bose-Einstein statistics.

In this thesis, BEC are studied between identical pions produced in electron-positron collisions at a center-of-mass energy of $91 \mathrm{GeV}$ in the LEP $\mathrm{e}^{+} \mathrm{e}^{-}$Collider of CERN, near Geneva. The final-state particles of these collisions are detected in the detector of the L3 experiment, which is positioned at one of the four intersections of LEP.

According to the present picture of boson production in electron-positron collisions, these two colliding particles annihilate to first produce a pair of a quark, $q$, and an antiquark, $\overline{\mathrm{q}}$, having the center-of-mass energy of the original electron-positron pair. The $q$ and $\bar{q}$ try to separate from each other but are held back by the strong color force acting between them. In the simplest case, this string-like field breaks into a number of further $q \bar{q}$ pairs which finally combine into particles. These particles are observed in the detector in the form of two jets of particles moving in opposite directions.

However, in the quantum chromodynamic field spanned between a $q$ and a $\bar{q}$, field quanta, so-called gluons, g, can be radiated. Contrary to photons in the case of quantum electrodynamics, these gluons themselves carry color charge and therefore act themselves as sources of the color field. In the next to simplest case, therefore, two string-like fields are spanned, one between the radiated gluon and the quark and one between that gluon and the antiquark. This leads to the production of three particle jets flying apart in different directions, but with the gluon jet containing two color string ends lying close to each other, both in momentum and configuration space.

The motivation for our investigation is the question whether bosons originating from different but overlapping strings or string pieces show BEC, i.e., whether so-called inter-string BEC exist between ovelapping strings or string pieces.

Statistics tells us that in the case of an increasing number of overlapping sources, the correlation strength $\lambda$ will decrease if these sources are independent. On the other hand, the source radius $R$ is expected to increase when the sources are dependent, since the average distance between two bosons is larger in this case. In particular, one would expect 
1. If there are no inter-string BEC but the two strings overlap:

$$
\lambda_{2 \text {-string }}<\lambda_{1 \text {-string }} \quad R_{2 \text {-string }} \approx R_{1 \text {-string }}
$$

2. If there are inter-string BEC and overlap between the two strings:

$$
\lambda_{2 \text {-string }} \approx \lambda_{1 \text {-string }} \quad R_{2 \text {-string }} \gtrsim R_{1 \text {-string }}
$$

3. If there are no BEC between two non-overlapping sources:

$$
\lambda_{2} \approx \lambda_{1} \quad R_{2} \approx R_{1}
$$

4. If there are BEC between two non-overlapping sources:

$$
\lambda_{2} \approx \lambda_{1} \quad R_{2}>R_{1}
$$

In this thesis, we have searched for differences in BEC between event configurations involving two color strings such as gluon jets and those involving single strings such as quark jets. Comparisons have been made of two-jet and three-jet events, of quark and gluon jets, and of pairs of identical pions originating from the same and from different sides of a gluon jet.

The strength parameter $\lambda$ and the size parameter $R$ of the Bose-Einstein correlation function $R_{2}$ are determined and compared for various configurations. The comparison of two-jet and threejet events is performed for various values of jet resolution and in different multiplicity intervals. Quark and gluon jets are compared for various energy ranges and in various windows of particle momentum. No significant difference is observed between two-jet and three-jet events, or between quark and gluon jets.

Furthermore, $R_{2}$ is compared for pions originating from the same side and from different sides of the gluon jet. Studies are performed in the gluon jet of various three-jet topologies (so-called Yq events, Mercedes events and Yg events), and in different particle momentum windows, both for light-quark and heavy-quark events. Again, no obvious difference is found between the same-side and the different-side pairs.

Referring back to our original motivation quoted above, at the given level of statistical significance, our results support our options (2) of the presence of BEC in pairs of pions originating from two different sources when these two sources are overlapping, or (3) of the lack of any overlap at all. Further analysis will be needed to distinguish between these two remaining alternatives. 


\section{Samenvatting}

Het verschijnsel van Bose-Einstein correlaties (BEC) tussen identieke bosonen kan gebruikt worden voor "femtoscopie" van de eigenschappen van bosonproductie bij botsingen van deeltjes met hoge energie. Het quantummechanische BEC effect is een directe consequentie van de symmetrie van de golffuncties van het bosonsysteem. In de astronomie wordt BEC tussen fotonen gebruikt om eigenschappen, zoals de grootte, van verafgelegen sterren te bepalen. Het BEC effect zorgt bij botsingen tussen hoog energetische deeltjes, voor een relatieve toename van de productie van bosonen met een klein verschil in vier-momentum $Q$. Dit is dan een toename ten opzichte van de productie die zou bestaan in een wereld waarin Bose-Einstein statistiek niet bestaat.

In dit proefschrift zijn BEC tussen identieke pionen bestudeerd. Deze pionen zijn ontstaan uit botsingen tussen elektronen en positronen in de LEP versneller van CERN bij Genève. Deze versneller versnelt de deeltjes tot een energie van $45.5 \mathrm{GeV}$. De elektronen en positronen botsen op vier plaatsen. In een van deze locaties staat de detector van het L3 experiment. De data beschreven in dit proefschrift zijn hieruit afkomstig.

Volgens het huidige model van boson productie bij elektron-positron botsingen, annihileren het elektron en het positron, en worden vervolgens een quark $\mathrm{q}$ en een antiquark $\overline{\mathrm{q}}$ in een paar gemaakt. Dit paar heeft dezelfde energie als het oorspronkelijke elektron-positron paar. Het quark en het antiquark bewegen uit elkaar, maar dit wordt tegengewerkt door de sterke (kleur) kracht. Het snaarachtige kleurenveld breekt in het meest eenvoudige geval in meerdere q $\bar{q}$ paren die uiteindelijk deeltjes gaan vormen. Deze deeltjes worden in de detector geobserveerd als twee jets (gecollimeerde stroom van deeltjes) die in tegengestelde richting bewegen.

Daarnaast kunnen in het quantum-chromodynamisch veld opgespannen tussen een $q$ en een $\bar{q}$ veld quanta, gluonen genoemd, uitgestraald worden. In tegenstelling tot fotonen, die de veld quanta zijn van het elektrodynamische veld, dragen gluonen wel (kleur) lading. De gluonen zijn dus ook bronnen van het kleurenveld. Net iets minder eenvoudig dan de 2-jet situatie is het geval waarin er twee snaarachtige kleurenvelden worden opgespannen, namelijk een tussen het uitgestraalde gluon en het quark, en een tussen dit zelfde gluon en het antiquark. Dit leidt tot het ontstaan van drie jets van deeltjes die in verschillende richtingen vliegen. De gluonjet bevat twee uiteinden van de kleurensnaren die zowel in configuratie als momentum ruimte dicht bij elkaar liggen.

Ons onderzoek wordt gemotiveerd door de vraag of bosonen die van verschillende, maar overlappende, snaren of stukken hiervan afkomstig zijn BEC laten zien. Met andere woorden, of er inter-snaar BEC bestaat tussen overlappende snaren of snaarfragmenten.

Het is op statistische gronden te verwachten dat de kracht van de correlatie $\lambda$ af zal nemen indien er steeds meer onafhankelijke maar overlappende bronnen aanwezig zijn. Aan de andere 
kant is te verwachten dat de straal van de bron $R$ toeneemt als de bronnen wel afhankelijk zijn. De gemiddelde afstand tussen twee bosonen is in dit geval immers groter. Het is mogelijk dat de bronnen wel of niet overlappen, en dat er tussen deze bronnen wel of geen BEC bestaat. In deze gevallen kan men verwachten dat:

1. In het geval dat er geen inter-snaar BEC bestaat, en de twee snaren overlappen:

$$
\lambda_{2 \text {-string }}<\lambda_{1 \text {-string }} \quad R_{2 \text {-string }} \approx R_{1 \text {-string }}
$$

2. In het geval dat inter-snaar BEC bestaat, en de twee snaren overlappen:

$$
\lambda_{2 \text {-string }} \approx \lambda_{1 \text {-string }} \quad R_{2 \text {-string }} \gtrsim R_{1 \text {-string }}
$$

3. In het geval dat er geen BEC is tussen twee niet-overlappende bronnen:

$$
\lambda_{2} \approx \lambda_{1} \quad R_{2} \approx R_{1}
$$

4. In het geval dat er BEC bestaat tussen twee niet overlappende bronnen:

$$
\lambda_{2} \approx \lambda_{1} \quad R_{2}>R_{1}
$$

In dit proefschrift hebben we de verschillen in BEC bestudeerd tussen verschillende types gebeurtenissen. We hebben gekeken naar de verschillen tussen gebeurtenissen waarbij twee snaarachtige kleurvelden opgespannen worden, en er gluonjets zijn, en gebeurtenissen waarbij een enkel veld opgespannen wordt en er alleen quarkjets zijn. De gebeurtenissen met twee en drie jets zijn met elkaar vergeleken voor quark- en gluonjets. Ook zijn paren van identieke pionen afkomstig uit dezelfde en verschillende stukken van de gluonjet vergeleken.

De kracht $\lambda$ en de uitgestrektheid $R$ van de Bose-Einstein correlatie functie $R_{2}$ zijn bepaald voor de verschillende gebeurtenissen. De vergelijking van botsingen waaruit twee en drie jets ontstaan is gedaan voor verschillende waarden van de zogenaamde jet-resolution, als functie van het aantal deeltjes. Quark- en gluonjets zijn vergeleken voor verschillende energieën, en in verschillende gebieden van de impuls van de deeltjes. Er is geen significant verschil gemeten tussen gebeurtenissen met twee jets en met drie jets. Ook is er geen significant verschil tussen quark- en gluonjets.

Daarnaast is $R_{2}$ vergeleken voor paren van pionen afkomstig uit dezelfde stukken en verschillende stukken van de gluonjet. Dit is bestudeerd in gluon jets afkomstig uit gebeurtenissen met drie jets in verschillende topologieën (genaamd Yq, Mercedes en Yg topologieën). Ook hier zijn verschillende gebieden van de deeltjes impuls bestudeerd voor gebeurtenissen waarbij zware en lichte quarks zijn gemaakt. Er is geen verschil gevonden tussen paren van pionen afkomstig uit dezelfde stukken en verschillende stukken van de gluonjet.

Terug komend op de motivatie van dit proefschrift, zoals hiervoor is beschreven. Onze resultaten zijn statistisch in overeenstemming met optie (2), waarbij BEC aanwezig is in paren van pionen die uit twee verschillende bronnen afkomstig zijn als deze bronnen overlappen, en met (3) waarbij er geen sprake is van overlap. Nadere analyse is nodig om onderscheid te maken tussen de overgebleven alternatieven. 


\section{Acknowledgements}

This thesis would not have been possible without the help, guidance and support of a lot of people. I will try to mention the people who have influenced me a lot during all these years' experience in the Netherlands.

First of all, I would like to show my deep gratitude to Prof. Dr. Wolfram Kittel, who offered this $\mathrm{PhD}$ position to me and this opportunity changed my life and career at the same time. I want to thank him for his trust and patient guidance on this complicated topic of Bose-Einstein correlations. I am deeply influenced by his way of thinking as a scholar and a mentor. He is not only a professor to discuss on physics, but also he is a friend. I remember the table tennis games played with him at the beginning of my $\mathrm{PhD}$.

Special thanks to my supervisor Dr. Wes Metzger. His daily guidance and critical remarks on my research greatly influenced the quality of the results. His continuous encouragement and great suggestions helped me a lot to recover from the frustrations. The discussions with him besides physics widened my mind and the different thought on some issues made me realize "I am never too old to learn". I am grateful to him that he was always ready to help me.

I would like to thank Prof. Dr. Sijbrand de Jong for his kind help in my study and in the "living-abroad" related issues.

I also like to thank the whole L3 Collaboration for the successful experiment and valuable data which are the basis of my thesis. I thank the L3 QCD analysis group for useful discussions during the meetings. Special thanks to Luca Malgeri and Arno Straessner for help on the analysis tools. Thanks to Šárka Todorova-Nová for useful discussions.

I enjoyed a lot being a member of the Experimental High Energy Physics Department because it was like a big family to me. I like to thank our secretaries Annelies Oosterhof-Meij, Marjo van Wees-Mobertz, Gemma Kopper-Janssen, Hanneke Vos-van der Lugt who made my life much easier in this country. Special thanks to Annelies Oosterhof-Meij for being my "second mother" here in the Netherlands. In particular, I would like to thank Peter Klok for being a great friend and always opening his arms to me. Thanks to Bram Wijngaarden, Lucian Ancu, Jorn van Dalen, Pieter Houben, Eric Jansen, Folkert Koetsveld and Martin van den Akker for their kindness. Special thanks to Miruna Anăstǎsoaie, Tamás Novák, Miran Djordjevic, Gustavo Ordonez for sharing the same office and daily feelings. I hope our friendship will continue. I thank Frans Rohde and Wim Janssen for support on computer issues. I thank Charles Timmermans for sharing the optimistic way of life all the time. Thanks to Susanne Kittel-Haböck for encouraging me with the words "when one door is closed, there is always another door open to you". I thank the friends from the Chinese community in Nijmegen. Special thanks to Ruifen Huo, Weibo Zhang and Xiuming 
Wu. Our group trips in Europe left me a lot of sweet memories. I also thank the members of the weekend badminton club in Nijmegen. The endless competition kept me energetic and my weekend life colorful.

Working at ASML gives me another chance to make new friends. I thank Florin Mezei, Paul Desmedt, Xu Yuan, Hannah Wei, Shuang Mu and Sicong Liu for their friendship. They make my life in Eindhoven more enjoyable.

I am infinitely grateful to my parents Chaozheng Wang and Huaju Guo, who gave me life and the chance to have a good education. Their deep love and unconditional support are the source of my courage. Thank you, my dearest parents, thank you and I love you!

And now, my beloved husband, Aleh Paskrakou. Meeting and marrying you changed my life totally and made it more meaningful. No words can express my feelings and how important you are to me. You are the biggest blessings to my life. 


\section{Curriculum Vitae}

The author of this thesis was born on 26th of January 1979 in Nanzhang (Hubei province, China). In 2000, she graduated with her Bachelor degree of Science from the Physics Department of Central China Normal University in Wuhan. From 2000 to 2002, she was a graduate student in the Institute of Particle Physics (IOPP) of Central China Normal University under the supervision of Prof. Liu Lianshou. In 2002, she received her Master's degree of Science, with the thesis "Model Study on Non-thermal Phase Transition and Antiproton to Proton Ratio at RHIC Energies". From August 2002, she started her PhD research as a junior researcher in the Experimental High Energy Physics Department of Radboud University Nijmegen (former Katholieke Universiteit Nijmegen) under the supervision of Prof. Dr. E.W. Kittel and Dr. W.J. Metzger, with the topic "Inter-string Bose-Einstein Correlations in Hadronic Z Decays using the L3 Detector at LEP".

During her study, she took part in the joint Belgian-Dutch-German graduate school on particle physics in 2002 (at Nijmegen) and 2003 (at Bonn). She attended the international summer school on particle and nuclear astrophysics "NIJMEGEN03" at Nijmegen in 2003. She presented a talk on part of the results of this thesis at the Dutch Physical Society (NNV) annual meeting for Nuclear and High-Energy physics in 2004. She participated in the international conference XXXV International Symposium on Multiparticle Dynamics at Kroměriž in the Czech Republic in 2005. She presented a poster at Lepton Photon 2005 at Uppsala in Sweden. She gave talks at the Workshop on Particle Correlations and Femtoscopy at Kroměřǐ in the Czech Republic and at the $5^{\text {th }}$ Budapest Winter School on Heavy Ion Physics at Budapest in Hungary in 2005. She acted as the tutorial teacher in the courses $\mathrm{C}$ programming and Modern Dynamical Physics.

Since November 2006, she has been working as a design engineer on nanochips at ASML in the Netherlands. 